$$
\text { PNNL--10924 }
$$

\title{
Pacific Northwest National Laboratory Institutional Plan FY 1996-2001
}

January 1996

Prepared for the

U.S. Department of Energy

under Contract

DE-AC06-76RLO 1830

Pacific Northwest National Laboratory

Richland, Washington 99352 


\section{Disclaimer}

This report was prepared as an account of work sponsored by an agency of the United States Government. Neither the United States Government nor any agency thereof, nor Battelle Memorial Institute, nor any of their employees, makes any warranty, expressed or implied, or assumes any legal liability or responsibility for the accuracy, completeness, or usefulness of any information, apparatus, product, or process disclosed, or represents that its use would not infringe privately owned rights. Reference herein to any specific commercial product, process, or service by trade name, trademark, manufacturer, or otherwise does not necessarily constitute or imply its endorsement, recommendation, or favoring by the United States Government or any agency thereof, or Battelle Memorial Institute. The views and opinions of authors expressed herein do not necessarily state or reflect those of the United States Government or any agency thereof.

\section{Pacific Northwest National Laboratory}

Operated for the

U.S. Department of Energy

by Battelle 


\section{Contents}

1 Director's Statement .............................................................................................................................................. 1

2 Laboratory Mission and Core Competencies .................................................................................................. 3

Mission .............................................................................................................................................. 3

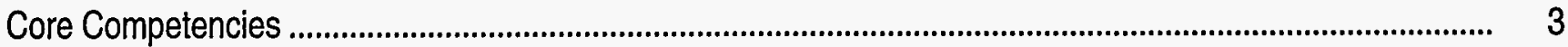

3 Laboratory Strategic Plan ............................................................................................................................ 5

Primary Planning Assumptions ................................................................................................................... 5

Strategic Objectives: Laboratory Support to the Department of Energy Strategic Plan ..................................... 6

Fundamental Strategies ............................................................................................................................... 9

4 Laboratory Initiatives ..................................................................................................................................... 11

Molecular Sciences Research .......................................................................................................... 12

Microbial Biotechnology ....................................................................................................................... 16

Global Environmental Change ..................................................................................................................... 18

Complex Modeling of Physical Systems ..................................................................................................... 20

Advanced Processing Technology ....................................................................................................... 22

Energy Technology Development............................................................................................................ 25

Medical Technologies and Systems ...................................................................................................... 27

5 Core Business Areas ........................................................................................................................................... 32

Science and Technology ................................................................................................................ 32

Environmental Molecular Sciences Laboratory ................................................................................... 32

Office of Energy Research ................................................................................................................. 40

Science, Mathematics, Engineering, and Technology Education ........................................................... 54

Environmental Quality .................................................................................................................................. 61

Office of Environmental Management ......................................................................................................... 62

Office of Environmental Safety and Health ........................................................................................... 76

Energy Resources .......................................................................................................................... 81

Office of Energy Efficiency and Renewable Energy ........................................................................... 81

Bonneville Power Administration .............................................................................................................. 87

Office of Civilian Radioactive Waste Management .................................................................................... 89

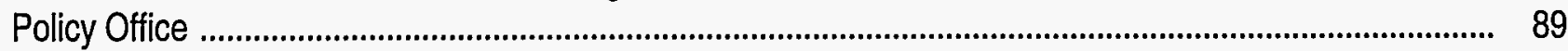

Office of Nuclear Energy ...................................................................................................................... 90

Office of Fossil Energy .......................................................................................................................... 91

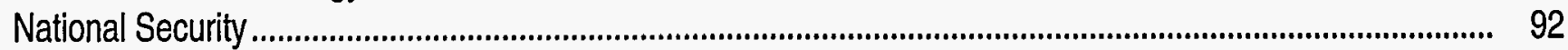

Office of Defense Programs .................................................................................................................. 93

Office of Nonproliferation and National Security ......................................................................................... 93

Economic Productivity ....................................................................................................................... 96

Enhanced Awareness of Industrial Needs Throughout the Laboratory ...................................................... 96

Enhanced Industrial Awareness of the Laboratory ....................................................................................... 97

Providing Technical Assistance and Collaborating with U.S. Industry ..................................................... 99

Commercialization of Intellectual Property ................................................................................................ 102 
Laboratory Directed Research and Development Program .......................................................................... 104

Program Administration and Management ............................................................................................. 104

Scientific and Technical Investment Areas ............................................................................................. 105

Selected Highlights of LDRD Projects ...................................................................................................... 105

Work for Other DOE Sites ........................................................................................................................... 106

Work for Others ......................................................................................................................................... 106

Department of Defense .................................................................................................................... 106

Nuclear Regulatory Commission .............................................................................................................. 108

Environmental Protection Agency ......................................................................................................... 110

Health and Human Services.......................................................................................................... 111

National Aeronautics and Space Administration ................................................................................... 111

Federal Emergency Management Agency ........................................................................................ 112

Other Federal Agencies and Nonfederal ................................................................................................. 112

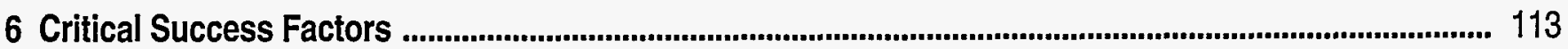

Human Resources .......................................................................................................................... 113

Laboratory Personnel ........................................................................................................................ 113

Affirmative Action and Equal Employment Opportunity ......................................................................... 115

Environment, Safety, and Health Management ............................................................................................... 118

ES\&H Goals and Objectives ....................................................................................................... 118

Current Conditions ............................................................................................................................ 119

ES\&H Policies, Organization, and Management .................................................................................. 119

ES\&H Plans and Initiatives ............................................................................................................ 120

Energy Research Funded ES\&H Improvement Items ............................................................................ 122

Environmental Restoration and Waste Management Activities ................................................................... 123

Hanford Site-Wide Funded ES\&H ................................................................................................... 124

Information Resource Management .................................................................................................. 125

Information Technology Vision ................................................................................................... 125

Environment and Accomplishments .............................................................................................. 126

Major Goals and Near-Term Strategies .................................................................................................. 130

Major Items of Equipment ................................................................................................................... 132

Site and Facilities.............................................................................................................................. 132

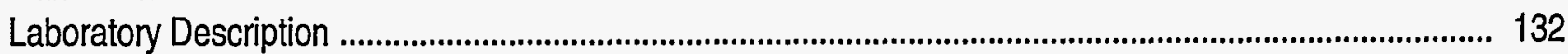

Consolidated Information Center ............................................................................................................ 141

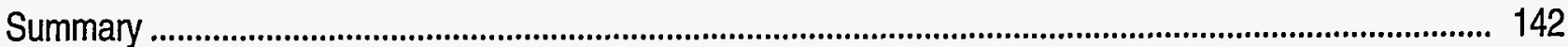

Communication and Trust................................................................................................................... 142

7 Resource Projections .......................................................................................................................................... 143

Subcontracting and Procurement ......................................................................................................... 156

Small and Disadvantaged Business Procurement .................................................................................. 156

8 Acronyms and Abbreviations ......................................................................................................................... 158 
1995 was a very challenging year for all of us. However, I am convinced that Pacific Northwest National Laboratory is now better positioned than ever to contribute impactful science and technology to the nation. We can be confident of a bright future, in which we can deliver relevant, technologybased value for our customers and create new opportunities for our staff.

This bright future is the natural consequence of our history. From the 1965 restructuring of the Hanford Site through the early 1980 s we built a solid record of excellence in engineering and technology development. We were able to meet complex challenges associated with the development of the domestic nuclear power industry, including the nuclear fuel cycle, engineering design and safety analysis, and materials issues. We also built capabilities that have led to our continuing role in radiation health effects and protection research and we made worthwhile contributions in the life, materials, and environmental sciences. Our "distinctive signature," though, was excellence in technology development. If you wanted to get something built and working, this Laboratory was the place to come.

In 1986 we were designated an Energy Research laboratory, bringing us into a family of laboratories with an outstanding scientific tradition. Then-Director Bill Wiley and our leadership team saw that to succeed we needed broader, more impactful basic research capabilities. We've met this daunting challenge with the strong encouragement and support of the U.S. Department of Energy's Office of Energy Research. We now have scientific capabilities fully on par with our tradition of engineering excellence. We're nearing completion of the Environmental Molecular Sciences Laboratory, the first major DOE scientific user facility in the Northwest.
We have built leading-edge programs in theoretical and experimental chemistry and greatly strengthened our research capabilities in materials science, computing and information science, and selected aspects of molecular biology. We've also expanded our participation in DOE's environmental science programs, and I'm particularly proud of our contributions to the Atmospheric Radiation Measurement program and the Subsurface Science Program.

The last ten years also saw rapid growth, with our staff increasing from about 2,900 at the beginning of 1985 to a peak of a little more than 4,600 in 1994 . This growth was caused by our ability to respond quickly to DOE's significant environmental challenges and our increasing role in DOE's science programs. Invention and deployment of a broad family of vitrification technologies, as just one example, showed that our applied research and engineering capabilities had not lost their edge. This decade also demonstrated our flexibility, as our engineering focus shifted from nuclear to environmental technology. This ability to adapt rapidly is a hallmark of this Laboratory that we will surely depend on in the years to come.

As a result of our history, then, we entered 1995 with a powerful and rapidly maturing combination of basic and applied science and engineering capabilities. As you all know, however, 1995 has been a year of transition for the entire DOE laboratory system. The role and value of the national laboratories have been vigorously debated. Budget constraints and changing national priorities have led to reduced funding in many areas and focused attention on the issue of productivity. There is still a very real possibility that some major federal

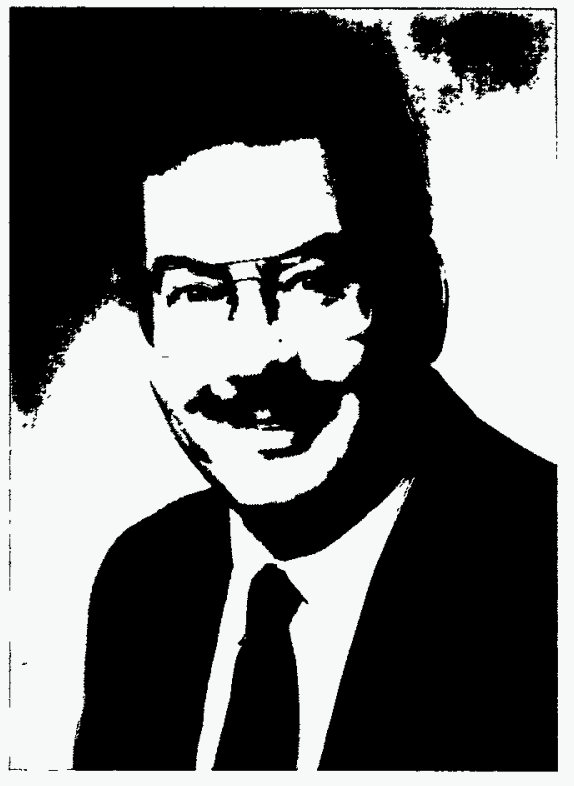

Dr. William J. Madia, Director, Pacitic Northwest National Laboratory

laboratories may be closed in the next few years.

I look back on this turbulent year with mixed emotions. We faced the difficult and painful task of saying goodbye to many of our colleagues as we reduced the size of the Laboratory in line with declining research funding and as we adjusted our staffing mix. Many of those who left had been with us for substantial parts of their careers, and we all had good friends among them. They have made significant contributions to our Laboratory, and they have both our thanks and our best wishes. They will always be part of our heritage.

I believe we'll also look back on 1995 as the year in which we built the foundation for renewed growth and new opportunities. We initiated a major change program designed to ensure that our Laboratory was both relevant to national needs and highly productive, and I'm pleased to say that we've made dramatic progress in both regards. 
We completed our Achieving the Competitive Edge program in October, and with the support of the DOE Richland Operations Office, we are continuing to implement the impactful recommendations of our breakthrough teams. Already advances like electronic time reporting and purchasing cards have reduced costs and improved service to our research projects. We've streamlined our organization and drastically reduced senior management costs. We can all take pride in the fact that the Secretary of Energy has recognized our Laboratory as the productivity pacesetter in the DOE system, and I want to thank the many staff who have shown leadership through their service in the ACE program.

We've also established a much clearer mission focus. We've defined the delivery of environmental science and technology in the service of our nation as our core mission, although not our only mission. This core mission ties us closely to DOE's environmental mission, and to the nation's long-term need for technologies that minimize waste and prevent pollution. It also reflects our substantial role in the Department's science programs, including environmental sciences and related aspects of the physical and life sciences, and speaks to our leading-edge interdisciplinary work in global change. In the other missions we serve, too, we are closely tied to enduring national needs. In national security we concentrate on arms control and nonproliferation, in energy on transmission and distribution and energy efficiency issues, and in health on the molecular-level processes underlying human health risk. Overall, we have implemented a holistic and synergistic mission framework.

Over the last year we've also greatly improved our operational performance. During 1994 our customer expressed significant concerns about our facilities operations and about our Environment, Safety, and Health program. In response, and working closely with DOE-RL, we implemented the Operations Improvement Program and thoroughly revamped our ES\&H organization. I'm delighted to say that these efforts have paid off in significantly improved performance and in muchimproved DOE evaluations in this area. I want to thank the many staff whose thoughtful and dedicated efforts have yielded this improvement.

The announcement of our new namePacific Northwest National Laboratory-by the Director of Energy Research, Dr. Martha Krebs, was a real highlight of our Onsite Review this fall. This change confirms our success in building the leading-edge scientific capabilities that make us a full-fledged member of the Energy Research family. It also reflects DOE's long-term commitment to the future of our Laboratory.

Let me now turn to that future. Over the next few years, we will apply our substantial resources and energy to focused growth and scientific excellence with the same intensity we brought to our productivity challenge. Delivery of high-value science and technology in response to critical national needs is the key to the growth I believe necessary to fully realize our potential. As we are successful, that growth will take place through selective addition of technical staff, creation of new scientific facilities, and expanded partnerships with research universities and industry. Whatever the mechanisms, it will mean new opportunities for each of us to advance in our careers and contribute to our nation.

We must deliver the Environmental Molecular Sciences Laboratory as a state-of-the-art collaborative research facility. We must complete this complex construction project and its complement of first-of-a-kind scientific instrumentation. We must also build the scientific programs and collaborations that ensure that this major DOE investment has rapid and substantial impact on the Department's environmental mission.

We are also committed to providing the Department with technology that substantially advances the cleanup mission here at Hanford and across the DOE complex. Our success will be determined by our ability to deploy technology that reduces costs, speeds progress, or improves the effectiveness of the cleanup. And in each of our other mission areas as well, our first measure of performance is our ability to deliver impactful knowledge and technology that meets real needs.

We will continue to be challenged by rapid change. However, with that change comes new opportunity. Indeed we are really in the business of change - that's what science and technology is all about. The major issues confronting our nation must at least in part be solved through new science and technology. There will always be new opportunities for productive research institutions that deliver high-quality work that addresses pressing needs. At Pacific Northwest National Laboratory we have a solid foundation of engineering and scientific excellence and an outstanding staff. With the continuing relevance of our missions, and the dramatic improvements in productivity and operations we've made in the last year, we are ready for whatever change tomorrow may bring.

I am convinced that "our past is prologue" to a very bright future. We will be serving our nation with distinction for many years to come.

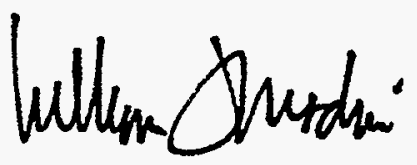




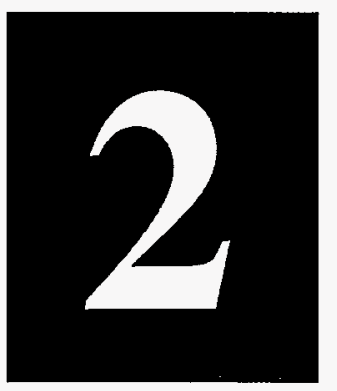

\section{Laboratory Mission and Core Competencies}

During the past 2 years, we have been carefully reexamining the mission and capability portfolio of the Pacific Northwest National Laboratory. We realized that in a time of limited resources we had to make careful choices both about what problems we would solve and the technical capabilities we would maintain. This reexamination was lent added impetus by our participation in the review process carried out by the Task Force on Alternative Futures for the Department of Energy laboratories and by the release of the Department of Energy's Strategic Plan.

We reached two conclusions while reviewing the Laboratory's portfolio of projects and technical capabilities:

- Fully two-thirds of the Laboratory's work is in environmental science, environmental technology, or both. Further, our projects in support of DOE's national security and energy missions draw heavily on capabilities we have developed in support of our environmental mission.

- The distinguishing feature of the Laboratory is the ability to bring multiple technical and support capabilities together to solve complex problems. Although we are proud of our strength in a number of technical disciplines, our core competencies must be more broadly defined.

On the basis of these considerations, we have sharpened both our mission and the definition of our core competencies.

\section{Mission}

Pacific Northwest National Laboratory's core mission is to deliver environmental science and technology in the service of the nation and humanity. Through basic research we create fundamental knowledge of natural, engineered, and social systems that is the

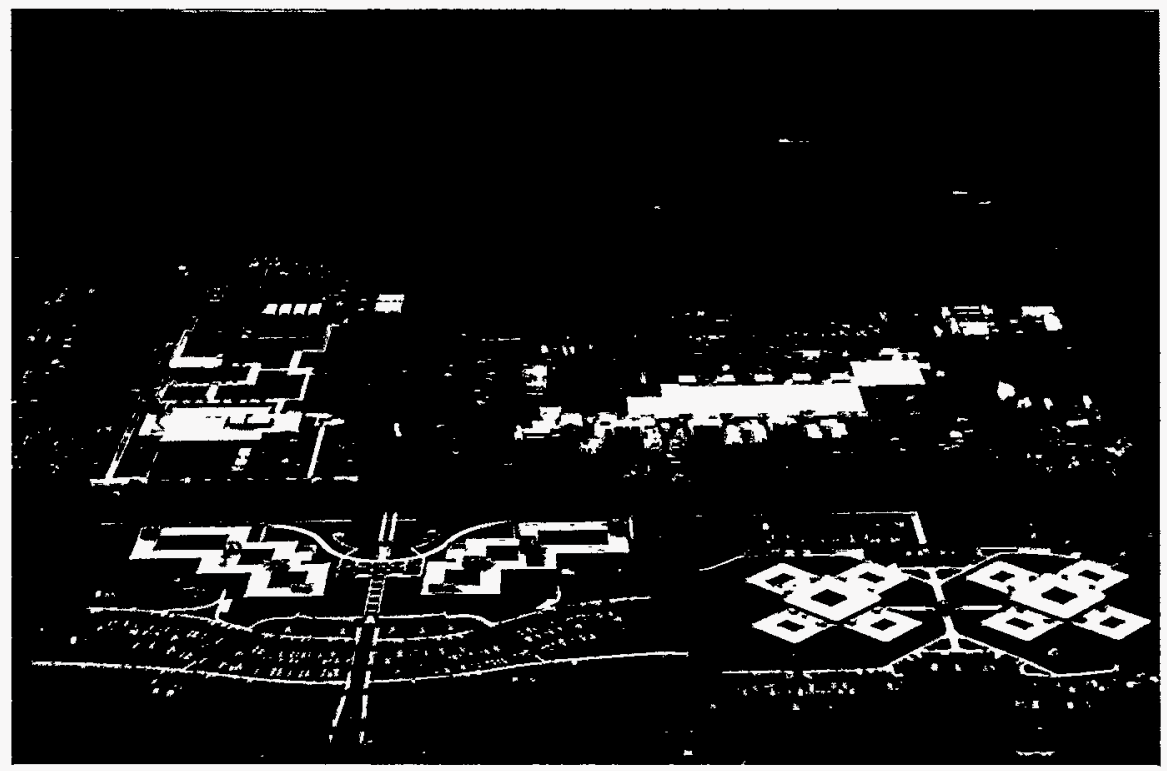

Pacific Northwest National Laboratory's Richland facilities

basis for both effective environmental technology and sound public policy. We solve legacy environmental problems by delivering technologies that remediate existing environmental hazards, we address today's environmental needs with technologies that prevent pollution and minimize waste, and we are laying the technical foundation for tomorrow's inherently clean energy and industrial processes. We also apply our capabilities to meet selected national security, energy, and human health needs; strengthen the U.S. economy; and support the education of future scientists and engineers.

\section{Core Competencies}

The concept of "core competencies" has become increasingly central to the management of the Laboratory over the last few years. We view core competencies as those few broad, integrated capabilities that allow us to deliver robust solutions to the most complex environmental challenges facing the Department of Energy and the nation. Pacific Northwest National Laboratory has developed and maintains the following three core competencies:

\section{- Integrated Environmental Research} encompasses the capabilities necessary to provide a whole-system approach to environmental issues, including sensing and measurement of environmental contaminants; characterization of physical processes in the earth's interior, ocean, and atmosphere; the transport and fate of contaminants in the environment; the interaction of biological or ecological systems with their physical environment and their response to contaminants in that environment; and the technical, economic, and regulatory analysis of policy alternatives.

- Process Science and Engineering encompasses the capabilities necessary to develop process technology for both environmental remediation 
and for pollution prevention and waste minimization, including advanced chemical separations, the mechanics of complex fluids, selected aspects of catalysis, selected aspects of materials science, and process design and testing.

- Energy Systems Development encompasses the capabilities necessary to design and efficiently operate environmentally benign energy generation, distribution, and utilization technologies, including automated diagnostics and controls, buildings science, power system engineering, and selected aspects of materials engineering and specialty component fabrication.

Each of these three core competencies is a synthesis of the technical and other capabilities necessary to deliver complete solutions to broad classes of problems. Our strategies for capability development are based on the health of these competencies and on our understanding of the future problems to which they will be applied.
To ensure that our core competencies remain on the leading edge, we also manage the underlying technical capabilities, which we define along more traditional disciplinary lines. Technical capability leaders maintain active internal networks to ensure a high level of internal and external communication, and prepare assessments of the capabilities and development plans which are designed to ensure continued excellence and relevance in each of the technical capabilities. 


\section{Laboratory Strategic Plan}

For the Department of Energy laboratories, this remains a time of rapid change, unprecedented in recent times. The nation's security needs have been redefined by both the end of the Cold War and the potential proliferation of nuclear and other weapons technologies. Domestic priorities are being rethought in light of economic and fiscal constraints. We face fierce competition from developed and developing nations as the economic predominance that we took for granted only a few years ago has eroded. Economic growth in the developed nations and population growth and the drive toward increased standards of living in the developing world are intensifying our environmental challenges. As a result of these and other changes, the social contract under which the nation's taxpayers have broadly supported scientific research, formed at the end of the Second World War and strengthened in the space era, is being reexamined.

In this time of change, the nation is reviewing its commitments to science and technology and reevaluating the public institutions charged with meeting those commitments. As with other federal laboratories, the mission, size, governance, and even existence of the Department of Energy laboratory system is in question. The Task Force on Alternative Futures for the Department of Energy National Laboratories, led by Robert Galvin, released its report in February 1995. This report recommended sweeping changes in governance and management practice. The Task Force also urged an increased focus on the national security, energy, and environmental missions, and on related basic research, and identified what it believed to be overcapacity in the laboratory system. Similar reviews of other major elements of the federal laboratory system are ongoing under the purview of the National Science and Technology Council. In a broader vein, the overall federal research and development structure is being rethought, both in Congress and in the Administration.

We believe that it is quite likely that there will be significant changes in the federal research and development infrastructure. Although the political process is to some extent unpredictable, we believe that there is a substantial likelihood that the overall size of the federal laboratory system will be reduced, and the premise for federal funding in each of our missions will be reexamined. How should we respond?

Our fundamental goal is to make the Pacific Northwest National Laboratory the most relevant and productive supplier of science and technology in those areas where we concentrate our efforts. By relevance, we mean that we will focus on the highest value needs of DOE and the nation. Our wôrk must be understood, valued, and supported by not only the scientific community and policymakers, but also by the public and its elected representatives. Our view of productivity encompasses the four key elements of quality, timeliness, cost-effectiveness, and stewardship. We must provide timely delivery of high-quality products at reasonable cost while exercising stewardship for our staff, intellectual capital, research equipment and facilities, community, and environment.

\section{Primary Planning Assumptions}

A set of broad assumptions underlie our planning. These assumptions, which frame our picture of the future world in which we must operate and in which our strategies will be tested, are as follows:
- A reduction in overall size of the federal laboratory system is inevitable, with a reduction in the work force of the Department of Energy laboratory system also highly likely. Closure of federal laboratories is possible.

- Resources of all types will be limited. The total national investment, public and private, in science and technology will grow slowly over the next few years. Federal investment will be sharply constrained for the foreseeable future by the ongoing attempt to balance the federal budget. As a result, there will be unprecedented technical and political competition for scarce investment resources.

- We will return to the original "Government-Owned, ContractorOperated" concept for laboratory management. The Department of Energy and its national laboratories are under fierce pressure to improve management practices and increase productivity. DOE's response to the report from the Galvin Task Force and the Department's overall effort to reduce costs will result in laboratory management having both greater independence of action and greater accountability for results.

- Federal research, development, and application investments will be strongly and explicitly tied to the most pressing technology and policy needs. An increased share of the available resources will be allocated to needs-driven research programs in areas such as health care, transportation, environmental remediation and protection, national security, and energy reliability. Even basic research investments will increasingly have to be justified in terms of expected near-term benefits in high priority areas. 


\section{Pacific Northwest National Laboratory's Strategic Objectives}

- We will deliver the Environmental Molecular Sciences Laboratory as a preeminent collaborative research facility, and in so doing bring the nation's best scientific talent to the Department's critical environmental challenges.

- We and our research partners will deliver knowledge required to make scientifically sound riskbased decisions and deliver technology that reduces the cost and improves the timeliness and effectiveness of environmental remediation at Hanford and across the entire DOE complex.

- We and our partners will develop and deploy technologies and strategies that increase the efficiency, improve the reliability, and reduce the adverse health and environmental impacts of energy systems.

- We and our partners will develop technologies that measurably help modernize America's manufacturing and process industries, thus creating new high-wage jobs and increasing U.S. export potential. In particular, we will make major contributions to the regional economy, and to the transition of the local economy to the post-Hanford era.

- We will develop arms control, nonproliferation, and intelligence technologies that enhance national security and reduce the danger from weapons of mass destruction.
- We are committed to building a tradition of interactive personal and institutional leadership, encompassing the entire staff of the Laboratory.

- We will manage our facilities and conduct our work in a manner that protects the environment and the health and safety of our staff and the public.

- We will be the benchmark case for management of national laboratories in terms of value of the work we do; the quality of the working environment; our organizational effectiveness; the stewardship of our human, physical, and intellectual assets; and our cost-effectiveness.
- The tension between economic development, or meeting human needs, and protection of our environment will be a primary driver for science and technology investments for the foreseeable future. Technology that allows economic growth while maintaining our environment is critical, both to enhance our and our neighbors' standard of living and to preserve our international competitive position.

- Although industrial research and development investments should show continuing modest growth, the well-documented trend in industrial investment toward short-term, product-related research will likely continue. Industry must, therefore, look primarily to government supported research in the universities and federal laboratories to provide the new knowledge from which breakthrough commercial technologies can arise.

- Advances in scientific instrumentation, computing and information technology, scientific knowledge, and engineering practice will be very rapid by past standards. Continual reinvestment in the knowledge and expertise of our staff and in our research equipment and supporting infrastructure will be required if we are to remain relevant, productive, and competitive for research funding.

- We will face substantial challenges in attracting, retaining, and motivating staff through at least the next several years as we deal with the combined impacts of funding uncertainties and work force restructuring.

\section{Strategic Objectives: Laboratory Support to the Department of Energy Strategic Plan}

In this section we present our highlevel or strategic objectives in the context of the Department of Energy's Strategic Plan, most recently released in 1994. The Department's Strategic Plan establishes goals in each of its core missions: Science and Technology, Environmental Quality, Energy Resources, and National Security. In addition, the plan articulates DOE's role in strengthening the nation's economy as a derivative outcome of success in these core missions. The plan also discusses a number of critical success factors that define the principles by which DOE conducts its work.

As stated in our mission, the Laboratory is concentrated on the environmental quality mission and the scientific research required to support that mission. We also support the energy resources and national security missions in areas where there exists an overlap between our core competencies, built upon past accomplishment and our accumulated skills, and the DOE's goals. In addition, we have set strategic objectives in several of the areas represented by DOE's critical success factors.

\section{Science and Technology}

The DOE Strategic Plan establishes five primary goals in Science and Technology. These goals include providing science and technology core competencies that enable DOE to achieve is missions, providing new insight into the nature of matter and energy, effectively constructing leading-edge user facilities, adding value to the U.S. economy through application of new technology, and 
enhancing science education. In keeping with our environmental mission, our science portfolio has two primary components. We are heavily engaged in the environmental sciences, including the investigation of the processes by which contaminants are transported and transformed in the environment and the impacts of those contaminants on ecological systems. We also maintain leading-edge programs in those aspects of the chemical, material, and life sciences that underlie creation of environmental technology, or that are required to understand the interaction between natural and biological or engineered systems. Through these programs we support four of DOE's five goals; we have no significant role in DOE's high energy and nuclear physics.

Our primary objective in support of DOE's Science and Technology mission is to deliver the Environmental Molecular Sciences Laboratory as a preeminent collaborative research facility, and in so doing, bring the nation's best scientific talent to the Department's critical environmental challenges. We intend for operation to begin in early FY 1998, at which point we will have completed construction of the conventional facility on schedule and on budget. We are committed to provide the maximum possible scientific capabilities in this Laboratory.

Over the next 5 years we intend to continue our emphasis on bringing new scientific knowledge to bear on DOE's environmental, energy, and national security missions. For example, the programs housed in the EMSL will be fully successful when the resulting knowledge has significant impact on DOE's environmental remediation programs at Hanford and across the complex, and more broadly, on the environmental management challenges facing the nation. Similarly, our energy programs increasingly reflect the application of basic scientific and engineering work, such as our microtechnology investments, to the creation of new, environmentally benign, pollution preventing technologies. Our nonproliferation and arms control programs must remain well-connected to our environmental science and technology base in areas such as radiation detection and chemical sensors.

We will continue our contributions to systemic reform of scientific and mathematics education in support of DOE's efforts to both provide a technically trained, diverse work force of the future and to ensure a technically literate citizenry. We will continue to concentrate on curricular reform, on regional outreach, and on strengthening our ties with historically black colleges and universities and other minority institutions. We intend to steadily increase the fraction of our research that is carried out in partnership with the university community, and to continue providing research participation opportunities to visiting students, teachers, and university faculty.

\section{Environmental Quality}

In Environmental Quality, we have strong leadership roles in the two principal science and technology goals asserted in the DOE Strategic Plan: reduction of the health and environmental risks associated with both legacy production sites and current operations, and development of environmental technology. We and our research partners will deliver knowledge required to make scientifically sound risk-based decisions and deliver technology that reduces the cost and improves the timeliness and effectiveness of environmental remediation at Hanford and across the entire DOE complex. We will support DOE in achieving a scientifically defensible, risk-based remediation strategy and schedule, and deliver technology that helps DOE . meet that schedule at affordable cost. To fulfill this intent, we have added a fundamental molecular sciences capability to our long-term strength in the environmental sciences. On the engineering side, we are strengthening our system engineering skills to complement our long-standing capability in the design of complex processing systems.

To fulfill the Department's strategic intent and to meet the expectations of the nation's taxpayers, the environmental remediation program requires intense effort over the next few years. In the long term, however, a strategy of pollution prevention and environmental protection must replace the strategy of waste management and environmental restoration. We must protect our environment through use of inherently clean processes rather than through costly post-pollution remediation. Pacific Northwest National Laboratory, in partnership with our sister laboratories and with U.S. industry, will be a national resource of environmentally acceptable technology and, again in partnership with our laboratory and university colleagues, will provide the underlying science required for future advances. Our principal focus areas, in line with the DOE Strategic Plan and as reflected in our current initiatives, are industrial process technology and commercial and residential energy end-use technologies.

\section{Energy Resources}

We believe that DOE's Energy Resources mission will be of increasing importance to the nation for the foreseeable future. The DOE Strategic Plan specifies four primary goals in this mission area: enhancing energy productivity; ensuring reliable, secure energy supplies; reducing the environmental impacts of energy production, delivery, and use; and promoting economic and regional equity through enhancing energy production, delivery, and usage systems. Our programs leverage our environmental science and technology capabilities to support the first three of these goals.

Our broad objective in the energy area can be stated in language similar to that used by DOE in the Strategic Plan. We and our partners will develop and deploy technologies and strategies that increase the efficiency, improve the reliability, and reduce the adverse health and environmental impacts of energy systems. Our ongoing analysis supports the three areas that emerged from our strategic planning effort of 4 years ago as timely and appropriate to our Laboratory. Accordingly, we will continue our focus on enhancing the resource efficiency and performance of existing energy assets (and most particularly 
transmission and distribution systems), and our work with government and the utility industry to develop a new generation of distributed energy systems. We will also continue our investment in the capabilities required to develop and deploy resource-efficient pollution prevention and waste minimization technologies that enhance the economic, energy-efficiency, and environmental performance of government and U.S. industry.

In executing these three thrusts, we intend to continue placing special emphasis on the Northwest region, including our long-time partnership with the Bonneville Power Administration and our engagement with regional and state energy policy and technology organizations. The development of effective partnerships around major Northwest energy issues, such as management of our watersheds to accommodate the often conflicting multiple objectives of power generation, habitat preservation, and supply for agricultural uses, is an important element of our strategy.

Finally, we are supporting the Department's efforts to help developing and rebuilding countries create a balanced, coordinated, and sustainable portfolio of energy sources; efficient or sustainable energy consumption practices; and a reliable energy infrastructure. Over the last 2 years, we have become increasingly engaged in Eastern Europe, the Far East, and Central and South America through the creation of independent Energy Efficiency Centers, and we expect to continue to play an active role. We believe that this outreach effort will be of increasing importance to DOE.

\section{National Security}

The DOE Strategic Plan establishes five primary national security goals. Of these, we significantly support DOE's nonproliferation and arms control goal. The rationale for our involvement is straightforward. The Laboratory's core technical capabilities and key technologies, developed through our environmental science and technology programs have direct application to critical nonproliferation and arms control challenges facing the
Department. In addition, and of particular current interest, our expertise in nuclear power and related technologies is highly relevant to current U.S. policy goals to reduce the danger associated with power producing reactors in the Former Soviet Union. We are not, and have no intention of becoming involved in maintenance of the DOE national security infrastructure, in weapons science and technology, or in weapons dismantlement.

Our primary objective is to leverage our environmental science and technology base to develop arms control, nonproliferation, and intelligence technologies that enhance national security and reduce the danger from weapons of mass destruction. We also pride ourselves on the ability to respond rapidly to urgent technology needs in our areas of expertise and plan to maintain that capability. Finally, in the last 2 years, we have assumed significant programmatic and technical responsibilities in DOE's effort to reduce the danger associated with power reactors in the Former Soviet Union. Over the next 5 years we will devote substantial staff and management resources to meeting those responsibilities.

\section{Economic Productivity}

As a derivative of the Department's core missions, the DOE Strategic PIan lays out a broad set of goals in the economic area, including helping industry make the transition to pollution prevention and waste minimization, becoming a more reliable partner for industry, speeding technology deployment, and developing "dualbenefit" technology that meets both DOE mission needs and is of commercial value to U.S. industry. In supporting these goals, we must keep in mind the strong commitment that DOE has made to local economic transition to the post-cleanup world, in which thousands of DOE-supported jobs will disappear and must be replaced by private-sector activities over the next two to three decades. Accordingly, we and our partners will develop technologies, primarily in the environmental area, that measurably help modernize America's manufacturing and process industries, thus creating new high-wage jobs and increasing U.S. export potential.

With regard to the Northwest region and the local economy, we believe that we have a critical role to play in supporting DOE's commitment to economic transition. This commitment is central to the Hanford Strategic Plan that has been released by the DOE Richland Operations Office, and must be met to ensure that completion of Hanford cleanup does not result in massive economic dislocation. Our intent is to ensure that a vital Pacific Northwest National Laboratory can serve as an economic anchor for the community and an integral part of the Northwest science and technology infrastructure. In addition, we are setting ourselves the objective of increasing the number of successful mid-Columbia companies created by spin-off from our Laboratory, producing products based on our technology, or otherwise catalyzed by our actions. Finally, through an expanded portfolio of outreach programs, we intend to be a productive and increasingly sought-after technology resource to local and regional small businesses.

\section{Critical Success Factors}

In addition to setting objectives in each of its missions or core business areas, the DOE Strategic Plan lays out a clear vision of how the Department will be managed. The plan establishes goals associated with each of four primary critical success factors: communications and trust; human resources; environment, safety and health; and management practices. Broadly speaking, these critical success factors and the associated goals represent a commitment by DOE to be "best in class" in meeting the performance expectations of the public.

We have established three high-level objectives in the areas covered by DOE's critical success factors that we believe are essential to the continued success and vitality of the laboratory. First, we recognize that the heart of the laboratory is made up not of facilities or equipment, but by our research and administrative staff. Accordingly, 
we are committed to building a tradition of interactive personal and institutional leadership, encompassing the entire staff of the Laboratory. We will recruit, develop, and retain a diverse staff recognized for scientific, intellectual, and personal leadership for the integrity of our research and business practices, and for our contributions to the community and the nation. This commitment takes on particular importance in light of the programmatic and staff reductions that we experienced in 1995.

Second, the Department's Strategic Plan communicates a strong and unambiguous commitment to operations to ensure the health and safety of our work force and the public and with respect for the environment. We will manage our facilities and conduct our work in a manner that protects the environment and the health and safety of our staff and the public. We have been moved to establish this commitment as one of our top level objectives by the recognition that, although our environment, safety, and health statistics are good as compared to industry standards, our operating practices may not yet fully conform to the standard of excellence expected by DOE and the public. We will evolve as rapidly as possible through implementation of a Laboratory-wide operations improvement program to an improved level of performance. In carrying out this effort, we are working with DOE to establish the appropriate standards for each aspect of our operations, and designing management systems to ensure that we cost-effectively meet those standards. While making these significant changes, we remain committed to maintaining or exceeding our past performance on traditional measures of environment, safety, and health outcomes.

Third, we must meet DOE's and the public's expectation for cost-effective operation. We must significantly improve our productivity and costeffectiveness, becoming one of the highest value providers of science and technology in the world. Accordingly, we are committed to becoming the benchmark case for management of national laboratories in terms of value of the work we do; the quality of the working environment; our organizational effectiveness; the stewardship of our human, physical, and intellectual assets; and our cost-effectiveness. In 1995 we initiated a major, multiyear productivity enhancement initiative, Achieving the Competitive Edge (ACE). Under this program we are examining the value of our products, both those we deliver to customers and those we produce in our management of the institution or in response to DOE requirements. Detailed and comprehensive information on the processes and resources used to deliver has been collected. We are determining the costs associated with these products and the benefit they provide and identifying opportunities to enhance the performance of our research, administrative, and management processes. We are achieving a significant increase in the fraction of total staff time devoted to research and development, accelerating the delivery of new technical products, decreasing the cycle time associated with administrative and management functions, and ensuring effective stewardship of the Laboratory's people, facilities, and programs. By the beginning of FY 1996 we anticipate substantial tangible progress.

Because of the fierce cost pressures to which we are all responding, we must place particular emphasis on the stewardship aspect of this objective. Apparent increases in productivity obtained by reducing cost through failure to reinvest or failure to develop new capabilities are counterproductive. Efficiency improvements in needed activities and elimination of unnecessary or low-value activities will provide the needed productivity increase. However, in making these improvements we must take great care not to compromise our ability to respond to current and future national needs. We are, therefore, committed to increasing our investment in Laboratory Directed Research and Development.

Finally, we must respond to what is a key theme in the Department's Strategic Plan. We recognize that our highlevel objectives require a foundation of effective communication and trust, both within our institution and between us and our customers, partners, and the general public. Continuous, rapid change in the world to which we respond and in our own business and management practices is inevitable. If our staff are to successfully respond to these changes, they must have confidence that the Laboratory is home to open, honest communication. If our customers and partners are to continue to entrust us with significant resources and shared responsibility for their future, they, too, must have confidence in both our character and our competence. Each staff member, whether working in research, in administrative functions, or in management, is both accountable for, and entitled to, this foundation.

\section{Fundamental Strategies}

In this section we present a set of strategies-how we will achieve our objectives-that we believe respond to the trends presented above.

It is our responsibility to deliver recognizably high-value science and technology to the nation, at reasonable cost, while maintaining the technical capabilities of the Laboratory against future needs. Thus, we ask ourselves, given our set of planning assumptions, what strategy will ensure that we provide high value to the nation and DOE, in both the short and long term? How, in this planning context, can we meet the twin challenges of relevance and productivity?

We believe that there are five broad, closely linked and enduring strategies critical to our future.

- We will deliver high-value solutions to DOE's most pressing and complex issues and technical challenges. Over the last few years we have attempted to more tightly focus the Laboratory's programs around the environmental mission, and those elements of DOE's other missions that best match our core competencies. In our chosen focus areas we will set and achieve high-level objectives that are of real value in meeting DOE's and the nation's most critical challenges. 
- We will build and maintain an outstanding fundamental science capability, directly and effectively linked to the nation's highest priority environmental needs and policy issues. When we made the transition to being an Energy Research laboratory, we made the commitment to establish a fundamental science capability fully on par with our traditional engineering and applied science strengths. Through Laboratory Directed Research and Development and our programmatic investments, we have developed leading-edge fundamental science programs and capabilities in selected areas of molecular, environmental, and life sciences that we believe are particularly relevant to the Department's environmental mission. We will continue this investment, with heightened emphasis on the direct linkage of our basic science capability to DOE's missions and national needs.

- We will maintain and enhance a focused set of core competencies, relevant to both current and emerging national needs. Over the past few years, we have begun to manage the Laboratory's technical capabilities around a set of three integrating or core competencies: Integrated Environmental Research, Process Science and Engineering, and Energy Systems Development. We continually review both the health of these competencies and their relevance to current and anticipated national needs. Changes to our capabilities portfolio will be made in line with our understanding of those needs.

-We will bring the full technical resource of the nation to critical problems through broad partnership with our sister national laboratories, and with universities and industry. The breadth and complexity of DOE's missions make it unlikely that any single institution will maintain the complete set of core competencies required to respond fully to mission needs. We believe we can best meet the Department's needs for comprehensive solutions to complex problems by building partnerships that draw on the most appropriate resources of the laboratory system, our universities, and U.S. industry. Major programs that we have participated in initiating over the last several years, such as the Atmospheric Radiation Measurement program, The AMTEX Partnership, and several of our major environmental remediation programs reflect this practice. We have also made significant progress in simplifying the mechanisms by which we can create suitable partnerships, particularly with academic researchers. We will continue to make partnerships a way of life.
- We will dramatically enhance our technical productivity while protecting the health and safety of our staff, the public, and the environment. Both DOE requirements and our duty to the public and taxpayers require that we achieve cost-effective excellence in our operations, fully meet the Department's expectations for conduct of operation and deliver the highest possible return on the nation's investment in our programs. We are nearing completion of a thorough, in-depth review of our research, management, and administrative and operation practices, and reorganized to strengthen mission focus and to reduce management costs. In cooperation with DOE, we expect to achieve dramatic operational improvements within the next year.

On the basis of this strategy, we have a bright future. Our science and technology is and will remain highly relevant to important national needs. We are fast becoming one of the most productive and cost-effective laboratories in our country. As a result, we are confident that Pacific Northwest National Laboratory will be making significant science and technology contributions well into the next century and beyond. 


\section{Laboratory Initiatives}

Initiatives are the primary means by which we enhance our scientific and technical capabilities to support the U.S. Department of Energy. We are proposing seven multidisciplinary science and technology initiatives for DOE's consideration. ${ }^{(a)}$ Investment in these initiatives will build new capabilities and strengthen existing core competencies. These initiatives, built on past accomplishment and our accumulated skill, show our focus for the future.

The proposed initiatives described in this section directly respond to the missions of DOE in energy, science and technology, and the environment, and linkages between these missions. Consistent with the Pacific Northwest National Laboratory's mission, most of the proposed initiatives are directed toward the interface between the science and technology and environmental quality missions. As shown in the figure, the seven Laboratory initiatives directly address three of DOE's mission areas. The initiatives are

- Molecular Sciences Research (MSR). This initiative is advancing the state of the art in molecular sciences, and applying the knowledge gained from these advancements to DOE missions, particularly the environmental quality mission. It is the focal point of our efforts to enhance the fundamental science base that supports our core competency in integrated environmental research. The principal research thrust areas are chemical structure and dynamics, theory modeling and simulation, materials and interfaces, macromolecular structure and dynamics, computing

(a) Initiatives are provided for consideration by the Department of Energy. Inclusion in the plan does not imply Departmental funding, or intent to implement any initiative. and information sciences, and environmental dynamics and simulation.

- Microbial Biotechnology (MB). The Microbial Biotechnology initiative is developing an integrated scientific and engineering capability in microbial systems and applications. This capability is enhancing our core competency in integrated environmental research and is establishing the foundation for a broader role for the Laboratory in biotechnology. Specific research areas incorporated in the initiative include microbial diversity; microbial genetics; enzyme structure/function enzyme redesign; informatics; bioprocess engineering; and ethical, legal, and social implications research.

- Global Environmental Change (GEC). Global Environmental Change is directed toward developing an integrated understanding of the global impacts of energy production and resource utilization and related complex environmental and policy issues. The initiative is enhancing interdisciplinary capabilities for understanding and modeling the coupled socioeconomic, physical, and biological systems that make up our environment. Specific research areas include regional climate modeling, modeling the effects of climate change on water resources and unmanaged ecosystems, heterogeneous atmospheric chemistry, modeling of emissions levels for greenhouse gases, and analysis of the economic and other social impacts of global environmental change.

- Complex Modeling of Physical Systems (CMPS). This initiative is directed toward providing a greatly enhanced capability for computational modeling of complex physical systems through the use of massively

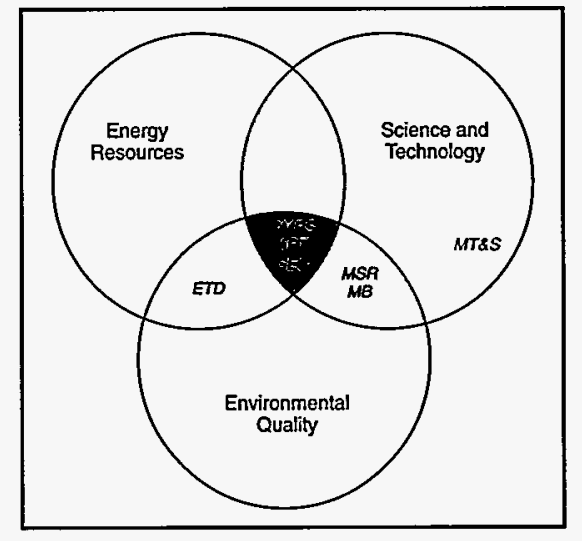

Our initiatives support DOE's environment, science and technology, and energy missions.

parallel computing architectures and advanced numerical methods. The capability will be represented by efficient, state-of-the-art computational models that utilize parallel systems. Applications include global atmospheric chemistry modeling, groundwater transport modeling, computational fluid dynamics modeling, modeling of turbulent flow, and marine transport.

- Advanced Processing Technology (APT). The Advanced Processing Technology initiative is focused on enhancing our core competency in process science and engineering. Primary thrust areas include 1) advanced processes, including chemical conversion and separations; and 2) advanced materials, which include advanced coatings and materials synthesis and processing. We are also using this initiative to develop the infrastructure and culture necessary to more rapidly advance processing technologies through the development cycle. Technical thrusts have been selected based on two principal application targets: tank 
waste processing and environmentally conscious processing.

- Energy Technology Development (ETD). The Energy Technology Development initiative is designed to enhance the Laboratory's core competency in energy systems development. Current research activities are focused in four interrelated technology areas: microtechnology, transmission and distribution systems, advanced operations and maintenance technology, and transportation materials.

- Medical Technologies and Systems (MT\&S). The Medical Technologies and Systems initiative is focused on applying our existing multidisciplinary capabilities to the development of innovative new technologies in health care. The initiative is supporting the efforts of DOE's Office of Health and Environmental Research to contribute to national needs in this area. Current focus areas include advanced ultrasound, medical sensors, medical informatics and computation, targeted therapeutics, medical materials, and medical process improvements.

\section{Molecular Sciences Research}

The Molecular Sciences Research initiative is a key component in the Pacific Northwest National Laboratory's effort to continuously develop and maintain the fundamental science base that is necessary to support the Laboratory's environmental mission and DOE's strategic objectives. The objective of the initiative is to advance the fundamental knowledge of the molecular sciences and to apply the knowledge gained from these advancements to environmental quality and fundamental science and technology issues of importance to DOE. The Molecular Sciences Research initiative is the basis for development of the research programs that will be conducted in the Environmental Molecular Sciences Laboratory (EMSL), a scientific user facility currently under construction at Pacific Northwest National Laboratory.
Through this initiative, the Laboratory is developing the fundamental understanding of molecular structure, interactions, and dynamics in the complex molecular systems that is important to DOE businesses. A major focus of this research is to help create links between molecular-level understanding of condensed-phase and interfacial chemical interactions and reactions and new solutions to environmental problems, such as contaminated soils and groundwater and the complex waste stored in tanks at Hanford. Major components of the initiative (e.g., Materials and Interfaces, Environmental Dynamics and Simulation, and Advanced Processing) are specifically directed at linking molecular science to field problems. These research activities will provide the fundamental scientific knowledge needed to develop permanent, cost-effective solutions to the nation's environmental problems as well as to develop environmentally acceptable energy options for the future. Research programs are also being developed to address key national initiatives such as HighPerformance Computing, Biotechnology, Materials Synthesis and Processing, and Global Change.

Collaborative research involving scientists from other DOE laboratories, universities, and industry are key ingredients in this initiative. Programmatic support for this initiative is sought through various DOE offices as shown in the initiatives table at the end of this section. Multiyear resource requirements are partially included in the Resource Projections.

Following are descriptions of the major research programs in the Laboratory's Molecular Sciences Research initiative.

\section{Chemical Structure and Dynamics}

Chemical Structure and Dynamics responds to the need for a fundamental, molecular-level understanding of chemistry at a wide variety of environmentally important interfaces. The research is built around the established relationship between structure, thermodynamics, and kinetics, and involves rigorous studies of fundamental molecular processes in model systems (e.g., well-characterized surfaces, solutions, clusters, and biological molecules), and studies of complex systems found in the environment (e.g., multispecies, multiphase solutions; solid/liquid, liquid/liquid, and gas/surface interfaces; colloidal dispersions; ultrafine aerosols; and functioning biological systems).

Experimental studies of molecular and supramolecular structures and thermodynamics are key to understanding the nature of matter, and lead to direct comparison with computational results. Kinetic and mechanistic measurements, combined with real-time dynamics measurements of atomic and molecular motions, provide for a molecular-level description of chemical reactions. We expect that this work will result in several advancements including achievement of a quantitative understanding of chemical processes at complex interfaces, development of new techniques for detection and measurement of species at such interfaces, and interpretation and extrapolation of the observations in terms of models of interfacial chemistry.

Structural and kinetic studies of phenomena at model oxide surfaces will elucidate mechanisms of the complex interfacial chemistry of the subsurface environment. The research is focusing on the development of model systems whose study will enable direct comparison with theory and provide insight into the structures and kinetics of molecules interacting with environmentally important surfaces.

Studies on the bonding and structure of organic ligands at oxide/water interfaces investigate mechanisms of sorption of organic ligands on mineral surfaces in aqueous environments using sensitive spectroscopic methods and ice models of water chemistry. This research will provide a basis for our expanded research on geologically important mineral surfaces and environmentally significant ligand/ complexant interactions. 
Research into the mechanisms of radiolytic decomposition of complex nuclear waste forms examines the complex mechanisms of radiation(gamma-ray, $\mathrm{x}$-ray, and electron) induced degradation of glasses and ceramics that are used in the long-term storage of high-level nuclear wastes. The emphasis of this work is on understanding the underlying physical and chemical mechanisms responsible for the loss of the integrity of the composite matrix materials.

\section{Theory, Modeling, and Simulation}

Theory, Modeling, and Simulation focuses on combining the elements of theoretical and computational chemistry, materials science, and molecular biology with advanced computing technologies to help provide comprehensive solutions for the environmental, energy, and other missions of the DOE. Research programs have been established in the areas of molecular theory and modeling, solid-state theory and modeling, and biomolecular modeling and simulation. The research effort in molecular theory and modeling is well established (see description under Basic Energy Sciences/Chemical Sciences). Investments are currently being made to further develop the other two research areas.

One focus of the research in solid-state theory and modeling is on understanding how the molecular properties of clay minerals directly affect mineralsolution equilibria and the transport of contaminants through the subsurface. Current programmatic research is focused on the physics and chemistry of these materials and on the interaction of these materials with selected chemicals. The research initiative in this area includes a project to understand and predict the solid-solution chemistry of oxides, silicates, and carbonates that are important in the environment using first-principles calculations. This study is focused on calculations of the equations of state of oxides, silicates, and carbonate components.

A second focus of the research is on understanding how molecular properties of zeolites, metal oxides, and pyrophosphates influence catalytic chemistry. Current programmatic research is focused on the catalytic chemistry of zeolites and pyrophosphates. Investments are being made to understand the influence of surface geometry and electronic structure on the reactivity/selectivity of heterogeneous chemical catalysts. This theoretical study is focused on metal oxides that are being studied experimentally.

Research in biomolecular modeling and simulation emphasized the structure and chemical function of complex biomolecules. Investments are being made to extend the research effort in this area to study the dynamics and design of protein-DNA complexes. The primary objective is to understand the molecular damage to DNA resulting from exposure to chemicals and the mechanisms by which enzymes recognize and repair damaged DNA.

To provide the molecular modeling software required to meet the computing demands of the research programs, Theory, Modeling, and Simulation contains a research and development effort in molecular science software. This effort is focused on the development of software systems that take advantage of emerging computer technologies and advanced computer architectures for modeling the complex molecular systems found in the environment. This includes exploring innovative approaches to describing electron correlation in molecular systems, such as the numerical solution of the Schroedinger equation.

\section{Materials and Interfaces}

Chemical and physical processes that take place in the boundary regions between solids and liquids, two immiscible liquids, gases and solids, and gases and liquids play key roles in solving environmental restoration and management problems. They control such phenomena as the rate of transport of chemicals between these phases, the rates of transformation in catalytic conversions, and the dynamics of permeation of species through the environment. A detailed understanding of the interactions that take place between a solid and a gaseous or liquid environment are also

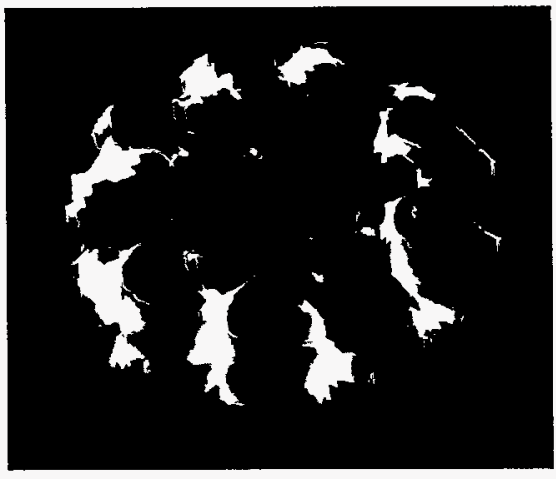

Researchers at Argonne developed a process that uses a crown ether $(K+/ 18$-crown- 6 ether shown) to separate strontium-90 from acidic aqueous solutions. EMSL researchers' modeling efforts are saving enormous amounts of time and improving the performance of the ligand.

essential for rational design of chemical sensors that are needed in any environmentally related activity. Sensor research thus logically falls into the area of physical chemistry of complex systems.

Surface chemical bonding differs significantly from bulk material bonding. A current effort measures surface chemical bonding in environmentally relevant materials to understand how this bonding influences the character and rates of surface reactions that involve water and adsorbates of various types (e.g., organics and metal ion complexes). Research has begun with the simplest, most well-defined, environmentally relevant crystallographic structures (mineral carbonates, metal oxides) where molecular theory and spectroscopy are immediately applicable. Research will then progress to materials with more complex structures, such as iron and titanium oxides with substitutional impurities. The photo-electrochemical properties of titanium dioxide have emerged as a nucleus of much broader interphasial research activity. We recently discovered that catalytic destruction of organic molecules can occur when the catalyst (titanium dioxide) is irradiated with high-energy $(\mathrm{MeV})$ photons. We are collaborating with other researchers in U.S. industry and universities to advance this concept. 
Epitaxial materials synthesis will address problems related to the molecular-level understanding of failure mechanisms in protective ceramic coatings. Model metal/ceramic interfaces will be prepared by molecular beam epitaxy and chemical vapor deposition. These systems will be investigated in great detail using both optical and charged-particle probes to understand the details of interface bonding and adhesion and the role in thermoelastic and environmental stress in initiating failure.

Chemical sensor research focuses on development of advanced sensing layers for the design of higher order chemical array systems. When supplemented with chemometrics, such systems are capable of operating with superior performance in demanding environments. Systematic development of new selective polymer layers is based on the study of fundamental interactions between polymers and organic vapors. Flow injection analysis has proved to be an efficient approach to analysis of real radioactive samples, specifically, strontium-90 in tank waste.

\section{Macromolecular Structure and Dynamics}

Research in macromolecular structure and dynamics focuses on health and environmental issues of interest to $\mathrm{DOE}$ with a strong emphasis on assessing health risks from environmental pollutants and understanding pathways by which human exposure to these pollutants occurs.

One of the grand challenges of molecular biology is to determine the relationship between molecular structure and function (i.e., structural biology). The basic research objective of our structural biology research is to determine the structure and dynamics of native and altered biomolecules that are important for human health. These projects emphasize biological macromolecules, proteins, and nucleic acids involved in regulation and control at the cellular, tissue, or organism level that are not amenable to normal x-ray structure methods or use other spectroscopic techniques which complement those methods. We are concerned with enzymes that produce chemical messengers or that recognize and repair altered molecules. We are interested in how cells recognize and respond to exposure to toxic chemicals and/or radiation. In particular, the interrelationship between the cellular response and the chemical changes introduced in the proteins and nucleic acid will be established. This work relies on stateof-the-art magnetic resonance and mass spectroscopic techniques to study small amounts of natural and modified biomolecules prepared in the laboratory or isolated from biological systems.

Chemistry in the environment is basically chemistry at interfaces. Therefore, an objective of interfacial chemistry research in the macromolecular structure and dynamics program is to determine structural details (selected bond distances, angles, and spatial distributions), and dynamics (rotational and inferences concerning translational diffusion) of small molecules adsorbed to oxide surfaces, metals on supported surfaces, and clays.

Instrument development is an essential component of the Macromolecular Structure and Dynamics program. Several developmental projects are under way, including the construction of an ultrahigh-field nuclear magnetic resonance spectrometer $(900-$ $1000 \mathrm{MHz}$ ) and selection of pulsed and continuous wave electron paramagnetic resonance spectrometers ranging in frequency from 2 to 220 gigahertz. The need to study environmentally relevant samples and materials drives the development of electron paramagnetic resonance instrumentation that will be housed in the EMSL. The operating fields and frequencies are chosen to suit the sample rather than choosing the sample to suit the spectrometer. Consequently, we are developing pulsed and continuous wave electron paramagnetic resonance spectrometers at different frequencies to study different problems.

Nuclear magnetic resonance methods can play an important role in waste characterization and risk assessment associated with the environmental cleanup efforts at Hanford and other
DOE sites. We are exploring the application of nuclear magnetic resonance techniques to characterize the waste in tanks. Techniques include 1) ex situ tank waste characterization, analyzing samples from core samples (i.e., the amounts of free moisture, bound moisture, soluble and insoluble organics, metals, nitrites, and nitrates will be determined); 2) in situ, and potentially continuous, tank waste characterization, using special equipment that will be lowered into a hole in the waste to monitor the free moisture concentration, not unlike magnetic resonance well-logging technology used extensively in the petroleum industry; and 3) process control, where nuclear magnetic resonance methods will be used to monitor, regulate, and optimize the processing of the tank waste during cleanup operations. Characterizing the materials in the waste tanks is the first step in any risk assessment program associated with cleanup.

Development of the 23.5 tesla magnet for the ultrahigh-field nuclear magnetic resonance spectrometer presents some significant opportunities for advancing the state of the art in experimental nuclear magnetic resonance spectroscopy. However, to make efficient use of the sensitivity and resolution that such a spectrometer will afford, novel probes (both for liquids and solids) will have to be designed and fabricated. Modern biochemical and solid-state nuclear magnetic resonance probes require the use of double and triply tuned probes, all with variable temperature capabilities. Additional development work is planned around the need for in situ analysis of solid catalysts. This kind of in situ analysis is made possible by special solid-state cross polarization/ magic-angle spinning (CP/MAS) nuclear magnetic resonance probes.

EMSL staff and other Pacific Northwest National Laboratory scientists are collaborating in efforts to advance the state of the art of mass spectroscopy technology. These advances, which involve improving the sensitivity and resolution of mass spectrometers, are essential in order to address many 
significant environmental problems. Each order of magnitude improvement in the resolution and sensitivity brings about a quantum advance in the problems that can be investigated using mass spectrometry. Our goal is to address important problems that cannot be addressed by today's analytical methods. Therefore, instrumentation and techniques are being developed for the ultrasensitive characterization of extremely small sample sizes with an emphasis on large molecules (e.g., biopolymers). The development of these vastly improved analytical methods is dependent upon other research activities that are focused on gaining a detailed understanding of ion motion and interactions in ion traps and on the dependence upon the details of the trap design. Another area of study involves the chemistry from which detailed structural information for large molecules can be derived. New methods based upon ion-molecule reactions, laser photo-dissociation, and dissociation processes induced by collisions will be developed for structural characterization of large molecules.

\section{Computing and Information Sciences}

Computing and Information Sciences is focused on developing pilot and prototype implementations of the next generation of scientific computing tools and infrastructure, research information products, and instrument designs and data systems for the EMSL. Efforts are aimed at demonstrating the feasibility of new approaches, technologies, and concepts.

Collaborations are critical to advancement in all scientific fields. A cornerstone of the computing and information sciences research is to take advantage of emerging networking and computer technologies to enhance collaborations. Initial efforts are focused on 1) development of tools and expertise needed to enable and implement computer-facilitated remote collaborations; 2) identification of social, systemic, and technological issues that must be addressed to support the dynamics of scientific collaborations; and 3 ) implementation and evaluation of prototype collaborative environments for nuclear magnetic resonance and chemical physics.

New programs are also being developed in several advanced scientific software areas. Research in rapid response sensing applications employs neural network technology to recognize patterns among complex sets of data inputs. Another effort is extending the capabilities of molecular visualization via graphics tools for high-performance parallel computers. Computer modeling of instrument performance also provides significant insights in the optimization of instrument designs. Modeling of next generation Fourier transform ion cyclotron resonance mass spectrometry (FT-ICRMS) instruments is contributing to designs capable of making measurements an order of magnitude more precisely than previous instruments.

\section{Environmental Dynamics and Simulation}

Environmental Dynamics and Simulation emphasizes developing molecularscale information on the structure and reactivity of aqueous and gaseous complexes on solid surfaces and in complex fluids, and linking knowledge of molecular mechanisms to chemical transport phenomena occurring on the pore-scale and microscopic-scale in aqueous, gaseous, and porous media. The effort relates molecular interactions in homogeneous and heterogeneous phases and at interfaces to their phenomenological expression in the environment and brings to bear the capabilities of environmental spectroscopy and advanced computational modeling on the chemical phenomena that affect contaminant fate and transport in various media. The knowledge of the nature of molecular mechanisms resulting from this approach provides information to adjust and expand existing approaches to modeling electrostatic interactions at aqueousmineral interfaces and to improve chemical modeling associated with atmospheric dynamics.

Research in Environmental Dynamics and Simulation contributes to DOE's environmental mission by elucidating the individual contributions of molecular species of varying structure and energetics to volume-averaged chemical behavior observed in bench-scale experiments of chemical fate and transport in natural systems. Improved models of microscopicscale processes based on the molecular perspective are relevant to a number of research and development programs that address scaling from microscopic to macroscopic dimensions. The majority of the research activities are focused on reactivity of the aqueous-mineral interface, with smaller efforts in complex fluids and solutions, bioremediation and enzymatic reactions, advanced characterization, and atmospheric chemistry.

Research is under way to study the interactions between organic ligands and metals. Naturally occurring organic ligands play an important role in soil-pore water equilibria. These ligands influence the weathering of soils and the mobility of metals. The stability of a limited number of metal chelate complexes has been determined experimentally, and semiempirical schemes have been developed to predict the stabilities of certain classes of complexes. These studies have suggested that the steric strain accompanying complexation is an important factor in determining complex stability. The work is aimed at developing a quantitative understanding of the relationship between structure and reactivity of organic ligand/metal complexes. This work is currently being extended to include organic ligands that have potential to selectively extract radionuclides from waste streams.

Another important aspect of environmental dynamics and simulation is the development of expertise in the application of synchrotron radiation to geochemical problems. Synchrotron sources offer very intense, highly collimated, tunable, and essentially monochromatic radiation in the $\mathrm{x}$-ray, ultraviolet, and infrared spectral regions. Our researchers have performed preliminary $\mathrm{x}$-ray absorption spectroscopy (XAS) studies of lead and nickel compounds at the National Synchrotron Light Source at 
Brookhaven National Laboratory. This research attempts to distinguish among the oxide, hydroxide, carbonate, and hydroxy-carbonate forms of these elements to help establish which of the forms were responsible for controlling aqueous concentrations in groundwater. Other $\mathrm{x}$-ray absorption spectroscopy research involves measurements of the structural environments of neodymium (an analog of americium) in carbonates, chromium in contaminated soils, and iron at different redox states in clay minerals. Laboratory staff are also continuing their participation in the PacificNorthwest Consortium beam-line proposal for the advanced Photon Source at Argonne National Laboratory.

\section{Advanced Processing}

The EMSL Advanced Processing program is designed to link Pacific Northwest National Laboratory's molecular science research to the real problems of waste cleanup by providing a pathway for developing scientific ideas and concepts into processes that address DOE's needs, particularly at Hanford. This program is linked to the Laboratory's broader Advanced Processing Technology initiative.

The nation is faced with the immense task of treating and managing highly toxic byproducts and wastes that include complex mixtures of organic and inorganic chemicals and, in some cases, radioactive materials. Carcinogenic chlorinated hydrocarbons, often mixed with various radionuclides, are one of the most significant contaminants in soils and groundwater at DOE and U.S. Department of Defense (DOD) facilities. The current lack of established de minimus standards for such mixed wastes requires that these contaminants be completely isolated and/or destroyed. Incineration, one of the most commonly proposed methods of handling hazardous waste, can create significant secondary environmental problems. Properly designed, heterogeneous catalytic oxidation or incineration processes combined with catalysis can eliminate such problems. The development of these new technologies, in turn, requires a sophisticated process science program with the ability to design and test new catalytic materials and facilitate their full implementation into waste processing systems. As the wastes to be dealt with are identified and characterized, catalysts that are compatible with both the waste and the chosen treatment process stream must be developed and tested.

To accomplish this level of compatibility, new catalysts must be created by rational design rather than the currently used trial-and-error process, which is very time intensive. Rational design of an effective catalyst requires research that leads to an understanding of the catalytic reaction mechanism(s) and the design and synthesis of complex, composite materials. This research must include the disciplines of surface and materials chemistry and molecular and materials modeling.

Another area of major concern is the technical uncertainties associated with the current baseline for remediation of the waste stored in 177 underground tanks at Hanford. The source of these uncertainties is the complete heterogeneity of the waste, both within a single tank and among different tanks. The waste exists in a highly alkaline $(\mathrm{pH}>12)$ environment as liquid supernate, slurry, solid salt cake, and sludge, and little is known about the chemical speciation and phases present in the tanks. It is one of the most challenging problems in chemical processing that has ever existed, and it demands the development of advanced processes for 1) separations to remove the relatively small amounts of radionuclides from the bulk of the chemical waste, 2) conversions to remove those constituents that interfere with the vitrification of the low-level and high-level waste, and removal of hazardous process off-gases from the environment, 3) retrieval of the waste from storage tanks and transport to the processing plants, and 4) design of chemical and physical sensors to provide in situ, real-time information on the waste stream for process control.

It is also necessary to design materials that can operate in several chemical and radiolytic environments. This must be done in a rational way, based on a solid, scientific understanding of the fundamental chemistry and physics that underlie these processes.

\section{Microbial Biotechnology}

The overall goal of the Microbial Biotechnology initiative is to build a comprehensive biotechnology capability that significantly contributes to DOE's core businesses in energy, national security, health, and the environment. This initiative is initially focused on fundamental investigation of microbial systems through directed research leading to microbial applications. Strategic investments in new staff and integrated scientific and engineering programs are creating new state-of-the-art capabilities in basic research to understand microbiological phenomena and to develop and test novel concepts for advanced bioprocesses based on fundamental knowledge.

In the next century, biotechnology will dramatically impact all facets of society and the economy as a consequence of the rapid accumulation of fundamental knowledge of biological systems resulting from federal and privatesector investments in molecular and cellular biology, human and microbial genome sciences, computational and structural biology, and advanced engineering processes. Diagnosis and treatment of disease, environmental stewardship, materials, industrial processes, and ultimately, cultural and ethical values will all undergo major transformations. To address these trends, the Laboratory strategy in biotechnology is to enhance its research programs in life, environmental, materials, chemical, and computational sciences through selective investments to build and integrate capabilities in structural biology, microbiology, enzymology, genome sciences, biochemical and bioprocess engineering, and ethical, legal, and social issues. The resulting theoretical and experimental base, coupled with new capabilities in the EMSL, will result in the successful development of new interdisciplinary programs in fundamental and applied microbiology that are directly linked with DOE missions and the growth and development of the biotechnology industry. 
Pacific Northwest National Laboratory's Microbial Biotechnology initiative is focusing initially on fundamental microbiology (molecular biology, microbial genome mapping and sequencing, bacterial enzymology, physiology and metabolism), microbial and molecular applications in key areas (bioremediation, biotreatment of industrial waste streams), and technology planning and analysis (risk management, economic and social issues).

Fundamental research addresses the following issues:

- Isolating and characterizing microorganisms from unexplored and extreme environments to determine their roles in natural biogeochemical processes, to understand their origins, and to examine their potential for new industrial processes. Target microorganisms include deep subsurface bacteria that may have survived for long periods in ancient depositional environments under oligotrophic conditions, as well as thermophiles, halophiles, barophiles, acidophiles, and anaerobes.

- Mapping and sequencing the genomes of industrially and environmentally important bacteria to provide the basis for understanding and enhancing important and novel functions. Laboratory investments have recently resulted in derivation of a physical map for a 180-kilobase megaplasmid in Sphingomonas, a bacterium from the deep subsurface. The map was used to locate genes encoding the metabolic capability to degrade a unique range of toxic aromatic compounds. The entire plasmid is currently being sequenced.

- Elucidating the structure and function of important enzymes that act as novel biological catalysts and of biomolecules that control critical biogeochemical processes in the environment through integrated research in computational and structural biology. Nuclear magnetic resonance spectroscopy is being used to determine the structure of tetrachloro p-hydro quinone reductive dehalogenase

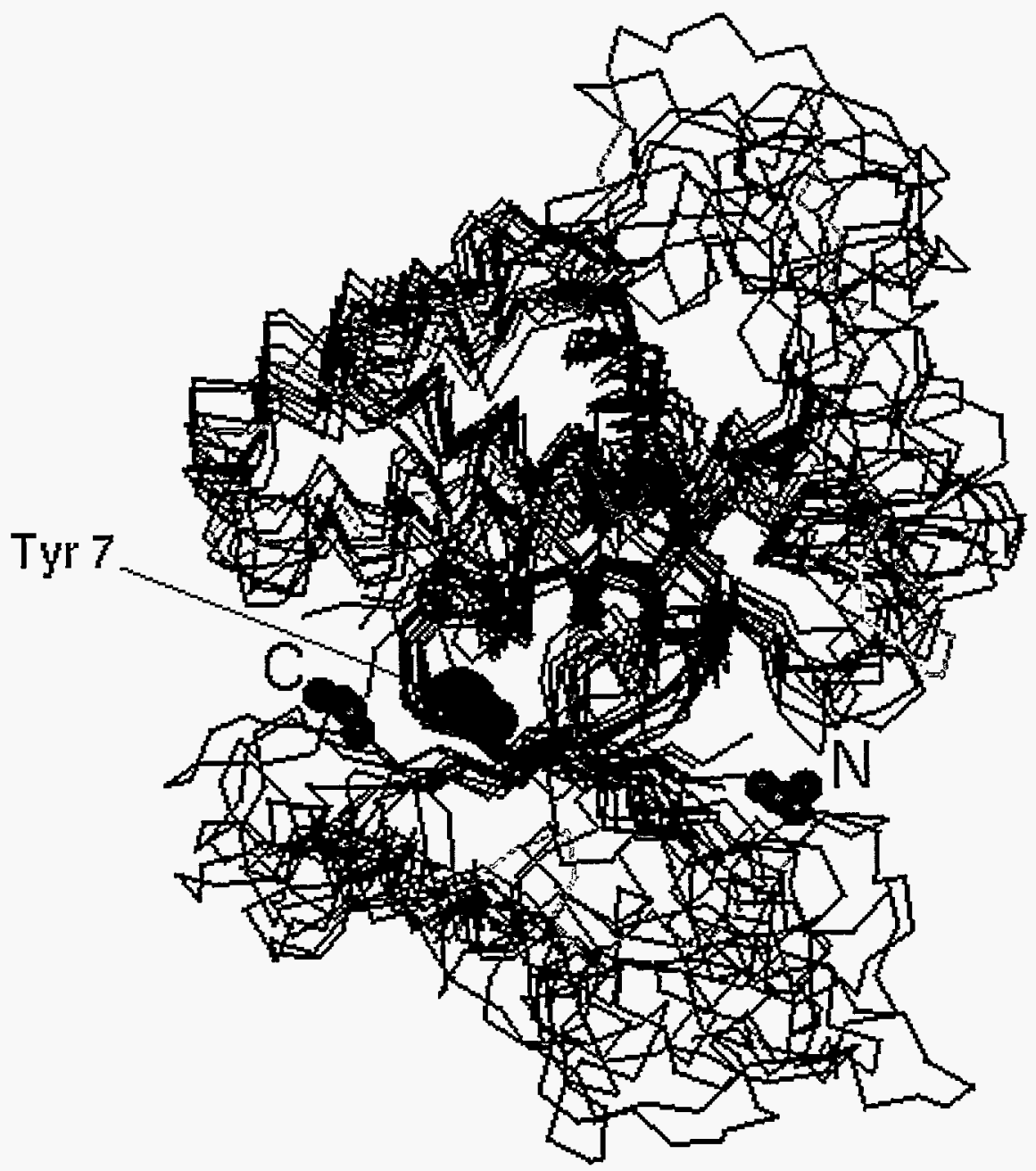

The three-dimensional structure of bacterial tetrahydroquinone reductive dehalogenase (TeCH-RD) has been predicted by distance geometry and homology modeling. The calculations show that the TeCH-RD structure contains both highly conversed and highly divergent structural regions compared to known mammalian glutathione S- transferase proteins.

purified using an $E$. Coligene expression system from a bacterium capable of degrading chlorinated compounds. Molecular modeling tools are being employed to better understand relationships between the structure of thermophilic enzymes (e.g., bacterial subtilisins) and their catalytic activity at high temperature and in nonaqueous solvents.

- Developing interactive links with microbial biochemistry, genetic, and genomic databases on the Internet to provide and access integrated information on fundamental microbial processes and their applications.
- Identifying phylogenetic and functional molecular probes in bacterial dehalogenase and metal reductase systems. This will contribute to a more accurate understanding of biological and ecosystem processes and their response to manipulation of in situ geochemical and hydrological processes for bioremediation.

- Identifying and assessing key social and economic issues, including comparative impacts of competing technologies, environmental stewardship, and ethical and risk perception issues that will accompany the commercialization of new microbial biotechnologies. 
Fundamental research is serving as a basis for new technologies which are examined through joint scientific and engineering research. Opportunities under investigation include the following:

- Novel biological methods for improving the range and efficiency of energy production and chemical processing (e.g., high-temperature biocatalysis), for selective concentration and conversion of wastes and environmental contaminants, and for supporting an improved understanding of global climate change (e.g., biogeochemical cycles).

- Microbial systems for cost-effective treatment of metals and radionuclides and chlorinated hydrocarbons in contaminated aquifers and industrial waste streams through advanced bioprocesses based on enzymatic alteration of valence states and reductive dehalogenation. Initial focus is on iron-reducing bacteria for uranium bioprecipitation and chlorinated hydrocarbon degradation (i.e., mechanisms, kinetics, engineering scale-up).

- New concepts for the use of natural processes in environmental restoration (e.g., bioremediation) resulting from the identification of key physiological, enzymatic, and metabolic properties of microorganisms. Laboratory investments are focused on understanding the mechanisms and genetic control of metal reduction, dechlorination, and degradation of aromatic compounds. The Laboratory has recently isolated the first dehalogenase from a bacterium capable of using dehalogenation to derive energy for growth.

- Rational protein engineering to derive novel concepts for genetic engineering to improve industrial enzyme technology, and for the identification and selection of new biocatalysts from nature to enhance efficiency of chemical manufacturing and waste management processes, such as broadened specificity of bacterial cytochrome $\mathrm{P} 450_{\mathrm{cam}}$ for organic pollutants.
- Basic research in molecular ecology is supporting the development of methods for in situ detection of metabolic activity and the construction of gene probes for specific organisms and activities to improve the performance of field-scale bioremediation research, development, and deployment.

Transferring knowledge and technology to users in the public and private sectors is integral to all aspects of the research. Current efforts encompass transfer to DOE sites, DOE program offices, other federal agencies, universities, and industry. Investments are aimed at 1) development of fundamental capabilities with generic or crossdisciplinary applications (e.g., advances in enzymology with applications in materials, bioprocessing, and bioremediation), 2) integrated research leading to intellectual property and near-term improvements in biologically based tools and processes, and 3) engaging the user community in workshops and colloquia focused on transferring new knowledge and tools and on technological opportunities offered by DOE user facilities, including the EMSL. The growing success of the Laboratory's interdisciplinary approach to technology development and transfer is underscored by a number of research awards and important intellectual property in biotechnology, including four Federal Laboratory Consortium and R\&D 100 awards and over 40 patents awarded and pending.

Funding requirements for this initiative are given in the initiatives table at the end of this section and are partially included in the Resource Projections section. Programmatic support for this initiative is sought through DOE offices with basic and applied research businesses, including microbial origins, microbial genome, and human genome programs in Office of Health and Environmental Research (OHER); the DOE-Environmental Management focus areas, including contaminant plume containment and remediation and mixed waste characterization, treatment, and disposal; DOEINTEL; and the Advanced Industrial Concepts Program in the DOE Office of Industrial Technology. New interfaces with other federal agencies and industry are also rapidly developing.

\section{Global Environmental Change}

The goal of Pacific Northwest National Laboratory's Global Environmental Change initiative has been to develop the understanding necessary to support critical national decisions within the context of global change. The goal is to improve the scientific understanding of the processes and forces shaping the environment and the integration of that knowledge on appropriate spatial and temporal scales to inform government policy and to guide development of technology.

Global change issues develop through interactions between the extremely complex natural biogeophysical system and the rapidly expanding activities of human civilization. In the 20th century, the growth of civilization has progressed to the point that human systems have become major geophysical forces. It has become clear that the production and use of energy represents one of the strongest interactions between civilization and the natural world. The increasing extent to which energy production is being linked to global-scale effects has made global change a central issue in energy policy.

The Laboratory's Global Environmental Change initiative features four elements: 1) contribute to the base of scientific knowledge on environmental change processes; 2 ) provide a framework for evaluating technological responses to global change; 3 ) inform and support the development of policies related to global change; and 4) promote analyses which ensure that the understanding of science, technology, and policy are appropriately integrated with each other. As the initiative nears completion during FY 1996, research is being conducted to support the first two elements. The initiative is based on the premise that a multidisciplinary laboratory is well positioned to meet these needs and uniquely positioned to effectively perform the vital integration function. 


\section{Building the Scientific Base}

The Pacific Northwest National Laboratory scientific research program is well integrated with the entire U.S. Global Change Research Program. This program is developed and coordinated by the Global Change Research Subcommittee of the Committee on Environment and Natural Resources which is an element of the National Science and Technology Council. The program is a U.S. contribution to the International Geosphere Biosphere Program (IGBP). It is described in the report entitled Our Changing Planet: A U.S. Strategy for Global Change Research. The report describes the role the federal government will play in supporting IGBP research to understand both the continuing change in natural biochemical cycles and the disturbances in those cycles that may be caused by human activities.

As a major player in the national Global Change Research Program, DOE has developed programs that are an important part of this major international cooperative effort. Through its support for the Environmental Sciences Division of OHER, the Laboratory has assisted DOE over the past year by active participation in leadership and research in the Atmospheric Radiation Measurement (ARM) and Computer Hardware, Advanced Mathematics and Model Physics (CHAMMP) programs. In addition, the Laboratory contributed significantly to the ocean research element of the Core Carbon Dioxide Research Program. There remains a focus on understanding the formation, removal, and climatic consequences of anthropogenic aerosols and on the development of approaches to detecting impending climate change.

In support of the broad federal objective, as well as the specific DOE business area, the Laboratory has focused its internal investments on the following key areas: regional climate prediction, climate change and its effects on water resources, the effects of climate change on unmanaged ecosystems, and the effect of heterogeneous chemistry on the fate of energy-related pollutants in the atmosphere. The current emphasis is on the linkage of these processes with ecological impacts. Each of these efforts is highly integrated. The goal is to produce a set of linked models that provides a consistent intellectual framework for examining the coupled effects of regional climate change on water resources, unmanaged ecosystems, forests, and agriculture.

\section{Understanding Technology and Global Change}

Technology is an important component of the complex considerations involved in understanding global change. The Laboratory has a strong program that provides basic models of the relationship between energy generation technologies, economic growth, and the emission of greenhouse gases. This effort has been intensified with the ongoing development of a secondgeneration energy/economic model and the development of the Global Change Assessment Model (GCAM).

Global environmental change research integrates the understanding gained from science, technology, and human interactions research, which together define both the global environmental problems and reasonable responses. All of these elements produce understanding that is essential for analyzing the problem and defining effective responses. This understanding is integrated in the form of models that can represent the essential interactions within, and between, the various components. Through the use of these models, areas for potential collaborations with other scientists and institutions are identified.

In addition to developing the secondgeneration emissions model, the Laboratory is attempting to increase the level of understanding about the role of technology in both cause and mitigation of global change. New approaches are required to evaluate technologies based on environmental factors to prevent global change from becoming the basis for unfounded technological advocacy. Our research is aimed at producing a methodology that encompasses all of the direct environmental benefits and consequences of a particular technology, as well as effects that flow from the interaction of different technologies in the marketplace.

\section{Analyzing Policy Options}

The ultimate customer for understanding the global system and civilization's role in it is the policymaker. It is essential that those who consider policy options understand the context in which their decisions are being made. This requires reliable and useful analysis tools to support that decisionmaking process. The Laboratory has created and acquired a set of state-ofthe-art analysis tools related to global environmental issues. The Global Studies Program has used these tools to provide integrated policy support to the DOE Environmental Sciences Division; the Office of Policy; DOEEE; and the National Energy Strategy development. These support activities include

- estimating U.S. emissions and modeling global emissions of carbon dioxide as well as other radiatively important gases

- integrating the multiprogram laboratories' participation in several global policy studies mandated by Congress (such as a report to Congress on options for mitigating carbon dioxide emissions) and supporting the review of such documents

- supporting the incorporation of global change issues in the National Energy Strategy

- developing methodologies such as GCAM to model the impact of regional climate change on natural and unmanaged ecosystems, the economy, and society

- evaluating strategies for carbon dioxide emission mitigation

- evaluation of policy options in support of negotiations under the Berlin Mandate for the Framework Convention on Climate Change.

In anticipation of supporting analysis of future policy concerns, the Laboratory is developing improved economic evaluation tools and methods for evaluating the effect of climate change on human welfare. In particular, we are making a significant investment in the 
development of a model to enable assessments of the effects of energy policy decisions and technology developments on the environment and economies of North America.

\section{Promoting Integrated Understanding of Global Change}

The final element of the Laboratory's Global Environmental Change initiative is driven by the need for integrated rather than piecemeal understanding of the global change issue. A particular emphasis in this area has been on promoting understanding on the international level of the need for national responses to global change concerns. Individual nations both create and respond to global change issues in ways that are coupled to their state of economic development and their resource mix. In general, we are focusing on national responses in the international context as the most appropriate level of integrating responses to global change. The Laboratory's activities have led to

- national studies of carbon dioxide control strategies in support of the Intergovernmental Panel on Climate Change for such countries as Poland, France, the United States, and the states of the Former Soviet Union

- invitations for several scientists from the Laboratory to participate in the 1995 assessment activities of the Intergovernmental Panel on Climate Change

- establishment of an Advanced International Studies Unit in Washington, D.C., to provide a focus for international participation in policy-related programs

- support of the development of the position statement by the U.S. Energy Association related to the study of climate change

- development of a workshop with private industry on living with uncertainties associated with climate change predictions

- establishment of energy-efficiency centers in Russia, Poland, the Czech Republic, Bulgaria, and China to transfer U.S. technology and demonstrate options for reducing emissions of greenhouse gases

- initiation and leadership of an international assessment of the state of the art in the social sciences in terms of the knowledge needed to deal with the social, political, and economic issues of global environmental change.

Funding for the Laboratory's involvement in the Global Environmental Change initiative is sought from various DOE offices and other organizations as shown in the initiatives table at the end of this section. Most of the multiyear resource requirements are included in the Resource Projections.

\section{Complex Modeling of Physical Systems}

Computational modeling and simulation is an essential component of all three of Pacific Northwest National Laboratory's core competencies. It is also critical to the success in many facets of the Laboratory's environmental mission. By enabling researchers to study phenomena that are inaccessible to direct measurement, too costly for experiment, or too complex for analytic theory, computational modeling provides insights into complex environmental phenomena heretofore unavailable. High-speed, largescale computation is already a primary enabling technology in many environmentally related areas, including such diverse fields as

- global climate change

- transport and physical chemistry in the environment, including atmospheric, oceanic, surface and subsurface hydrology, and soil/groundwater interactions

- engineering fluid dynamics

- combustion processes

- materials science

- bioremediation.

In many cases, however, conventional single-processor computers are not large enough or fast enough to carry out studies with sufficiently high resolution and accuracy to achieve the full benefits of computational modeling. The use of computational models will certainly expand as computational power continues to increase at its historical rate of 10 times faster and cheaper every 5 years. For the largest and most demanding problems, however, such increases will be achievable only through the use of parallel computing.

Parallel computing provides the only plausible method for running very large and time-consuming simulations; those requiring 10 to 100 times the performance and memory capacity of a high-end workstation computer. The Laboratory's initiative on Complex Modeling of Physical Systems is a forward-looking effort designed to enable leading edge models to be executed on the full range of computers accessible to modelers, from high-speed desktop workstations to massively parallel computers. This initiative enables continued improvements in modeling capabilities that are important to the Laboratory's environmental mission.

The initiative addresses a wide range of scientific disciplines, including computational chemistry and materials science, environmental chemistry and transport, and engineering fluid mechanics. Extensions to dynamic modeling of the electric power system and to climate research are planned. In each of these technical areas we are following a strategy that includes

- implementation of codes on advanced architecture machines as they become available, both for performance evaluation and for proof-ofprinciple calculations

- performance analysis and modeling of computational algorithms and codes that allow us to both predict performance on potential teraFLOPS machines and to help guide the development of future generation computational hardware and software for scientific and engineering applications

- design of numerical algorithms that capture the full benefits of massive parallelism 
- investigation of selected forefront computational problems that are clearly beyond the capacity of current generation supercomputers.

The centerpiece of this initiative is the development of software technology required for modeling of molecular processes in the environment. Modeling these complex molecular processes requires highly aggressive use of computational technology. Problems that require computational capabilities beyond the current state of the art include

- modeling solvation and reaction in solution to obtain a detailed understanding of the binding of radionuclides to selected ligating agents

- modeling the interactions of ions and molecules with minerals to obtain a better understanding of the binding of contaminants to soil

- modeling the dynamics of biomolecules in solution in order to redesign enzymes to enhance biodegradation of pollutants.

The Molecular Science Computing Facility planned for the EMSL will provide the hardware and initial molecular science software necessary for this work. In 1994, advanced parallel computing and large-scale data management equipment were acquired to support hardware and software development for this facility.

In prior years, this initiative addressed several forefront problems, including

- modeling the effects of spatial variability on subsurface flow in three dimensions (This requires the solution of equations with millions of computational nodes; current generation supercomputers are effectively limited to much smaller problems.)

- modeling the transport and chemical fate of atmospheric contaminants on a global scale

- application of Direct Numerical Simulation techniques to the study of turbulent flow

- full-scale modeling of promising environmental technologies like in situ vitrification and advanced joule-heated furnaces (requiring computing resources far beyond those provided by current generation supercomputers)

- full-scale modeling of waste tank behavior and response to mitigation strategies, such as jet mixing so as to increase the likelihood of success and decrease the chance of catastrophic failures; modeling these time-critical problems requires the use of the most powerful supercomputers available.

Examples of recent significant accomplishments in these projects include

- development of parallel software and completion of a direct numerical simulation study of cell formation and turbulence states in a doublepane window, involving 2.4 million degrees of freedom and 78,000 time steps

- development of a massively parallel version of the Laboratory's Watershed code and use of this code to assess the impact of global warming on the Columbia River watershed

- development of a massively parallel code for modeling subsurface flow and transport in aquifers with multiscale material heterogeneities and use of this code to study permeability scaling behavior in geologically complex groundwater systems

- development of a parallelized version of the Global Chemistry Model (GChM) and use of this code to analyze temporal and spatial distribution of global carbon monoxide compared with space shuttle data

- development of a domaindecomposed version of the TEMPEST program (used for modeling waste tank behavior and other fluid dynamics problems)

- implementation and evaluation of a variety of parallel numerical methods for fluid dynamics.

Pacific Northwest National Laboratory remains an active participant in the Concurrent Supercomputing Consortium. The Consortium, which includes several DOE laboratories, government research agencies, and major research universities, installed the Intel Delta Touchstone supercomputer at California Institute of Technology in May 1991. Significant upgrades in computing capability, including the installation of a next-generation Intel Paragon supercomputer, occurred in 1994. A number of molecular science software packages, including both electronic structure and molecular dynamics programs, as well as coupled atmospheric chemistry and transport and direct numerical simulation turbulence codes have been implemented on the Intel machines. They are now being used to solve problems beyond the practical limit of conventional supercomputers in both molecular science and atmospheric chemistry and transport.

Our collaboration with Intel Supercomputer Division, supported by the Office of Energy Research Laboratory Technology Transfer Program and completed in FY 1995, was highly productive and may serve as a model for future collaborations with other industrial partners. The technology transfer was recognized in FY 1994 with a Federal Laboratory Consortium award for Excellence in Technology Transfer as a result of Intel's commercialization of parallel computing software resulting from the collaboration.

The Complex Modeling of Physical Systems initiative offers opportunities for both undergraduate and graduate students to participate in code development work on advanced architecture computers and incorporates postdoctoral fellows on its technical teams. We are seeking to expand the direct industrial participation in and support for this work to stimulate the application of advanced computer architectures to industrial problems.

In FY 1996 and beyond, we plan to begin and/or continue research in applying parallel computing to several of the scientific domains noted in the introductory paragraph of this section. Final choice of the domains and projects to be funded under the initiative will be determined based on the research objectives of application domain scientists, as they align with developing laboratory missions and strategies. 
Funding for specific elements of this initiative will be requested from DOE program offices directly concerned with the technical problems requiring high-performance computing. Baseline funding for development of highperformance computing techniques is sought from the Office of Scientific Computing. Multiyear resource requirements are given in the table at the end of this section and are not included in the Resource Projections.

\section{Advanced Processing Technology}

Process science and engineering is a core competency that supports the Laboratory's environmental mission. The Advanced Processing Technology initiative is enhancing this core competency by making integrated investments spanning basic research through engineering to rapidly develop and deploy new process technologies for waste treatment and pollution prevention. Technical capabilities in materials and chemical science (colloidal science, solution/solvation chemistry, catalyst development and characterization), molecular science (plasma chemistry, materials characterization), thermal and energy systems (complex fluid behavior), and process technology development (reaction systems for nonequilibrium plasma, electrochemical conversion and separations, supercritical fluid treatment) are being strengthened through this initiative. These investments are ensuring the health and relevance of our process science and engineering core competency.

The Advanced Processing Technology initiative is transforming Pacific Northwest National Laboratory's environmental mission by

\footnotetext{
- Integrating and focusing the Laboratory's process science and engineering investments around two significant national problems: tank waste processing and environmentally conscious processing. Tank waste processing is addressing vulnerabilities associated with radioactive and mixed wastes stored in over 400 waste tanks across the DOE complex. Key technical challenges
}

include the physical/chemical separations of the waste into high- and low-level components and the subsequent immobilization of these waste streams in robust waste forms. The technical vulnerabilities associated with these processes translate into excessive treatment and disposal costs that DOE and the public cannot afford. Investments in the separations and materials synthesis thrust areas are being aligned on this target. Intimate knowledge of tank waste processing needs are obtained through the Laboratory's leadership role in the Tank Focus Area, a national technology development program addressing waste tank remediation issues across the $\mathrm{DOE}$ complex.

Environmentally conscious processing is addressing process substitution, resource recovery, and waste treatment as approaches to reduce environmental and energy impacts for governmental and industrial processes. Our efforts are centered around process substitution where the largest gains in waste reduction and energy efficiency can be obtained. Examples include novel chemical reactors which simultaneously convert and separate products, thereby increasing product yields and reducing downstream separation costs, and plasmaassisted conversion, which uses ions and radicals created from a plasma discharge to promote chemical reactions at lower temperatures, thereby decreasing energy costs. We are also investigating recovery of valuable materials from process streams using a variety of separations techniques and advanced separations materials.

- Implementing and demonstrating methodologies to speed the deployment of process technologies. Major reductions in process development time at the Laboratory are being achieved by a coupled technology development approach-the effective and concurrent integration of basic and applied research, technology development, and engineering activities. As fundamental knowledge of processes grows, the need for mul- tiple scale-up steps diminishes. New processes can then be integrated and demonstrated at much smaller scales, decreasing development time and resource requirements.

Funding requirements for the initiative are given in the table at the end of this section and are partially included in the Resource Projections.

\section{Advanced Processes}

Advanced processes in chemical conversion and separations are required to make major advances in our tank waste and environmentally conscious processing targets. Development of these technologies are supported by advanced materials being developed by this initiative.

Chemical Conversion A key objective of chemical conversion is to use fundamental knowledge of conversion mechanisms to develop novel processes to reduce energy consumption and waste generation, leading to more efficient and competitive chemical manufacturing processes.

Conversion is the process by which chemical reactions alter the molecular structure of raw materials into other molecules of higher value or lower toxicity. In many cases, conversion can only be obtained by using high temperatures or pressures or long reactor residence times. This can consume large amounts of energy and create undesirable byproducts. Our research emphasis is on developing novel techniques which improve conversion processes by reducing the temperature, pressure, and residence time. Our activities are focused in two major areas: 1) nonequilibrium plasma and 2) reaction engineering.

A nonequilibrium plasma occurs when electrons are accelerated in an electric field until they reach sufficient energy to break chemical bonds. This can create reactive species that can be used to destroy toxic compounds or synthesize chemical species at significantly lower temperatures than many conventional conversion processes. This ability may enable the development of very energyefficient and environmentally friendly processes for conversion. Past work 
centered around high-vacuum applications in the coatings area, such as chemical vapor deposition or surface modification. However, application to large-scale conversion processes was limited because high vacuum was required, reducing throughput. Research performed by the Laboratory led to the discovery of a method to create a volumetric plasma at ambient conditions. This enabled the technology to be used for larger-scale processes. Initial work has focused on the destruction of organic contaminants in process off-gas and liquids. Other potential applications include treating automobile exhaust, treating industrial flue gas, and potentially unique conversion processes for industrially significant products such as oxygenated fuels.

The key to successfully applying nonequilibrium plasma is to understand the electric field and the plasma chemistry and to effectively incorporate this understanding into novel reactor designs. To understand the electric field effects, electric field measurements have been taken for various reactor geometries and packings, and a computational model has been developed to assess critical design parameters. To understand the plasma chemistry, a Flowing Afterglow Apparatus has been developed to measure radical and ionic species generated in various plasma configurations. This state-of-the-art diagnostic system can be used to precisely identify chemical species and determine reaction kinetics; a capability which is applicable not only to low-temperature plasma processes but to many other conversion technologies as well. To date, these efforts have led to a fourfold increase in the capacity to destroy organic contaminants in off-gas and unique designs for liquid treatment of organic contaminants.

Reaction engineering is focused on developing innovative reactor designs for conversion. Concurrent development and deployment of novel materials (e.g., catalysts, membranes) are major components of this task and are critical to its success.
Research in this area has centered on inorganic membrane reactors for partial oxidation reactions. Experimental and theoretical investigations show that the membrane reactor outperforms traditional reactor designs by producing both higher product yields and selectivities per pass. For the oxidative dehydrogenation of ethane to ethylene, yields as high as 50 percent per pass are observed in the membrane reactor, where yields less than 10 percent per pass are observed in a traditional plug flow reactor. Selectivity to the desired product, ethylene, is also dramatically higher in the membrane reactor. Higher per-pass yields equate to downstream savings in both energy and raw materials.

New membrane materials with greater capabilities are under investigation. High-temperature permselective facilitated transport membranes are being developed for use in a separativemembrane reactor. Dramatically higher yields per pass will be possible for partial oxidation reactions if coupled with in situ product separation to prevent product overoxidation.

Separations Research efforts in the separations area are focused on processing aqueous waste streams and using supercritical fluids in separation processes. Separation technologies have been explored for the separation of metal ions (toxic and radioactive elements), organic compounds (neutral and charged), and metal complexes (chelated metals and organometallic compounds). The metal ions, cesium and strontium, were targeted for early efforts because of the need to separate these elements when processing tank wastes at Hanford. Efforts have been expanded to other compounds in support of existing and proposed major DOE initiatives such as the American Textile Industry (AMTEX) and Refinery of the Future. The technologies investigated include ion exchange, membrane separations, electroseparations, and supercritical fluid extractions.

Evaluation of the organic ion exchange resins for the separation of cesium from tank waste has centered on elucidation of the primary structure of the resin and examination of synthetic variables affecting the cesium selectivity of the resin. One of the important issues identified during the course of this work that may limit the implementation of these materials is the oxidation of the resin. Current efforts have been focused on preparation and characterization of modified resins that should be more resistant to chemical oxidation. This is being achieved by modifying the backbone structure of the polymer resin. A variety of derivatives are being prepared that may expand the $\mathrm{pH}$ range over which the resin will selectively remove cesium from waste streams.

Electro-separation activities have focused on flexible technologies with applicability to the environmental problems of government facilities and private industry. Electro-separations have the advantage of operating at low temperature, generating minimal secondary waste, and selectively controlling the separation by adjusting the applied potential. The technologies developed include electrodialysis, electroconversion, and electroactive materials. Electrodialysis was used to separate anions such as borate, nitrate, and phosphate. The separation of these ions is important to water-cooled nuclear power plants and spent-acid recovery plants. The application of electroconversion to energetic materials is also being explored. Explosives, such as HMX and RDX, were converted to carbon dioxide, water, or useful byproducts using direct oxidation and reduction. This approach provides an attractive alternative to incineration. Finally, a new capability was developed to prepare, evaluate, and characterize electroactive surfaces. The work focused on preparing thin-film surfaces for selective adsorption and desorption of metal ions by manipulating the electroactive (oxidation/reduction) sites within the film. Films were synthesized on both metallic and conductive-polymer substrates for cesium removal.

Supercritical fluid extraction research has focused on the basic investigation of metal complexation in compressible fluid solvents. Research efforts have 
centered on the development of extraction processes for textile dyes and the removal of metals from aqueous solutions using ligand-impregnated supercritical carbon dioxide. Both of these efforts are predicated on the fundamental understanding of metal complex solubility and chemistry in supercritical fluids. Current investigations have entailed the development of high-pressure nuclear magnetic resonance capabilities to study, at the molecular level, labeled ligands and the metal ion as a function of fluid (solvent) properties. Scale-up of these analytical studies can lead to a closed-loop environmentally benign solvent-extraction process based on supercritical carbon dioxide. This research effort has contributed to the AMTEX initiative in the development of a waterless process for dyeing and sizing fabric based on a "green" solvent-supercritical carbon dioxide. Supercritical fluid extraction technology has many applications in waste water treatment, environmental remediation, mineral recovery, and true "zero-discharge" manufacturing process design. Supporting investigations on the mixing of supercritical fluids essential to the scale-up of any supercritical process are also being conducted.

\section{Advanced Materials}

Advanced materials are critical for breakthrough technologies in the environmental and energy missions. Because of their widespread use, new catalysts and catalyst supports can yield tremendous benefits in terms of environmental impact (increased yields and decreased waste streams) and economic productivity (new products). Advanced coatings offer the opportunity for significant energy impacts via large-scale implementation of light-weight, environmentally hardened materials and new products for energy conservation. New classes of materials, including mesoporous ceramics, organomettalate catalysts, and advanced coatings will be useful for a wide range of DOE applied programs in Energy Efficiency and Fossil Energy, including transportation technologies, industrial technologies, and fuel cell development.
Advanced Coatings The advanced coatings effort is directed toward the development of an integrated capability in thin-film coatings for a variety of applications including energy, environmental restoration, defense, and biomedical. This integration includes state-of-the-art molecular beam epitaxy and chemical vapor deposition capabilities, unique physical vapor deposition, polymer multilayer coatings, and solution-derived, biomimetic thin films. The combination of these coatings technologies provides the Laboratory with a unique range of vapor and solution-based technologies for coating simple and complex geometries-powerful tools to address almost any coating challenge.

The technical and scientific base of the individual coatings components are being strengthened in this initiative. For example, in the biomimetics (solution phase) coatings effort, we are developing a unique scientific understanding of surface-induced thin-film formation. Expertise is being developed in the fundamental understanding of the factors (supersaturation, chelation, solution speciation) controlling film growth in the deposition solutions. Chemical modification schemes that induce biomimetic film growth are being developed for most of the "consumer" plastics (acetal, polycarbonate, nylon, polyethylene, polyvinyl chloride). The ability to deposit ceramic coatings on these plastics has tremendous technical significance.

To strengthen our vapor-phase coatings technology base, we developed a new type of vacuum coating technology (polymer multilayer, physical vapor deposition), while adding more traditional coating processes to our capabilities, including large-scale magnetron and ultrahigh-rate sputtering. An in-line, multilayer, deposition process that lent itself to economic manufacturing and rapid prototyping was developed. Polymer multilayer technology offers a new method for integrating polymer film deposition at prototypic rates and has opened up a whole new area of product applications. A vacuum roll coater, capable of multiple, in-line deposition processes using physical vapor deposition (e-beam and ultraviolet cure), sputtering, e-beam evaporation, thermal evaporation, plasma enhanced chemical vapor deposition, and polymer extrusion and flash evaporation (e-beam and ultraviolet cure) was also developed for rapid prototyping of advanced coatings.

The development of this unique, single-pass system has resulted in a CRADA with DuPont to investigate polymer multilayer/silver films, and both a CRADA and a license agreement with Moltech to develop a lithium-polymer battery. In addition, several other collaborative efforts are under way.

Materials Synthesis and Processing Current efforts center around synthesis and processing of advanced catalytic materials, supports, and devices. Processing of these advanced materials into robust engineered forms is crucial to successful deployment. Projects are also under way on development of new techniques for solid-liquid separations in tank waste processing and in expanding ceramic nanoparticle synthesis to some new composition fields.

In the area of advanced catalytic materials, solid acid catalyst materials are being developed from nanoparticle sulfated zirconia. These materials, having effective $\mathrm{pH}$ as low as -14 (Hammett scale), can be safely held in the hand and will replace large quantities of sulfuric acid that are currently used by the petroleum refineries. Solid acid catalysts will yield significant environmental and safety benefits over the hazardous liquid alternative. A unique Laboratory process for synthesizing sulfated zirconia as a single processing option will facilitate deployment of this new technology.

Organomettalate materials are under investigation for potential replacements to aluminum chloride as catalysts for alkylation and acylation reactions. The aluminum chloride catalysts currently used are "once through" types and create a hazardous waste stream, which has raised strong environmental concerns. The new catalysts that are under development are Lewis acids that can be attached to substrates so they can act as recyclable heterogeneous catalysts. 
Through an investment in mesoporous materials, we continue to explore novel processing routes to synthesize mesoporous materials and nanoscale structures based on self-assembly of ordered structures in surfactant solutions and other organic or biological systems. The self-assembled organic structures function as templates for the nucleation and growth of inorganic porous structures and other ceramic materials in a controlled manner. The inorganic materials produced this way have unique structural order and functional sites which cannot be achieved by traditional processing routes. These materials have specific surface areas in excess of 1000 square meters per gram due to monodisperse porosity. The pore diameters can be controlled in the range of 4 to 20 nanometers by selection of surfactants. The materials developed have direct applications as ion exchangers for environmental cleanup and separation and for catalysis and catalyst supports.

An effort to develop supported metal catalysts is under way for industrially important reactions. This effort builds upon technologies that have been or are under development at the Laboratory (e.g., glycine nitrate combustion synthesis, mesoporous materials) for producing high-surface area inorganic materials. These processes can produce either nanoscale particle size or ultrahigh surface area silica, zirconia, and aluminosilicate materials that offer a unique opportunity for application as supports for metal catalysts. These supports are unique because the high surface area and the large pore volume to surface area ratio inherent in these materials afford the potential for highly dispersed metal deposition.

Methods of processing ultrafine ceramic particles into useful engineered forms are critical capabilities being developed to accelerate the deployment of novel materials into industrial processes. Currently, the project is focused on ultrafine powder consolidation for catalytic applications using both nanoparticle and mesoporous powders. Issues include overcoming problems of consolidating powders that have highly hydrated surfaces, maintaining high surface activity, and fabricating mechanically robust components.

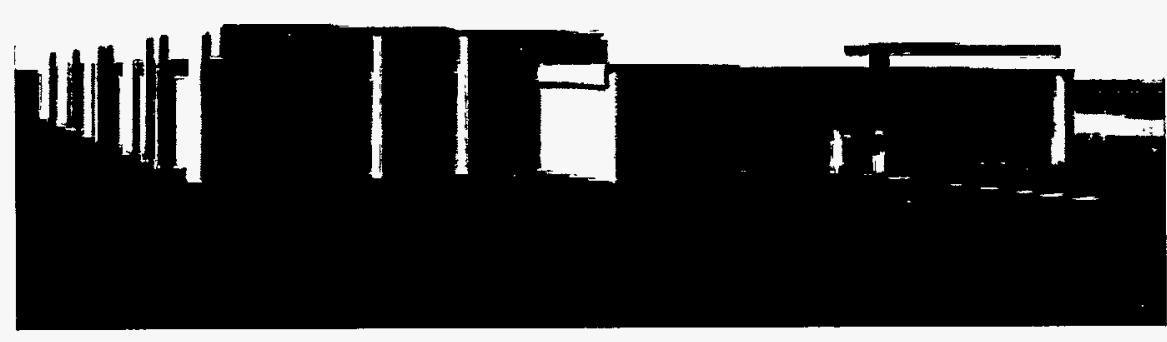

As an engineering-scale user facility, the Advanced Process Engineering Laboratory will be used to develop, test, and deploy new technologies.

These components must retain their high pore volume after processing to facilitate mass transport.

Another effort is focused on developing synthesis of hexaaluminates through cation substitution in the crystal structure and structural modification with a view to designing catalyst materials for high temperature processes such as steam reforming, membrane reactor, and gas turbine catalytic combustion. The objective is to provide an understanding of the relationships between synthesis and catalytic activity, selectivity, and durability.

\section{Advanced Process Engineering Laboratory}

We are supporting, through this initiative, a new effort to build an engineering-scale user facility to rapidly develop, test, and deploy new technologies. Commercial users, other agencies, and Laboratory researchers will use the Advanced Process Engineering Laboratory (APEL) to demonstrate new technologies and to develop the technical and regulatory databases for permitting, licensing, and otherwise establishing the technical, economic, and regulatory basis for applying the technology to real-world environmental and technical problems. The systems and services in APEL include permits; effluent treatment systems; modular configurable support systems; data collection, modeling, and analysis; as well as certified analytical laboratories and a waste treatment/ packaging system.

Funding for this user facility will be obtained through an innovative collaborative funding arrangement with
DOE (Economic Transition Funds), Port of Benton, the City of Richland, and Battelle.

\section{Energy Technology Development}

The Energy Technology Development (ETD) initiative is designed to broaden and deepen Pacific Northwest National Laboratory's fundamental energy systems development core competency. This competency is the primary basis that underlies work for the Office of Energy Efficiency and Renewable Energy, a major contributor to work for the Office of Fossil Energy, and a key element in the Laboratory's collaboration with American industry. In FY 1994, the Energy Programs Directorate led a series of workshops across the Laboratory to refine the focus for this competency. The goal was to establish for the Laboratory, an important niche within the energy technology community; one that can be used to solve important problems, and that readily distinguishes Pacific Northwest National Laboratory from other research and development organizations. A compelling vision emerged from that effort.

The Laboratory will develop and deliver innovative technologies and services that will transform the global energy infrastructure leading to a sustainable energy future. We will focus on improving the use of existing energy assets and developing a new generation of distributed energy systems. 


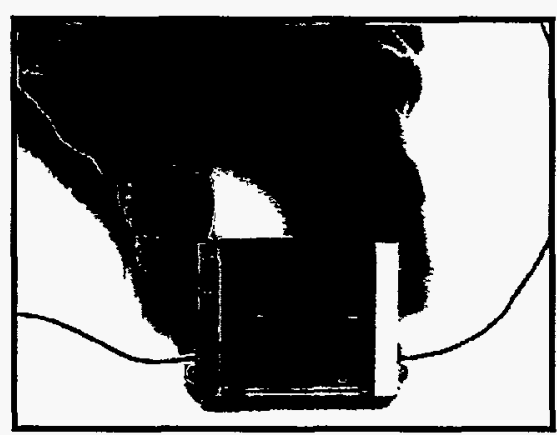

Microscale technology has the potential to reduce production costs.

The key to this vision statement is the dual investment focus on improving the utilization of existing energy assets and developing a new generation of distributed energy systems. These two focuses are highly complementary, and will lead to the development of reliable, cost-effective energy systems that are environmentally friendly and important for maintaining the competitiveness of American industry.

Current research activities are focused in four interrelated technology areas: microtechnology, transmission and distribution systems, advanced operations and maintenance technology, and transportation materials. These areas are briefly described below.

\section{Microtechnology}

The objective of this Energy Technology Development focus is extending high-precision, microfabrication techniques, originally developed for the electronics industry, to preliminarily develop and demonstrate revolutionary thermal and chemical components and systems. Examples include compact, high efficiency, microchannel heat exchangers and fuel processors with the potential for reductions in size, weight, and capital costs compared to conventional hardware.

Microtechnology serves both Energy Technology Development initiative competency focuses: in the near-term, microtechnology-based components will prove important for operating and maintaining our existing infrastructure; and, in the longer term, microtechnology-based, energy conversion systems (e.g., heat pumps, power generators, etc.), broadly distributed to locations where their services are required, constitute the quintessential distributed energy systems. New microtechnologies could make small-scale, distributed energy processes economically attractive when compared to centralized processes normally used today.

There are numerous potential advantages which may accrue from microtechnology systems besides reduced size and weight, including 1) capital cost advantages due to economies of production, compared to the large capital investments required for large, centralized facilities; 2) employing phenomenological processes that are more powerful at the microscale than at the macroscale (such as heat transfer and mass transport); and 3) the ability to facilitate distributed systems, hence eliminating the energy transmission and distribution losses characteristic of large central systems.

Pacific Northwest National Laboratory activities are focused on design, fabrication, and testing of microtechnologyenhanced thermal and chemical components and systems. We are extending the manufacturing techniques developed for microelectronics to manufacture compact, high capacity components and are combining them into complete energy and chemical systems. We are also developing the basic system architectures for a wide variety of energy applications. For example, we have fabricated and tested the first microchannel evaporators, condensers, gas absorbers, and electromagnetic micropumps. Results show that the performance of microchannel heat and mass transfer devices are outstanding and offer the potential to radically reduce the size and weight of many energy and chemical conversion processes. We are now working on an integrated microchannel combustor/evaporator; advanced microchannel heat exchangers; compact, microtechnology-based compressors; microchannel partial oxidation reactors for hydrogen production; and microchannel power generators. Our future target is to assemble these components into prototype heat pumps, power generators, and chemical conversion systems.

\section{Transmission and Distribution Systems}

This Energy Technology Development focus seeks to develop and deploy technologies to improve the capacity, reliability, efficiency, and safety of the nation's energy transmission and distribution systems. The current focus is on electric power. Since new transmission capacity is difficult to implement, existing systems must be operated closer to their physical limits. In addition, there is a movement toward small, distributed generation and storage devices at the site of the end user (the "distributed utility"). This places strains on the stability and reliability of the nation's transmission and distribution systems that require better diagnostic systems and more effective real-time control of system operations. The distributed utility model requires close coordination of fuel delivery and generator deployment, better integration of independent renewable generators, and a better understanding of how distributed electricity generators and storage devices impact the existing electrical grid. Coordination with electric leads for enhanced grid operation is also required. All of these are, or will be, important elements in our transmission and distribution investment portfolio.

To meet the need for improved operations, the Laboratory is developing advanced analysis and simulation tools in four main areas:

- measurement-based analysis techniques for modeling and understanding power system dynamics and development of robust controls

- new theoretical, mathematical, and computational analysis techniques for model-based power system analysis, including evaluation of distributed utility options

- pulse amplitude synthesis and control technology for frequency conversion of electrical power to connect multiple distributed direct current and asynchronous alternating current generation sources to the electric grid 
- artificial intelligence-based tools for short-term load forecasting and diagnosing electrical system problems.

Development work is not being conducted in a vacuum, but rather through close coordination with the Bonneville Power Administration, the Electric Power Research Institute, and Northwest utilities, where technology is being deployed on a test basis as it is developed to make sure that it meets the needs of the utility industry.

\section{Advanced Operations and Maintenance Technology}

With the overriding need for government and industry to be more competitive comes the associated emphasis on cost control and cost-effective operating and maintenance approaches. Thus, a significant portion of our Energy Technology Development initiative is the development of technologies and systems to support good decision making in the operation and maintenance of energy systems. Our earlier work has focused on development of automated diagnostic techniques and approaches. Having developed strong capabilities in that area, we are now shifting efforts toward low-cost, higher-reliability sensor systems, another critical barrier to efficient operation of energy systems. The capabilities resulting from investment in this technical area are key to two focuses: 1) extending the life and increasing the reliability and capacity of the existing energy infrastructure, and 2) effectively operating and maintaining a large, complex network of distributed energy systems. Operations and maintenance technologies developed under the Energy Technology Development initiative will, of course, be applicable to a wide range of physical systems, not just energy systems.

Pacific Northwest National Laboratory is pursuing two approaches for reducing the cost of sensor systems, including

- a generic sensor validation approach that can be used within an operating system to detect system failure and to serve as a virtual sensor to enable continued operation of some systems until the sensor can be repaired. This approach will also provide the logical basis for minimizing sensors needed for any particular system, and for understanding the logic behind massively redundant sensor systems that are being considered as part of the microtechnology effort.

- concepts for low-cost sensors including sensors that are powered by the system they are measuring and (in later years) moving on to new approaches for remote communication with dispersed sensor suites. The initial targets here will be a low-cost self-powered device for measuring the Btu content and constituency of natural gas in pipelines, and optically powered sensor platforms for high voltage or hazardous environments.

This development work is being conducted in cooperation with other Energy Technology Development initiative elements, including the microtechnology and transmission and distribution elements.

\section{Transportation Materials}

This Energy Technology Development focus seeks to develop materials and material/component manufacturing methods that will improve the fuel efficiency of vehicles and reduce their emissions. We are also starting to focus on technologies needed for the next generation of vehicles. These technologies, once developed and demonstrated, are often widely applicable beyond the transportation arena, and this element seeks to identify alternative applications and to adapt the transportation technologies to those applications. This area largely serves the second (distributed energy system) focus, since vehicles are distributed systems, although the materials that we are developing will serve to extend the life of, and enhance the efficiency and effectiveness of, the existing energy infrastructure.

In past years, the Laboratory has focused on advanced manufacturing and coatings technologies. Superplastic forming holds the potential to reduce the weight of new generation vehicles and improve U.S. industrial manufacturing competitiveness.
The Laboratory's role has been adapting this technology from the aircraft industry, and the development of new, cheaper alloys to make the process feasible in the automobile industry. The Laboratory also developed advanced coatings techniques which can be applied for special purpose glasses, to replace paint, and to coat engine parts to provide compatibility with alcohol-based fuels. Our work in this arena continues, funded by the Office of Transportation Technologies, the Office of Energy Research, and private industry.

Our current focus is development of technology to reduce automobile emissions. Approximately 70 percent of exhaust emissions that exceed standards are generated during cold start of catalytic converter-equipped vehicles. During cold start, the converter is at insufficient temperature for catalytic reactions to occur efficiently, resulting in higher levels of hydrocarbon and carbon monoxide emissions. Although a number of approaches for reduced light-off times and improved converter operation are being investigated, there is an emerging need to have a better predictive understanding of thermal conditions and flow behavior of automotive exhaust systems. The Laboratory is focusing on the modeling of exhaust flow and heat transfer in real exhaust systems, coatings for thermal control and exhaust treatment, and thermal energy storage concepts for enhanced catalytic converter performance. Laboratory staff are also developing new manufacturing approaches that will redesign the engine cylinder to reduce crevice volume, a principal source of emissions generation.

\section{Medical Technologies and Systems}

Pacific Northwest National Laboratory and the other DOE national laboratories have requisite capabilities to make significant contributions that would reduce the cost of health care while improving quality and accessibility. The capability base within the laboratory system can support developing new imaging modalities, sensor techniques for noninvasive physiological monitoring, applications of 


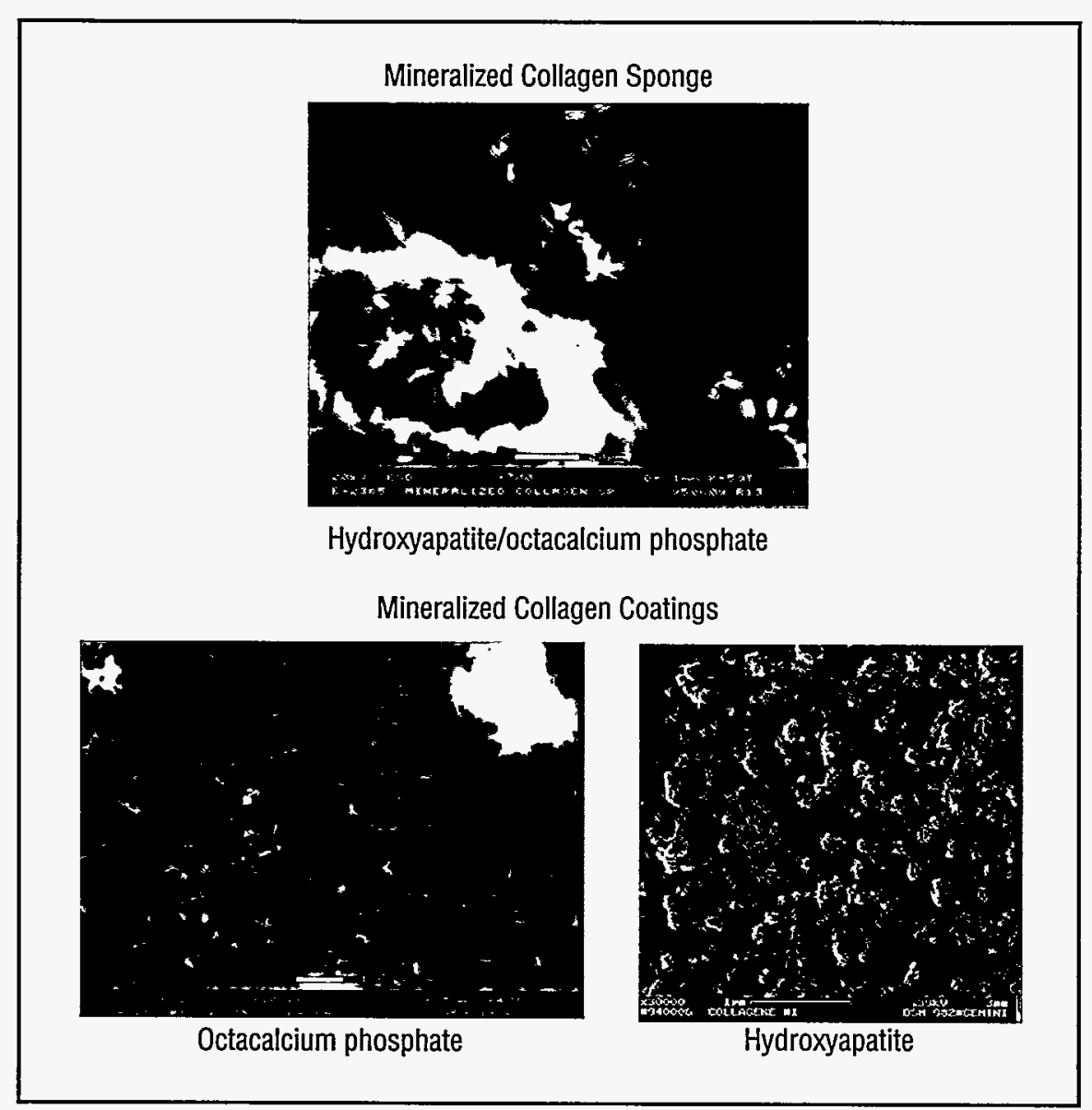

Phosphate mineralized collagen sponge and films for potential bone replacement.

powerful computational methods for network communications and analysis of large data sets, biomaterials, robotics, process improvement, policy and regulatory analysis, and mathematical modeling procedures for optimizing, integrating, and managing medical information.

As a result of ongoing needs assessments performed to identify areas where distinct Laboratory capabilities could be applied in the medical technology arena, the Laboratory is focusing on several major technological areas: 1) advanced ultrasound, 2) medical sensors, 3) medical informatics and computation, 4) targeted therapeutics, 5) medical materials, and 6) medical process improvements. Many of the technologies under consideration in each of these areas were initially developed for nonmedical applications, but are currently being adapted for use in biomedical research and clinical procedures. In several cases, these technologies have already been applied in medical practice, while in other areas research and development programs are under way to adapt new imaging, biosensing, and computational technologies for biomedical applications.

The Medical Technologies and Systems initiative is focused on integrating capabilities in fundamental life sciences with advanced technologies through directed research. The areas being investigated are

- Advanced Ultrasound - This area builds on the Laboratory's distinctive expertise in system physics, holographic techniques, integration of ultrasound with other imaging modalities, optics, and electronics to develop new reflective and transmission ultrasound modalities with improved diagnostic capabilities. Current research at the Laboratory is focused on development of twodimensional arrays, novel coupling schemes, real-time data acquisition and three-dimensional image rendering, and vector velocity measurement and display technologies.

- Medical Sensors - The Laboratory is developing new biological sensing technology to improve clinical diagnostics and treatment. Exploratory research is being conducted on the use of spectrophotometry for real-time analysis of blood chemistry. Another area of sensor development will allow doctors to diagnose disease based on analysis of a patient's breath. This capability is an extension of existing capabilities in laser and mass spectrometry. Finally, the Laboratory is developing sensor systems for real-time monitoring of radiation exposure during tumor treatment. This new sensor technology will reduce patient risk while improving the overall radiation treatment.

- Medical Informatics and Computation - The focus of this area is development of more costeffective tools for medical image visualization, diagnostic assistance, training, information processing, and telemedicine. These tools will be used by doctors to more rapidly access patient history, consult with other physicians, analyze treatment options, perform remote procedures, and improve diagnosis through the use of intelligent diagnostic agents. Through the use of technologies for distributed information management, easy-to-use human computer interfaces, and multimedia visualization, health care providers will be able to quickly find the information they need to complete accurate diagnoses and provide treatment and education. More specifically, work is currently under way to allow the doctor to view three-dimensional images and to provide the tools for accessing distributed patient records. Through the use of artificial neural networks, the Laboratory is developing tools to monitor patients' vital signs and recognize serious abnormalities. Other neural networks have been designed to analyze and diagnose 
heart disease at an early, more treatable stage, and to assist in the classification of patient headache types as an aid to the decision process for diagnosis and treatment. In the area of telemedicine, the Laboratory is developing tools for physician collaboration and consulting, as well as remote surgical assistance.

- Targeted Therapeutics - The focus of this area is on developing targeted delivery agents and systems capable of delivering therapeutic agents (drugs, toxins, radionuclides) to the site where they are most needed. Current research efforts include developing and testing techniques for quantitation and monitoring of hypoxic cells in solid tumors which will lead to the development of technology for cancer treatment. Another effort is directed toward developing a targeting/delivery agent capable of effectively binding selected $\alpha$-emitting radionuclides. Other efforts involve producing a highly specific array of substrates capable of binding enteropathic toxins and developing an aerosol delivery system for lung surfactant.

- Medical Materials - The focus of this area is the development of biocompatible materials that provide longer-lasting orthopedic implants and enhance tissue repair. Emphasis has been on the development of bioactive and porous metal coatings. Through DOE-Basic Energy Sciences support, the Laboratory has been actively involved in the research of biomimetic and advanced materials processes. These processing methods provide a unique aqueous solution deposition method for producing a wide range of uniform mineral coatings on metal, polymer, and ceramic substrates. The process involves low temperatures and is not limited by the shape or porosity of the object to be coated. The Void Metal Composite technology, developed at the Laboratory, is a process for producing a wide range of porous metal implant devices. The process enables materials to be made which are open-celled and have an interconnecting porosity and controlled density. Test implants produced via the void metal composite method have shown excellent bone ingrowth, easy fabricability, and suitable mechanical properties. The Laboratory is currently working to combine competencies in biomimetic coatings with the void metal composite technology to produce a new generation of orthopedic implant devices which can increase the longevity of surgical implants and enhance the physical well-being of patients. Medical applications of these technologies include advanced prosthetic and fixation device stabilization, integrated bone grafting for high-strength bone repair, and tissue repair after surgery.

- Medical Process Improvements Technological advances are only a part of the answer to reducing the cost of health care and improving quality and accessibility. The process by which health care is delivered, behavioral changes, and policy changes will also have significant impacts. The Laboratory believes that in order to successfully perform process improvement within medical organizations, an integrated approach is required. Current work with various organizations integrates strategic and business planning, policy analysis, stakeholder involvement, business and work process analysis, system modeling and simulation, information systems architecture, human factors analysis and modeling, material planning and inventory management, re-engineering and integration, business management, organizational design, and change management. These disciplines must be practiced in complementary fashion. Laboratory strengths focus on each of these disciplines in the context of the organization as a whole. As work is accomplished, primary value chain processes and critical support processes are monitored to ensure that tasks in all processes mesh and provide expected results without loss of quality, data, and customer satisfaction. Finally, changes are institutionalized. That is, they are not just implemented, but are securely sewn into the fabric of the organization and mesh with the continuous improvement process. This approach is applicable to organizations from the small medical clinic to the large HMOs and medical insurance organizations.

Transferring technology is an integral element of this initiative. Current efforts involve transfer to the DOE program offices, the DOD, other federal agencies, universities, medical institutions, and industry.

Programmatic support for the Medical Technologies and Systems initiative is sought through DOE offices that emphasize basic and applied research areas. Included are the Advanced Biomedical Technology Initiative, general life sciences research, structural and computational biology, microbial genome, and human genome programs in OHER, and the Biomimetics Program in Basic Energy Sciences. In addition, several of these potential opportunities are evolving with the U.S. Department of Defense/Army Surgeon General's Office, Madigan Army Medical Center, National Institutes of Health, and with industry through CRADAs and other technology transfer mechanisms. Funding requirements for the initiative are given in the table at the end of this section and are not included in the Resource Projections. 


\section{Pacific Northwest National Laboratory Initiatives}

(Budget Authorization \$ in Millions)

Initiative

1995

1996

1997

1998

19992000

2001

Advanced Processing Technology

Operating

Capital

Total Advanced Processing Technology

$\begin{array}{rrrrrrr}4.9 & 6.7 & 8.2 & 15.7 & 25.7 & 26.0 & 28.6 \\ 0.4 & 0.9 & 1.4 & 1.4 & 1.6 & 1.6 & 1.6 \\ \mathbf{5 . 3} & \mathbf{7 . 6} & \mathbf{9 . 6} & \mathbf{1 7 . 1} & \mathbf{2 7 . 3} & \mathbf{2 7 . 6} & \mathbf{3 0 . 2}\end{array}$

Energy Technology Development

Operating

Energy Efficiency and Renewable Energy

Energy Research

Laboratory Technology Transfer

Private Sector/Other

Capital

Energy Efficiency and Renewable Energy

Energy Research

Laboratory Technology Transfer

Private Sector/Other

Total Energy Technology Development

$\begin{array}{rrrrrrr}4.5 & 6.0 & 7.0 & 9.0 & 11.0 & 13.0 & 15.0 \\ 0.3 & 0.6 & 1.0 & 2.0 & 2.5 & 3.0 & 3.5 \\ 0.5 & 1.0 & 1.0 & 1.5 & 1.5 & 1.5 & 1.5 \\ 0.8 & 2.0 & 5.0 & 7.0 & 8.0 & 9.0 & 10.0 \\ & & & & & & \\ 0.0 & 0.0 & 0.0 & 0.0 & 0.0 & 0.0 & 0.0 \\ 0.0 & 0.5 & 0.5 & 0.5 & 0.5 & 0.5 & 0.5 \\ 0.0 & 0.0 & 0.0 & 0.0 & 0.0 & 0.0 & 0.0 \\ 0.0 & 0.0 & 0.0 & 0.0 & 0.0 & 0.0 & 0.0 \\ 6.1 & 10.1 & \mathbf{1 4 . 5} & \mathbf{2 0 . 0} & \mathbf{2 3 . 5} & \mathbf{2 7 . 0} & \mathbf{3 0 . 5}\end{array}$

Global Environmental Change

DOE Funding

Biological \& Environmental Research ${ }^{(2)}$

Policy, Fossil, EE

Other DOE

Work For Others

Environmental Protection Agency

Other Federal Agencies

Capital Equipment

Total Global Environmental Change

22.0

9.1

0.3

0.8

5.0

8.1

45.3

Medical Technologies and Systems

DOE

Office of Health \& Environmental Research

Basic Energy Sciences

Laboratory Technology Transfer

DOD

DHHS

Industry

Total Operating

Capital Equipment, DOE

Total Medical Technologies and Systems

$\begin{array}{rrrrrr}23.6 & 23.0 & 22.0 & 22.0 & 22.0 & 22.0 \\ 9.0 & 9.0 & 9.0 & 9.0 & 9.0 & 9.0 \\ 0.4 & 0.0 & 0.0 & 0.0 & 0.0 & 0.0 \\ & & & & & \\ 1.0 & 1.0 & 1.0 & 1.0 & 1.0 & 1.0 \\ 5.0 & 5.0 & 5.0 & 5.0 & 5.0 & 5.0 \\ 8.8 & 7.0 & 7.0 & 7.0 & 7.0 & 7.0 \\ 47.8 & 45.0 & 44.0 & 44.0 & \mathbf{4 4 . 0} & 44.0\end{array}$

$\begin{array}{rrrrrrr}0.0 & 0.0 & 0.2 & 0.5 & 0.5 & 0.5 & 0.5 \\ 0.0 & 0.2 & 0.5 & 0.5 & 0.5 & 0.5 & 0.5 \\ 0.2 & 0.3 & 0.3 & 0.5 & 0.5 & 0.5 & 0.5 \\ 0.7 & 2.0 & 3.7 & 4.5 & 6.0 & 8.0 & 9.5 \\ 0.0 & 0.2 & 0.5 & 1.5 & 3.0 & 4.2 & 5.0 \\ 0.0 & 0.5 & 1.0 & 2.0 & 4.5 & 6.0 & 9.5 \\ 0.9 & 3.2 & 6.2 & 9.5 & 15.0 & 19.7 & 25.5 \\ 0.0 & 0.0 & 0.2 & 0.5 & 0.5 & 0.5 & 0.5 \\ \mathbf{0 . 9} & \mathbf{3 . 2} & \mathbf{6 . 4} & \mathbf{1 0 . 0} & \mathbf{1 5 . 5} & \mathbf{2 0 . 2} & \mathbf{2 6 . 0}\end{array}$




\section{Pacific Northwest National Laboratory Initiatives (contd)}

(Budget Authorization \$ in Millions)

Initiative

Microbial Biotechnology

Energy Research

Environmental Management

Energy Efficiency \& Renewable Energy

Fossil Energy

Other Federal Agencies

Industry

Capital Equipment

Total Microbial Biotechnology

Complex Modeling of Physical Systems

Operating

Capital

Total Complex Modeling of Physical Systems
$1995 \quad 1996$

1997

$\begin{array}{ll}1.3 & 1.4 \\ 0.9 & 1 . \\ 0.0 & 0.1 \\ 0.0 & 0.1 \\ 0.2 & 0.5 \\ 0.5 & 0.5 \\ 0.3 & 0.5\end{array}$

3.2

0.3

0.0

0.3

0.3

0.0

1.0

0.5

0.3

1.5

1.5

1.3

3.5

0.2

1.0

1.0

0.6

9.1

1998

$1999 \quad 2000$

2001

Molecular Sciences Research

Operating

\begin{tabular}{|c|c|c|c|c|c|c|c|}
\hline Basic Energy Sciences & 6.0 & 7.0 & 8.0 & 9.0 & 9.5 & 10.0 & 10.5 \\
\hline Biological \& Environmental Research ${ }^{(b)}$ & 1.0 & 3.0 & 5.5 & 9.0 & 9.5 & 10.0 & 10.5 \\
\hline Other Energy Research & 1.0 & 1.5 & 2.0 & 3.0 & 3.1 & 3.2 & 3.4 \\
\hline $\begin{array}{l}\text { Environmental Management and Other } \\
\text { apital }\end{array}$ & 1.5 & 2.5 & 5.5 & 9.5 & 9.9 & 10.4 & 10.9 \\
\hline Basic Energy Sciences & 1.0 & 1.0 & 1.5 & 2.0 & 2.0 & 2.1 & 2.2 \\
\hline Biological \& Environmental Research ${ }^{(b)}$ & 0.3 & 1.0 & 1.5 & 2.0 & 2.1 & 2.2 & 2.3 \\
\hline Other Energy Research & 0.0 & 0.5 & 0.5 & 0.5 & 0.5 & 0.6 & 0.6 \\
\hline Environmental Management and Other & 0.2 & 0.5 & 0.5 & 0.4 & 0.4 & 0.5 & 0.6 \\
\hline Total Molecular Sciences Research & 11.0 & 17.0 & 25.0 & 35.4 & $\mathbf{3 7 . 0}$ & 39.0 & 41.0 \\
\hline otal Pacific Northwest Laboratory I & 72.1 & 90.1 & 111.1 & 138.4 & 167.7 & 191.3 & 215.2 \\
\hline
\end{tabular}

(a) Includes Atmospheric Radiation Measurement program funding, which will have significant subcontracts and is included in the resource projections.

(b) Assumes that funding for the EM Sciences Program will be managed by the Office of Energy Research. 


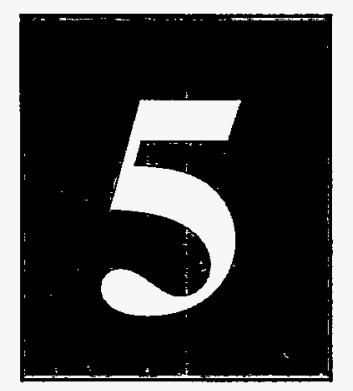

Core Business Areas

As described in Section 3, the Laboratory Strategic Plan, the Pacific Northwest National Laboratory is focusing its efforts in order to contribute to selected goals in each of the five core missions: science and technology, environmental quality, energy resources, national security, and economic productivity. Objectives have been established in each area and the Laboratory's scientific and technical programs are directed toward these objectives which are described below.

\section{Science and Technology}

Pacific Northwest National Laboratory's broad technical mission is supported by a set of critical capabilities that are based on our research facilities and the scientific and technological expertise of our staff. Integration of these capabilities across the Laboratory enables us to assemble multidisciplinary research teams that are highly effective in solving complex U.S. Department of Energy problems. Pacific Northwest National Laboratory manages its critical capabilities by integrating strategic planning, investment planning, and comprehensive assessments to focus on long-term needs. These processes guide critical decisions and activities in capability development and ensure strong relevance of the Laboratory's research to DOE missions.

The Laboratory's research activities provide new scientific knowledge and innovative concepts and technologies that contribute to the DOE's core businesses of environmental quality, energy resources, national security, and economic productivity. Science and technology activities also include education and training programs to develop and maintain a U.S. work force that is literate in science, mathematics, engineering, and technology.
Pacific Northwest National Laboratory's objectives in science and technology are to

- bring our basic scientific and technological capabilities to bear on a wide range of applied science programs to enhance the effectiveness of these programs

- advance the frontiers of environmental molecular science research to provide the knowledge needed to strengthen the Laboratory's research and development and application activities through the establishment of a state-of-the-art research user facility, the Environmental Molecular Sciences Laboratory (EMSL)

- understand the behavior of contaminants in subsurface, marine, and atmospheric environments

- enhance fundamental knowledge related to environmentally and industrially important processes, such as chemical separations and the behavior of complex fluids

- bring emerging supercomputing technologies to bear on modeling complex physical systems

- advance understanding of the health effects of chemical and radioactive substances

- improve understanding of environmentally important microbial systems

- establish and use alliances/collaborations with outside experts whose skills and capabilities complement our own strengths.

Activities for each DOE program office contributing to the Laboratory's science and technology core missions are described below. The descriptions also identify and discuss proposed new programmatic initiatives within these organizations.

\section{Environmental Molecular Sciences Laboratory}

The EMSL was first proposed as a major Pacific Northwest National Laboratory initiative in FY 1989 and was carried forward as an initiative through FY 1992 at which point it received funding from DOE. This new facility will be an essential part of the Laboratory's ability to provide the fundamental scientific understanding needed for DOE to successfully achieve its strategic environmental quality and science and technology objectives. The new laboratory will be a national collaborative research facility that will provide unique resources to the greater scientific community, enhance educational and training initiatives, and facilitate technology development and transfer among federal and state agencies and laboratories, academia, and industry.

As a collaborative research facility, the EMSL will be a national focal point for molecular science research with an emphasis on the long-term environmental management business area of DOE. As the cornerstone of Pacific Northwest National Laboratory's fundamental research portfolio, the EMSL will capitalize on today's experimental, theoretical, and computational sciences to create an expanded science base, thus enabling the development of new technologies that will not only have a positive impact on national problems but will stimulate the U.S. economy by creating new opportunities in the global technological business sector.

To achieve this goal, the Laboratory is building state-of-the-art research programs that are responsive to needs of DOE's Offices of Energy Research and Environmental Management. Special attention is given to DOE-ER-funded facilities and how they may be used to 


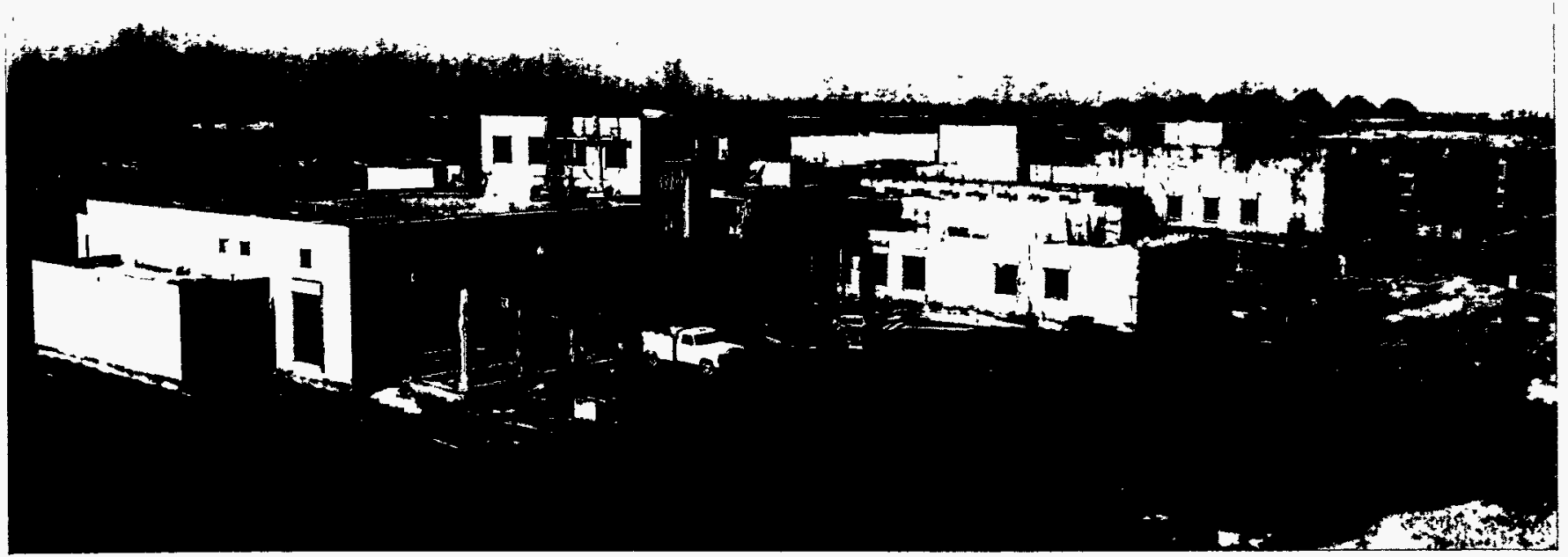

Construction of the EMSL is scheduled to be completed at the end of FY 1996.

provide research knowledge needed to help DOE-EM accomplish its environmental cleanup tasks. These efforts will be greatly enhanced by the unique facilities and equipment that will be available in the EMSL. These two elements, the EMSL capabilities and the research programs established in the EMSL to carry out Pacific Northwest National Laboratory's Molecular Sciences Research initiative, are vital to efforts to address the complex technical issues associated with DOE's environmental mission.

The EMSL provides a strong linkage among research disciplines critical to environmental remediation and waste management and establishes collaborations with members of the worldwide scientific community in these disciplines. Collaborative efforts undertaken by EMSL staff continue to increase both in number and significance. An EMSL Advisory Committee has been formed to ensure that external users have input into EMSL operations and management. Advisory committees such as the EMSL Advisory Committee will be critical to the success of the EMSL as a user facility.

In early FY 1995, a project was initiated that will lead to development of an operations plan for the EMSL. This plan will be based on the "best practices" used in industry and will be designed to meet the success criteria that have been established for the EMSL. The underlying principle guiding development of this operations plan is to balance cost effectiveness with the demands for the services associated with the primary measure for success for the new facility; that is, research of the highest value conducted by core EMSL staff and their collaborators with minimum worker risk and environmental impact. The project team visited private-sector and federal research laboratories that are recognized leaders in their fields, that have similar objectives, and that were willing to share pertinent details about their management and operations practices. Information gathered during these visits was analyzed and is being used to develop a bestpractices approach to operating the EMSL.

The DOE will spend $\$ 229.9$ million to design and develop research instruments, design and construct the building, and acquire and install the advanced instrumentation. A total of $\$ 194$ million has been provided to date to manage the project; design the facility; start construction; develop instruments and software; and develop specifications, initiate procurement actions, and start receipt for the advanced equipment that will be vital components of the laboratory. In the FY 1996 DOE budget, \$50 million in line-item funding was authorized for project management, continued equipment development and procurement activities, and construction. Prior to construction start-up, an environmental assessment for the EMSL was prepared and a finding of no significant impact was issued. Construction of the facility began in late FY 1994 and is scheduled to be completed at the end of FY 1996. Equipment systems are planned to be installed and turned over to operations in FY 1997 resulting in an overall project completion by the end of FY 1997. EMSL facilities developed under construction or programmatic support are described below.

\section{Chemical Structure and Dynamics Research Facilities}

These facilities support activities aimed at providing a fundamental, molecularlevel understanding of chemistry in a wide variety of environmentally important interfaces. The facilities will provide the means to synthesize unique molecular structures (i.e., surfaces, interfaces, and atomic and molecular clusters), to measure the structures of these model systems (including the ability to study single molecules within these structures), and to observe their chemical properties (e.g., thermodynamics, kinetics, and dynamics). This approach provides the underpinning for quantitative models 
of the complex systems found in natural and contaminated environments. The work will also develop novel sensitive and species-specific detection methods required for the wide ranging site and process characterization/analysis needs of the DOE complex.

The Surface/Interface Structure and Reactions (SISR) Facility houses surface/interface instrumentation (including 10 ultrahigh-vacuum surface science instruments) to study in detail the kinetics of molecules interacting with, and undergoing reactions at, oxide and ice surfaces and interfaces. This facility includes several molecular and ionic beam sources with the ability to grow ices doped with molecules, radicals, and ions to simulate aerosols important in atmospheric chemistry; to model mineral interface chemistry and heterogeneous catalysis; and to understand the stability of short- and long-term hazardous waste forms. Single-molecule surface structure and photochemistry studies will rely on instrumentation designed to provide direct atomic-resolution imaging of molecules on surfaces, and to follow their reactions, consisting of room temperature and low-temperature (20K) scanning tunneling microscopes with associated sample-handling and interface capabilities. The SISR Laboratory also contains six multi-wavelength laser systems and other energy sources for the initiation of chemical reactions and the state-resolved detection of chemical reaction products.

The Time-Resolved and Solution Spectroscopy Facility provides the capability for studying relaxation processes in fluids, ultrafast dynamics in biological systems, and time-resolved processes at surfaces and interfaces. It contains laser systems for generating picosecond and femtosecond pulses, including state-of-the-art femtosecond laser systems operating from the infrared through the ultraviolet, as well as picosecond systems for the study of longer time processes. These systems will be used with absorption, emission, infrared, and Raman spectrometers, with a unique collection of sample cells, for analysis of molecular dynamics and chemical reactions at environmental interfaces, such as solid mineral surfaces immersed in solutions; metal surfaces in contact with waste solutions and slurries; and surfaces important in heterogeneous atmospheric chemistry. Recently developed, near-field optical microscopes will permit the detection and spectroscopic study of single chromophores in solution, at interfaces, and in biological systems.

The Cluster Structure and Reaction Dynamics Facility contains instrumentation for studying the structure and reactivity of clusters important to understanding contaminant chemistry in solution and at mineral interfaces, and in heterogeneous atmospheric processes. It contains optical, mass, and photoelectron spectrometers forming nine independent research stations that are used in conjunction with neutral and ionic sources that provide high-flux beam currents of cold, mass-selected clusters of solvated molecules, metal alloys, metal oxides, minerals, and insulator materials for the study of cluster structures, dynamics, and reactions. The instruments include custom-designed expansion nozzles and provide highresolution infrared spectroscopic capability using semiconductor diode lasers and a Fourier-transform spectrophotometer. Long-path gas absorption cells are available for studying atmospheric chemistry and for developing sensitive remote detection and analytical techniques. Dynamics measurements will rely upon the short-pulsed laser systems described above. Cluster preparation and spectroscopic detection will utilize a number of tunable laser systems capable of providing high resolution and/or high-energy nanosecond pulses with wavelengths ranging from the infrared into the vacuum ultraviolet.

\section{Theory, Modeling, and Simulation}

Modeling molecular processes in the environment requires a fundamental understanding of molecules and their interactions in isolation (gas phase) and in liquids, on surfaces, and at interfaces (condensed phase). This is, however, one of the most challenging problems ever faced by computational molecular science:
- the molecules of importance range in size from large organic and inorganic species to biomacromolecules (e.g., chelates, dioxins, polychlorinated biphenyls, minerals, zeolites, proteins, enzymes, DNA, etc.)

- many of the molecular systems involve multiple species and multiple phases (e.g., tank waste solutions as well as heavy-metal-contaminated fluids flowing through the soil). Consideration of the scaling laws for molecular computations shows that this activity will require a level of computing and modeling capability that far exceeds (10 to 1000 times) that available with current supercomputers and molecular modeling applications.

To provide the advanced computing capability needed by the staff and collaborative users of the EMSL, a Molecular Science Computing Facility (MSCF) is being established. The MSCF will provide a robust and highly integrated computing environment, with high-speed links to external facilities within DOE, collaborating universities, and industry. The facility consists of

- The Production Computing Center, which will provide for the production computing needs of the research programs in the EMSL. The center will contain a large-scale, parallel computer system ( 256 to 512 processors) with a peak performance of 100 to 300 gigaflops (10 to 50 gigaflops sustained), 10 to 50 gigabytes of memory, and 100 to 300 gigabytes of disk storage. The High-Performance Computing Center will also contain a high-performance database and archive computer system for handling the large-scale scientific data management needs of the computational and experimental research programs in the EMSL. The archive system will have an initial capacity of $10+$ terabytes.

- The Graphics and Visualization Laboratory, which will provide highperformance production graphics and visualization facilities for the display and analysis of complex data sets from both experiments and 
simulations. This laboratory will contain four high-performance graphics workstations with high-speed connections to the computers in the High-Performance Computing Center, a video system integrated with the workstations to facilitate the display and capture of scientific data, and video editing equipment for the preparation of scientific presentations.

- The advanced computers installed in the Experimental Computing Laboratory, during EMSL operation will provide software research and development efforts with access to innovative computer systems that show promise for significantly extending the range or reducing the cost of molecular simulations.

Software development is the key if we are to benefit from the advanced computer systems installed in the MSCF. To this end, an aggressive research and development effort in molecular science software has been established as an integral component of the MSCF. The software development activities are focused in two areas:

- The High-Performance Molecular Modeling effort is focused on the development of a new generation of advanced molecular modeling software for a wide range of parallel computer systems, from clusters of workstations to the high-performance parallel computer systems to be installed in the MSCF, to the emerging teraflops class of massively parallel computers. A key aspect of this work involves the improvement and extension of the state of the art in computational chemistry algorithms. The crucial requirement for scalability of these algorithms, which determines the efficiency of an application for large numbers of processors or large problems, will not be met by conventional porting of current software to parallel computers. In many cases, the computer algorithms must be recast; in other cases, new approaches for expressing the fundamental physics will be required. The initial effort is focused on the development of software for modeling the electronic structure of molecules and solids, and for simulating the dynamics of molecules.

- The effort in the Extensible Computational Chemistry Environment is focused on the development of an extensible, integrated environment for supporting molecular research activities. Productive use of the advanced computational resources becoming available to molecular scientists requires not only a revolution in computational methods, but also a corresponding revolution in the tools for managing and analyzing computational experiments. The goal of the research and development efforts in this area is the development of an integrated, comprehensive environment for molecular modeling and simulation activities. Key components of this environment are application systems with graphical user interfaces, chemistry-specific visualization software, and tools for managing the data from molecular computations.

\section{Materials and Interfaces}

The Materials and Interfaces Research Facility will provide state-of-the-art experimental capability for research into the properties of materials and interfaces; the design, preparation, and characterization of environmentally relevant materials; and the development of advanced sensors.

- Capabilities in materials synthesis include novel methods for epitaxial and electrochemical growth and characterization of novel materials. These materials will be used in studies of model mineral surfaces, as conversion and separation materials, and in higher order chemical sensors. A particular focus will be on creation of surfaces with specific properties or types of defects. A flexible "cluster" of sputtering and vapor deposition chambers is being established to allow a wide variety of films to be deposited, including sequential deposition of different films without exposure to atmosphere.

- A spectrum of equipment for interphasial studies is included in the Materials and Interfaces Research
Facility. Probes to examine solidliquid, liquid-liquid, solid-solid interfaces include electrochemical, spectro-electrochemical, scanning probe imaging techniques, and optical methods. Also included are capabilities for determination of the structure and composition surfaces and interfaces and capabilities for examination of reactions at atmospheric pressure. The overall objectives of these capabilities are to facilitate a detailed molecular level understanding of the surface and interfacial properties of complex environmentally important systems. Consequently the range of experimental information varies from detailed analysis of model systems to characterization of complex realworld specimens. Thus the capabilities and information are designed to provide a bridge from fundamental theoretical and molecular studies to the physical chemistry of complex systems to "real-world" environmental problems.

- Planned experimental capabilities will include electron and ion based methods that primarily operate in vacuum, as well as optical, scanning probe, energetic particle, $x$-ray capabilities, and electrochemical methods that allow examination of unexposed interfaces. The materials synthesis and interphasial chemistry capabilities will be linked through a unique specimen transfer capability. The molecular beam epitaxy, chemical vapor deposition, and structural and analytical characterization capabilities will be interfaced to other EMSL equipment via passive and active transfer systems that will also allow samples to be transferred to off-site users. This aspect is particularly important to sustaining the collaborative mission of the EMSL.

- A Chemical Sensor Laboratory is being established to allow us to develop sensors to meet DOE's sensing and site characterization needs. This facility will interact with the synthesis and interphasial chemistry capabilities for the development of new sensors to work in complex environments and to meet specific 


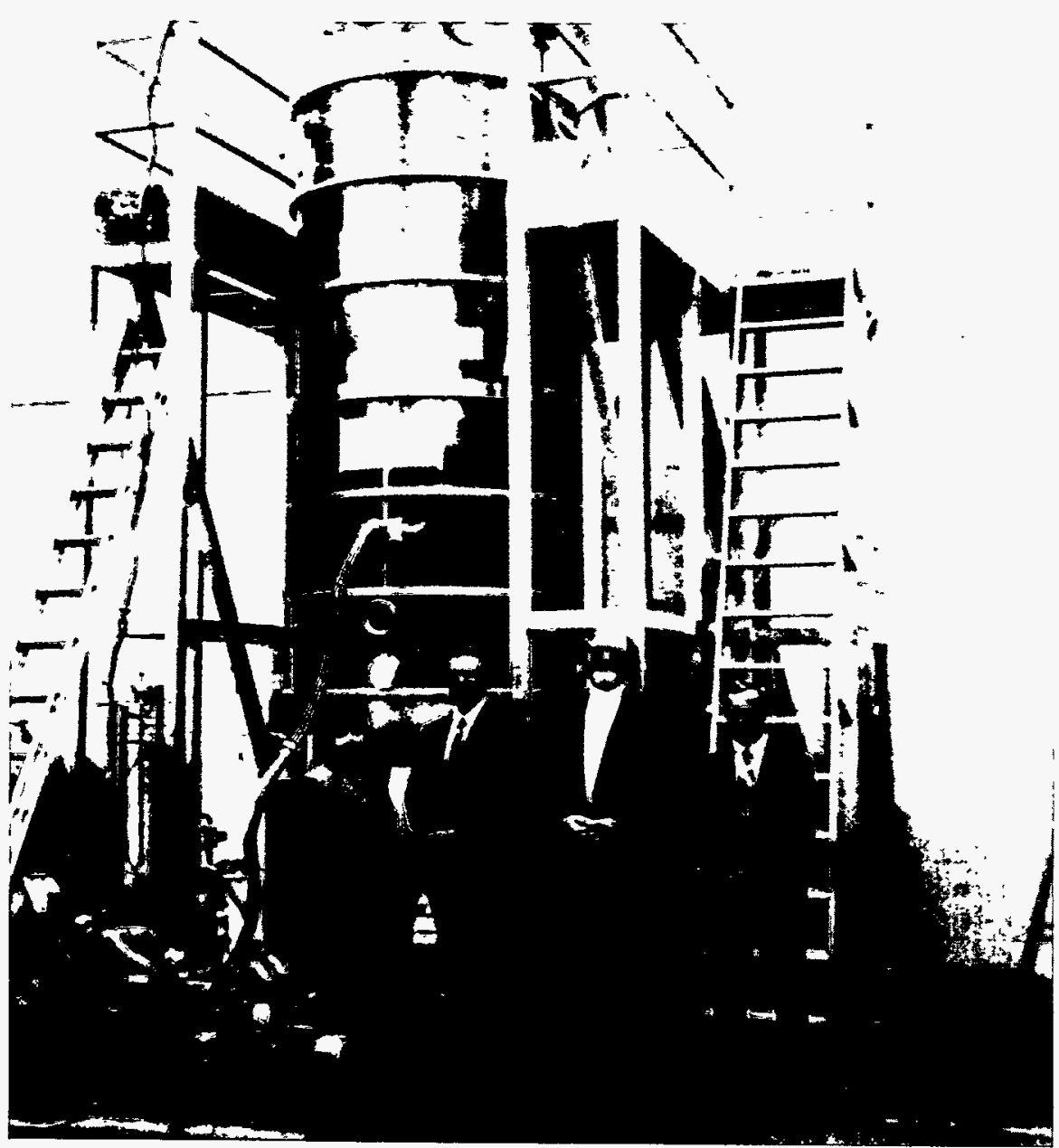

Prototype dewar for the ultrahigh frequency $(900-1000 \mathrm{MHz})$ nuclear magnetic resonance spectrometer under development for the EMSL Magnetic Resonance Facility.

DOE needs. The sensor development and testing laboratory will be a state-of-the-art resource for the evaluation of chemically selective materials, the fabrication and testing of chemical microsensors, and the development of sensor arrays and microanalytical systems. Accordingly, the Chemical Sensor Laboratory will include capabilities for the fabrication and modification of sensor devices; the application of thin films of selective materials to sensor devices; electronic equipment to characterize sensors, operate sensors, and measure sensor signals; systems to generate, dilute, and $\mathrm{mix}$ analytes for sensor testing, such as gas and vapor streams; and flow injection analysis systems for automated microscale liquid sample handling and analysis. These capabilities are supported with microcomputers and software to automate testing procedures and data collection. In addition, advanced chemometric techniques for signal processing and data analysis are an integral component of sensor and microanalytical system development.

\section{Macromolecular Structure and Dynamics}

The Magnetic Resonance and Mass Spectrometry Facilities are designed to support activities focused on 1) molecular structure of enzymes as they relate to bioremediation processes, to obtain a better understanding of their function and mechanism, especially with regard to the role of ambient inhibitors; 2) human health and environment effects as they impact risk associated with respect to environmental remediation; 3 ) waste conversion (catalysis) as it relates to the problems associated with environmental contamination; and 4) development of ultraensitive and specific detection means to provide essential in situ characterzation of the contamination site before, during, and after remediation. There are a total of 11 magnetic resonance spectrometers either operational or planned and 8 mass spectrometers when the EMSL opens. This represents a substantial asset to the DOE and to the research community at large.

- When the EMSL begins operation in early FY 1998, the Magnetic Resonance Facility will have the following in place: an ultrahigh frequency (900 to 1000 megahertz) nuclear magnetic resonance spectrometer, a 750-megahertz nuclear magnetic resonance spectrometer, and two 500-megahertz nuclear magnetic resonance spectrometers. These four spectrometers are primarily for liquids use and are completely multinuclear and multichannel devices. Further, a 500-megahertz, a 400-megahertz, and a pair of 300-megahertz, wide-bore nuclear magnetic resonance spectrometers will be available for multinuclear solid-state experiments; the ultrahigh frequency and 750-megahertz spectrometers can also be used for solid-state and microscopic imaging experiments. Finally, the Magnetic Resonance Facility will contain a multifrequency variable field-pulsed Fourier transform electron paramagnetic resonance spectrometer.

The solution phase nuclear magnetic resonance spectrometers (ultrahigh frequency and 750 megahertz) will provide unparalleled sensitivity and resolution principally for investigations in the area of bimolecular structure and dynamics for molecules of biological and/or environmental relevance. One of the 500-megahertz spectrometers will also be utilized for bimolecular structure determinations. The ultrahigh nuclear magnetic resonance spectrometer will represent the highest field nuclear magnetic resonance spectrometer in 
the world, and the magnet will be the highest persistent field magnet in the world. This high field will also be exploited for selected solid-state experiments where the high field can be used to solve important problems (e.g., the study of quadrupolar nuclides) and for imaging experiments with a subcellular resolution. The solid-state spectrometers (500 megahertz, 400 megahertz, and a pair of 300-megahertz wide-bore systems) will be used for investigations that require solid-state applications (e.g., materials chemistry and heterogeneous catalysis). The 500-megahertz system will also be used for in vivo magnetic resonance imaging of small rodents. One of the 500-megahertz liquids instruments and one of the 300-megahertz solids spectrometers will also be used to support the advanced processing applications within the EMSL. The FTEPR spectrometer will be used for those systems with unpaired electrons (e.g., metal ions in biological systems, soils, and surfaces). There are development plans to start construction of a DNP-nuclear magnetic resonance and an ultrahigh frequency pulsed electron paramagnetic resonance spectrometer in the same time frame. Finally, the magnetic resonance group will have two large computer and graphics servers for real-time support of the bimolecular structure and dynamics programs.

- The Mass Spectrometry Facility, although specializing in Fourier transform ion cyclotron resonance (FTICR) mass spectrometry, will have a broad array of spectrometers when the EMSL opens for operation (e.g., 12-, 7-, and 4.7-tesla FTICR mass spectrometers and double focusing, triple-quad, and ion trap mass spectrometers. Further, two time-of-flight [TOF] mass spectrometers will be available offering two different types of ion sources: MALDI- and ORTHO-TOF.)

The mass spectrometry laboratory will provide for a broad mass spectrometry capability. The FT-ICRMS is a one-of-a-kind instrument that will provide high sensitivity, ultrahigh resolution mass spectrometric analysis of complex mixtures and large molecular structures relevant to environmental, waste-related, bioremediation, and health-effects research. The TQMS and ITMS are commercial instruments that can both function as tandem mass spectrometry systems; they have high sample throughput, and are easily operated. The MALDI-TOF is a commercial instrument that offers extraordinary sensitivity in certain applications, in particular for biomacromolecules deposited in certain matrices on surfaces. The ORTHO-TOF is a oneof-a-kind instrument which will also provide extraordinary sensitivity for large molecules by uniquely coupling electrospray ionization capabilities to the time-of-flight mass spectrometer. Finally, the DFMS is a useful instrument offering higher energy studies with high sensitivity, which will be greatly improved by the electrospray ion source development. The DFMS is also the first stage of the planned tandem mass spectrometer.

\section{Computing and Information Sciences}

The Computing and Information Sciences program develops, maintains, and operates the advanced computing infrastructure needed for research in the EMSL, creating an integrated computing and instrumentation environment where EMSL staff, visitors, and collaborators can productively utilize computing resources to address DOE's environmental problems at the molecular level. Computing and Information Sciences research and development efforts draw from a wide range of information technologies to advance the EMSL computing environment in areas key to the EMSL computing mission. Many projects embrace collaborations with EMSL scientists, other Pacific Northwest National Laboratory researchers, universities, or industry. The Computing and Information Sciences program also provides leadership in use of emerging information technologies for EMSL collaborative research, for example, by leading the development of an Environmental Molecular Sciences Collaboratory at the Laboratory.
The EMSL Computing and Information Sciences program consists of the following elements:

- The EMSL distributed computing system encompasses all networked desktop, laboratory, and general use computers within the facility. Advances in technology are being exploited to implement a computing environment where software and computer services are available everywhere, independent of location within the facility. For example, a robust set of software applications can be used from all desktop computers, and common UNIX applications are available for all popular platforms. Software application updates and computer system management are centrally administered, ensuring that EMSL users have access to the latest capabilities. Distributed file storage, printing, authentication, and communications services round out the suite of distributed capabilities. The Distributed Computing System also contains the EMSL electronic information system, providing on-line descriptions of facilities, research program summaries, and technical reports to the World Wide Web.

- A hierarchy of data networks serves the desktop workstations, laboratory computer instrumentation, and highperformance computing systems in the EMSL. High speed computerto-computer links sustain large volume data transfers between highperformance computers, database and data archive systems, high data rate experiments, and high end graphics displays. An EMSL backbone network joins these highperformance networks to desktop and laboratory networks and work group servers. The backbone network also links EMSL to other site and national networks. Automated network monitoring and management tools, and ongoing technology evaluation efforts ensure optimal performance of EMSL networks.

- The Instrument Development Laboratory provides resources and expertise for the development and testing of data acquisition and control systems, electronics, interfaces, and 
software associated with the many instruments of the EMSL. The Instrument Development Laboratory serves as a focus for the evaluation of data acquisition and control hardware and software, as well as providing electronics design, testing, and assembly capabilities for one-of-akind and first-of-a-kind instruments. Instrument modeling resources enable optimization of instrument design and operational parameters. The Instrument Development Laboratory also provides a focal point for development of instrument hardware and software standards, and for dissemination of common solutions and acquired expertise.

- A Collaboratory is a "laboratory without walls" that spans multiple geographical areas with collaborators interacting via electronic means, and the Environmental Molecular Sciences Collaboratory will span the nation as such a resource to the molecular sciences. Research scientists are teamed with computing experts to create a new scientific computing environment for research, that provides for the integration, synthesis, and management of information from many sources and locations, along with resources that support the dynamics of research interactions among geographically dispersed scientists and research facilities.

The effort in Software Capability Development focuses on the computer science, numerical analysis, and engineering aspects of resolving questions of how molecular science applications should be structured. Software research and development efforts are advancing EMSL scientific computing capabilities to

- fully exploit the present and future massively parallel computer resources in the EMSL, through development and integration of numerical libraries and programming tools for highperformance computing

- employ computer graphics to facilitate display and analysis of complex EMSL data sets, including development of user interface and visualization tool kits for simulation or experimental data
- integrate access to scientific information databases with the operation of EMSL research applications through advanced scientific data management system designs, enabling scientists to query very large data sets and to join multiple applications for complex simulations or analyses.

\section{Environmental Dynamics and Simulation}

An integrated experimental and computational approach is required to unravel the complexities of chemical, physical, and biological processes operating in pristine and contaminated environments. An enhanced understanding of chemical processes such as mineral dissolution/precipitation, contaminant transformation and adsorption, and oxidation/reduction of natural materials will be obtained by examining the molecular mechanisms underlying these environmental phenomena. The Environmental Dynamics and Simulation Facility consists of a set of integrated laboratories that will provide spectroscopic and computational capabilities to link molecular-scale research conducted elsewhere in the EMSL to research on natural environmental systems and materials. These laboratories include the following:

\section{- Environmental Spectroscopy} Laboratory-This laboratory will provide spectroscopic capabilities for examining interfacial chemistry and reactivity of pure-phase and natural materials under ambient environmental conditions in a series of controlled-atmosphere environmental chambers. Spectroscopic systems for molecular-scale characterization of reaction mechanisms at natural mineral surfaces and in aqueous media include a fluorescence laser spectroscopy system, laser photoacoustic spectrometer, Fourier transform far-infrared/mid-infrared/ Raman spectrometer, and electron paramagnetic spectrometer. Controlled-atmosphere environmental chambers are connected directly to the spectroscopic systems, allowing the direct examination of natural environmental samples under the same ambient conditions in the field.
- Analytical Chemistry, Physical Chemistry, and Radiochemistry Laboratories-These laboratories consist of analytical systems for quantification of contaminants and reaction products, as well as thermochemical and kinetic systems for mechanistic and macroscopic characterization of contaminant-soil interactions. State-of-the-art gas/ supercritical phase/liquid chromatography with mass spectrometry will be used in conjunction with pressure-jump kinetics and calorimetry instrumentation to determine rates of complexation of environmentally significant constituents, including radionuclides, on mineral surfaces and in fluids.

- Environmental Graphics and Visualization Laboratory-This laboratory will be equipped with state-of-theart computer workstations linked to the computational infrastructure of the EMSL. Coupled geochemical reaction-transport codes developed as part of the Environmental Dynamics and Simulation Geochemical Modeling Software Library will be used to simulate complex chemical reactions coupled with flow and transport processes. The laboratory consists of several networked workstations that link into the MSCF, an integrated computing environment developed for the entire EMSL.

\section{Advanced Processing}

The EMSL Advanced Processing program is designed to link the molecular science research being performed in the EMSL to the real problems of waste cleanup by providing a pathway for developing scientific ideas and concepts into processes that address the DOE's needs, particularly at Hanford. There are three main research thrusts in the Advanced Processing program:

- conversion science-the rational design of catalysts for waste remediation, catalytic incineration, and process chemistry

- separations science-interphasial and colloidal chemistry, the rational design of separation membranes, and process design 
- materials synthesis-prepare and test materials for advanced separations and conversion processes.

Key core capabilities that crosscut these program elements include colloid chemistry, experimental and computational fluid dynamics, catalysis, chemical and surface analysis, reaction chemistry, analytical chemistry, and process control.

There are two main groupings of research equipment in the Advanced Processing program: the Spectroscopic Characterization Facility and the Process and Reaction Testing Facility.

The Spectroscopic Characterization Facility houses the following capabilities:

- Separations and Detection SystemGas chromatographic separations combined with detection and identification by mass spectrometry and infrared spectroscopy. This system will be used to study complex mixtures and matrices of inorganic and organic solids, liquids, and gases.

- Ion Analyses System-Combined instruments for the analysis of ionic species in complex mixtures and matrices, including inductively coupled plasma and atomic absorption (with graphite furnace) spectroscopy, along with an ion separation and detection capability. Speciation of anions, cations, and metal complexes will allow for the elucidation of mechanisms and the design of efficient processes.

- Thermal Process Analysis SystemThermal gravimetric analysis capability that is coupled with a differential scanning calorimeter and mass spectrometer detection to study the mechanistic details of endothermic and exothermic process reactions.

- Fourier Transform Infrared Spectrometer-A modular spectrometer that will be able to analyze solid, liquid, and gas samples. This instrument will also have the capability of interfacing with the other systems in the facility to provide spectroscopic analysis of processes.
In addition, two nuclear magnetic resonance systems in the EMSL Magnetic Resonance Facility will support spectroscopic characterization efforts. The first system, a 500-megahertz nuclear magnetic resonance spectrometer, will be used to obtain highresolution, multi-nuclear spectra of molecules and complexes in solutions. This information will help elucidate the structure and dynamics of metalligand interactions relevant to separations and conversions processes. The second system, a 300-megahertz nuclear magnetic resonance spectrometer, will be used to study the structure and dynamics of solid materials used in separation and conversion processes.

The Process and Reaction Testing Facility houses

- Chemical Reaction Test Stand System-A computer controlled automatic laboratory reactor system for the mechanistic study of chemical processes in solid, liquid, or gas phases. The system includes a reaction calorimeter to accurately measure the heat consumed or released during processes.

- Supercritical Fluid Test Stand-A two-vessel, integrated fluid processing system to study supercritical extraction and cleaning. On-line process monitoring is available through a computer interface to bench-top instrumentation, including ultraviolet-visible and infrared spectroscopy.

- Electrochemical Reaction SystemA versatile, bench-scale electrochemical system with a wide variety of electrochemical techniques available for the study of separations: salt-splitting, electrowinning, electrosynthesis, process kinetics and mechanisms, process analysis, and corrosion. The system is capable of batch or flow processing, and capabilities extend from the micro- to bench-scale and beyond.

- Membrane Reaction Development Stand-Custom designed membrane test stand for the study of processes such as reverse osmosis, ultrafiltration, and microfiltration. The test stand includes four test cells that can be operated independently or in parallel.

- Solvent Extraction Test Stand--Test stand with the capability of examining batch and continuous staged solvent extraction processes for the separation of metals and organics from aqueous and nonaqueous systems. The test stand also has the capability of long-term testing to study the effects of solvent degradation, contamination buildup, and solvent recycling.

- Tubular Reaction System-Custom designed equipment to evaluate high temperature and/or high pressure reactions in gas, liquid, and solid phases using either continuous flow or batch reactors. The system includes four continuous flow microreactors and one batch reactor. This system will be used to develop and test catalytic, thermal destruction, and thermal treatment processes.

\section{Staffing and Funding the EMSL}

The intellectual resources of the EMSL continue to grow as nationally and internationally known experts are hired to develop and lead the research programs. Research staff continue to contribute to their scientific fields by publishing papers in the technical literature, presenting research results at scientific and technical meetings, and participating in their professional organizations.

As a result of our recruiting program, the EMSL staff now totals 122, with 76 additional research and support staff matrixed from other Pacific Northwest National Laboratory organizations. In addition, 86 postdoctoral appointees are assigned to the EMSL. Two senior scientists, Dr. David Dixon and Dr. Robert Eades, joined the Laboratory in FY 1995 to lead major EMSL programs. Dr. Dixon, formerly Research Fellow/Research Leader at DuPont's Central Research and Development Department, is Associate Director of the Theory, Modeling, and 
Simulation research program, and Dr. Eades, formerly a Senior Director of Industry Marketing at Cray Research Inc., is Manager of the Molecular Science Computing Facility. Key hires anticipated in FY 1996-1997 include associate directors for the Environmental Dynamics and Simulation and the Advanced Processing programs. Additional staff to complete the EMSL's full complement of 209 permanent staff will be hired as programmatic funding support for DOE-ER/ EM coordinated research increases.

\section{Office of Energy Research}

The Pacific Northwest National Laboratory's strategy for supporting the Office of Energy Research focuses on the development of fundamental knowledge to achieve revolutionary advances in DOE's core missions. Our DOE-ER research programs form the basis for the Laboratory's contribution to the nation's science and technology leadership, and the fundamental knowledge from our research contributes strongly to DOE's other missions of environmental quality, energy resources, and national security. Our research is beneficial to DOE in determining its directions regarding scientific research, technology development, risk management (technical, social, economic) and science and technology policy that support DOE core businesses.

\section{Biological and Environmental Research (KP) $)^{(a)}$}

Pacific Northwest National Laboratory is a major participant in the DOE's Office of Health and Environmental Research. Research is increasingly focused on complex environmental and health problems that require multidisciplinary teams to address the multitude of time and spatial scales found in health and environmental research. Our health effects research ranges from the study of fundamental physical and chemical processes to the mechanisms by which hazardous physical and

(a) Budget codes are provided in parentheses next to related headings. chemical agents affect living systems. Included among the Laboratory's capabilities addressing health-related issues are cellular and molecular biology, structural and computational biology, human systems science, biotechnology, and regulatory and policy analysis. The Laboratory's environmental research is wide-ranging into the effects of natural events and human activities on the environment and on technologies to minimize environmental impacts and remediate damage caused by human activities. Our environmental research programs use stateof-the-art facilities to study subsurface science and terrestrial ecology, atmospheric chemistry (including the Laboratory's specially equipped airplane), and biological chemistry critical to understanding the transport and transformation of toxic substances in the environment. In a major research activity, the Laboratory is providing support to DOE in establishing the Atmospheric Radiation Measurement (ARM) program, a U.S. Global Environmental Change Research Program. The Laboratory is also developing, with sponsorship from OHER, the EMSL as a major research center for collaborative research related to the environment.

\section{Biological Research Biological} research is addressed through a multilevel approach incorporating studies from the molecular level to the wholeanimal level. Emphasis is placed on understanding effects at the low doses, which are characteristic of occupational and environmental exposures. This will be accomplished by gaining a quantitative understanding of fundamental mechanisms that underlie the effects of radiation and chemicals using integrated dosimetric, molecular, cellular, and experimental animal approaches. Alterations in the structure and function of genetic material are investigated both in vivo and in vitro as biological indications of the development of subsequent disease processes in living animals. Key aspects of these studies are the analysis of interactions of repair enzymes and other regulatory proteins with damaged DNA. The ultimate objective is to identify the cause of disease states induced by exposure to radiation or toxic chemicals and to predict human susceptibility to these disease states. This information will provide the scientific basis for improved risk estimation models needed to understand the health risks associated with chemically and radiologically contaminated DOE sites.

Molecular and Structural Biology Our focus is on understanding the structure-function relationships of macromolecular systems relevant to DOE programmatic needs. The resulting fundamental information can be used to identify the effects of low dose exposures and to define improved means of avoiding those exposures. Integrated experimental and theoretical capabilities for analyzing the interactions of radiation and chemicals with DNA and other macromolecules has been enhanced significantly by the new instrumentation and computing facilities being developed in the EMSL.

One long-term objective is the structural analysis of radiation- and chemical-induced DNA damage. EMSL resources enable Pacific Northwest National Laboratory's health effects research program to apply new theoretical and spectroscopic approaches to understanding mechanisms involved in mutagenesis, carcinogenesis, and developmental abnormalities through modeling three-dimensional structures of macromolecules and providing structural biology information that complements our molecular biology research. Initiatives are integrating advanced experimental techniques (such as two-dimensional nuclear magnetic resonance, electron paramagnetic resonance, and mass spectrometry) and the advanced computational and modeling capabilities in the EMSL with expanding molecular biology capabilities.

Structure-function analysis is also being applied to significant problems in microbial systems and bioremediation. Key enzymes for bioremediation are being identified through biochemical and microbial genomic approaches. The EMSL resources are ideal for understanding the mechanisms of action for these enzymes. Work is currently in progress to determine the solution structure of tetrachlorohydroquinone 
reductive dehalogenase, the first reductive dehalogenase clones. This enzyme is not only important as a dechlorinator but also serves as a model for a large class of glutathione transferase enzymes important in bioremediation.

\section{National Radiobiology Archives The} National Radiobiology Archives, developed and managed by Pacific Northwest National Laboratory, consists of a computerized information system for dose-effects data, a repository for documents and other research records, and a tissue archive, integrated by a computerized inventory system. Several DOE laboratories have stored records and selected tissues from a unique program of life-span animal studies conducted over the past five decades. As the program ends, the stored data must either be archived or discarded. Because these studies are unlikely to be repeated and represent a resource for future research, they are being preserved in the National Radiobiology Archives. The Archives will obtain as much information as possible from DOE laboratories and will focus on electronic storage and dissemination of that information. Once complete, the Archives will be reduced to a maintenance level.

Medical Internal Dosimetry The objectives of this program are to 1) provide internal dosimetry support to major medical facilities in their studies on the effectiveness of radiolabeled antibodies in the diagnosis and treatment of cancer; 2) develop improved methods for estimating radiation doses to tumors and normal tissues from incorporated radiolabeled antibodies, hormones, and growth factors; and 3) develop new alpha-particle-emitting immunoconjugates for therapy.

The Laboratory is currently conducting dosimetric assessments in support of the lymphoma and leukemia clinical trials at the Fred Hutchinson Cancer Research Center and the University of Washington in Seattle, and other clinical studies at the Virginia Mason Cancer Clinic (Seattle) and the National Institutes of Health (Bethesda, Maryland).

Pacific Northwest National Laboratory also performs internal dose assessments for animal studies and in vitro cell-irradiation experiments. Methods have been developed for assessing the localized dose distribution of nonuniformly distributed radiolabeled antibodies in solid tumors. Experiments have been undertaken with animal models to test the efficacy of using other radiolabeled compounds for tumor diagnosis and therapy.

Human Genome Research New approaches are being developed at the Laboratory with the potential for high-speed DNA sequencing based on mass spectrometry. In one approach, large single-stranded segments ( 1 to 20 kilobases) are transferred to the gas phase by an electrospray process that produces highly charged molecular ions. The ions are then trapped in an ion cyclotron resonance cell in a highmagnetic field where a single molecular ion can be trapped, isolated, and nondestructively detected with high mass measurement accuracy. The ability to trap, isolate, and measure the mass and charge properties of an individual ion for as long as hours has recently been demonstrated. The aims of the project are to develop 1) the instrumental methods necessary for such accurate mass determination, and 2) physical or chemical methods for sequentially cleaving individual nucleotide bases from one terminus of the DNA segment. An accurate and high-speed ion cyclotron resonance measurement after each step would therefore allow rapid determination of the oligonucleotide sequence for very long DNA segments.

An alternative approach to high-speed DNA sequencing is also being developed based on the analysis of mixtures of DNA fragments by electrospray ionization mass spectrometry (ESI-MS), which exploits the advantages of electrospray ionization over other ionization methods. This approach involves reducing the charge states of electrosprayed oligonucleotides by manipulating the electrospray ionization process or by subsequent gas-phase reactions. Existing mass spectrometric instrumentation is being modified to determine the maximum oligonucleotide length amenable to this approach. This approach promises to allow rapid molecular weight determination of mixture components and provide DNA sequence information analogous to the conventional electrophoresis methods. Sequencing rates on the order of 100 bases per second are projected, along with improved accuracy compared with existing methods.

Analytical Studies Pacific Northwest National Laboratory has several research programs focused on the development and enhancement of techniques for the analysis of chemical and elemental species of importance in research on environmental pollutants and human health effects. These methods generally concentrate on mass spectrometry and laser-based analytical methods. In addition to the fundamental study of analytical methods, Laboratory staff have initiated research into high-resolution spectroscopy of small molecules to increase our understanding of photoionization mechanisms and pathways. We are also proposing to establish a facility at the Laboratory for ultratrace bioanalytical research.

Mass spectroscopy research has focused on several key areas, one of which is the development of new methods for separation and detection of adducted constituents of DNA by capillary electrophoresis. These approaches are based on techniques developed at the Laboratory that combine capillary electrophoresis and mass spectrometry. The new capillary electrophoresis approach provides extremely highresolution separations of thermally labile and charged substances such as DNA and possesses several advantages over other common separation techniques. Techniques such as these are required for DNA adduct characterization because of the small sample sizes and the extremely low concentrations that must be analyzed. The capillary electrophoresis/mass spectrometry technique provides extremely sensitive detection in the range of attomolar $\left(10^{-18}\right)$ to femtomolar $\left(10^{15}\right)$ levels along with very high efficiencies. In addition, a new approach to mass spectrometric detection based on a unique orthogonal time-of-flight mass spectrometer is being developed for ultrasensitive detection. 


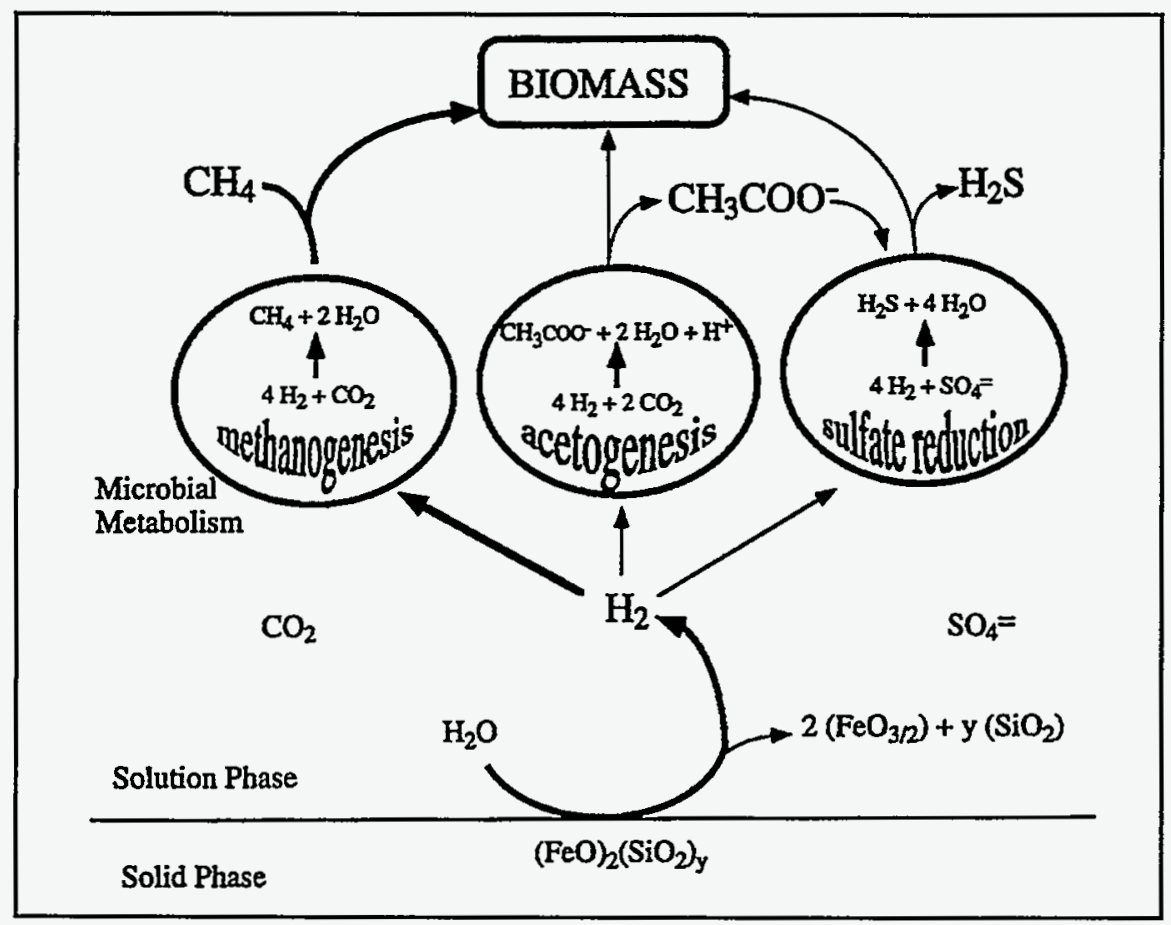

Lithoautotropic microbial ecosystems derive energy from abiotic mineral weathering and may be widespread in the subsurface. Production of $\mathrm{H}_{2}$ from $\mathrm{FeO}$-water reactions fuels the microbial community. The microbes synthesize organic carbon from dissolved $\mathrm{CO}_{2}$ and the community is independent of photosynthesis.

Several efforts related to mass spectrometry research have been concerned with the analysis of large biomolecules in complex mixtures. Of particular significance has been the progress in the development of methods for the study of biopolymers relevant to DNA damage and repair with sensitivity to allow application to small populations of cells. The Laboratory's Large-Molecule Mass Spectrometry project has focused on the use of highefficiency separations methods and the direct interfacing of these methods with mass spectrometry. Electrospray ionization, laser desorption, and other ionization methods are coupled with various separations techniques to provide new capabilities to detect, identify, and quantify compounds with very high molecular weights. This work, which has relevance to health effects research, is currently being developed as a component of the EMSL. Initial results indicate the potential for these methods to provide greatly improved sensitivity, extended molecular weight ranges, and previously unobtainable structural information. The capability has already been demonstrated for ultrasensitive analysis of macromolecules with masses of up to several megadaltons. In addition, it has been shown that noncovalently linked complexes of macromolecules can be detected under physiological conditions using ESI-MS. Powerful new methods for the characterization of proteinprotein interactions and protein inhibitors are also being developed. Other applications that are being explored include the use of mass spectrometry techniques for the analysis of interactions between damaged DNA and repair proteins and for the chemical characterization of multiply damaged sites in DNA.

\section{Subsurface Science Research Envi-} ronmental restoration will constitute a major fraction of the costs of minimizing environmental and public health risks associated with mixed wastes that have accumulated at DOE sites nationwide. Yet, there is a paucity of information and tools needed for prediction and remediation of subsurface contamination over the 20 to 30 years required to restore these sites. To help address these needs, Pacific Northwest National Laboratory research for the DOE-OHER Subsurface Science Program (SSP) builds on the Laboratory's technical strengths (geochemistry, microbiology, molecular biology, hydrology, and geology), which are applied in interdisciplinary, multiinstitutional programs focused on developing a fundamental understanding of key processes controlling contaminant behavior in subsurface systems. Unique intermediate-scale and field facilities, in conjunction with mathematical modeling, are used to examine the integrated effects of environmental processes, validate predictive models, extrapolate results to the system level, and drive new concepts for subsurface remediation. These efforts are reinforced by a major new initiative focusing on microbial biotechnology and by the vigorous collaborations with over 40 universities, other national laboratories, and industry that have been fostered by the OHER SSP and coordinated through the Laboratory's outreach activities.

A major focus of the DOE SSP investigations by the Laboratory has been on 1) co-contaminant behavior in subsurface systems and the influence of complex chemical, microbial, and physical properties and processes on contaminant transport, and 2) developing an understanding of the factors that govern the presence and activity of microorganisms in the subsurface at DOE and other sites. New emphasis is being placed on how these organisms may have adapted, evolved, and survived over millions of years under the austere environmental conditions of the deep subsurface. This research is leading to new discoveries of value for remediation and other purposes. For example, exploration of the subsurface has identified microorganisms with a unique capability for degrading a range of aromatic organic compounds under microaerophilic conditions. These organisms may offer unique potential for in situ remediation of deep contamination where oxygen is limited. Other organisms that have recently been discovered are capable of producing new products, such as dyes and antibiotics. As part of its new Laboratory 
initiative in Microbial Biotechnology, Pacific Northwest National Laboratory is extending research on the SSP to identify important microbial functions that form the basis for developing new industrial, environmental, and energy-related processes.

Examples of other SSP research under way at the Laboratory include studies to 1) examine the mechanisms underlying interfacial reactions between mixed contaminants and mineral surfaces; 2) define the factors governing the presence and activity of microorganisms in the deep subsurface and their origins and movement over geologic time; 3 ) determine the processes underlying physical partitioning of vapors, solvents, and solutes in porous media; and 4) implement advanced experimental concepts for coupling and scaling fundamental molecular, cellular, chemical, and physical processes and their interactions to understand contaminant behavior in large-scale, heterogeneous field environments.

The Environmental Science Research Center plays a special role in the SSP by exploring new concepts and approaches for understanding and restoring subsurface systems; supporting intermediate-scale and field research to scale fundamental knowledge for applications in heterogeneous subsurface environments; reaching out to the scientific community to bring new ideas, scientific disciplines, and institutions into the SSP; and promoting technology transfer to DOE sites and industry. Understanding how natural processes are coupled and scaled in heterogeneous subsurface environments helps ensure that fundamental knowledge is available for the timely resolution of long-term environmental problems faced by $\mathrm{DOE}$.

\section{Atmospheric and Climate Research} The objective of the DOE-OHER's Environmental Sciences Division is to provide scientifically defensible information on the long-term consequences of national energy use. Within the Environmental Sciences Division, global environmental change and atmospheric sciences are research components in which Pacific Northwest National Laboratory provides significant technical leadership. The Carbon Dioxide Research program is the DOE contribution to the multiagency, long-term U.S. Global Change Research Program developed by the Global Change Research Subcommittee of the Committee on Environment and Natural Resources.

Pacific Northwest National Laboratory's Global Change Program directly supports the technical objectives of the Environmental Sciences Division on environmental change. Components of the Environmental Sciences Division represented at the Laboratory include the ARM program; the Core Carbon Dioxide Research program; the CHAMMP program; the oceans research program; Global Change Integrated Assessment Research; and the quantitative links program. These programs have the common goal of improving our understanding of the physical, geological, chemical, biological, and social processes that influence the earth system so that national and international policymaking related to natural and human-induced changes in the earth system can be given a firm scientific basis. The Laboratory continues to lead the planning and organization of the ARM program and the oceans research program, as well as assisting the Environmental Sciences Division with the implementation of CHAMMP. Each program involves a number of DOE laboratories and other agencies and strong participation from the university community.

Pacific Northwest National Laboratory also plays a leading role in other longterm Environmental Sciences Division research thrusts that provide the scientific foundations on which the global change programs are based, from which energy-related air pollution assessments draw improved models, and with which safety evaluations at DOE sites reduce environmental uncertainties. This work comprises the Atmospheric Chemistry Program and the Atmospheric Studies in Complex Terrain (ASCOT) program. The Laboratory's research within the Atmospheric Chemistry Program focuses on the fate of energy-related pollutants through research on atmospheric gas-phase and heterogeneous chemistry; cloud and aerosol chemistry, physics, and dynamics; and global-scale chemical transport modeling. The Laboratory's work within the ASCOT program focuses on density-driven air circulations, turbulent mixing and dispersion in the atmospheric boundary layer, and microscale to mesoscale meteorological processes that affect air-surface exchange processes.

Global Change Research Global change research at Pacific Northwest National Laboratory focuses on studying basic geophysical processes and developing data that are critical for understanding global and regional climate change. The Laboratory is supporting the DOE goal of increasing the reliability and scientific defensibility of general circulation models (GCMs) that are used for predicting the timing and magnitude of climate change on regional and global scales by energyrelated anthropogenic emissions. Current research at the Laboratory includes the study of atmospheric radiative forcing by aerosols and clouds, the prediction of future emissions of carbon dioxide, the exchange of carbon dioxide and energy between the ocean and the atmosphere, the development of the next generation of computational resources for predicting climate change, and development of a program of research for detecting human-induced climate change.

A new focus is the use of molecular techniques to examine specific mechanisms that control ecosystem responses to environmental change. Research is directed toward understanding how energy exchange and biological (microbial, plant) processes affect mineral, water, and gaseous fluxes within arid ecosystems. These investigations are being used to determine the biotic feedback linkages that control radiatively important gaseous emissions from the environment.

The ARM program represents a major effort to improve the treatment of processes that control the redistribution of energy and water in the climate system. Recent research has revealed that radiative forcing and feedback by 


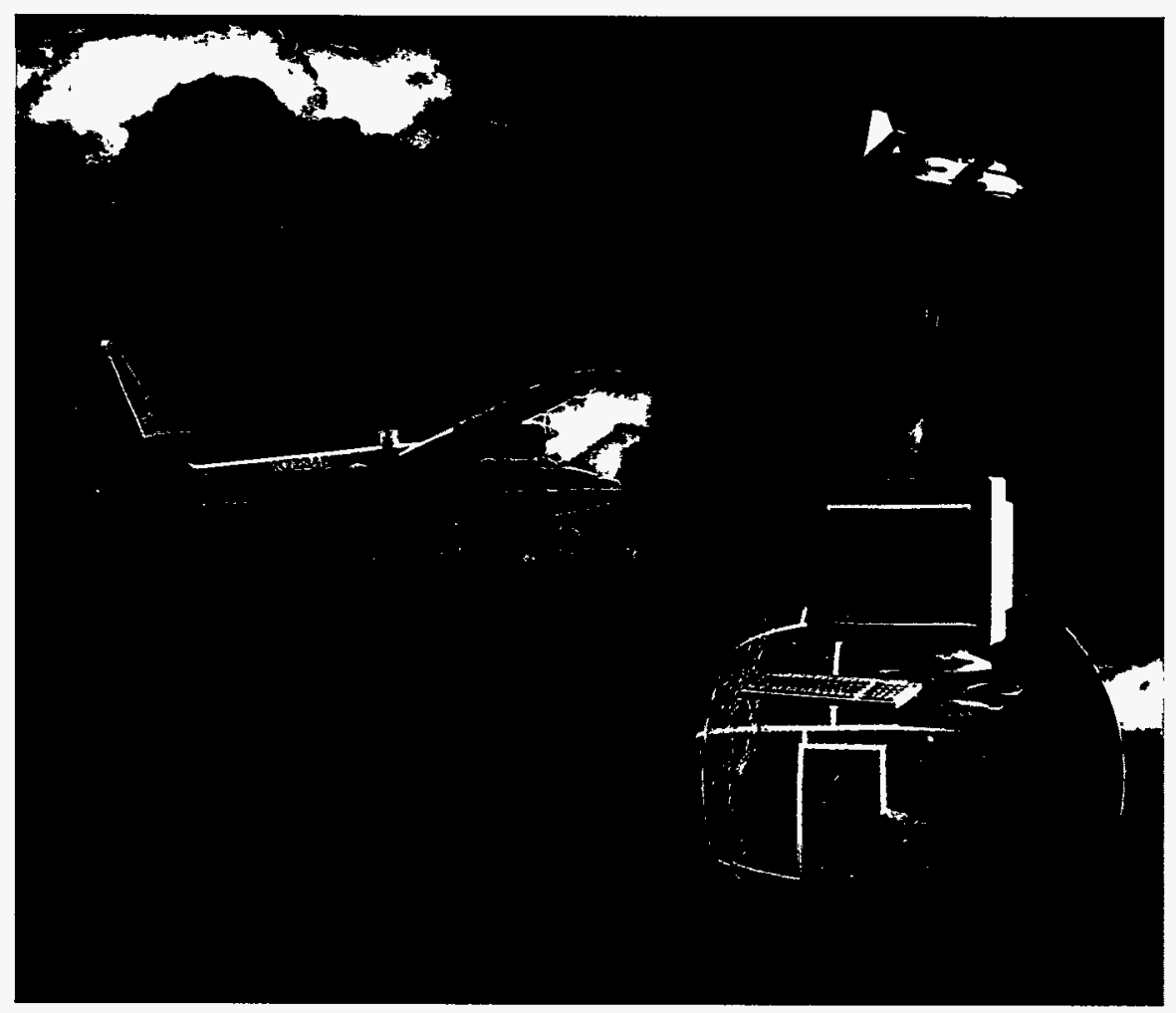

Stacked on top of one another with a layer of smooth cloud between, two planes flying over northern Oklahoma and south-central Kansas are feeding scientists new information about the way clouds absorb the sun's energy. The data gathered from this "cloud sandwich" will be used to improve computer models that predict global change.

clouds are the most important processes governing the response of the atmospheric component of the climate system to the perturbations resulting from human activity. The results of the ARM program will lead to improved general circulation models by improving the treatment of cloud radiative forcing and feedbacks and the direct and indirect effects of aerosols. ARM is focused specifically on two aspects of that improvement: 1) the treatment in general circulation models of radiative transfer under clear sky, general overcast, and broken cloud conditions; and 2) cloud formation, maintenance, and dissipation in general circulation models. Pacific Northwest National Laboratory is responsible for coordinating and integrating the field and laboratory measurement programs, modeling studies, and data analysis activities of ARM

The experimental objective of the ARM program is to characterize empirically the radiative processes in the earth's atmosphere with high spatial, temporal, western Pacific and the North Slope of Alaska. The occupation of these sites is scheduled to last 10 years. During the next few years, Pacific Northwest National Laboratory will be engaged in a series of research projects that address key ARM issues. Research is continuing on the development and testing of improved procedures for parameterizing the radiative properties of clouds, and on a study of the effect of the southern Great Plains low-level jet on cloudiness over that region. Research is also under way to improve methods by which point measurements can be extrapolated to give spatially averaged surface flux values and to improve methods for accounting in models for the efforts of subgrid-scale variability. Data from a network of multi-spectral rotating shadowband radiometers are being used along with satellite remote sensing data to determine the quantitative relationships between clouds and their effects on radiative transfer. A tethered balloon profiling system for measuring radiative profiles through the atmospheric boundary layer is also nearing completion. This latter device is designed to obtain vertical profiles of upwelling and downwelling short-wave and longwave radiation, as well as a number of other important meteorological variables. The system will operate under a range of conditions, and it will enable observations to be made in a region of the atmosphere that is not easy to monitor with current remote sensing techniques.

As the ARM program expands in the coming years to include satellites and unmanned aerospace vehicles, the efforts at the Laboratory will expand as well. A key element will be the integration of these new data streams with data from the CART sites and other atmospheric programs. The major focus for these efforts will be at the ARM Experiment Center at the Laboratory, along with the ARM data archive at Oak Ridge National Laboratory.

Convection processes, deep convection, and sea ice-convection interactions, are important transfer processes in the climate system. Oceanic deep convection is currently parameterized in ocean circulation models by 
physically unrealistic algorithms. These parameterizations limit the ability of ocean general circulation models (OGCMs) to predict the altered circulation regime that could exist in significantly different climatic conditions. A new deep-convection parameterization, developed by scientists at the Laboratory as part of the CHAMMP project, will be evaluated in a massively parallel OGCM. A surface mixedlayer parameterization, which will work together with the deep-convection parameterization, is presently under development.

Under the carbon dioxide ocean research program, high-resolution, three-dimensional process modeling is being performed by Laboratory researchers to support the development of parameterizations. This research provides a necessary link between understanding key ocean processes and ensuring that they are properly simulated in climate models. Future work on deep convection will be directed at producing empirical relationships between the forcing and deep convection. These relationships will provide a basis for an improved understanding of how variations in forcing factors affect deep convection. Experiments using the coupled ice-ocean turbulence model will provide the first detailed look at processes important in determining the ice margin extent and large-scale ice melt in the polar oceans.

Additional modeling research is being performed to examine how wind, waves, and surface heat flux affect the structure and growth of the ocean mixed layer. Turbulence in the mixed layer is directly responsible for the transport of heat, momentum, and carbon dioxide from the atmosphere into the interior ocean. Results from these detailed numerical studies will be used in the CHAMMP project aimed at improving the representation of the surface mixed layer in ocean general circulation models. As with much of the Laboratory's global change research, this work is intended to produce improved methods for parameterizing these processes in global-scale models.

Pacific Northwest National Laboratory research in the ocean sciences component of the basic carbon dioxide program is also advancing our understanding of gas exchange processes that control carbon dioxide uptake at the air/sea interface and of the exchange mechanisms between the oceanic mixed layer and waters below the thermocline. The influence of whitecaps on the rate of exchange of carbon dioxide at the ocean surface is being studied in largescale laboratory experiments and will be examined in future field studies. A principal objective is to develop empirical algorithms for translating satellite remote sensing observations into globalscale estimates of air/sea gas exchange.

More precise estimates of the magnitude of oceanic uptake of carbon dioxide from the atmosphere are needed to estimate future (10 to 100 years from present) concentrations of atmospheric carbon dioxide that are used in calculations of radiative transfer in climate models. Through the organization of a science team to guide the conduct of a global survey of carbon dioxide in the oceans, the Laboratory is assisting the DOE in coordinating its research activities with other ocean programs by assessing the results of ongoing research and establishing priorities for future research in this area.

Carbon dioxide emissions research at Pacific Northwest National Laboratory is aimed at providing the scientific basis for forecasting future emissions of carbon dioxide and other radiatively important gases. The extent of future global emissions of carbon dioxide and other radiatively important gases is one of the dominant uncertainties confronting research in global climate change. A database is being developed on traditional energy supplies and energy uses in countries within and outside the Organization for Economic Cooperation and Development, with particular focus on large countries such as China and the Former Soviet Union. The current scientific understanding of the relationship between energy production and the emissions of radiatively important gases other than carbon dioxide will be advanced. A second-generation, global greenhouse gas emissions model is being developed to provide predictions of the emissions of carbon dioxide and other gases as a function of time and nation of origin.

Based on its long-standing work on greenhouse gas emissions modeling, the Laboratory has been pioneering the development of global-scale integrated assessment models. Current efforts focus on the development of the Global Change Assessment Model (GCAM) and on the development of regionalscale offshoots of this model. The GCAM integrates an emission model that represents several important sectors of the global economy with reducedform models of agricultural production, ecosystem response, and climate change (including sea level rise) to simulate coupled economic and ecosystem response to climate change. The model can also simulate the effect of future technological innovations, as well as energy and environmental policy decisions, on the buildup of greenhouse gases, subsequent climate change, and on the global energy, industrial, and agricultural economy.

In another aspect of Pacific Northwest National Laboratory's research on the socioeconomic effects of global environmental change, the Laboratory is leading an international group of social scientists in the development of a comprehensive, state-of-the-art assessment of the ability to understand and affect social responses to global environmental change. This effort is comparable in scope to the Intergovernmental Panel on Climate Change (IPCC) assessments of the state of scientific understanding of the response of the physical climate system to increasing concentrations of greenhouse gases in the atmosphere. The social science assessment was scheduled for completion in time for the March 1995 meeting of the IPCC in Berlin.

During the past year, the Laboratory has completed the design of a program of research to determine if the expected greenhouse gas climate signal could be detected in the climate data set. The primary objectives of this research are to 1) determine if current anthropogenic perturbations to the climate system are large enough and sufficiently different from other natural climate variations to be detectable in the climate 
record, and 2) determine the extent to which current climate models are capable of simulating the broad response of the system to a gradually increasing anthropogenic forcing. This program of research, called the Early Detection of Induced Climate Trends, is the product of an international group of distinguished climate researchers. The program has been submitted to the U.S. Global Change Research Program for possible future implementation.

Atmospheric Sciences Research Atmospheric research conducted at Pacific Northwest National Laboratory is centered on two related areas, atmospheric chemistry and boundary layer meteorology. Major atmospheric chemistry studies are being conducted on the chemical processing, transport, and removal of air pollution in DOE's Atmospheric Chemistry Program. Atmospheric boundary layer research on surface energy exchanges over inhomogeneous surfaces and the interactions of thermally driven circulations induced by local terrain features with larger scale flows is being carried out as part of DOE's ASCOT program. Much of our research in both of these long-term, multilaboratory programs is carried out in collaboration with other agencies and universities.

In the 1990s, the Atmospheric Chemistry Program is moving toward research on large spatial and temporal scales to address intercontinental and global pollution concerns. Studies over the North Atlantic and North Pacific oceans and in the Arctic will investigate the oceanic and continental fates of energy-related pollutants on an international scale because of the extent to which energy production and use influence global atmospheric chemistry. Key areas of research will include processes regulating tropospheric ozone concentrations in pristine locations, the production of aerosols, the interactions of aerosols with clouds and radiation, and the exchange of material between the troposphere and stratosphere. The Laboratory's research in atmospheric chemistry will steadily expand as activities over the next several years move to increasingly complicated experimentation that is integrated with other national and international efforts. The cornerstones of the Laboratory's capabilities in its role in the Atmospheric Chemistry Program include

- aircraft measurements over the large distances and depths in the atmosphere necessary to characterize globally significant gaseous and particulate species and their chemical and physical behavior

- global chemistry modeling of detailed chemical reactions taking place on scales from single-storm processing to transformations occurring over continental and global scales of motion.

Large-scale experiments on the tropospheric chemical processing and redistribution of contaminants will rely heavily on Battelle's Gulfstream 1 turboprop aircraft. This newly designated DOE research aircraft will be available to scientists in the DOE laboratory system as well as to university researchers participating in the Atmospheric Chemistry Program and other DOE research programs such as the ARM program. Real-time measurements of trace gas concentrations were enhanced through the installation of a triple quadrupole mass spectrometer that permits analysis and real-time mapping of concentrations at parts-per-trillion levels of a broad spectrum of pollutants and naturally occurring gases. Aerosol concentration and size distributions are measured with two externally mounted particle measuring probes for two size ranges of aerosols and cloud droplets, coupled with ultrafine particle counters. Further development of the research aircraft facility will include state-of-theart real-time direct and remote sensing chemical detectors for measuring chemical fluxes in the troposphere.

Detailed descriptions of gaseous and aqueous-phase chemistry in storm models have been expanded to intercontinental and global scales. Global circulation dynamics are being coupled to advanced chemical transport models for assessing the magnitude and rate of chemical change and the global budgets of pollutants. Included in these models are the natural chemical emissions into the air from the ocean and biosphere. Future improvements to the models include better parameterizations of subgrid-scale cloud dynamics, chemistry, microphysics, and deposition. Adaptation of this modeling effort to high-performance, massively-parallel computer systems is also being carried out.

Advanced measurement and modeling capabilities will be crucial in studying the roles of anthropogenic and biogenic atmospheric chemicals in tropospheric ozone and aerosol budgets, especially over the oceans where striking differences from continental distributions prevail. Through the interaction of short- and long-wave radiative fluxes with aerosols, clouds, and trace gases over both continents and oceans, the Laboratory's research supporting the Atmospheric Chemistry Program will be closely linked with the goals of the ARM program.

ASCOT is a multilaboratory program that is being phased out by DOE. Pacific Northwest National Laboratory is a principal participant with responsibility for program direction and interlaboratory coordination. ASCOT involves scientific research in atmospheric transport and dispersion; focusing experimental, theoretical, and modeling efforts on atmospheric boundary-layer and mesoscale processes. A high priority in the ASCOT research program is to gain understanding that will permit forecasting of boundary-layer conditions hours in advance, given the transient airflow and turbulence structures that change abruptly under the influence of complex terrain and strong energy-transfer processes at the surface. To accomplish this objective, scientific emphasis in the ASCOT projects at the Laboratory is centered on the measurement and modeling of energy exchange processes as they contribute to mesoscale circulation development and evolution.

The Laboratory's ASCOT research, which previously focused on nighttime drainage and daytime transition conditions in isolated valleys, is now concentrated on the multiscale interactions of exposed valley circulations with overlying regional and synoptic airflows and on multiple valley systems. The final phase of the ASCOT 
program will be the analysis of data from recent experiments on airflows in the vicinity of Oak Ridge, Tennessee, and DOE's Rocky Flats Plant in Colorado to understand the interaction between synoptic-, regional-, and local-scale winds, which are thermally and mechanically driven.

As the ASCOT program winds down, the Laboratory's research in boundary layer meteorology and air-surface exchange processes will be redirected. Energy and mass exchanges between the earth's surface and the atmosphere are crucial to the earth's climate. Our research will emphasize their proper simulation in general circulation and climate models through efficient, accurate parameterizations. Laboratory staff will also develop a tighter coupling between atmospheric chemistry and boundary layer transport and dispersion processes. Regional and local air quality modeling used for the analysis of emissions control scenarios to meet federally mandated air quality standards will benefit from an improved capability to simulate these interactions. We will apply our capabilities to measure and characterize the structure of the boundary layer to site-specific investigations in which complex terrain exerts a significant control on the dispersion of pollutants.

The Laboratory's research and program management activities in global change and atmospheric sciences are indicative of the coordination that exists between multidisciplinary research components at the Laboratory.

\section{Basic Energy Sciences (KC)}

Research at Pacific Northwest National Laboratory supported by the Office of Basic Energy Sciences (BES) is key to the Laboratory commitment to establish a world-class science base in chemistry, materials, and computational sciences. Programs supported by DOE-BES provide strong contributions to DOE missions in basic science. Our DOE-BES programs are increasingly focused on achieving a fundamental understanding of complex, multidimensional environmental problems associated with the production and consumption of energy and the cleanup of our past environmental practices.
Chemical Sciences Pacific Northwest National Laboratory research programs funded by the Division of Chemical Sciences support the full spectrum of DOE core businesses (environment, energy, and national security) by providing a fundamental understanding of chemistry and chemical physics. Our basic research in chemistry and chemical physics increasingly is becoming focused on new ways to quantify problems and make informed decisions regarding DOE objectives in energy, environmental quality, and environmental restoration and waste management.

The Laboratory receives programmatic funding from the Fundamental Interactions branch to develop basic understandings of the kinetics, dynamics, and molecular structure which control chemical reactions. Our primary focus is in condensed phase chemistry. We conduct experimental and theoretical studies on systems ranging from model systems to multidimensional matrices in an effort to understand complex chemical systems in both the natural and manufacturing environments. This research is being performed in the interim EMSL facilities.

Research supported by the Processes and Techniques branch focuses on two areas that directly relate to DOE energy and environmental business areas. First, Laboratory staff are involved in research to further the fundamental understanding of specific physicalchemical phenomena that are key to advanced separations methods and analytical techniques. Our research focuses on the study of supercritical fluids for chemical separation and processing and the development of new analytical principles in the areas of mass spectrometry and $\mathrm{x}$-ray absorption spectroscopy for application in waste analysis and processing technologies. Second, we are investigating the fundamental physical chemistry phenomena underlying energy conversion processes for coal. This research will help us understand the energetics and other chemical mechanisms involved in conversion processes (e.g., liquefaction) that will help the nation make more cost-effective use of the energy in its coal reserves.
Molecular Theory and Modeling Developing the knowledge base needed to address the environmental restoration issues of the DOE requires a fundamental understanding of molecules and their interactions in isolation (gas phase) and in liquids, on surfaces, and at interfaces (condensed media). Research in the Molecular Theory and Modeling program involves the application of state-of-the-art methods to the solution of important environmental problems as well as the development of new theoretical methods and modeling and simulation techniques. The program is focused in four areas: cluster chemistry, solution-phase chemistry, separations chemistry, and interfacial chemistry. A common element in each of these focus areas is the need to understand molecular processes in aqueous solutions or at the interface between aqueous solutions and environmentally important materials, such as nonaqueous phase liquids, minerals, and glasses. In addition, there is a small research effort in gas-phase chemistry.

Modeling Aqueous Clusters Research in cluster chemistry is focused on the structure and properties of aqueous clusters and on the energetics and dynamics of molecular processes involving such clusters. Molecular clusters offer a unique opportunity to examine the transition from the gaseous phase to the condensed phase. In addition, to the extent that clusters model the solution phase, studies of molecular clusters provide an opportunity to ascertain the detailed effects of solvation on chemical reactivity. Finally, the intermolecular interaction potentials derived from cluster calculations lay the foundation for accurate simulations of solution processes.

$\mathrm{Ab}$ initio methodologies developed to model gas-phase molecules and molecular processes are being applied to the study of aqueous clusters. This approach is currently feasible for clusters involving six or fewer water molecules. In addition, methods used to simulate solution-phase processes are being tested for clusters against the more accurate methods. Current activities are focused on the structure and energetics of water clusters, anion-water 
clusters (H-, F-, Cl-, OH-, $\mathrm{CN}-$ ), and salt-water clusters (LiF, $\mathrm{LiCl}, \mathrm{NaF}$, $\mathrm{NaCl}$ ).

There are also activities in this area directed toward the development of improved techniques for describing the electronic structure of molecules.

Modeling Aqueous Solutions Research in solution chemistry is focused on the structure of molecules and the energetics and dynamics of molecular processes in aqueous solutions. In these studies, equilibrium properties such as average structural properties (e.g., radial distribution functions and coordination numbers) and average energetics (e.g., enthalpies and free energies of formation) are obtained from standard statistical mechanical methods such as Monte Carlo and molecular dynamics simulations. In addition, time-dependent properties (e.g., residence time of solvent molecules in the first solvation shell and correlation functions) are also obtained from molecular dynamics simulations. Molecular dynamics simulations provide a well-developed framework for describing the dynamics of molecular processes in large complex systems. This method has been particularly effective in simulating the timedependent properties within liquids.

Methods for computing the rates of activated chemical reactions in solution are also being developed. These methods are based upon well-established gas-phase theories. Because of the importance of reactions involving light atoms (such as hydrogen atom, hydride ion, or proton transfer reactions) that occur in aqueous solutions, e.g., acid and based catalyzed reaction involve proton transfer reactions, the accurate treatment of quantum mechanical effects is a focus of this work. One goal of the present work is to extend variational transition state theory methods to include solvent effects. Current activities are focused on the aqueous solvation of atomic and polyatomic ions, the energetics of ion association in water, and the effects of nonequilibrium solvation on activated chemical reactions.

Modeling Molecular Ligating Species Research on molecular ligating agents is concentrated on the structure and energetics of ion-ligand complexes and the dynamics of complex formation in aqueous solutions. The goal of this effort is to discover the factors that control the selectivity and efficiency of ligating species important in the separation of radionuclides.

Research in this effort is currently focused on the crown ethers. Three different, yet complementary approaches are being used. The first uses $a b$ initio electronic structure techniques to compute the binding energy of metal ions to crown ethers and water. The second approach is based on the application of molecular dynamics simulations with classical force fields to model the ion-crown ether association in aqueous solution. The third approach combines semiempirical electronic structure and classical force field methods in a hybrid quantum mechanical/molecular mechanical approach that is coupled with molecular dynamics to simulate aqueous solutions of ions and crown ethers. Current activities are focused on the binding of alkali ions ( $\mathrm{Li}+\mathrm{Cs}+$ ) to 18 -crown- 6 ether and the modeling of the effect of counterions on the cation-crown ether binding.

\section{Modeling Solid-Liquid Interfaces}

Research on the solid-liquid interface is focused on molecular processes at the water-mineral and glass/ceramic-water interface. The first of these efforts is a joint project with staff in the Solid State Theory and Modeling Group and is important to understanding the binding of contaminants to the soil. The second provides information on the dissolution and degradation of materials that are being proposed for longterm isolation of radionuclides.

$\mathrm{Ab}$ initio periodic Hartree-Fock theory is being used to study the structure, physical properties, and energetics of minerals and the binding of molecules to minerals. Current activities are focused on the binding and dissociation of water on magnesium oxide surfaces and the modeling of bulk and surface properties of metal oxides based on the $\mathrm{ab}$ initio calculations.

Covalently bonded materials such as glasses are being treated using a hybrid of quantum mechanical and classical force fields. Recently, a model potential that treats the bonding and nonbonding interactions separately was developed to specifically address the questions related to the geometric structure of covalently bonded liquids and amorphous materials. Current activities are focused on benchmarking these models for silicon surfaces and on extending these methods to binary mixtures.

Chemical Structure and Dynamics As a major component of the EMSL, the purposes of this program are to 1) exend the experimental characterization and theoretical description of chemical reactions to encompass the effects of condensed media and interfaces, and 2) develop a multidisciplinary capability for describing interfacial chemical processes within which the new knowledge generated can be brought to bear on complex phenomena in environmental chemistry. The success of this program will result in the achievement of a quantitative understanding of chemical reactions at interfaces. This understanding will form the basis for the development of a priori theories for predictions of macroscopic chemical behavior in condensed and heterogeneous media, adding significantly to the value of field-scale environmental models. The EMSL Chemical Structure and Dynamics program is conducting research in the following areas.

\section{Reaction Mechanisms at Solid}

Interfaces To have an impact on problems as important as soil and aquifer contaminant transport rates and persistence, the roles of atmospheric aerosols and dust in pollution and global change, and waste processing (separations, catalytic destruction, etc.), we must understand the mechanisms of interfacial reactions, and how surface structures dictate those mechanisms. Specific areas of interest include the catalyzed destruction of contaminants, complex surface kinetics and reaction mechanisms, high-temperature/highenergy reactions, and cluster chemistry in the transition region between individual molecules and extended condensed and interfacial phases.

Solutions and Solution Interfaces The Chemical Structure and Dynamics program is engaged in a significant 
effort that focuses on the dynamical aspects of aqueous solutions. No solvent is more important than water, and few have such a profoundly strong interaction with most dissolved molecules, the energetics of which often control aqueous reactions. The acidbase properties of water and of dissolved molecules are important in many groundwater, tank, and remediation reactions. To study how a proton $(\mathrm{H}+)$ transfers from one molecule to another, we are investigating the early time behavior of the $\mathrm{H}+$ motion, and how the donor and acceptor molecules begin to reshape to accommodate the $\mathrm{H}+$ transfer. Proton-transfer reactions are also being studied in water clusters and ultrafine aerosols. Another area of interest is the solid-liquid interface; for example, probing rare-earth/metal-ion complexes to explore redox mechanisms at aquatic mineral surfaces, and spectroscopic studies testing current hypotheses regarding the binding of complexes to metal oxide surfaces. Small clusters of water surrounding a molecule or ion are also being studied, for their properties are determined by the same physics that determine solvation effects in real solutions. Experimental studies of these model systems, in conjunction with detailed calculations, provide important input to theoretical models.

\section{Analytical Methods Development}

Central to the Chemical Structure and Dynamics research programs is the development of frontier analytical techniques: detecting single atoms/ molecules; controlled vaporization of complex solids; highly efficient and specific laser-induced fluorescence, Raman scattering, and ionization methods; high-sensitivity optical and mass spectroscopies; surface analysis probes; and laser-based remote sensing of trace atmospheric species. The scope of work ranges from providing specialty analytical services to the development of instruments for remote field use. Optical methods under development, including Raman, laser-induced fluorescence, and multiphoton excitation, will be combined with sampling methods ranging from molecularbeam to high-pressure techniques.
Analytical and Process Chemistry Research programs supported by the Process and Techniques branch develop fundamental understanding of complex chemical phenomena that underpin the energy and environmental restoration and waste management business areas of DOE. A long-range goal of research in the areas of chemical separation and analysis is to improve present state-ofthe-art capabilities in analytical measurement and separation of complex chemical mixtures. Our analytical chemistry research is focused on studies of the fundamental processes in laser-based vaporization/ionization and other low- and high-temperature ionization processes. Our chemical separations research examines fundamental processes in a wide variety of supercritical fluid systems. Our physical organic chemistry of coal research examines free radical chemistry reactions, which will lead to new methods for conversion of coal to liquid products.

Supercritical Fluids Research The goal of our supercritical fluids project is to provide a basis for improved molecularlevel understanding of condensedphase interactions by bridging the gap between the gaseous and liquid states and to describe the molecular interactions underlying separations in supercritical fluids. The scope of recent studies includes 1) solvation properties and their dependence on supercritical fluid density; 2) structure, formation, and stability of organized assemblies in such fluids; and 3) the basis of selective separation processes. Understanding these phenomena will lead to improved supercritical fluid separations and reaction processes and will suggest ways of improving existing processes.

Expansion of supercritical fluids research is planned to take full advantage of the novel characteristics of these systems. Much of the newly proposed work will center on the study of reverse micelles and microemulsions in supercritical and near-supercritical fluids and their uses in more complex chemical separations and in catalysis. Practical application of this work is already materializing, with the development of reverse micelle technology for the textile industry, the use of micelle systems to model formation damage in petroleum reservoirs, and the incorporation of micelles in producing nanoscale particulate materials for industry.

Laser-Based Analytical Chemistry The objective of the laser-based analytical chemistry program is to provide an improved understanding of the basic physical processes that control sample interrogation by laser irradiation. Everimproving laser technology is resulting in smaller, more monochromatic sources of laser light for analyte excitation and ionization purposes. The scope of this research includes 1) design of new approaches to laser detection methods, 2) development and understanding of the mechanisms and limitations of the techniques, and 3) demonstration of the analytical utility for developed laser analysis techniques.

New work in this area will investigate the use of a dual laser ablation system to separately and sequentially vaporize and ionize sample constituents for analytical observation. The ability to directly ablate samples using this approach may obviate lengthy sample preparation times for more traditional analysis schemes. Use of one laser to vaporize the sample and another laser to efficiently ionize the ablated material will allow optimization of both steps. Magnetic field confinement of ablated plume material is expected to dramatically enhance plume-sample fidelity and analyte sensitivity. Uitimately, application of this knowledge will result in improvements in rapid, remote, multicomponent analysis of solid samples.

Research is also conducted to develop a basic understanding of hightemperature surface chemistry in chemical analysis and to elucidate the fundamental physics and chemistry of new and novel ion sources for mass spectrometry. The work seeks to provide understanding of high-temperature surface processes which control vaporization, dissociation, and ionization so that the sensitivity, selectivity, and reliability of analytical methods can be improved. Work in this program 
addresses the basic physics and chemistry of two new sources for mass spectrometry. The first involves a gentle, specific approach to form negative molecular ions through electron capture via photon-excited molecular electronic states. The second source explores the utility of a furnace-based, combined atomization-ionization source that incorporates a plasma discharge to facilitate uniform ionization of evaporated samples. This source, and others like it, may find particular utility for direct solids analysis.

\section{Free Radical Chemistry of Coal}

Research under the free radical chemistry of coal program develops fundamental understandings of reactions of coal-related organic structure under coal liquefaction conditions and detailed structural information for coal. The primary objective of the program is to improve the base of knowledge of the structure and reactivity of coal in order to provide a predictive basis for improving coal liquefaction processes based on the fundamental understanding (i.e., kinetics, mechanisms, and thermochemical data) of the chemistry of reactive organic intermediates. Studies undertaken by the program involve a substantial effort in organosulfur chemistry and address problems of use of high-sulfur coal in liquefaction and combustion. Other studies of the free radical chemistry of coal-related molecules examine detailed molecularlevel variables for optimization of liquefaction yields and product selectivities.

Present efforts have led to new insights into the molecular structure of coal and to the principles of organic reactivity that describe the complex chemical reaction mechanisms operative in coal conversion processes. Future efforts will further enhance the state of knowledge of the structure and reactivity of coal and will focus advanced methods in laser spectroscopies, kinetics of reactions of reactive intermediates, nuclear magnetic resonance spectroscopy, and quantum chemistry to develop a predictive molecular-level, global predictive basis for coal conversion chemistry.

Materials Sciences Materials research at Pacific Northwest National Laboratory is primarily focused on 1) the development of unique synthesis techniques for producing new or improved materials in an environmentally conscious manner and 2) the reaction of materials with their environment. These activities are the scientific underpinning for many of the applied programs that address DOE mission areas and relevant technological issues. New capabilities will build on a substantial base of expertise and experience in 1) thin-film optical materials and protective coatings and 2) synthesis and characterization of advanced nanoscale composites, ceramics, and intermetallics. Current research on environmental effects on ceramic stability and on stress corrosion and corrosion fatigue of metals and ceramics is an internationally recognized area of expertise for the Laboratory. The effects of aqueous, gaseous, irradiation, and temperature environments are being evaluated.

A major emphasis of the stress corrosion cracking research area is to relate the grain boundary chemistry of materials to the initiation and growth behavior of cracks in various environments. This effort involves 1) measuring the grain boundary chemistry by Auger electron spectroscopy, 2) computer modeling the grain boundary segregation kinetics, 3) measuring the fracture mode and rate using straining electrode and fracture toughness specimens, and 4) modeling the interaction of the environment with grain boundary impurities. Crack-tip chemistry modeling is being used to identify and predict mechanisms of crack growth related to stress and corrosion. Acoustic emission monitoring is being used to evaluate crack growth processes during intergranular stress corrosion cracking. Determination of the effect of crack-tip stresses and strains on surface adsorption is also determined with in situ Auger electron spectrometer measurements as part of this effort. A new effort is focused on evaluating environmental effects on the mechanical behavior of ceramic-ceramic composites. The emphasis is on environmental interactions on the composite interfaces and how they degrade properties.

The effects of radiation on materials microstructure and/or microchemistry and water chemistry are being evaluated with respect to the mechanisms of stress corrosion in a radiation environment. Radiation phenomena affect the stress corrosion behavior of materials in 1) the light-water reactor industry, 2) fusion first-wall and blanket structures, and 3) nuclear waste canisters in deep geologic storage. This research entails a mixture of modeling, critical experiments, and detailed analysis to identify cracking mechanisms and predict material behavior. Major issues addressed include radiation effects on alloying and impurity element segregation, sensitization, crack-tip solution chemistry, corrosion processes, and deformation mechanics.

There are many shared behefits attained by the close collaboration of related materials programs in fusion energy, $\mathrm{BES}$, and fission reactor areas. Not only does the fusion materials research add value to BES' radiation effects programs, but the BES work adds value to the fusion energy materials programs. This type of leveraging is the key to maximizing resources.

Another research activity in the area of environmental effects on materials addresses surface reaction phenomena of ceramic materials in aqueous environments. This activity is focused on developing the scientific and/or mechanistic understanding that supports the containment of hazardous waste and ceramic materials durability in general. Development of structure/ property relationships for ceramic materials in a reactive environment is the focus of this research area, using systematic variations of bulk, surface, and environmental properties. A fundamental understanding of the physics and chemistry at ceramic surfaces is evolved through correlations between experimental measurement and molecular modeling methods. Primary experimental tools for molecular spectroscopy (Fourier transform infrared, Raman, x-ray photoelectron spectroscopy, and magic-angle spinning nuclear magnetic resonance) are being used to investigate initial structures and their evolution in chemically reacting environments. Model systems, electronic structure, and molecular dynamics approaches are emphasized in interpreting molecular-level phenomena. 
The combination of these techniques is used to extend the molecular information to bulk phenomena. Mechanistic models have been developed to bridge short- and long-term behavior for ceramic durability, which has particular relevance for structural ceramics, optical materials, chemical sensors, and environmental considerations.

Thin-film optical materials have long been an important area for the Laboratory. The basic research that supports this area focuses on understanding the relationship between thin-film structure and optical properties. Dielectric films (having identical chemical composition) exhibit optical and electronic property variations. These variations are influenced by film microstructure and strain heterogeneity that develop in response to deposition conditions and interfacial interactions. This research focuses on the experimental identification of chemical, physical, and structural factors that influence the optical response of thin-film materials.

The dependence of film refractive index on thickness is also considered. Several modeling approaches are used to describe the optical properties of thin films. Microstructural parameters, such as void density and crystallite size and shape, can be extracted from empirical models of the real part of the refractive index that are based on composite media approaches. Semiempirical calculations of electronic-level energies in well-defined single-crystal materials use parameterized molecular fragment and extended Hückel (tight binding) methods to determine allowed transition energies that can be related to the optical extinction coefficient. Lattice strain can be quantified from mode frequency shifts in the phonon spectrum. The relationship between localized structural perturbations induced by strain and phonon frequencies will be pursued using lattice dynamical approaches. Results will be correlated with $\mathrm{x}$-ray and Raman measurements of dielectric single crystals and films subjected to high pressures. New optical approaches for interrogating film interfaces are currently under development.

Materials synthesis research focuses on two unique routes to making new materials. The first involves using inorganic polymers as coatings on submicron ceramic powder, a process that allows high-quality nanoscale composites to be made. The second approach involves mimicking biological processes to produce better materials.

Particle interactions and packing efficiency during consolidation are influenced by the presence of polymer molecules introduced during the dispersion stage of colloidal processing. Upon adsorption, polymer-polymer repulsive interactions between adjacent particles act to prevent particle aggregation and thereby maintain a stable dispersion. Polymers also impart a lubrication property to the system and can moderate shearing forces between particles by controlling slip at the polymer-polymer or polymer-particle interface. Through these interactions, polymers can direct microstructural development of the ceramic, resulting in the evolution of a material with low void density and increased fracture toughness.

Inorganic polymers remain largely unexplored. These materials offer several advantages over organic polymers, including direct incorporation into the ceramic matrix during sintering. This project involves a systematic investigation of particle-polymer-solvent interactions using a variety of localized molecular spectroscopic probes. Particle packing efficiency and resulting microstructure following sintering are evaluated from sedimentation and other microstructural probes. Integration of spectroscopic results with microstructure and packing efficiency suggests appropriate functional group changes to the polymer to control these localized interactions.

The objective of the biomimetic research is to understand the synthesis and the underlying control mechanisms for the fabrication of ceramic/ polymer composites at low temperature using biological principles. New methods of ceramic composite production and new and/or improved ceramic composites will result. Key to reaching this goal is understanding how the "ultrastructure" of biogenic ceramic/ organic fiber composites affects the materials properties, how this structure is formed, and how we can mimic this structure and process.

The Laboratory's approach is to combine the input from many diverse fields to understand the role of ultrastructure and mechanism of mineralization. The materials properties of mollusk shell and bone have been determined and related to the ultrastructure, living bone cells have been adapted to mineralize chemically modified substrates, natural and synthetic polymers have been mineralized, and the mechanism for surface-promoted mineralization has been elucidated.

A new area of research at the Laboratory involves microstructural effects on materials deformation that includes superplastic deformation of lightweight materials. There is a new program that lends technical support to the USCAR (U.S. Council for Automotive Research) program on lightweight vehicles.

The primary growth areas for materials research are synthesis, processing optimization for waste minimization, and materials/environmental interactions. These growth areas will be pursued in conjunction with the EMSL. DOE-BES-funded material sciences programs relevant to the research areas of 1) conventional and superplastic metal forming, 2) materials joining, and 3) microstructured engineering with polymers are part of the Laboratory's participation in the DOE Center of Excellence for the Synthesis and Processing of Advanced Materials.

Engineering and Geosciences DOEBES-funded engineering and geosciences research at the Laboratory is focused on three key research areas: 1) geochemistry of mineral surfaces, 2) geoscientific data integration, and 3) radiative heat transfer mechanisms. The majority of research is focused on low-temperature geochemistry, as a result of an intentional strategic expansion of programs aimed at collaborations involving core technical capabilities in molecular science and geochemistry and the development of spectroscopic and computational capabilities within the EMSL. At DOE direction, research in aeronomy and solar-terrestrial energy transfer and interactions has been completed and 
research in geoscientific data integration using virtual reality has been significantly reduced or discontinued. A number of new theoretical and experimental investigations are under way exploring the geochemistry of the mineralaqueous interface, the structure and reactivity of minerals, and the nature of complex fluids. Research projects on the structure and reactivity of minerals include a theoretical study of the interactions of ferric oxyhydroxide surfaces with water, an experimental examination of the growth rate of different crystallographic faces of calcium carbonate using atomic force microscopy, and a project on the quantum chemistry of layered silicates. Several of these projects contribute to the research portfolio of the Environmental Dynamics and Simulation program within the EMSL. With the assistance of Laboratory Directed Research and Development funds, new concepts are being developed to relate the molecularscale knowledge gained about growth of individual crystallographic zones of carbonates to electrostatic models of sorbate interaction on such surfaces, and to assess the structure and energetics of organic ligands at aqueousmineral interfaces (e.g., alumina). A continuing effort is focused on expanding the solid earth geophysics programs, with emphasis on developing a new project that uses geologic process models as a basis for prediction of solute and colloid transport. A project was recently completed on the use of virtual environments to handle integration of large geoscientific data sets.

Researchers from the EMSL and Earth and Environmental Sciences have been funded by the DOE-BES Division of Engineering and Geosciences since FY 1993 to study the surface chemistry of carbonate minerals. The purpose of this program is to develop a fundamental, microscopic understanding of the structure and chemistry of carbonate surfaces. Our focus is on how different surface sites influence crystal growth and dissolution and on interactions with impurities and contaminants found in the environment. The project involves an interdisciplinary theoretical and experimental effort in three areas: 1) development of semiempirical models for the structure and chemistry of the calcite cleavage surfaces, 2) vacuum studies of the structure and chemistry of the cleavage surface, and 3) comparison of surfaces in vacuum with those in model geochemical environments. The most recent efforts have focused on identifying the roles of different surface sites in the dissolution of calcite in water and on the influences of adsorbates and solution impurities on calcite dissolution rates at these sites. An atomic force microscope equipped with a flow-through cell is being used to measure dissolution and growth rates in solution.

Advanced Energy Projects The Laboratory periodically submits research proposals to the Division of Advanced Energy Projects; these proposals involve research and development concepts requiring further investigation to qualify for funding by other divisions.

Future submissions to the Division of Advanced Energy Projects are expected in such areas as energy conversion, fuels processing, power source effluent remediation, or fabrication techniques. During the past year, short "white papers" describing potential advanced energy projects were submitted for review. Discussions are continuing prior to submission of a peer reviewable research proposal.

\section{Magnetic Fusion (AT)}

The Magnetic Fusion program at the Laboratory is focused on advancing first-wall and blanket technology. The long-term objective of the program is to enhance the economic and environmental attractiveness of the fusion energy option. This is accomplished by research aimed at developing reduced activation materials that provide the needed performance in the chemical and nuclear environment of a fusion reactor. The near-term objective is to meet the needs for a materials database for experimental devices such as the International Thermonuclear Experimental Reactor (ITER).

The scope of the Laboratory's work includes determining the behavior of reference candidate materials in a radiation environment, developing new materials with improved properties, developing reduced activation materials, and developing predictive capabilities through theory and modeling of radiation and chemical effects on material properties. This predictive capability is needed to account for differences in the neutron spectrum between available fission reactors and the fusion neutron environment.

The primary materials under investigation are for structural and tritium breeding applications. Research is in progress on ferritic/martensitic steels, vanadium alloys, $\mathrm{SiC} / \mathrm{SiC}$ composites, copper alloys, and beryllium. The tritium breeding performance of lithium ceramics (solid breeders) is also being evaluated.

This materials development program requires a research staff with expertise in radiation effects, facilities for handling and testing radioactive materials, and reactors for irradiating materials. The Laboratory has a dedicated team of research engineers and scientists with considerable expertise in radiation effects and excellent facilities for handling and testing radioactive materials. Reactor facilities to achieve the U.S. and international fusion goals have become a problem not only for the Laboratory but also for the U.S. and international fusion community. Radiation experiments with joint Pacific Northwest National Laboratory and Oak Ridge National Laboratory participation currently are in progress in the HFIR at Oak Ridge National Laboratory and the SM 3 reactor in Russia.

Fusion energy development continues to be a very international program. The Laboratory's international involvement includes participation in the ITER program and official international collaborations with the Japanese MONBUSHO program and with researchers in Russia. Also, the current international collaboration with MONBUSHO was scheduled to be complete in June 1995 but an extension to this collaboration has been negotiated. Funding for Phase III tegan in April 1995 and will be 30 percent more than Phase II. The structural materials effort at the Laboratory is expected to remain constant for the next 5 years while there is some uncertainty regarding the future of the solid breeder task because of the loss of the Fast Flux Test Facility (FFTF). 


\section{DOE-ER Laboratory Technology Transfer Program (KU)}

This program has produced exceptional results in developing linkages with industry and new technology that benefit the Laboratory's industry partners and contribute to the achievement of its core missions. The majority of the technologies supported by the program at the Laboratory have linkages with Energy Research-funded activities. Despite these positive results, the future of this program is in doubt because of Congressional budget reductions. Our current plan is to support the program's survival through a continued commitment to producing positive results and further strengthening of the linkage of the program to achieving the scientific and technical missions of the Laboratory and DOE. Because of the strong linkage of the Laboratory Technology Transfer Program to DOE's economic productivity business area, a detailed description of the program is presented later in the Economic Productivity section of the Institutional Plan.

\section{Nuclear Physics (KB/GB)}

Laboratory staff are participating in discussions and planning for a proposed National Radioactive Ion Beam Accelerator project. The proposed radioactive ion beam accelerator (to be known as the IsoSpin Laboratory-ISL) will be used to study nuclear reactions and properties of nuclides at extreme values for the ratio of neutrons to protons in the nucleus. We are also supporting the Canadian ISAC proposal to build an exotic beam accelerator facility at TRIUMF.

In the near future, we expect to submit proposals to the DOE Office of Nuclear Physics Research to support basic research on exotic nuclides.

\section{High Energy Physics (KA)}

The measurement of the double-beta decay of germanium-76 is considered by most physicists to be the experiment having the highest potential for setting the world standards on lepton nonconservation, neutrino mass, and grand unification. We designed and built the most sensitive experiment in the world for making this measurement.

\section{FLC Award for Excellence in Technology Transfer}

The Ultrasonic Microstructural Analyzer system uses high-frequency ultrasonic waves to analyze the subsurface microstructure of metal components. Automobile manufacturers are evaluating the unit for improved process control when hardening steel components such as axles and cam shafts.

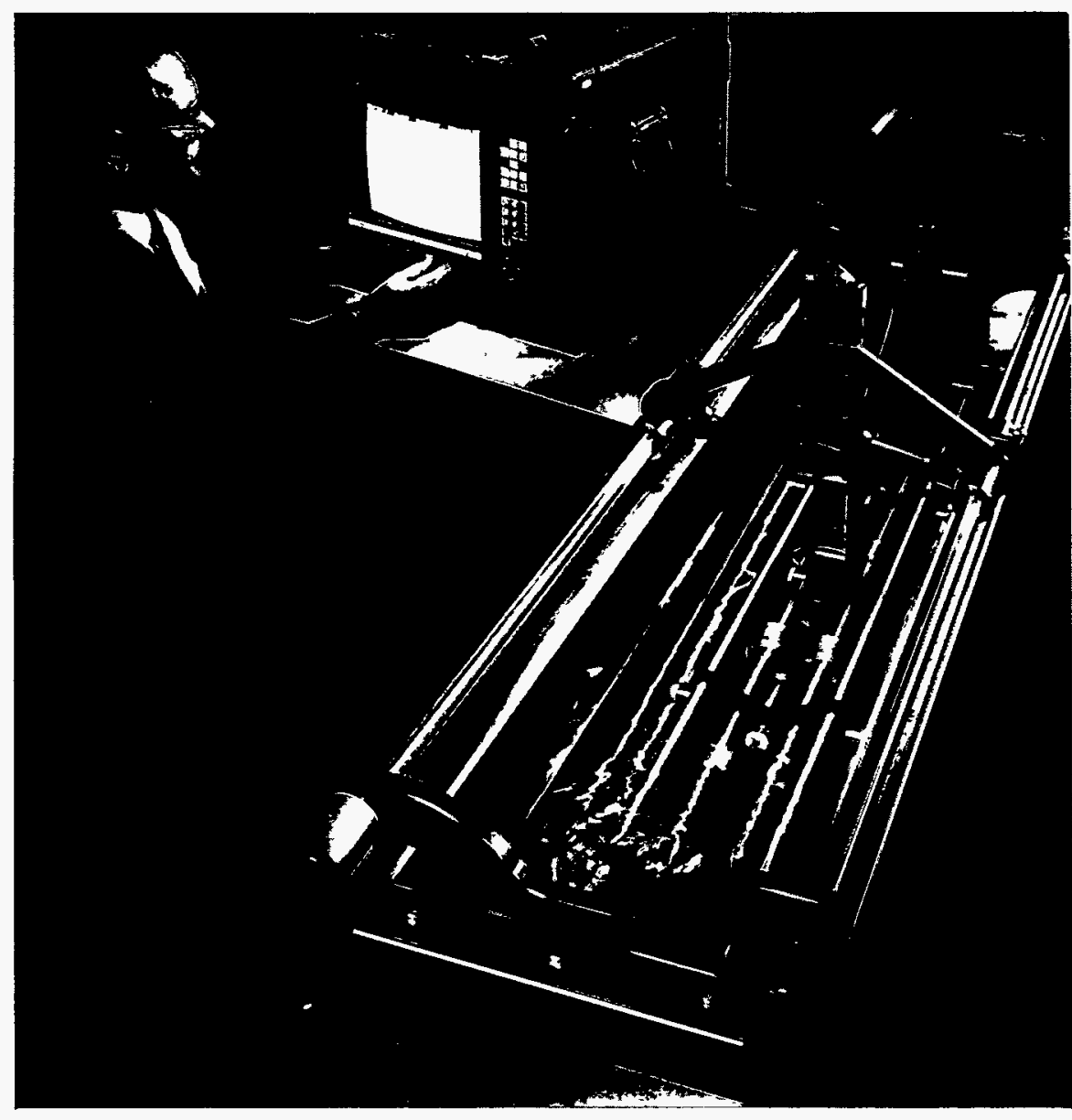

In Phase I, three 1-kilogram detectors were fabricated from 5 kilograms of enriched germanium-76 supplied to us by the Former Soviet Union. We made the first confirmed measurement of the two-neutrino double-beta decay of germanium-76 and the first measurement of the double-beta decay of molybdenum-100 to the first excited $0+$ state of ruthenium- 100 .

An international consortium, the International Germanium Experiment (IGEX), has been formed including Pacific Northwest National Laboratory; the universities of Minnesota, South Carolina, and Zaragoza, Spain; the Institute for Theoretical and
Experimental Physics in Moscow; the Yerevan Physical Institute; and the Russian Academy of Sciences' Institute for Nuclear Research, to produce several more germanium detectors enriched to 86 percent in germanium-76. The Former Soviet Union delivered 20 kilgrams of this enriched germanium (worth $\$ 30$ million) to the United States for fabrication into very large ultralow-background detectors by the Laboratory. The first two of these detectors, weighing more than 2 kilograms each, have been installed in the Homestake Mine in Lead, South Dakota. The third detector will be installed in a newly completed underground laboratory in Canfranc, 
Spain. A total of at least five such large detectors are planned within the next few years. The goal of the Laboratory and IGEX is to measure, or place meaningful limits on, the zeroneutrino double-beta decay mode of germanium-76 for resolution of Grand Unified Theories.

A collaboration including Pacific Northwest National Laboratory and the Universities of South Carolina; Zaragoza, Spain; and Buenos Aires, Argentina, has established a "cosmion" experiment in the southern hemisphere. The design takes advantage of the maximum variation in the thickness of earth available in the southern hemisphere for an experiment observing diurnal modulation of interactions engendered by cold dark matter candidates as the earth passes through the galactic halo. The detector for this experiment was fabricated by the Laboratory and Princeton Gamma Tech and installed in an iron mine in Sierra Grande, Argentina. The goal of the experiment is to elucidate potential candidates for the missing (nonluminous) mass of the universe.

\section{Science, Mathematics, Engineering, and Technology Education}

Along the educational pipeline, from elementary school through postdoctoral studies, education programs at the Pacific Northwest National Laboratory aim to increase the quantity, quality, and diversity of students preparing to participate as scientific and technical professionals in areas related to DOE and Laboratory missions; to assist in the reform of the education system so that all citizens are educated to understand science and technology and the need for high quality science, mathematics, engineering, and technology education; and to support DOE and the Laboratory's missions in energy, the environment, and economic competitiveness through interactions with universities.

The Laboratory's educational activities include an array of educational programs and interactions for students and faculty at the precollege, 2-year institution, and university levels. The programs are based on the premise that the national laboratory system and the core competencies and unique capabilities of the Laboratory provide a rich technical and human resource for enhancing the education of students and improving the education system.

The Laboratory's education programs support four overarching goals:

- supporting promising students in a successful transition from school to work

- developing the diversity of students in the education and work force pipeline

- promoting systemic reform of mathematics, science, and technology education

- building strong Laboratoryuniversity partnerships.

\section{Supporting the School-to-Work Transition}

About 20 percent of the nation's students drop out before they finish high school. Of those who graduate, less than 40 percent make the transition from high school to college. Overall, less than 25 percent remain to complete a 4-year degree. Currently, 75 percent of students in this state and nation attempt to enter the work force directly from high school or following 1 to 2 years of college. Many are not successful in their transition from school to work, particularly in work areas requiring knowledge and skill in science, mathematics, and technology. They lack the basic academic and entry-level occupational skills necessary to succeed.

The President's School-to-Work Opportunities Act of 1993, passed by Congress and signed by the President, is a critical component of the nation's response to the challenge to prepare every American for employment and productive citizenship. The Laboratory's education manager serves on Washington State's Governor's Council on School to Work Transition, which resulted from the Act. The Act calls for multiple, flexible educational and training pathways for precollege and postsecondary students. Laboratory education programs align with the national and state school-to-work agenda by proposing to establish a Technology Apprenticeship Program (TAP), providing paid internships at the Laboratory connected to a program of instruction in the schools. This program will provide relevance and practical application to academic studies and increase students' ability to transition successfully to the world of work.

Hands-on, performance based, structured training and mentoring at the work site for students participating in the Technology Apprenticeship Program will support the establishment of a program of instruction in the schools, based on career majors, that meets high academic and occupational standards and reflects the technology and training needs of the Laboratory work assignment. The Laboratory will assist in setting up connecting activities that coordinate school-based learning with work-based learning.

The Technology Apprenticeship Program will also assist schools with curricula and instruction that reflect the integration of the disciplines in the scientific and technical workplace. Opportunities will be provided for teachers and faculty to develop content knowledge and instructional strategies that successfully integrate the teaching of technology into the mathematics and science curricula. Such interdisciplinary curricula will reach not only the majority of students who never complete college, but will also enrich programs designed for all students including those who pursue careers in science and engineering.

Foundation at the K-14 Level A foundation exists at the Laboratory for supporting the school-to-work transition. At the precollege level, the Laboratory supports career awareness presentations and workshops for students. At the community college level, the Laboratory assisted in the development of the hazardous materials technician training program at the local community college which builds on curricula at the high school level. During FY 1996, the Laboratory continues to participate in the development of the Tech Prep Consortium which includes local 
academic partners at the high school and community college levels dedicated to providing continuity in specific science and technology curricula between the high school and community college. OPTIONS scholarships and internships provided to multicultural and nontraditional community college students also support the school-to-work transition.

\section{Undergraduate and Graduate Links to} the Laboratory Workplace At the university level, the school-to-work transition is supported by existing programs for students. Outstanding undergraduate students spend time at the Laboratory during the fall or spring academic semester through the DOE Science and Engineering Research Semester (SERS) program. SERS provides students the opportunity to work in a hands-on research environment under the guidance of Laboratory scientists and engineers. During FY 1996, 68 students participated in SERS.

Through AWU NW (Associated Western Universities, Inc., Northwest Division), the Laboratory hosts college and university undergraduate and graduate students for high quality summer education and training opportunities at the Laboratory as part of the DOE Laboratory Cooperative (Lab Coop) program. Laboratory research staff strongly support the goal of the Lab Coop to enhance the training of outstanding university students. During FY 1995, more than 254 undergraduate appointees participated in the program. The Lab Coop program is expected to continue healthy growth in the coming years.

In FY 1997 a proposal to the National Science Foundation will be developed to provide undergraduate faculty training at regional universities using the Environmental Molecular Sciences Laboratory "Collaboratory" concept. A faculty enhancement course in one or more technical fields will be developed to assist faculty with curriculum development.

At the graduate level, the Laboratory supports Energy Research Fellowships for highly qualified students from nine regional universities. The fellowship allows students to conduct course work on campus during the academic year and spend 3 months each year conducting research onsite at the Laboratory. During FY 1996, 6 graduate fellowships were awarded to $\mathrm{Ph} . \mathrm{D}$. candidates studying a variety of research topics.

The Laboratory hosts recipients of DOE's many graduate fellowships including Industrìal Hygiene, Operational Health Physics, Nuclear Engineering, Environmental Restoration and Waste Management, and Fusion Energy Technology. Graduate students spend 3 months conducting research at the Laboratory and complete their academic requirements at the university.

\section{Developing Diversity in the Pipeline}

The need for increasing the diversity of students in the pipeline is twofold. Jobs requiring scientific and technical knowledge and skill are increasing. At the same time, traditional sources of scientific and technical professionals are decreasing in relation to the populations of those who traditionally do not enter scientific and technical fields. Demographic trends indicate that 85 percent of new workers entering the work force between now and the year 2000 will be those from nontraditional groups such as women and ethnic minorities. Therefore, unless an increased number of individuals from nontraditional groups are attracted into scientific and technical fields, the future need for qualified scientific and technical professionals, particularly in selected disciplines, may exceed the available supply.

Equally compelling is the need to diversify the work force because diversity contributes to excellence in our work. The distinctive perspectives found in the complex richness of a diverse work force engender creativity and innovation important to solving complex problems related to energy, the environment, and economic competitiveness.

To develop diversity for a future work force, education activities at the Laboratory that target the traditionally underrepresented will form a seamless pipeline from the precollege through the university level. Resources will be applied to ensure that segments of the pipeline currently weakest will be strengthened to sustain promising students at every stage of their progress. Strategies will be employed to ensure that students, once selected to participate, are supported from year to year as they progress through the pipeline and make the transition from precollege to college and university to employment.

Linkages will be strengthened between those at the Laboratory who conduct education programs and those involved in recruiting and hiring new employees. Additional partnerships will be established among those within business lines who are seeking new employees to engage them in drawing from the pool of students in whom DOE and the Laboratory have invested by way of laboratory research and training appointments.

Following are descriptions of education programs dedicated to increasing diversity in the education and work force pipeline.

\section{Middle and High Schools and}

Two-Year Institutions At the critical middle school level, the Laboratory's OPTIONS Program expanded in 1994 to additional middle schools in Washington and Oregon having high percentages of traditionally underrepresented ethnic minorities. OPTIONS provides direct interaction between Laboratory staff and the students and teachers in the schools to stimulate students' interest in science and enhance teachers' curricular resources and teaching strategies.

Begun in 1979 and once exclusively a high school level program, the Student Research Apprenticeship Program, an 8-week summer program for traditionally underrepresented students in eastern Washington and Oregon, has been extended through the college and university years to provide continuity from grade to grade beyond the high school level of the entering students. This program is a cornerstone of developing diversity in the Laboratory's education and work force pipeline. It addresses a major weakness in the pipeline for minority students, that is, the transition from high school to higher education. 


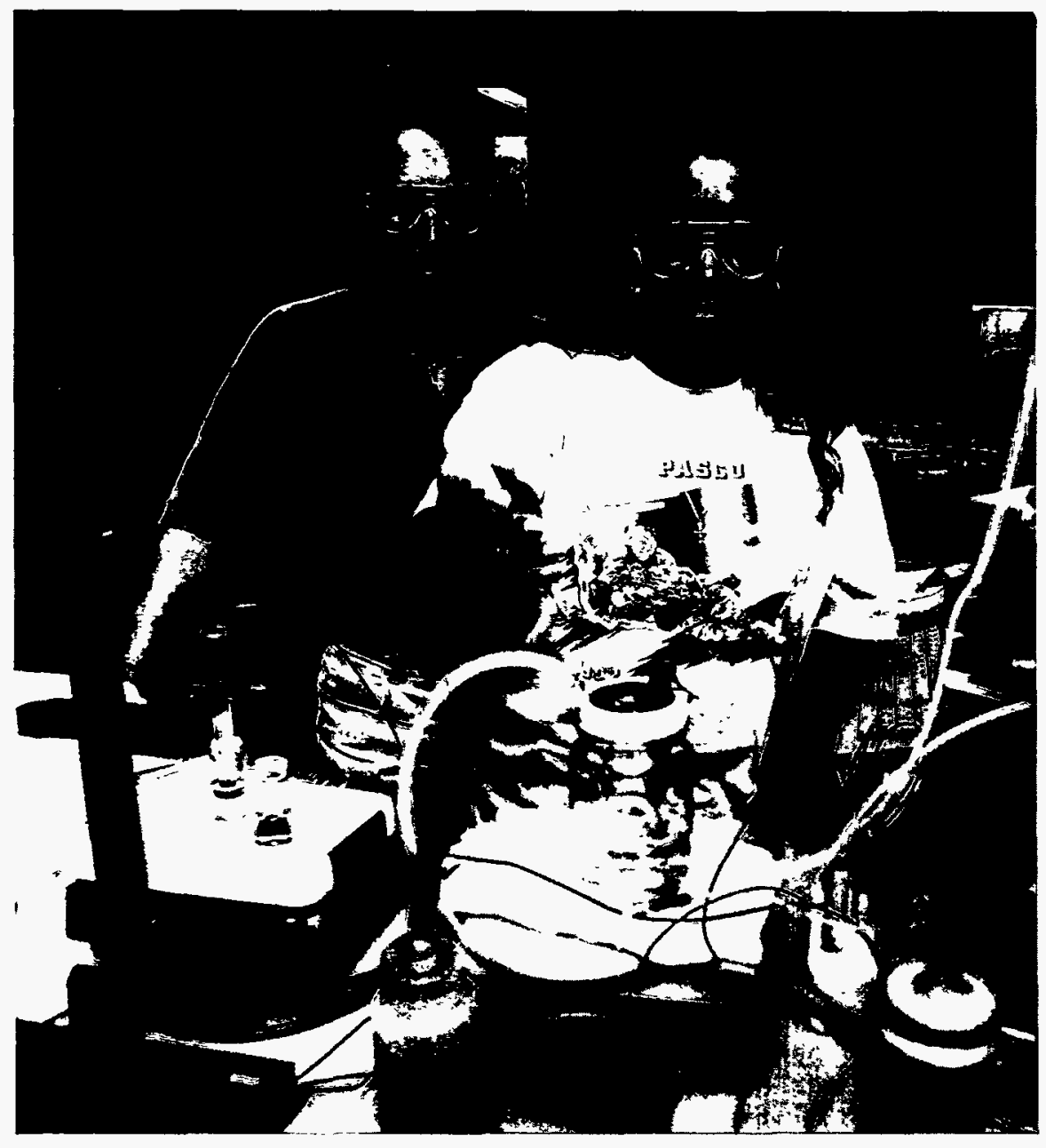

This Student Research Apprenticeship Program participant is shown here analyzing the process of biomineralization and extrapolating the process to bioactive coatings and collective composites.

At the middle and high school levels, incentives and opportunities continue to be provided for students who participate in MESA (Mathematics, Engineering and Science Achievement), a statewide program to encourage minority students to prepare for careers in science and engineering. Laboratory staff continue to participate on the advisory boards of the local MESA center and as Vice Chair of the MESA State Board of Directors. This Laboratory investment is leveraged by Battelle, which recently awarded financial support to assist MESA in ensuring that the legislated education reform in Washington State takes into account the needs of a diverse population of students.

The Columbia Basin College OPTIONS Scholars Program for Multi-Cultural, Nontraditional Students, an extension of the middle school OPTIONS program, forms a 2-year college segment of the pipeline. The program provides awards to multi-cultural, nontraditional students each year for academic tuition and fees and a summer internship at the Laboratory. Students who participate are from ethic minority groups, or are among those who are returning to school after having dropped out of the mainstream of students whose academic pathway is direct and continuous.

Higher Education Under a Memorandum of Understanding, the Laboratory continues to support Heritage College, adjacent to the Yakama Indian Reservation, whose student body comprises mainly nontraditional or place bound students from the surrounding Native American and Hispanic American communities. As a member institution of the Hanford Environmental Science and Engineering Consortium (HESEC) (described below), Heritage science and education faculty in collaboration with the Laboratory are developing a basis for expanding the environmental science offerings to students.

The Hanford Environmental Science and Engineering Consortium forms a critical segment of the pipeline, drawing students from predominantly minority institutions into research participation and training at the Laboratory. The partnership is dedicated to preparing increased numbers of environmental science and engineering professionals to address the myriad complex environmental problems facing the nation. HESEC comprises 1) the major Hanford contractors including Pacific Northwest National Laboratory, Westinghouse Hanford, ICF Kaiser Hanford, and the Hanford Environmental Health Foundation, 2) five Historically Black Colleges and Universities including Alabama A\&M University, Clark Atlanta University, Florida A\&M University, Southern University, and Xavier University, and 3) Heritage College.

A goal of the Consortium is to improve each member institution's capability to produce trained and employable graduates in environmental sciences and engineering building on the combined resources of the Consortium members and the U.S. Department of Energy's Hanford Site. HESEC provides support for internships at Hanford contractor sites for students and faculty at the member institutions, as well as investments in curriculum reform and basic research projects. HESEC also provides outreach to the precollege communities surrounding the member academic institutions. The Consortium was established in 1993 and 1994, and continued growth will depend largely on available resources.

Other Institutions with Predominantly Underrepresented Populations In addition to partnerships with Historically Black Colleges and Universities (HBCUs) represented in HESEC, the Laboratory has Memoranda of Understanding with the following: Howard University, North Carolina A\&T University, Prairie View A\&M 
University, and Tuskeegee University. The Memorandums of Understanding allow the Laboratory and the institutions to develop joint programs in science and engineering that include collaborative research projects, scientist and faculty visits and exchanges, and student internships. In addition, the Laboratory has provided seed monies for developmental research, supports Laboratory staff to serve as lecturers and workshop presenters, and assists HBCUs in designing precollege programs to attract and retain students in science and engineering studies.

Plans for FY 1997 and beyond include maintaining support for education and training at HBCUs as well as developing stronger ties to regional institutions of higher education that have predominantly Hispanic or Native American students.

Programs for Women To recruit and retain women in science and technology, the Laboratory continues to develop programs that link the Laboratory's resources with women students. In 1995, the Laboratory again conducted "Take Our Daughters to Work Day," inviting young women in grades six through eight to spend a day learning about their options in the world of work, particularly in science and technology. Again in 1995 as in past years, Laboratory staff met with groups of young women as part of Expanding Your Horizons conferences conducted in the state of Washington. These conferences attract hundreds of young women and provide an opportunity for their interaction with women of achievement.

At the college level, the Laboratory provides financial and in-kind support for students participating in the Women in Engineering Program at the University of Washington.

Programs for Disabled Continuing in FY 1996 is an effort to share science and scientists with students who are disabled or otherwise place bound. In partnership with a National Science Foundation-funded program at the University of Washington, Laboratory scientists tutor students and communicate electronically with disabled students across the state who participate in the program. Beginning in 1996, University and Science Education Programs and Human Resources will pilot the Research Internships for Students with Disabilities (RAISE) Program. RAISE is a collaborative effort between the Laboratory and the Richland School District's Special Programs Department. The goals of the program are to 1) facilitate school-to-work transitions for high school students with disabilities as they move into adult employment, adding marketable skills, work experience, and self-esteem to their resume; 2) advance Pacific Northwest National Laboratory's diversity goals by developing Laboratory staff appreciation for the contributions of individuals with differing abilities; 3 ) broaden the Laboratory's educational contributions to the community by providing opportunities for a group of capable people who have traditionally faced difficulty obtaining employment; and 4) increase the participation of underrepresented populations in science, mathematics, engineering, and technology. In FY 1996, one student will participate in the RAISE Pilot Project.

\section{Promoting Systemic Reform}

The Department of Energy and its national laboratories are the customer and the patron of a large segment of the nation's scientific and technical work force and have a direct stake in the quality of science, mathematics, engineering, and technology education. Further, the missions of DOE can best be achieved within a community educated to understand and value scientific research and development.

Therefore, the Laboratory's education programs support the national priority for a systemic approach to educational reform, an approach not based on ef forts to change discrete parts of the system, but an approach intended to change the whole system so that all parts together support students achieving at the highest possible levels. The Laboratory's participation in systemic reform is aligned with the national education strategy, Goals 2000: Educate America Act, passed by Congress and signed by the President, which centers on systemic reform and calls for partnerships that link the schools with the community, business, and government stakeholders.

Laboratory staff serve on national, regional, state, and local advisory boards and working groups to establish and implement content and assessment standards for mathematics, science, and technology education. The Laboratory continues to work with public and private-sector organizations in the state of Washington to implement statewide systemic reform in mathematics, science, and technology education, including serving on the state's committee to develop science education standards. Overall efforts include teacher enhancement and preparation, curriculum and instruction, assessment of learning, accountability, and resource development.

Curricula Reform The Laboratory continues to promote curricula reform supported by DOE by conducting the National Teacher Institute in Materials Science and Technology. The intensive 20-day summer institute trains teams of teachers and administrators from across the country to implement in their school districts the materials science and technology curriculum developed over the past 9 years by Laboratory scientists in collaboration with teachers. Since 1986, the development of this applied academics curriculum has been supported variously by DOE, the U.S. Department of Education, the Northwest Regional Educational Laboratory, and the state education offices of Washington and Oregon. More than 21 school sites in Washington and schools in 20 states and U.S. territories are implementing the curriculum. This hands-on, minds-on approach to learning aligns with education research and best practices and parallels the way scientists and engineers uncover knowledge and solve problems.

Teacher Enhancement Because teachers are key to reaching students at all levels, the Laboratory continues to provide high-intensity training and research experiences or elementary, middle, and high school teachers using 


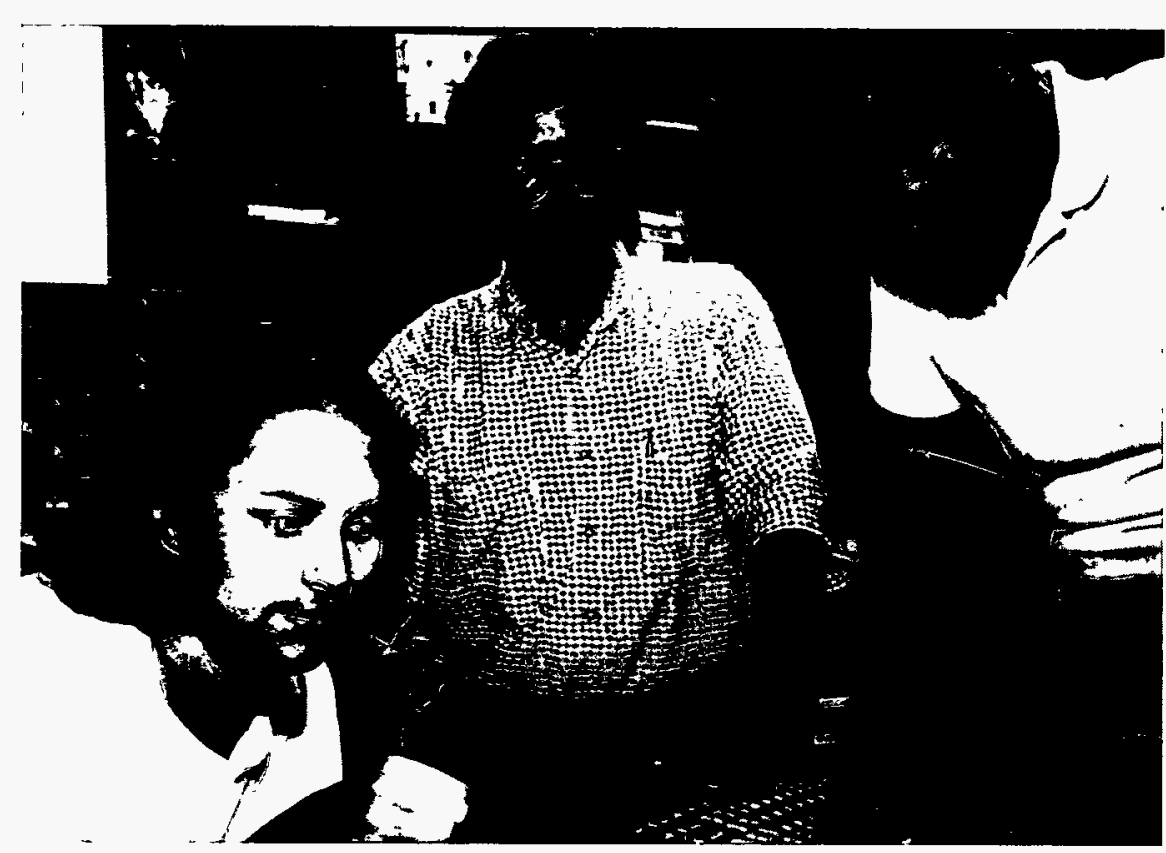

These TRAC participants teamed to develop a remote controlled robot with senses of vision, sound, and touch. They also developed programs for the computer interface.

Laboratory scientists, engineers, and equipment. At the elementary school level, the Laboratory received a second 4 years of funding from the National Science Foundation and DOE to implement the Science Alive program, a component of a DOE multilaboratory elementary teacher enhancement program. The second 4 years focuses on developing teacher-leaders who can implement change in their schools and districts. Teams of teachers from the Yakima Valley and Tri-Cities participate in summer institutes at the Laboratory and academic year workshops dedicated to producing long-term, sustained systemic change. Training includes 20 days in the summer and an additional 10 days during the academic year.

Based on Science Alive, the Laboratory has assumed responsibility for developing models for providing training and research opportunities for teachers throughout Washington State. Under a second National Science Foundation award to the University of Washington and support from Washington State Eisenhower funds, scientist-teacher teams from the Laboratory continue to assist in training scientist-teacher teams from three participating universities and the Pacific Science Center. Based in part on their training at the Laboratory, these teams in turn are providing training for elementary teachers throughout the state of Washington. The scope of this initiative is intended to affect not only the elementary teachers but the professional development of those college faculty who teach our future teachers and scientists.

Teacher Research Participation DOE's Teacher Research Associates (TRAC), a national DOE program for middle and high school teachers, has provided 8-week summer research experiences at the Laboratory for more than 280 teachers since 1986. This program has become a model for other federal agencies, universities, and industrial laboratories. Laboratory science education staff have assisted in the dissemination of the model program. The program strengthens the content knowledge and instructional strategies of teachers, providing an avenue for rapid transfer of frontier science to the classroom.

In summer 1995, the Laboratory continued a component of the TRAC program begun in 1994 to provide research experiences for teachers who are part of a National Science Foundation-funded master's degree project through Washington State University to enhance the abilities of chemistry teachers who are teaching out of their field of academic preparation. Also as part of TRAC, the Laboratory continues to host a teacher from the University of Washington's Math/Science Fellows (formerly Ford Fellows) program. The Laboratory also continued a teacher program begun in 1994 and funded under DOE's nuclear nonproliferation program to provide research experiences for teachers of science paired with teachers of the humanities. The outcome will be model instructional strategies based on the science, technology, and humanities content inherent in the teachers research experience.

Middle Level Systemic Reform The OPTIONS program, funded at the Laboratory since 1990 , aligns with statewide systemic reform in Washington State. Participating are middle schools having high percentages of underrepresented ethnic minorities. Each participating middle school has been challenged to do whatever it takes in the school and district to ensure that all students achieve at the highest possible levels in mathematics, science, and technology education. Each district has developed a plan for systemic reform and funds have been awarded to carry out the plans in collaboration with Laboratory research and education staff.

Over the next 5 years, we expect to extend the opportunity to additional schools and strengthen the link between OPTIONS and the state education reform. Outcomes of the evaluation and assessment are intended to reveal best practices that can be applied to advance systemic reform throughout the state and nation.

Student Incentives and Opportunities The Laboratory continues to conduct programs at all grade levels to provide students with incentives to study mathematics, science, and technology, and opportunities to participate in hands-on laboratory activities and interact with Laboratory scientists. Major precollege and university level programs for students are conducted 
at the Laboratory in Richland. The Laboratory also continues to participate in DOE's regional and national Science Bowl, providing judges, training for the regional judges, and questions produced by scientists. Most student programs are described under other headings within this section of the Institutional Plan.

\section{Educational Technology Initiatives}

Sharing Science with Schools, currently a DOE-funded classroom visitation program, will be expanded to include an electronic mentoring network between students, teachers, and Laboratory scientists. This component will provide a cost savings in travel and time as it augments the existing classroom visitation program.

The Computational Chemistry Pilot Project is a project that gives high school students the opportunity to work with computational chemistry databases as part of their advanced science studies at Kennewick High School. The project will link the high school students with the Environmental Molecular Sciences Laboratory. Students will perform molecular modeling calculations and submit them for inclusion in the official EMSL database.

Beginning in 1994, the Laboratory entered a partnership with the Kennewick School District which provided Internet access to Kennewick elementary and secondary teachers on a pilot basis. In 1995, Kennewick Schools and the Laboratory submitted a successful proposal to the Washington State Office of the Superintendent of Public Instruction (OSPI) to improve learning through the use of electronic technologies. The partnership, one of five selected from Washington State, will initially be directed toward providing Kennewick students and teachers remote access to and control of the Battelle telescope on Rattlesnake Mountain. Subsequent efforts will address other domains of science and technology at the Laboratory, including the Environmental Molecular Sciences Laboratory. The overall effort is supported by the National Science Foundation and coordinated by OSPI. Other partners in this initiative are Columbia Basin College, Heritage College, and the Alliance for the Advancement of Science Through Astronomy.

\section{Building Strong Laboratory- University Partnerships}

The Laboratory has a substantial and growing commitment to education and research partnerships with colleges and universities that spans nearly three decades. Through this broadly based initiative, the unique resources, staff, and facilities of this multiprogram national laboratory are shared with colleges and universities throughout the northwest region and across the nation.

Integral to building strong Laboratoryuniversity partnerships are the more than 1,100 students and faculty who participate each year in educational appointment programs at the Laboratory. Faculty research and education expertise is enhanced through such appointments which serve also to strengthen the academic institutions. Moreover, diverse research partnerships with academia enable the Laboratory to collaborate with a number of recognized experts in fields consistent with the business areas of the Laboratory and DOE.

\section{Agreements and Memoranda of Understanding}

Collaborative Agreements and Memoranda of Understanding provide a firm foundation for the Laboratory's partnerships with universities and are primarily intended to enhance collaboration between the Laboratory and universities by providing simplified and expanded administrative mechanisms. Partnerships strengthen the quality of research at participating universities through collaborative research, joint use of facilities and equipment, sharing of scientific equipment, appointments of university faculty as Pacific Northwest National Laboratory Affiliate Staff Scientists (PASS) appointees, and appointments of Laboratory staff as Adjunct and Affiliate Professors as well as guest lecturers at the universities.

The Laboratory has formal Collaborative Agreements with the following regional universities:

- University of Idaho

- University of Montana
- Montana State University

- University of Oregon

- Oregon Graduate Institute of Science and Technology

- Oregon Health Sciences University

- Oregon State University

- Portland State University

- University of Washington

- Washington State University.

Memoranda of Understanding are currently in place with the following universities throughout the country:

- California State Polytechnic University at Pomona

- Colorado School of Mines

- East Tennessee State University

- Eastern Washington University

- Heritage College

- Indiana University

- Mesa State College

- Pacific Lutheran University

- Reed College

- Rutgers University

- Saint Mary's College

- Sheldon Jackson College

- SUNY Buffalo

- Texas A\&M University

- University of Alberta

- University of British Columbia

- University and Community College System of Nevada

- University of Florida

- University of Kentucky

- University of Texas at El Paso

- University of Pennsylvania

- Utah State University

- West Virginia University

- William Marsh Rice University

- Yale University. 


\section{Science, Mathematics, Engineering, and Technology Education}

Pre-University Programs

Fiscal Year 1995

Student Programs

Student Research Apprenticeship Program

Inquiry Into Science

Marine Ecology Institute

CBC OPTIONS Scholars

Expanding Your Horizons/Career Conference

Sharing Science with Schools

Pacific Northwest National Laboratory Disabilities Opportunities

HESEC Summer Institutes (run by HBCUs)

Total

Women

Minority

Teacher Programs

Teacher Research Associates

Science Alive II (National Teacher Enhancement Project)

Summer Research Internship Program for Teachers

Materials Science and Technology Institute

Special Programs

OPTIONS Systemic Initiative

Washington Systemic Initiative in Science

Education - Science Teacher Enhancement Project

SUBTOTAL PRE-UNIVERSITY PROGRAMS

$\begin{array}{rrr}31 & 16 & 31 \\ 18 & 9 & 2 \\ 10 & 0 & 10 \\ 12 & 7 & 5 \\ 232 & 112 & 150 \\ 9,266 & 4,633 & 1,000 \\ 5 & 3 & 0 \\ 78 & 40 & 78 \\ & & \\ 37 & 15 & 5 \\ 29 & 23 & 3 \\ 5 & 1 & 0 \\ 30 & 10 & 6 \\ & & \\ 3,303 & 1,618 & 2,202 \\ 27 & 17 & 1 \\ & & 3,493\end{array}$

University Programs

Undergraduate Programs

Science and Engineering Research Semester (SERS)

Laboratory Cooperative Program

Alliance for Minority Participation (AMP)

GEM (minority)

HBCU Programs (minority)

HAMMER Scholars Program (minority)

HESEC (appointees, minority)

HESEC (scholars/scholarships, minority)

National Merit Scholars

Sheldon/Jackson (minority)

WSU-TC OPTIONS

Graduate Programs

Laboratory Cooperative Program

DOE Graduate Fellows

National Physical Science Consortium (minority)

Pacific Northwest National Laboratory Energy Research Fellowship

Postdoctoral Programs

Laboratory Cooperative Program

DOE Postdoctoral Program

Faculty Programs

Laboratory Cooperative Program

Pacific Northwest National Laboratory Affiliate Scientists

HESEC (minority)

SUBTOTAL UNIVERSITY PROGRAMS

GRAND TOTAL

68

254

2

2

3

15

18

94

2

2

1,127

14,210

31
94
1
1
1
9
9
31
1
2
2

51

1

1

5

28

0

16

13

8

305

6,809 
The partnerships are defined and executed jointly by the Laboratory and the college and university partners. Under the partnerships, 5-year Master Agreement subcontracts have been developed with 29 universities throughout the United States to facilitate collaborative research, and pre-qualify the universities in one or more of 15 technical work areas. Since Master Agreements were first instituted 7 years ago, joint research projects between the Laboratory and universities have increased significantly. During FY 1995, the Laboratory undertook more than $\$ 9.4$ million in subcontracts with universities, and the rate of growth is expected to increase steadily during FY 1997 and beyond.

Areas of joint activity under the Master Agreements include meteorological monitoring modeling and analysis, global change environmental monitoring and modeling, waste site characterization, waste treatment, computational sciences, sensor development, molecular and structural biology, chemical and materials science, toxicology, health physics, risk assessment, energy systems, infrastructure modernization and productivity enhancement, and technology planning and analysis.

Within the Master Agreement framework the Laboratory supports the establishment of new professorships at regional universities. In past years, the Laboratory assisted in establishing new faculty programs in surface science, chemical engineering, and environmental engineering at Washington State University and the University of Washington. During FY 1997 and beyond, the Laboratory expects to establish faculty programs and support curriculum development in advanced areas of environmental science and molecular science at regional universities.

The Laboratory has recently initiated the DOE-funded Environmental Science and Engineering Education Coordination and Assistance Project (ESEECAP). The purpose of this program is to assist Washington State University Tri-Cities (WSU-TC) to provide relevant and timely educational programs in environmental science and engineering to Hanford staff and the local community. A bachelor's degree program in Environmental Science was recently initiated, and a master's degree program has been under way for several years. These programs provide an applied approach to education designed to equip students with the technical background and hands-on knowledge to address environmental problems that are related to Hanford as well as to regional, national, and worldwide concerns.

Postdoctoral Fellowships Postdoctoral fellows play a key role in Laboratory research activities, particularly in molecular sciences. The Laboratory's activities at the postdoctoral level have grown significantly during 1995 , and represent the largest growth in university programs at the Laboratory. The DOE Lab Coop program provided 1- and 2-year fellowships to more than 175 outstanding postdoctoral researchers.

Also participating in research under the guidance of Laboratory scientists and engineers are those outstanding researchers who have received national DOE postdoctoral fellowships including Alexander Hollaender, Human Genome, Global Change, and the Distinguished Postdoctoral Fellowship. These highly competitive DOE fellowships provide the Laboratory the opportunity to work with some of the best new researchers in the country, while also expanding the Laboratory's contacts with universities connected to the past work of the fellowship recipients.

Faculty Preparation and Enhancement The Laboratory, jointly with AWU NW, actively promotes opportunities to advance the knowledge and skills of university faculty in science and engineering. The intent of these programs is to bring fresh ideas into the Laboratory, and to stimulate the development of updated curricula that draws on interdisciplinary approaches at the Laboratory.

The PASS program has undergone significant changes during FY 1995 and is expected to become a premier program during FY 1996 and beyond. PASS allows Laboratory researchers to nominate university faculty collaborators for PASS membership, thus helping to formalize and enhance collaboration. PASS members are allowed access to Laboratory facilities, as well as travel support provided by the host researcher. It is expected that the PASS appointments will continue to open the Laboratory's doors to university researchers and stimulate new ideas and research opportunities.

A counterpart to the PASS program, Laboratory staff members hold faculty joint appointments at university campuses. More than 240 affiliate professorships and lectureships, approximately one-half of which are held at university campuses around the country, are held by Laboratory staff. The remaining one-half are held at regional universities including WSU-TC located in Richland.

\section{Evaluation and Capacity Building}

Building on the past 3 years of participation in DOE's project to assist the laboratories in evaluating the quality and assessing the impact of their education programs nationwide, the Laboratory expects to formalize a multifaceted approach to ensuring that goals and objectives are met for the full array of the Laboratory's education programs. This opportunity for capacity building has added value to the education programs at the Laboratory, strengthened our role through the state and region, and improved the abilities of education staff to conduct effective programs. In addition, the outcomes and results of program evaluation and impact assessment provide tools for the nation to identify effective programs that connect students and faculty with the resources of DOE laboratories or similar science and technology organizations.

\section{Environmental Quality}

Management and staff at Pacific Northwest National Laboratory have a major role in DOE's Environmental Quality programs. The dominant part of our research and development activities are devoted to resolving environmental issues faced by DOE and other public and private organizations. Our work is largely devoted to supporting the 
Department in meeting the demanding requirements for waste management and environmental remediation across the DOE complex, as well as meeting the nation's environmental needs.

Our science programs are helping to provide the knowledge required to effectively manage waste and remediate environmental contamination at Hanford and across the DOE complex. Our technology development programs are providing technologies that reduce the cost or improve the timeliness and effectiveness of DOE's cleanup program. We are also working to supply the knowledge, methods, and systems engineering capabilities required to support DOE in the risk-based decision making required to set priorities and select technical approaches. Finally, we are committed to providing the scientific understanding and the technical methods that enable DOE to ensure the protection of its workers and the public in DOE operations and that support the establishment of risk-based environment, safety, and health standards.

In meeting these responsibilities, we recognize the necessity for partnerships with other laboratories, industry, and academia. No single institution brings the full suite of capabilities necessary to resolve the most complex problems facing DOE. Our major environmental programs involve the broad collaborations necessary to deliver comprehensive solutions to DOE's most complex and most pressing needs.

The Environmental Quality section provides details on Laboratory support to DOE-EM. Work on integrated and risk-based environmental planning for Hanford as well as most of the DOEEM Program Offices is described first. The next section describes Pacific Northwest National Laboratory's support to the management of the National Technology Development Program as well as its technology development and deployment roles within that program. The section following discusses the Laboratory's role in supporting the application of environmental technologies to the environmental restoration and waste management programs within DOEEM. The application of these technologies is primarily targeted to the Hanford Site, but also includes support to DOE-HQ and other DOE sites. The Environmental Quality section concludes with a discussion of Pacific Northwest National Laboratory's EM facility activities and its specific activities supporting Hanford Site operations.

\section{Office of Environmental Management}

The majority of the Laboratory's support to DOE in the environmental area is to the Office of Environmental Management. This support includes a broad range of technological work such as basic studies on waste interactions, systems engineering, development of risk management approaches, regulatory and policy analyses, environmental monitoring, technology development and deployment, and actual cleanup operations. While the Laboratory's primary support is to DOE-EM programs at Hanford, we also support environmental activities at other DOE sites, as well as a number of components of the national DOE-EM program.

We recognize the necessity and importance of linking our DOE-ER activities to programs supporting DOE-EM. We expect that the fundamental research the Laboratory is conducting will increase our understanding of complex environmental systems, thereby leading to new technologies that will fill technological gaps in the current cleanup program or provide new solutions which can reduce costs and increase effectiveness. The Laboratory is working to focus its EMSL research program on DOE's environmental restoration and waste management needs. This effort will strengthen the contextual framework for this state-of-the-art facility and is expected to result in breakthrough solutions for critical environmental problems.

The estimated funding for DOE-EM is shown in the Resource Projections section. The funding levels indicated also include work done by Pacific Northwest National Laboratory for the Westinghouse Hanford Company and the funding projections for the $\mathrm{DOE}$ EM operating portion of the EMSL.

\section{Integrated Risk-Based Environmental Planning}

The complex environmental challenges facing the Department of Energy cannot be dealt with one at a time. The problems are interrelated, and the solutions carry different probabilities of success and different potentials for further damage. To evaluate these factors, the Laboratory has developed systems engineering methods to integrate the planning activities supporting the different components of the DOE-EM program and risk management approaches to systematically address the different aspects of risk and environmental decision making. This section addresses the integrated planning and support to Hanford as well as national DOE-EM Program Offices. It also discusses risk management approaches and the associated methods and tools for assessment of management and information.

\section{Hanford Integrated Planning and} Support The Hanford Site represents one of the most complex environmental cleanup tasks faced by this country. Transition from Hanford's former production mission to its present cleanuporiented mission has not been easy. Among the challenges that must be overcome are 1) unclear endpoints for cleanup; 2) a complex regulatory environment with many federal, state, and local agencies having authority over cleanup; and 3) highly complex tasks that are beyond the capability of existing technology or the nation's willingness to pay.

The Laboratory is supporting DOE-RL in managing cleanup as an integrated project. Formal systems engineering methods are being used to define what work must be accomplished by each element at Hanford. Systems and risk analysis methods are being applied to evaluate the impacts of both alternative endpoints for cleanup and alternative approaches for reaching those endpoints. The Laboratory has recently provided the first assessment of public, worker, and environmental risks 
from a total site perspective. This assessment provides a basis for understanding the contribution to risk from different elements of the cleanup program (e.g., high-level tank waste, buried solid waste, existing soil and groundwater contamination), and provides the basis for setting endpoint cleanup targets that are driven by credible scientific information.

Insights gained from this integrated view of Hanford cleanup will define requirements and opportunities for improvements in basic science and cleanup technologies. These improvements will enable ultimate cleanup endpoints to be reached in the most timely and cost-effective manner. Continuing the Laboratory's strong role in Hanford integrated planning over the long term will help ensure that technology and science investments are focused on real needs and that cleanup dollars are applied to achieve real progress.

Technology Planning and Evaluation Support Pacific Northwest National Laboratory is developing systematic approaches to understand the magnitude of DOE's environmental problems, the interrelationships and interfaces important to cleanup and waste management activities, mechanisms for prioritizing activities, methodologies for analyzing problems and solutions with multiple stakeholder participation, and mechanisms for evaluating progress against DOE-EM's goals.

The objective is to develop needsdriven, cost-effective programs by evaluating and reducing short- and long-term human health risks, and protecting/improving the quality of natural ecosystems found on DOE sites. To improve DOE's allocation of its environmental management resources for maximum risk reduction, the Laboratory is assisting DOE in developing risk-based assessments for site cleanup scenarios and options, and factoring risk impacts into DOE's decision making and environmental management policies and programs.

The Laboratory provides substantial support to DOE-EM headquarters activities, both in planning and evaluation activities within specific offices

\section{FLC Award for Excellence in Technology Transfer}

Laboratory staff members took an expensive electronic personnel dosimeter and re-engineered it into an affordable, highly sophisticated portable radiation analyzer. The fractional channel multichannel analyzer is a hand-held instrument that allows field technicians to instantly monitor radioactivity levels onsite, eliminating the costly process of gathering, analyzing, and disposing of samples.

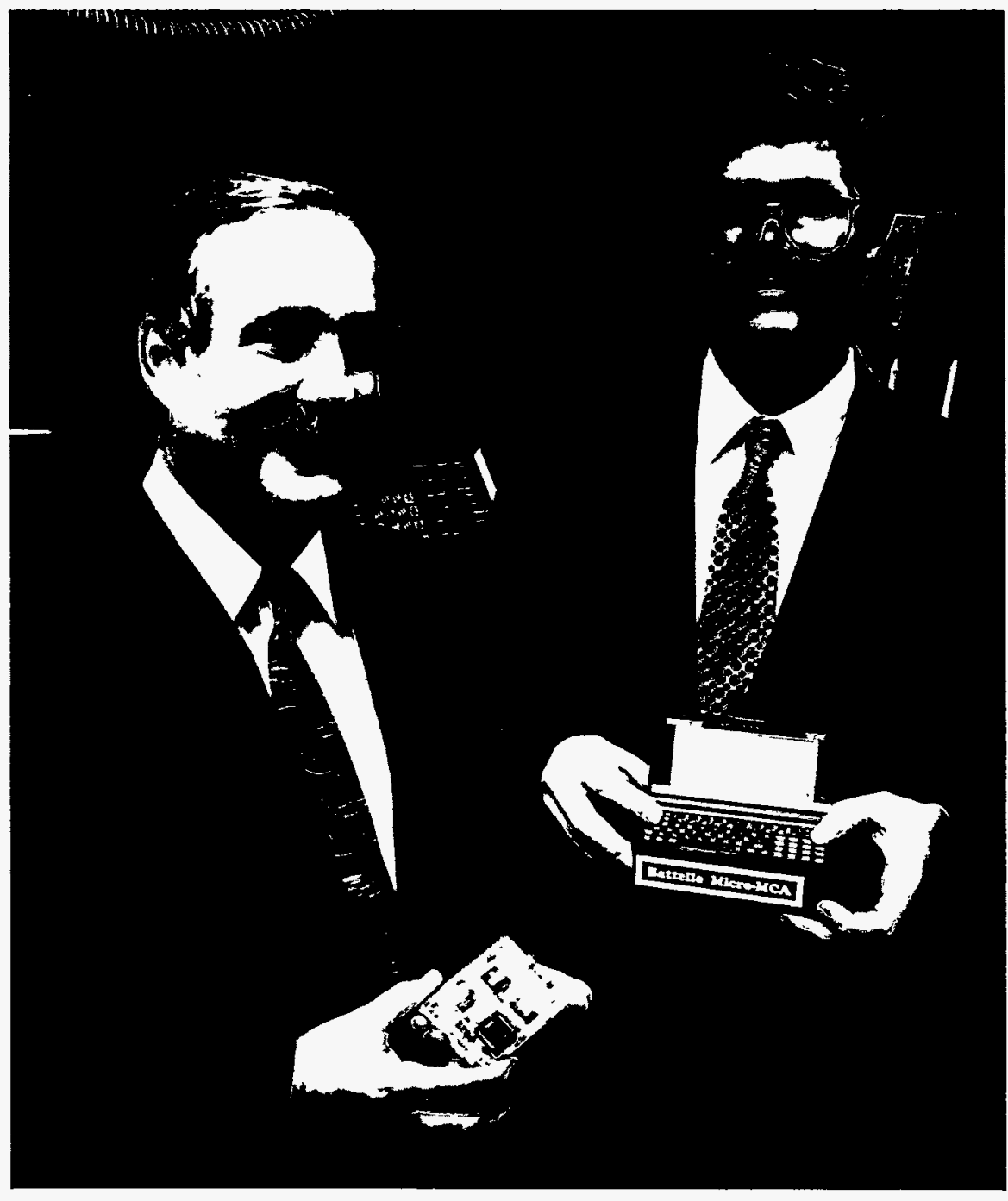

and in crosscutting activities intended to set the future direction of the office. This type of analysis and information development support has gained the Laboratory a reputation for understanding the detail of DOE-EM operations across the complex and using that knowledge and our capabilities to provide analytic results to help the highest levels of DOE-EM make key policy decisions. Examples of this type of crosscutting activity include the Laboratory's support to the Programmatic Environmental Impact Statement, the Baseline Environmental Management Report, and the report to Congress addressing the risk, cost, and stakeholder issues associated with compliance agreements to which DOE has agreed. 
The Laboratory has also been supporting specific offices within DOE-EM to develop forward-looking plans. For example, we supported the Office of Oversight and Self-Assessment (EM-20) through the development of comprehensive EM-20 strategic plans, individual office implementation plans, and office-specific program/activity plans. These plans ensure that project activities are implemented in a coordinated fashion to meet DOE-EM objectives. Other project activities included regulatory analysis, technical document review, work on the national compliance plan, stakeholder involvement (particularly with respect to the revision of DOE Order 5820.2A), strategic change management, emergency management assessments and reviews, analysis and implementation of DOE American Indian policy relative to transportation and emergency management issues, and participation in the Analytical Services Program.

Support to the Analytical Services Program involves two major activities: methods compendium development, and data quality objectives training and support. Pacific Northwest National Laboratory prepares and distributes DOE Methods for Evaluating Environmental and Waste Management Samples. In addition, the Laboratory participates in national meetings and conferences, prepares information reports, maintains databases supporting sampling and analytical needs at Hanford, implements processes to review collected information, applies strategies to reduce costs, identifies priority needs for Laboratory support, and identifies DOE analytical method and capacity shortfalls. The Laboratory also supports DOE's efforts to ensure that data collection activities are decision-based and that uncertainties are managed appropriately through implementation of data quality objectives planning methods. This scope encompasses data quality objectives training, statistical methods research, sampling and analysis tools development, DOE statistical clearinghouse, and data quality assessment.

Pacific Northwest National Laboratory also provides technical planning support to DOE's Office of Waste Management (EM-30). Laboratory staff provide input into the development of a systematic approach to manage low-level radioactive mixed wastes across the DOE complex. The Laboratory is also assisting DOEHeadquarters in the evaluation of the operations offices' programs to ensure the compliant shipment of DOE's hazardous waste to offsite commercial disposal facilities.

The Laboratory provides planning and evaluation support to DOE's Office of Environmental Restoration (EM-40) in the following areas:

- identifying and analyzing current and emerging regulatory and institutional issues of concern to DOE's environmental restoration program (e.g., institutional controls, land disposal restrictions)

- identifying programmatic approaches and options for expediting site cleanup and providing support to field organizations in implementing streamlined approaches. Activities include 1) collaboration with DOE operations offices and U.S. Environmental Protection Agency (EPA) regulators, 2) development of strategies for the integration of phased remedial investigation/feasibility studies, 3) analysis of options for expediting site cleanup through the use of Resource Conservation and Recovery Act (RCRA) corrective action authority, and 4) assessing DOE Orders that could be revised to expedite remediation activities.

- supporting the DOE-EM Programmatic Environmental Impact Statement including collection of source-term descriptions and environmental settings data for restoration sites, development of a complex-wide inventory of DOE's environmental restoration sites, engineering analysis, regulatory analysis of compliance agreements, and development of modifications to multimedia environmental pollutant assessment models.

Pacific Northwest National Laboratory supports the Office of Facility Transition and Management (EM-60) as a part of DOE-EM's decontamination and decommissioning objective. The Laboratory provides support and guidance to EM- 60 by analyzing and reviewing policies and procedures established for its operation, methods by which it links to field organizations, and coordination with other $\mathrm{DOE}$ and federal organizations. Key to this support is the development of guidance and acceptance procedures that govern acceptance of facilities into DOE-EM from their former owners, ensuring safe and cost-effective transition of these facilities.

\section{Integrated Risk Management}

Pacific Northwest National Laboratory has been at the forefront of promoting integrated risk management as a key method for establishing priorities and managing DOE's environmental activities. The Laboratory was involved in the initial effort to establish the Office of Integrated Risk Management (EM-6) and continues to support such efforts. The Laboratory also continues to work to establish programs at Hanford that will assess risk from alternative strategies for remediation activities in terms of risk to workers, the public, and ecosystems. Three major elements or products of these programs include

- quantitative assessment of public health and worker safety and health risks for materials currently present on the DOE complex

- quantitative assessment of the public health, worker, and ecosystem risk that will result from remediation activities under alternative strategies for cleanup

- quantitative assessment of public health, worker, and ecosystem risk associated with remediation activities.

Beyond establishing this core information, a key question is how to use risk assessment and risk management information in planning cleanup actions. The benefit of a risk management approach to planning is to demonstrate what each DOE-EM action, policy, regulatory driver, or new technology does in terms of cleaning up sites and 
impacting human health or the environment and then to apply that information to key DOE decisions. The Laboratory has been actively involved in this process, developing such approaches as the Priority Planning Grid for application to the budget allocation process at Hanford. We are also developing tools to support these initiatives at headquarters, including methods for assessing risks and costs of strategic options for cleanup at the complex level of analysis.

\section{Measurement Methods/Tools \\ Development and Information \\ Management Pacific Northwest}

National Laboratory provides support to DOE-EM in environmental modeling, data analysis, risk management, prioritization, information management, systems analysis, and education.

The Tank Waste Information Network System (TWINS) links site-specific tank information from Rocky Flats, Oak Ridge, Savannah River, Idaho, and Hanford into a common system that is available to multiple users. Tank data exists in many forms-hard-copy records, isolated personal computers, and fully networked onsite databases. Such data cannot be retrieved easily or shared with other users efficiently. By linking different databases, TWINS allows users to access, manipulate, and retrieve tank data from any of the high-level waste tanks at DOE sites.

The Hanford Environmental Information System (HEIS) has been designed and implemented to store, manage, and aid in the analysis of Hanford's environmental sampling data; activities which are crucial to environmental cleanup and restoration. The HEIS is a repository for storing data and includes tools for efficient data processing, mapping, and spatial data analysis; maps of the Hanford Site; and software for entering, viewing, reporting, and depicting data. HEIS is operated by the Laboratory as a resource for the entire site.

The Laboratory is developing a computer-based advisory tool that identifies, screens, links, and evaluates established technologies and unit processes for the remediation of operable units and waste management units. The tool, RAAS (Remedial Action Assessment System), will be used primarily in support of feasibility studies under the Comprehensive Environmental Response, Compensation, and Liability Act (CERCLA) (or corrective measures studies under RCRA), and is intended to help implement established and newly developed technologies that emerge from demonstration, testing, and evaluation.

\section{Development of Environmental Technologies}

One of the Laboratory's primary objectives is the development and ultimate deployment of new technologies that can significantly benefit DOE environmental programs. The Laboratory has been able to make significant contributions toward this objective through its involvement in DOE-EM's National Technology Development Program. The Laboratory is responsible for both leadership roles in several of the new technology development focus areas, and for the conduct of a number of technical tasks to provide new technologies. These tasks include the responsibility to ensure that these technologies are deployed in DOE-EM programs and broader markets. This section describes the Laboratory's support to the National Program, its recent and planned deployment successes, and its approach to technology implementation.

\section{Support to the National Technology} Development Program Pacific Northwest National Laboratory is participating in DOE-EM's National Technology Development Program by providing technical management support and performing technology development tasks for several focus areas. The Laboratory has been assigned the lead for the Implementation Team supporting the Tank Focus Area, and Laboratory staff are performing several key roles in management of the Plume and Landfill Focus Areas. The Laboratory and other Hanford staff are working with Idaho National Engineering Laboratory to identify how Hanford technical and human resources can support the Mixed Waste Focus Area, which Idaho National Engineering Laboratory is leading. Pacific Northwest National Laboratory remains responsible for coordinating the Efficient Separations and Processing Crosscutting Area. The Laboratory also has retained responsibility for program coordination and several key tasks in the Robotics Tank Waste Retrieval element of the Robotics Technology Development Program, which also will continue as a crosscutting program. Details on the Laboratory's roles are provided below.

Tanks Focus Area The Laboratory leads the Implementation Team supporting the Tank Focus Area, coordinating its functions and providing technical management, decision support, and reporting functions associated with the technical program. The Implementation Team, which began operation October 1994, is composed of the Idaho National Engineering Laboratory, Los Alamos National Laboratory, Oak Ridge National Laboratory, Sandia National Laboratories, Savannah River Site, and Westinghouse Hanford Company. DOE-RL serves as the lead operations office and administrator of this team. The overall program objective is to deliver a tanks technology program that is applicable, integrated, acceptable, and accountable. Pacific Northwest National Laboratory's Technology Development Program works with the Tank Focus Area team and the Hanford Site Technology Coordination Group (representing users and stakeholders) to ensure that the resulting technology development program meets Hanford's user needs, has a sound technical basis, and meets stakeholder expectations.

DOE faces a major tank remediation problem-approximately 250 tanks storing approximately 380,000 cubic meters of high-level waste across the DOE system must be remediated and made safe. The tank waste exists in different forms including solids, liquids, sludges, and slurries. The constituents vary across the four major tank sites (Idaho, Hanford, Savannah River, and Oak Ridge) and within tank farms at each site. Some tanks contain organic chemicals that generate gas or 
high heat and may be potentially explosive. The tanks also differ in structure, composition, and capacity. At present, 79 of these tanks are known or assumed to have leaked waste to the environment. The technical risks of remediation are complicated by programmatic, institutional, and regulatory issues that also vary across sites. Prior to FY 1995, responsibility for developing technologies supporting tank remediation was spread across multiple organizations and sites within the DOE system.

While remediation baselines and systems engineering concepts differ across sites, the high-level waste tank remediation problem can be broken down into six common remediation functions: safety, characterization, retrieval, pretreatment, immobilization, and stabilization and closure. The Tank Focus Area technical team is organized around these functions, with a Technical Integration Manager from each of the member organizations assigned to each function. Pacific Northwest National Laboratory coordinates these functions and provides management, decision support, and reporting functions associated with the technical program.

To ensure that the resulting technology development program meets user needs, a User Steering Group composed of senior managers of the tank remediation programs at each of the major sites has been established and reviews Tank Focus Area products at regular intervals. To ensure that the program has a sound technical basis, a Tank Focus Area Review Group composed of technical experts from industry, universities, and national laboratories has been established and will review the program from a technical perspective. To ensure that the program meets stakeholder expectations, each site has established a Site Technology Coordination Group to help establish a listing of prioritized needs and to review technical products.

To define the technical program, the Tank Focus Area Technical Team collects and validates each site's tank remediation needs, prioritizes the needs and develops a technical strategy for responding to them, and defines a responsive technology development program that can demonstrate clear return on investment.

These activities have produced a national comprehensive database on tank remediation technology needs and technical activities that may address those needs. During FY 1995, the Tank Focus Area Team delivered a Multi-Year Program Plan presenting its technical strategy and program, a call for proposals to support that plan, and a Fiscal Year Work Plan for FY 1996 activities. In FY 1996, these plans will be updated and the Tank Focus Area Team expects to broaden its scope to include cognizance of activities beyond DOE-EM that are relevant to tank remediation and to more closely coordinate and manage relevant EM activities.

Work in the Tank Focus Area currently supports, or could support, several projects within the Spent Nuclear Fuels program. This support includes demonstrations of decontamination and decommissioning technologies, information useful to systems study for the sludge-path-forward, characterization (potential applications for the Laser Ablation technology), vitrification and repository waste form development, and waste acceptance processes. The anticipated long-term benefits of Tank Focus Area support to the Spent Nuclear Fuel program will be in the processing required beyond the interim storage period. In addition, technology cross pollination is expected in the remote handling/robotic development areas. ALARA and process optimization due to implementing improved remote handling systems in the stabilization facility can also be expected. Laboratory staff supporting both of these programs will be working together to bring about these and other joint benefits. Laboratory activities supporting the Spent Nuclear Fuel program engineering and operations organizations are described in the Waste Management Technology Programs section.
Robotics Crosscutting Area The robotics tank waste retrieval effort will continue for several years, focusing technology development efforts on tank waste retrieval applications. Specific technical areas are improved autonomous controls and sensing, better interpretation of sensor data, and new end-of-arm tooling. Accomplishment of these goals will lead to improved productivity and greater safety for workers and the public. Pacific Northwest National Laboratory will continue to provide technical support to this effort, as well as overall coordination of the system-wide tasks supporting retrieval and the large, consolidated demonstrations of robotic retrieval systems in a National Robotics User Facility sited at Hanford.

Plumes and Landfill Focus Areas Over the next 5 years, Laboratory staff will manage and coordinate a number of activities and functions in support of the Implementation Team Lead Organizations at the Savannah River Site for the Plumes and Landfill Focus Areas. Two staff members are on the core management team with responsibilities for technical management and external integration. Additionally, several other Laboratory staff are assigned key roles such as product line manager, technical coordinator, and various support specialists. This effort is related to ongoing Laboratory coordination responsibilities for old EM-50 program elements (i.e., VOC-Arid Integrated Demonstration and the In Situ Remediation Integrated Program), which phased out and transitioned into the new focus areas during FY 1995.

\section{Efficient Separations and Processing} Crosscutting Area Laboratory staff are providing key technical and coordination support to the Efficient Separations and Processing Crosscutting Area, which supports each of the current problem-directed focus areas. The program's original emphasis on highlevel waste separations is still apparent, but is decreasing compared to other focus area needs. Over the next 5 years, more emphasis will be placed on separation processes for low-level 
waste and hazardous chemicals. Efficient Separations and Processing will develop separations technologies for a spectrum of radioactive and hazardous defense wastes with a goal of transferring the technologies to industry to facilitate U.S. competitiveness in the world market. In addition, advanced separations technology development is a major focus at the Laboratory and is a key component of the Advanced Processing Technology initiative.

\section{Hanford's Site Technology Coordination Group Implementation} Tom Grumbly's A New Approach to Environmental Research and Technology Development at the U.S. Department of Energy (the New Approach) calls for an integrated team structure that includes a Site Technology Coordination Group (STCG) at each DOE site to consolidate the site's technology needs, enhance communications, and provide technology transfer functions. The STCG structure implemented at Hanford consists of a Management Council and four subgroups: 1) Plumes and Landfills, 2) Tanks, 3) Mixed Waste, and 4) Decommissioning and Decontamination. The New Approach mandates directly linking technology development activities to specific site cleanup needs and involving regulators, stakeholders, and potential users in the technology development selection and implementation process. Three goals have been developed for the Hanford STCG

- establish the Hanford STCG as the lead authority and corporate voice for the site's technology needs assessment, as well as technology development, demonstration, and deployment

- coordinate with the new Focus Area Implementation Teams. Provide advocacy for the site's needs within DOE-EM's National Technology Development Program and ensure that those efforts are responsive to Hanford's needs.

- coordinate technology development and acquisition for the site. Accelerate technology acceptance by involving regulators and stakeholders

\section{R\&D 100 Award Winner}

Electrical Remediation at Contaminated Environments-This quick and inexpensive technique uses electrodes in situ to heat natural moisture in the soil. The steam which is produced removes contaminants from the soil. This technique does not require excavation or soil pretreatment. Soils that would otherwise require years to clean can be cleaned in weeks with this technology.

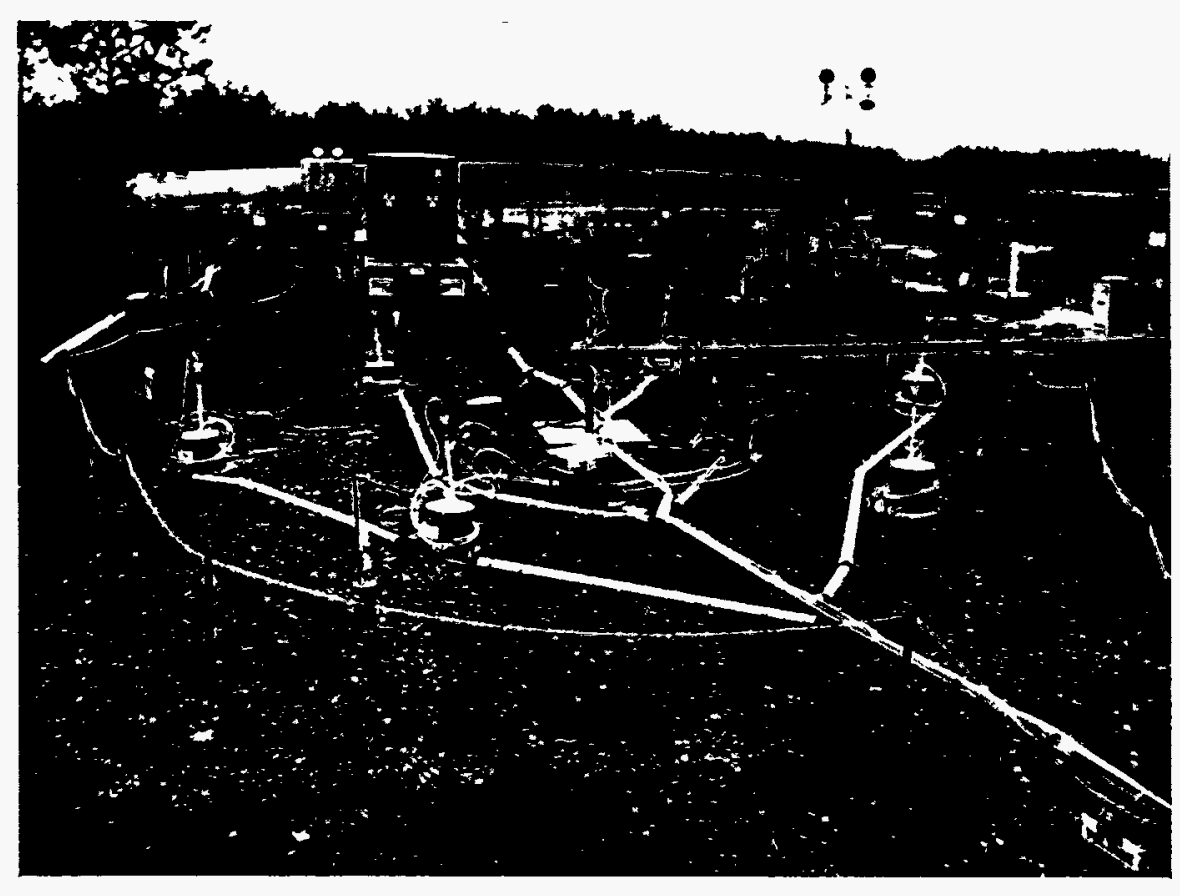

early in the development and selection process. Identify operational schedule drivers and technology insertion points.

Pacific Northwest National Laboratory, along with each of the other Hanford contractors (Westinghouse Hanford Company and Bechtel Hanford Company), has an ex officio member on the STCG Management Council. In addition, Laboratory staff and other contractors will participate in one or more of the focus area subgroups to provide specialized technical expertise. Since the Laboratory is also playing key roles in four of the DOE-EM Technology Implementation Teams, its STCG members can also provide the Management Council with technical input on how to best respond to Hanford's highest-priority technology needs and how to leverage the results of DOE-EM's planned development and implementation activities. The Laboratory's STCG support staff will also be able to provide Bechtel Hanford, which has no EM-50 office, information on the status of the EM-50 program, including STCGs at other sites, Focus Areas, and technologies available for demonstration/deployment.

Technology Development and Implementation Roles Pacific Northwest National Laboratory is performing technology development tasks for all of the EM-50 program areas. Specific technologies under development include new analytical techniques for rapidly analyzing the highly radioactive tank wastes, development of new separation agents for treating tank wastes, new characterization devices for mapping the distribution of contaminants in the soil, subsurface manipulation processes to form containment barriers for contaminants in the soil and groundwater, enhanced contaminant extraction combined with new surface destruction techniques, soil and groundwater treatment with biological 
methods, membrane technology to remove tritium from groundwater, plasma arc technology for treating excavated solid wastes, and models for assessing subsurface performance and risks. The Laboratory's preferred approach is to conduct these tasks jointly with users, concerned stakeholders, and industrial partners. Approximately one-third of the Laboratory's EM-50 dollars go to universities and industry. The goal of these partnerships is to accomplish implementation of the solutions as quickly as possible. The Laboratory has, or is now developing, through the EM-50 program and other programs, over 130 environmental technologies.

Near-Term Technology Development Targets A major objective of our technical tasks is to rapidly transfer technologies to DOE-EM's operating programs. The Laboratory has implemented or is transferring a number of technologies, and other technologies are being closely coordinated with the operating programs to ensure rapid transfer will occur. For example, EM-30 and EM-50 are jointly funding development of in situ and ex situ technologies for analyzing Hanford tank wastes. In each case, the researchers who developed the technology for EM-50 are working with the operating contractor to transfer the technology or to develop the joint partnership arrangements. A list of selected deployment successes is included in a section below, and several technologies under development now which are expected to be deployed over the next several years follow.

Waste Dislodging and Conveyance removes the solid "heel" that remains in tanks after soluble waste has been removed. Water-efficient scarifiers break up the solid waste, and a vacuum or water jet pump conveyance moves the chunks out of the tanks without significantly increasing the volume of waste water. Demonstrations are planned at four DOE waste tank facilities.

Graphite-Electrode Arc Furnace vitrifies solid, liquid, and off-gas wastes. Graphite electrodes create an arc used to heat up to 10,000 pounds per hour of feedstock to over $1700^{\circ} \mathrm{C}$. The resulting slag cools to a glass-like matrix that is extremely durable. A number of diagnostic tools developed for this task can be broadly applied by industry for emission monitoring and unique temperature measurements.

In Situ Redox Manipulation creates a permeable treatment barrier in the subsurface by the injection of reagents and/or microbial nutrients. The reagents or nutrients alter the reduction/oxidation (redox) potential of the aquifer to destroy, immobilize, or mobilize contaminants migrating through the manipulated zone. A field demonstration was planned for FY 1995. The Hanford Site is including this technology in the 5-year plan.

Chemically Reactive Subsurface Barriers are permeable barriers that act as selective filters to remove contaminants such as strontium-90 from flowing groundwater. These barriers are being developed as an effective, low-cost method to remediate contaminated subsurface environments.

\section{Laser Ablation/Mass Spectrometry} is a prescreening system that gives elemental analysis and molecular speciation information on core samples of tank waste. This technology greatly reduces sample analysis costs.

In Situ Bioremediation is a method of injecting nutrients into groundwater to stimulate naturally occurring microorganisms into acting upon and breaking down organic contaminants. The process is currently being demonstrated at Hanford.

As noted above, over the next 5 years the Pacific Northwest National Laboratory Technology Development Program expects to bring the six major systems listed above as well as a number of other smaller technologies into deployment. Other expected future accomplishments include having one or more monitoring/characterization technologies routinely supporting the characterization of Hanford's high-level waste tanks, several in situ subsurface barrier technologies demonstrated as suitable interim stabilization measures, and an integrated set of technologies identified for deployment through industry in the decontamination and decommissioning of nuclear fuel storage basins.

Recent Technology Deployment Successes Over the last year, Pacific Northwest National Laboratory saw a number of its technologies deployed in actual environmental operations or accepted by industrial organizations for implementation. Following is a listing of recent deployment successes.

Six-Phase Soil Heating simultaneously heats and ventilates soils to enhance removal of volatile and semivolatile organic compounds. This technology cleans soils to regulatory requirements outlined in RCRA and CERCLA. It was demonstrated full-scale at Savannah River and is being used to treat a contaminated site at the Rocky Flats Plant.

In-Situ Vitrification electrically heats and melts soil contaminated with radioactive, chemical, and organic waste. Organics are destroyed by the heat, other contaminants are immobilized in the solid block of obsidian-like glass that remains after cooling. This technology is being used commercially, and EM-50 support helped expand the operating envelope and resolve technical issues limiting technology deployment.

Ground Penetrating Holographic System generates high-resolution, threedimensional images of buried waste in real time. This system is currently being used to define several subsurface structures at Hanford before remediation work starts.

High Energy Corona can be applied to many liquid or gas wastes containing hazardous organic compounds. A highenergy electrical field creates reactions that destroy the contaminant molecules or reduce them to nontoxic materials. This technology was fieldtested at the Savannah River Site and a demonstration as a pollution prevention device was planned for 1995 with an industrial partner. 
Electromagnetic Induction Moisture

Method is designed to monitor moisture content and liquid levels in Hanford's

high-level waste storage tanks.

Deployment of a prototype was

planned for 1995.

Waste Acid Detoxification and Reclamation removes metals and recovers reusable acid from industrial applications. Viatec Recovery Systems of Richland, Washington, has licensed the technology and three commercial orders have been placed.

\section{Ion Exchange Membrane Process} removes cesium, strontium, and technetium from nuclear waste. A fully radioactive demonstration was completed at the Idaho National Engineering Laboratory in 1994. It is currently being tested for implementation at West Valley to remove technetium from liquid waste.

BetaScint Detector is an optical fiberbased sensor that monitors, characterizes, and quantifies uranium-238 and strontium-90 contamination in surface soils and similar media within minutes with no need for soil disturbance, collection, or transport. This detector system is being demonstrated at multiple sites and is being implemented in several operations.

Unsaturated Flow Apparatus (UFA) directly measures the subsurface migration of water, volatile organic compounds, and other contaminants in porous media. Within a few hours (compared to months or years using soil columns), it provides contaminant transport information. The UFA technology employs open-flow, ultracentrifugation with precision fluid flow. This equipment is available through Beckman Instruments and is the basis of a new testing service company started near Hanford.

Mediated Electrolytic Oxidation is an alternative to incineration for combustible low-level mixed waste forms and waste streams. This technology uses low-temperature oxidants in an electrochemical cell to oxidize hazardous organics. The technology has been licensed and is targeted for use at several sites.
Leveraging DOE-EM Technology Development through Related Laboratory Programs An important aspect of the National Technology Development Program is being able to leverage or build from activities in programs for other parts of DOE and other federal agencies. Staff responsible for the Laboratory's Technology Development Program also track the work for SERDP, LDRD, other DOEEM offices, and EPA's Environmental Technology Initiative which is applicable to Focus Area needs. These staff are also responsible for coordinating the Laboratory's international environmental technology activities. Results from this coordination effort will be provided to EM-50's International Technology Exchange Program and are expected to lead to new international outreach activities or partnerships.

Leveraging with these programs is beneficial but is relatively small compared to the benefits of leveraging with the Laboratory's fundamental research activities. Specifically, the Laboratory is building an Integrated Waste Processing Program, which will be the mechanism for linking a significant portion of research into DOE-EM's technology activities. The Laboratory is leveraging programmatic funding from both DOE-EM and DOE-ER with internal Laboratory Directed Research and Development funds to build capability and seed activities bridging the gulf separating fundamental research supported by DOE-ER and the highly directed work supported by DOE-EM. Collectively, these activities will form the core of an advanced waste remediation program to examine the waste remediation problem outside of any prescribed baseline.

Starting with fundamental science, we are leveraging DOE-ER funded programs, such as the Physics and Chemistry of Ceramic Surfaces, Surface Structure and Reactivity of Carbonate Minerals, and Molecular Theory and Modeling, and Chemical Structure and Dynamics Programs to develop fundamental, molecular-level understanding of the chemical and physical phenomena important to tank waste remediation. For the future, a range of fundamental research programs is being developed to address environmental management issues. These programs will be part of the EMSL.

At the opposite end of the technology development spectrum is the process science and engineering work supported by DOE-EM. For tank waste remediation, this work has been funded by DOE's Office of Waste Management through the Tank Waste Remediation System (TWRS). Changes in the DOE-EM approaches to managing tank waste remediation are expected to impact the Laboratory science and engineering programs. However, the need for fundamental understanding of the scientific and technical issues remains. Most work is directed at supporting the technical baseline and its enhancements. There are, however, efforts which are not explicitly tied to baseline technologies. These are aimed at providing the fundamental technical information necessary to make scientifically sound and technically defensible decisions regarding tank waste processing. One example in waste pretreatment is the Tank Waste Treatment Science task that is examining the fundamental chemical and physio-chemical behavior of the waste sludges and liquid supernatants, the separations materials used to treat the wastes, and the interactions between waste species and separations materials.

The last piece involves the development of programs that bridge the gulf between the fundamental and process science and engineering researchers. The Laboratory is investing discretionary funds in seed programs taking fundamental research ideas and concepts and transforming them into viable process science and engineering activities. Here there are three primary activities: the Advanced Processing program of the EMSL, the Advanced Processing Technology initiative, and the Tank Waste Remediation Advanced Technology Development program.

Key core capabilities being developed in the EMSL Advanced Processing 
Program that crosscut these program elements include colloid chemistry, experimental and computational fluid dynamics, catalysis, chemical and surface analysis, reaction chemistry, analytical chemistry, and process control. Current research of the Advanced Processing Technology initiative is focused in advanced processes and advanced materials. Advanced processes include efforts in separations, conversion, and fluid dynamics. These efforts are strongly coupled with the emerging efforts in the EMSL's Advanced Processing program and are developing linkages to the Advanced Technology Development, Tank Waste Treatment Science, and sludge processing activities. Finally, there is the Tank Waste Remediation Advanced Technology Development program, previously described.

\section{Management of Technology Imple-} mentation Since implementation is such a critical part of the Laboratory's Technology Development Program, the Laboratory has leveraged its DOE funds with internal funds to promote or manage the implementation process locally and on the national level. The Laboratory is actively involved in meeting the technology integration needs of EM-50's National Program and the specific needs of the activities at Hanford through the Hanford Technology Integration Program (HTIP).

The objective of HTIP is to identify and build links with our EM-30 and EM-40 technology users, industry, universities, and other units of government to develop, adapt, deploy to DOE sites, and commercialize new, federally funded and cost-shared technologies that have applicability to the special needs of the DOE complex and the broader needs of the private sector. Pacific Northwest National Laboratory has built relationships with both EM-30 and EM-40 technology users and initiated negotiations with numerous industries, firms, regional businesses, and units of government on behalf of the DOE-EM program. More than 2 years ago, HTIP took on the responsibility for working with the National Technology Development Program elements coordinated at Hanford to develop industrial partnerships and achieve commercialization in $22 \mathrm{prob}$ lem areas requiring innovative new technologies. During this time, partnerships with industry have been achieved in virtually all cases attempted (22) and, more significantly, roughly onefourth of these technologies have been deployed for use on DOE sites and/or made available to the commercial market for use by others engaged in environmental cleanup. Over the next 5 years, efforts will be focused on finding DOE sites on which to deploy these technologies and simultaneously pushing them to commercialization in the broader domestic and foreign marketplaces.

Key to the Laboratory's success is its approach to finding the "right" industrial partners capable of manufacturing and/or applying the technology, identifying the most likely site-specific EM-30 and EM-40 users of the technology, and bringing them together with the technology developer in what have come to be called, "one-onone-on-one" regional environmental interchange meetings constructing effective partnerships. Holding interchange meetings with industry to inform them of needs and opportunities available for participation is the first stage of this process. To date, six highly successful interchange meetings have been held and a network of some 900 environmental firms established in the Northwest.

Pacific Northwest National Laboratory proposes to expand its efforts to link DOE and commercial users of EM-50 technologies into effective relationships with the developers and their costsharing industry and university partners to ensure a more rapid penetration of the technology into both the DOE complex and the broader commercial marketplace. Key to its success will be combining on-going efforts to search U.S. and foreign markets for technologies (and technology needs) with its continuing mission of integrating the EM-30 and EM-40 needs of the sites with the technology developers and their industrial, university, and laboratory partners to create maximum opportunities to both deploy (to DOE sites) and commercialize (to domestic and foreign markets) those technologies. In this manner, the Laboratory hopes to provide critical support to the efforts of the STCG at Hanford as it pursues its task of linking site needs with available and developing technologies to speed cleanup.

In support of EM-50 goals, we are in the process of identifying and cataloging all of the environmental technologies available or under development at the Laboratory. This catalog or portfolio of technologies will give us a single source of information for identifying the "best" technology for meeting a customer's needs. Other uses of the technology profiles will be for press releases, public workshops, and technology transfer activities. The information contained in these profiles will be used to populate a nationwide technology database being managed by the National Technology Development Program.

\section{Application of Environmental Technologies}

\section{DOE-EM's National Technology} Development Program will be generating a large number of new technologies for application at DOE and other sites. In addition, domestic and international environmental industries will also be bringing new technologies into the marketplace. However, in most cases these technologies will need to be adapted for application in actual operations. Pacific Northwest National Laboratory is, and will continue, supporting the adaptation of new technologies as well as providing scientific and planning support to environmental operations at Hanford primarily, but also elsewhere in DOE and for other federal and commercial organizations.

The scope of work includes assessments of technical options; re-engineering, adaptation, and demonstration of technologies; development of scientific information to resolve operational issues, such as tank safety questions; economic, regulatory, and institutional analyses and management approaches; and systems engineering and risk management to support 
management decisions. The Laboratory is positioned to provide this support because of its broad technical capabilities, which are linked to a scientific support system and to experienced policy specialists; its experience as a long-term member of the Hanford team; its unique working relationships with industry and other partners; and its objective view of operational activities.

Several of the current special management initiatives supporting Hanford operations are described in the following paragraphs. The balance of this section describes major programs supporting the local and national needs of the Offices of Environmental Management. Several of the Laboratory's activities funded by these offices which support specific Hanford Site operations, such as facility operations and environmental oversight are addressed in the section on Hanford Site Support.

Stakeholder Participation at Hanford. Pacific Northwest National Laboratory, along with Westinghouse Hanford Company, is providing strategic support to DOE-RL in the management of the Hanford Advisory Board (formed in January 1994), a regional group of citizens representing local governments, Indian nations, and environmental groups examining Hanford cleanup issues. The Laboratory also is providing primary support to the Health, Safety and Waste Management Committee, one of four issue committees formed by the Hanford Advisory Board. Finally, the Laboratory is supporting broadbased public involvement activities at the site through its participation on the Hanford Public Involvement Network (HPIN). The Laboratory's Public Involvement efforts have increased the visibility of DOE's efforts to clean up the site; the open sharing of information will continue to improve the credibility of the Department and the acceptability of proposed technologies.

Privatization. The Pacific Northwest National Laboratory is supporting DOE in the development and implementation of a new strategy for the cleanup of the Hanford tanks. The tank cleanup is part of a program called the Tank Waste Remediation System. The
Laboratory has assisted DOE in the formulation, feasibility analysis, and now the implementation of the new strategy. This strategy uses the principles of competition to stimulate innovations and achieve cost reductions in waste cleanup. Interactive facets of the cleanup program include regional and national stakeholder concerns, multiple regulatory agencies, public and private financing, contractual reform, and complex technical issues associated with retrieval and processing as well as the long-term potential for environmental release from a disposals system. To deal with this challenge, the Laboratory has assembled a multidisciplinary team from Westinghouse Hanford Company, Argonne National Laboratory, and Los Alamos National Laboratory as well as a number of private organizations. Recently, the Secretary of Energy decided to proceed with implementation of the strategy, and a draft request for proposals for the first phase has been issued. The Laboratory has been asked to continue and broaden its support of the Hanford TWRS as implementation of the new strategy proceeds.

The Laboratory expects a significant role in the continuing need for technical innovations and scientific insight to support the TWRS mission. Highly directed engineering support will be required by the private sector for disposal and by a research and development contractor for other portions of TWRS. Longer term research and development will be required to address issues such as difficult wastes and waste form performance. Such issues represent high risk to the government that the private sector cannot assume. The Laboratory's role will be in the identification and prioritization as well as support of these needs.

The new cleanup strategy is a phased approach and the present request for proposals is only for the first phase or group of wastes. Determination of the goals, objectives, and scope of subsequent phases of the cleanup will require the use of systems engineering and risk analyses and must be integrated with site-level systems planning.
The Laboratory anticipates providing this service to DOE.

Risk-Based Decision Making. Pacific Northwest National Laboratory is supporting DOE-RL in the development of concepts, tools, and a process that incorporates a sound risk information basis into site strategic decision making. This approach will employ the Laboratory's significant risk assessment and risk management capability in integrating existing and newly developed risk information to prioritize site activities and demonstrate how Hanford is addressing its key health and ecological risk issues. This effort will be linked with the systems engineering initiative and the risk activities we are conducting to support the national EM program.

Site-Level System Engineering. The intent of this initiative is to support DOE-RL in achieving its strategic goal to "manage Hanford cleanup as a project." When successful, systems engineering will provide an integrating structure that will connect all elements of Hanford's cleanup mission. It will enable DOE-RL to plan, manage, and operate the site as an integrated system or project, rather than as a collection of independent technical programs.

In order to support this perspective, the Laboratory is working with DOE-RL to develop and implement top-down integrated planning tools and processes that will meet both RL management needs and DOE-HQ information requirements. The Laboratory is utilizing its extensive capabilities in risk assessment, risk management, decision analysis, and systems engineering to develop these tools.

A key issue in the use of such tools is identification of gaps in the technology base related to mitigation of health and environmental impacts. In addition, the Laboratory uses these capabilities to examine current cleanup baselines to identify areas with substantial potential for reduction in cost, risk, or the time required for implementation of the technology. This information is then used to focus technology development efforts into areas with higher potential. 
Of particular importance here is our system analysis efforts in support of DOE-HQ and its links to the Hanford Site. The impact of a technology is often wider than a single site, although its initial development may be focused on problems at a specific site. We use our complex level analyses (for example, in support of the Programmatic Environmental Impact Statement for DOE-EM) to scale technology impacts beyond a single site. This provides the Laboratory and DOE an understanding of the full set of potential benefits derived from a technological innovation.

Hanford's technology planning efforts and decisions (from the lowest, most detailed program-specific to the highest Hanford-wide cleanup strategies) will help to minimize work duplication, technology gaps, and science and technology conflicts.

Environmental Restoration The Laboratory is providing technology evaluation, acquisition, adaptation, and demonstration services for Hanford Environmental Restoration and Decontamination and Decommissioning programs. The objective of this effort, conducted for the Environmental Restoration Contractor Team, is to link EM-40 user requirements to the development efforts of EM-50. The effort involves evaluating and "challenging" the technology baseline and suggesting new technology and innovative approaches for incorporation in environmental restoration and decontamination and decommissioning projects. Environmental restoration efforts are evaluated on an operable-unit by operable-unit basis to identify restoration requirements that can be met more effectively by new and emerging technologies and innovative approaches.

The overriding driver of the program is to provide alternative technologies and approaches that exceed program risk reduction and cost goals, and meet schedule milestones. To date, operableunits related to groundwater, soils, and decontamination and decommissioning have been evaluated for technology needs.

Identified technology needs will be met through acquisition, adaptation, and demonstration of new technology developed by EM- 50 and others. Technology acquisition involves partnering with developers throughout the federal agencies as well as industry and foreign sources. This synergistic approach stretches the value of funding agencies, encourages broad innovative application of technologies, and ensures that activities do not overlap and that modification of existing capabilities takes preference over new starts. Technology acquisition means development of new technology, transfer of the technology from the EM-50 organization, or adaptation of industrial knowledge.

Linkages are also established and maintained with other federal agencies that have similar environmental restoration and decontamination and decommissioning programs to share lessons learned, technical approaches and solutions, and to reduce redundancies in developing technologies required to meet common problems. This promotes development and exchange of technologies for common problems and leveraging of capabilities and resources.

The current program includes diverse programs such as advanced characterization capabilities, adaptation of commercially available borehole geophysical capabilities, application of the Hanford permanent isolation barrier system, application of the in situ vitrification process, and adaptation of several in situ treatment techniques to Hanford-specific problems. Although all programs are for site-specific requirements, DOE complex and industrial applications are also possible. These programs capitalize on the Laboratory's expertise in analytical chemistry, geosciences, materials and process science, and systems integration and engineering.

Pacific Northwest National Laboratory is also supporting the Environmental Restoration Contractor Team in numerous Tri-Party Agreement-driven actions. Examples include treatability studies to determine and select remediation technologies at field sites, and the evaluation of ecological and health risks at specific sites.

Laboratory resources have also been applied to non-DOE facilities. For example, the University of California at Davis operated the Laboratory for Energy Related Health Research for $\mathrm{DOE}$ that was used to investigate the effects of $x$-ray radiation, strontium-90, and radium-226 on dogs. Pacific Northwest National Laboratory has supported a project to assess the contamination, decontaminate and decommission facilities, remove and dispose of radioactive sludge and a cobalt- 60 source, remediate groundwater and soil, manage waste disposal, and verify cleanup of the site. This activity has been a model for other decontamination and decommissioning activities.

Waste Management Technology Programs The purpose of the Waste Management and Technology (WM) Program is to support the broad objectives of DOE's Environmental Management Program with a specific focus on waste management support for cleanup and restoration of the Hanford Site. The principal purpose of the WM Program is to provide innovative, environmentally appropriate, timely, and cost-effective solutions to waste management problems of Hanford, DOE, other federal agencies, and the private sector.

The Pacific Northwest National Laboratory WM Program encompasses management of high- and low-level radioactive waste, hazardous waste, and mixed radioactive and hazardous wastes in all physical forms and from all sources. Laboratory technical organizations and staff involved in the WM Program have substantial expertise and significant current business in the principal phases of waste management operations including characterization, retrieval, treatment, transportation, storage, recycling, and disposal. Although the Laboratory's expertise is broadly applicable to all phases of WM operations, development and deployment of waste treatment technology is the core capability of the WM Program. Maintenance, enhancement, and commercial application of this core capability is central to our plans for future development.

The WM Program is organized into five principal elements: 1) Hanford solid waste management support; 2) the Spent Nuclear Fuel Program; 3) TWRS and other waste management science 
and technology support, including work order-funded projects and tasks from Westinghouse Hanford Company; 4) waste management support to DOE-HQ, other DOE sites, and other federal agencies; and 5) beneficial use of radioisotopes.

Hanford Solid Waste Management Support Pacific Northwest National Laboratory provides planning and science and technology support to the Hanford solid waste management programs. This support currently is combined within a single, integrated Solid Waste Technical Support (SWTS) Project to centralize management functions and increase synergies among the various tasks. Planning and management support include identification, quantification, and classification of future solid waste streams that will require management at Hanford. The Laboratory also maintains logistics modeling capabilities that are used in conjunction with waste inventory and forecast information to develop detailed estimates of future solid waste flows through the system. These capabilities, in conjunction with other analytical methods, provide the basis to analyze future solid waste storage, treatment, and disposal scenarios, and to evaluate feasibilities and impacts and determine preferred management options. Planning support is coordinated with Hanford mission planning and Hanford systems engineering activities to ensure compatibility among the various efforts.

Recent science and technology activities in the SWTS Project have included developing methods for determining radionuclide transport through soils under unsaturated conditions, measuring corrosion rates for waste container materials under typical storage and disposal conditions, and developing methods for quickly and accurately detecting and measuring corrosioninduced or mechanical flaws in solid waste containers.

\section{Spent Nuclear Fuel Program Support}

Pacific Northwest National Laboratory supports the DOE National, Hanford, Idaho Falls, and Oak Ridge Spent Nuclear Fuel Programs which deal with management of spent nuclear fuel, special nuclear materials, and associated storage facilities left from past defense materials production programs across the DOE complex.

For the national program, based on capabilities and experience, Laboratory scientists obtained assignments on all four national spent fuel management program technical committees. The Laboratory works with DOE-RL, Westinghouse Hanford Company, and DOE-HQ in development of the national program, currently managed by the Idaho National Engineering Laboratory. Pacific Northwest National Laboratory participants have also contributed significantly to the Technology Integration Committee and the Dry Waste Demonstration Committee. Support to these activities is expected to continue through FY 1996 and FY 1997. The Laboratory was assigned responsibility to prepare the Hanford section of the Spent Nuclear Fuel Programmatic Environmental Impact Statement. Recently our characterization staff have begun to support the development of characterization strategies for ORNL via funding from the national program.

In addition to supporting the national programmatic environmental impact statement process, we are also supporting the National Environmental Policy Act (NEPA) process for the Hanford Spent Nuclear Fuel Project.

The Laboratory worked with Westinghouse Hanford Company in FY 1993, FY 1994, and FY 1995 to develop plans for the Hanford spent fuel characterization program. In the spent fuel characterization project, we will work with Westinghouse Hanford Company to retrieve samples of fuel from the $\mathrm{K}$ basins for examination in the 327 Building fuel examination cells. Early emphasis will be on obtaining data to support interim storage in the pools to mitigate potential safety and operational problems.

In addition, the Laboratory manages the Applied Technology Program Office (ATPO) for Westinghouse Hanford Company to independently coordinate assessment and/or development of appropriate technology for the newly established and expanded Hanford Spent Nuclear Fuel Project (SNFP). The ATPO will be responsible for developing a process verification and test program that addresses options for fuel stabilization, waste forms, and long-term storage and disposal.

Pacific Northwest National Laboratory will also provide technical support in evaluation of structural integrity, leakage, and safety issues concerning the $\mathrm{K}$ basin storage pools. These technical efforts are expected to support principally the SNFP engineering and operations organizations.

The level of activity in characterization and environmental impact statement programs is expected to be stable for the next several years. Technical support for applied technology development, process development, waste form development, and engineering/ operations for the Hanford SNFP is expected to grow significantly over the next few years.

\section{Hanford Tank Waste Remediation System and Technical Development} Pacific Northwest National Laboratory assumed an expanded role in supporting the cleanup of Hanford tanks on October 1, 1993. The TWRS Integrating Contractor (Westinghouse Hanford Company) named the Laboratory to manage all technology development work for the program.

Major Laboratory efforts have concentrated on 1) establishing a technology development program with increased national involvement, building on recognized science and engineering capabilities at other DOE sites, within industry, and in universities; 2) ensuring that existing and planned technology development activities are directly linked with the systems-based requirements of the renegotiated Tri-Party Agreement; and 3) conducting public meetings with stakeholders to involve the affected groups in technology development planning, decision making, and execution.

To specifically increase national involvement, the Laboratory created the Technology Development Program Office (TDPO) with representatives 
from every national laboratory, the chemical industry consortium, and the university community to develop and direct the necessary TWRS technology development activities. All major FY 1994 technical products were delivered on schedule and within budget. Pacific Northwest National Laboratory provided TWRS with technology advancements that will have significant effects by drastically reducing costs for characterization (demonstrated the laser ablation/mass spectrometer screening analysis system on actual hot waste samples); greatly reducing high-level waste glass volume (demonstrated twice the flow sheet concentration of simulated waste sludges in borosilicate glass [50 percent versus 25 percent] in runs with the small-scale high-temperature melter); and enabling safe pipeline transport of radioactive slurries (developed an ultrasonic instrument which provides real-time measurements of the concentration of suspended solids in tanks and pipelines). In the downselection of low-level waste melter concepts, Pacific Northwest National Laboratory developed the process, chaired the technical panel, and passed on a ranking and recommendations of proposals from 16 industrial organizations to the Source Evaluation Board. Laboratory discretionary funds were used to provide in-depth understanding of sludge constituents and their rates of dissolution and provide DOEHQ with a document, $A$ Systematic Look at TWRS Privatization, pointed toward a more rapid initiation of remediation and lower costs as the result of competition in the private sector. Other internal investments focused on three strategic, high-impact technical areas-Tank Waste Properties, Process Control, and Waste Forms-with linkages established with the Advanced Processing Technology initiative and the Environmental Molecular Sciences Laboratory to build fundamental research capabilities.

Responsibility for the TDPO activities were transferred to the national Tank Focus Area during the second quarter of FY 1995. The responsibilities of the focus area are detailed in the Development of Environmental Technologies section. For FY 1995, Pacific Northwest National Laboratory provided technical support and continued technology development for TWRS in several critical areas

- tank waste safety issues

- improved/upgraded tank farm operation

- waste characterization

- waste retrieval

- waste pretreatment

- vitrification of the high-level waste portion

- vitrification of the low-level waste fraction

- privatization of tank waste treatment and immobilization.

The area of safety support is the highest priority within the program, with the Laboratory focusing on the resolution of key safety problems, such as the potential for flammable gas concentrations, explosive mixtures of ferrocyanide, runaway organic-nitrate chemical reactions, overheating of tank contents, and the release of toxic/hazardous gases to work spaces. In waste characterization, the focus is on the development of enhanced sampling and analysis capabilities. In retrieval, enhanced sluicing, subsurface barriers, and alternate technologies are being developed. Pretreatment emphasis is on solid/liquid separation, cesium/ strontium/technetium removal, organic destruction/thermal reconstitution, in-tank washing/leaching/caustic washing, and sludge processing. The vitrification focus is on glass formulation, product package evaluation, melter evaluation, feed and process refinement, and process/product development.

We continued to support DOE in the evaluation of the use of the private sector in the remediation of Hanford's high-level waste tanks. Evaluations to date have shown this to be a feasible alternative with the potential of reducing budget outlays and accelerating the progress on tank cleanup. Assuming that the privatization initiative goes forward during the last half of
FY 1995, the Laboratory's support to the TWRS program changes drastically. The Laboratory will continue activities in support of safety, characterization, and retrieval. We will also provide technical support to DOE during development of the request for proposals for privatization. It is anticipated that we will also continue some technology related activities in the areas of treatment and immobilization to reduce the risks associated with these activities. The extent of this support is not known at this time.

For FY 1996, the Laboratory will continue to provide technical support and technology development to the TWRS program in the areas of safety, characterization, retrieval, and immobilized waste storage. The Laboratory will also provide support to DOE in the execution of the privatized contracts for waste treatment and immobilization. As required, we may also provide technical data directly to the privatization contractors to support design decisions.

\section{Waste Management Support to DOE-HQ, Other DOE Sites, and Federal} Agencies In addition to collaboration with the Idaho National Engineering Laboratory mentioned above in the Spent Nuclear Fuel Program, for the past decade Pacific Northwest National Laboratory has provided technical support to the West Valley Vitrification Plant development. This activity is expected to diminish over the next few years as the plant comes on line. The Laboratory also has been a major contributor and participant in the EM-30 efforts to develop criteria and waste stream data needed to implement provisions of the Federal Facility Compliance Act (FFCA). Although our support to the development of the FFCA plans is winding down, implementation of FFCA provisions and other WM support could be areas of growth for the Laboratory at other DOE sites. As mentioned in the spent nuclear fuel section, recently Laboratory characterization staff have begun to support the development of characterization strategies for Oak Ridge National Laboratory via funding from the national program. 
Beneficial Use of Radioisotopes The widespread, pervasive use of radionuclides and radioactive materials in modern society is a phenomenal success story; but one that is generally not widely recognized. Radioactive materials can perform many tasks better, easier, quicker, and cheaper than alternative methods; have no substitutes in many applications; and are used extensively in all areas of industry, science, medicine, agriculture, and government. It is anticipated that the demand for isotopes will increase substantially in the future. Hanford is currently developing a business plan evaluating its large supply of valuable isotopes left over from its nearly five decades of service to the nation's military defense mission. The first phase of the business plan is focusing on medical and agricultural uses of existing Hanford stockpiles of isotopes. One specific application includes the coupling of certain isotopes to monoclonal antibodies for cancer treatment from inside the human body where direct tumor exposure is possible without damage to healthy tissue. Another example is the irradiation of seeds to destroy harmful pests prior to planting. The packaging and sale of these isotopes for socially useful purposes will, in parallel, have a beneficial effect on the long-range Hanford cleanup program by reducing the total inventory of waste materials on the Hanford Site and the associated cost of disposal.

\section{Hanford Site Support}

DOE-RL has assigned responsibility to Pacific Northwest National Laboratory for the safe and efficient operation of several critical research facilities and for environmental surveillance and oversight activities supporting the entire Hanford Site. These include surface and groundwater surveillance, meteorology and climatology, wildlife resources monitoring, cultural resource protection, and dosimetry coordination; preparation of major environmental documents, including environmental impact statements; occupational dosimetry; operation of the whole-body counter; radiological calibrations; occupational radiation exposure records; and the Hanford Technical Library. The Laboratory provides significant support to Hanford and its environmental restoration and waste management businesses, as described under the Application of Environmental Technologies section. The Laboratory will continue to seek well-recognized research staff from universities to participate in Hanford environmental programs and to initiate and coordinate peer reviews of these programs by nationally recognized experts. Funding for Hanford Site support activities are included in the appropriate sections of the Resource Projections.

\section{Pacific Northwest National Laboratory} Operational Compliance Program This program element has overall responsibility for planning, budgeting, and reporting on facilities operations ensuring safe and compliant facilities operations and management of wastes generated in the Laboratory's research and development and site cleanup support activities. Included in this element are facility development, systems upgrades, environmental compliance oversight, surveillance, and maintenance. Rapidly changing regulatory requirements continue to result in increasingly demanding operational safety and environmental compliance requirements that provide a major challenge in the face of escalating budget constraints.

Because of these changing requirements and the critical nature of the activities in these facilities, the Laboratory has implemented a comprehensive, integrated Environment, Safety, and Health (ES\&H)/Conduct of Operations management organization. This organization will implement an operational improvement program and will be responding to a set of performance based incentives.

Key activities include cleanup of Laboratory hot cells and related facilities to remediate safety concerns, and to renovate cells needed to support hot-sample analysis for TWRS, spent fuel characterization, and other future Hanford cleanup projects.
Environmental Sustainability Project In 1993, the Laboratory established its Environmental Sustainability Project (ESP) to help bridge Pollution Prevention (P2) efforts in the Laboratory's own operations with Pollution Prevention services provided for others. In 1994 these efforts were significantly expanded with the establishment of the P2 Info Research Center. P2 Info is a service provided by the Laboratory to the entire DOE complex to provide quick access to up-to-date information on a variety of Pollution Prevention topics. A key function of P2 Info is to be the primary vehicle for updating information in the automated portion of the DOE Pollution Prevention information infrastructure, the Energy Pollution Prevention Information Clearing House (EPIC). Also in 1994, we established a national DOE demonstration program in Microscale Chemical Analysis. By drastically reducing sample size, microscale chemistry techniques can dramatically reduce waste generated in the laboratory. Beginning in 1995 the microscale technique demonstrated at the Laboratory was be disseminated broadly to other DOE facilities. Also beginning in 1995, information concerning ESP initiatives was provided to DOE and the private sector through the ESP Internet Home Page. Individuals who have access to the Internet are able to connect with the ESP Home Page and view the entire ESP program with supporting pictures. Users also have the capability to download the Laboratory's P2 Plan and other P2-related documents. Chemical exchange between researchers and research groups will be promoted heavily in an attempt to lower procurement and disposal costs of unwanted chemicals. Going beyond mere paper recycling, the Laboratory will recycle all types of materials (paper, glass, aluminum, plastic, cardboard, and newspaper/magazines) in many of its facilities.

Environmental Oversight Pacific Northwest National Laboratory provides support to Environmental Oversight via the following. 
Public Safety and Resource Protection The comprehensive environmental surveillance and oversight program for the Hanford Site that is assigned to the Laboratory is to be conducted independently from the management and operations and the environmental restoration contractors. This program includes

- characterizing and monitoring the radiological and nonradiological status of the environment

- assessing onsite and offsite environmental impacts of site activities, estimating corresponding radiological and nonradiological risks to the public, and predicting future impacts of known contaminant releases and planned activities

- identifying and recommending programs needed for timely and responsive action on present and future Hanford environmental issues

- preparing NEPA documentation for the site

- monitoring Hanford biological (fish and wildlife) and cultural resources

- supporting DOE-RL in public information efforts and in presentations to cognizant regulatory agencies on matters that pertain to these activities.

During 1994, the Laboratory prepared environmental monitoring plans; conducted environmental monitoring and surveillance; calculated the radiological dose to humans onsite and offsite and reported the results in the annual Hanford Site environmental report; and conducted programs to keep the public and local, state, and federal agencies informed about Hanford environmental activities. Environmental media sampled included air, surface and groundwater, soils and vegetation, fish, wildlife, and foodstuffs. Cultural and archaeological resources were also characterized.

Occupational Protection As part of Hanford's program to protect the health and safety of workers, the Laboratory provides a complete assessment of occupational radiation exposure to ensure compliance with DOE Orders and federal regulations. The Laboratory conducts a comprehensive program of occupational dosimetry, including assessment of internal and external dose, measurement of internally deposited radioactive material, calibration of monitoring devices, and documentation and reporting of this information. These programs strengthen our health physics research programs by providing the means for identifying problems and areas for improving radiation dosimetry and measurement.

\section{National Environmental Policy and}

Assistance Pacific Northwest National Laboratory continues to provide technical assistance to the NEPA office and to operations office personnel to better implement the requirements of NEPA for DOE's projects. The application of SEN-15-90 has reinforced the importance of NEPA compliance across the DOE complex and the need for early involvement of the NEPA office in developing complete and comprehensive NEPA documentation. This, in turn, has increased the Laboratory's scope and level of effort in providing support to this effort. The program staff work closely with other DOE offices to assist in ensuring that all applicable federal, state, and local regulations are adequately addressed in NEPA documentation and review.

Laboratory technical support to the NEPA office emphasizes reviews of impact analyses, impact mitigation proposals and plans, and reviews of technology and regulations applicable to NEPA compliance. The Laboratory also develops analysis and management tools and methodologies to help ensure that the NEPA process is conducted in a timely and consistent manner across the DOE complex.

\section{Office of Environmental Safety and Health}

Pacific Northwest National Laboratory's participation in programs conducted by the Office of Environment, Safety and Health are outlined below.

\section{Environment}

Under the Deputy Assistant Secretary for Environment, Pacific Northwest
National Laboratory supports the Office of Environmental Policy and Assistance and the Office of National Environmental Policy and Assistance.

The environmental compliance technical support project assists the Office of Environmental Policy and Assistance in fulfilling its oversight and technical assistance responsibilities to ensure that the DOE's facilities and operations comply with federal, state, and local environmental requirements and with the DOE's environmental orders and policies. The technical support provided by the Laboratory assists the DOE's compliance strategies and facilities compliance divisions and spans the following areas:

- compliance methodologies development and technical analysis support

- review of key DOE compliance and policy documents

- day-to-day compliance activities support.

Assistance will be provided in several ongoing areas to help ensure that environmental documents receive consistent reviews from a policy perspective and that compliance reporting and review is consistent by 1) developing a manual for review of RCRA and CERCLA documentation, 2) identifying ways that will enable the Office of Environmental Policy and Assistance to track key CERCLA and RCRA corrective action decision points and to monitor activities in this area, and 3 ) supporting the development of a standard framework for reporting compliance information across the DOE complex.

As required under DOE Order 5400.1 (General Environmental Protection Program), each DOE site submits an annual environmental report and this data is combined to provide a summary of total radiological emissions and doses from the DOE's facilities. The Laboratory is continuing to support the Office of Environmental Compliance in finalizing the 1994 Summary Report, preparing the draft 1995 Summary Report, and providing guidance to sites on preparing their annual site 
environmental reports. The Laboratory is continuing to support the Office of Environmental Policy and Assistance in preparing the draft 1990, 1991, 1992, and 1993 Summary Reports.

Pacific Northwest National Laboratory provides technical assistance to DOE in fulfilling its responsibilities for providing environmental guidance and policy information to the DOE complex. The technical support provided by the Laboratory assists the DOE's RCRA/CERCLA and Air, Water and Radiation Divisions, and spans the following four areas:

- development of innovative methods for presenting guidance

- review of key environmental legislation, regulations, and associated documents

- risk-based standards support

- environmental support and assistance.

A continuing Laboratory effort has been to develop methods for streamlining site characterization at DOE's remediation sites. This effort integrates an observational approach with an engineering approach grounded in data quality objectives. The resulting integration is applied to the CERCLA remedial investigation/feasibility study (RI/FS) process. The Laboratory has completed a report containing guidance on techniques for streamlining the RI/FS process. These techniques will be applied to the Office of Environmental Policy and Assistance RCRA corrective action guidance development effort.

Currently we are developing a training video on the use of RCRA corrective action and temporary units management. The Laboratory will continue to provide technical support for developing new and updated guidance and strategies. While a major emphasis will be on developing graphic approaches to environmental guidance packages, we will also continue to provide technical support for preparing guidance materials in alternative formats, such as handbooks, guidance manuals, video training, memoranda, information briefs, and other formats.
Pacific Northwest National Laboratory is supporting efforts by the Office of Environmental Policy and Assistance to team with other DOE offices, particularly DOE-EM, to develop implementation-orientated guidance. Teaming efforts include four pilot projects on the streamlining approach to environmental restoration at specific sites using actual site data/scenarios as a method for obtaining "real-life" validation of previously issued guidance. The Laboratory is also developing guidance on phased approaches to environmental restoration under CERCLA.

Pacific Northwest National Laboratory will assess future environmental trends and proposed legislation and regulations to assist the Office of Environmental Policy and Assistance in establishing its near- and long-term priorities. As part of this effort, we will help determine how these trends may affect present DOE program priorities, needs, and requirements.

The Laboratory is also providing technical support to the development and application of standards for environmental restoration, waste management, and decontamination and decommissioning. We are providing members of the DOE working group on risk-based standards with critical information for decisions regarding the development of new standards and the application of existing standards. Pending decisions by this working group, we will also support standards development. The Laboratory will continue to support DOE in defining where standards are needed and where existing standards need to be adapted, particularly in the areas of human health risk and ecological risk.

The Laboratory continues to provide membership to the DOE Peer Review Panel for the technical reviews of performance assessments of DOE lowlevel waste disposal facilities.

The Laboratory will support DOE in developing an integrated program in risk assessment, risk management, and risk communication. This program will facilitate the use and acceptance of risk-based standards developed by DOE and other federal agencies for DOE sites.

Technical assistance is also provided to the Office of Environmental Policy and Assistance in fulfilling its environmental protection responsibilities for DOE-controlled nuclear sites and facilities. Assistance is provided in three major areas: 1) environmental radioactivity and risk-based guidance support, 2) radioactive and mixed waste management regulation and guidance support, and 3) quick turnaround a assistance. As site cleanup activities at the DOE's nuclear sites proceed, the DOE must provide technically credible, consistent guidance that is compatible with other federal guidance and ensures public and environmental radiation protection. Pacific Northwest National Laboratory activities are designed to be responsive to this need.

\section{Worker Health and Safety}

The Deputy Assistant Secretary for Worker Health and Safety is responsible for managing and directing comprehensive programs to ensure nuclear and non-nuclear safety in all the DOE's activities. Pacific Northwest National Laboratory conducts the programs described in the following paragraphs.

\section{Worker Safety Training and Safety} Evaluations Support The Laboratory develops and presents worker safety training courses, centralized coordination of occupational safety and health training resources, and assistance in development of occupational safety and health training requirements and guidance. DOE has designated the Laboratory as a lead laboratory for assisting the DOE with enhancing the nonradiological worker safety training program. Laboratory staff have developed several training courses to introduce workers, supervisors, managers, and safety and health professionals to the OSHA requirements for safety in the workplace.

\section{Mentoring Support for DOE Operations} Office and DOE Contractor Pacific Northwest National Laboratory mentors DOE Operations personnel or DOE contractor personnel in worker health and safety functional areas such as 
issues management, conduct of operations and maintenance, facility site representative training and program development, unreviewed safety questions, hazardous waste operations and emergency response program development and training. The purpose of the mentoring program is to provide expert technical assistance to DOE Operations Office personnel and contractor personnel to strengthen existing programs or assist in the development of additional safety functional areas.

\section{Accident Investigation Review and} Guidance Development The Laboratory supports the Office of Performance Assessment through the review of significant safety Type A and B accident investigation board reports for technical adequacy and compliance with the DOE directives. This program also includes the development and issuance of accident investigation guidance based on field experience and lessons learned, and conduct of operational and special assessments.

\section{Technical Support for Chemical Risk} Studies and Chemical Safety Guidance A chemical safety vulnerability review of the DOE complex was conducted between February and July 1994 at the request of the Secretary of Energy. This review identified generic vulnerabilities and management weaknesses that were indicative of failures of safety management systems to adequately address chemical hazards throughout their life cycle. In response, the Management Response Plan for the Chemical Safety Vulnerability Working Group Report was developed to provide a coordinated set of corrective actions. This plan took advantage of ongoing activities under existing chemical safety initiatives. In FY 1995, the Technical Assistance for Risk and Safety Analysis, and Process Safety Management programs were integrated into the new Technical Assistance for DOE Chemical Safety Program. Under this program, the Laboratory is providing the technical support necessary to assist DOE, the DOE Action Team for Chemical Safety (ATCS), and DOE contractors to successfully implement the recommendations of the Management Response Plan. The ultimate goal of this program is to ensure that DOE has effective management systems in place to address the chemical vulnerabilities noted and thus ensure the safe use of hazardous chemicals in the DOE complex.

Hazardous Waste Operations and Emergency Response Pacific Northwest National Laboratory is providing support to the Office of Worker Health and Safety in the development of their Hazardous Waste Operations and Emergency Response manual and its field implementation. In addition, the Laboratory assisted in the development of the Manager's Hazardous Waste Operations and Emergency Response Guide and the Worker's Guide.

\section{Health}

The Deputy Assistant Secretary for Health develops and recommends policies and standards related to radiation protection, industrial hygiene, and occupational medicine for application in DOE's facilities. Pacific Northwest National Laboratory assists in efforts to implement policies and standards, to evaluate their impact, and to monitor for compliance. The Laboratory also assists in development of a comprehensive data resource on workers to ensure effectiveness of health protection programs. In accomplishing this work, the Laboratory conducts programs for both the Office of Health Physics and Industrial Hygiene Programs and the Office of Epidemiology and Health Surveillance.

The Laboratory is continuing to develop capabilities to support new programs in the Office of Health. The Laboratory is expanding its efforts in nonionizing radiation and is developing new capabilities to assist the DOE in providing comprehensive chemical protection for workers and members of the public. These efforts include monitoring worker exposure to chemicals and evaluating the outcome of such monitoring. This has been expanded through the initiative entitled, "Health Protection and Standards for Hazardous Chemicals," described above in the introduction to the Office of Environment, Safety and Health section.
Health Physics and Industrial Hygiene Programs The primary objectives of the Laboratory's health physics and industrial hygiene programs are to 1) develop guidance to support requirements and good practices in operational programs; 2) solve technology problems in radiation and other measurements; 3) develop performance criteria and methods for implementing laboratory accreditation programs for radiation protection measurements and provide technical support in the operation of such programs; 4) develop and operate a Secondary Calibration Laboratory for DOE; 5) provide technical support to address key national and international issues affecting DOE; 6) provide support and assistance through special studies; and 7) provide a lead-laboratory systems approach to meeting DOE's needs.

Currently, five ongoing health physics projects are conducted by the Laboratory

- Radiation Protection Guidance

- Worker Radiation Protection Technical Support

- Worker Dosimetry Accreditation

- Research and Development Worker Exposure Assessment

- Hazardous Waste Worker Exposure Assessment.

Radiation Protection Guidance. The Laboratory assists DOE in the development or revision of guidance to support the new radiation protection rule (10 CFR 835), DOE Orders, and the new DOE Radiological Control (RadCon) manual. These guides are designed to assist DOE sites in appropriate implementation of rules and guidance and to ensure quality and uniformity of practices. A new initiative will be direct technical assistance to support DOE operations.

Worker Radiation Protection Technical Support. The focus of this program element is on special technical studies associated with improving the capability and quality of current health physics programs, and assessing the 
expected impact of standards, regulations, administrative requirements, and engineering design actions on DOE's radiological control programs. The project supports the development of new initiatives and capabilities, provides for quick turnaround specialized support, and supports development of guidance for establishing uniform protection programs throughout DOE.

Worker Dosimetry Accreditation. This project provides a mechanism for the development of improved calibration and quality assurance with respect to radiation measurement technology. Resolving problems associated with measurements and methodologies requires a cooperative multilaboratory effort and field-based studies. Accordingly, a portion of project funds is used for the direct support of identified activities conducted at other DOE laboratories, private industry, and universities having the recognized and needed expertise. To assist in this effort, the Laboratory has developed a worldclass radiation calibration facility and is extending this to include calibration capability for quantities of interest in worker protection other than radiation. This calibration laboratory was recently accredited by the National Institute of Standards and Technology and is the only Secondary Calibration Laboratory within DOE.

Research and Development Worker Exposure Assessment. The Applied Research Project for Dosimetry and Measurement allows DOE to identify and seek solutions to new health physics initiatives, which significantly advance the state of the art of personnel dosimetry and measurement systems, while maintaining a cognizance of "real" radiation protection problems within the DOE complex. The Applied Research Project focuses on problems that affect the determination of personnel doses and on developing improved, near-term concepts.

The Laboratory is assisting DOE in the development of guidance for assessment of radiation exposures to hazardous waste workers. This includes development and integration of guidance into assessment methods for other

\section{FLC Award for Excellence in Technology Transfer}

Cooled, optically stimulated luminescence is a versatile radiation monitoring technology that uses light and cold air-rather than excessive heat-to process dosimeters. Heat processing results in the destruction of the radiation exposure information contained in the dosimeter, but with COSL, the dosimeter can be reread for future reference. Also, the cool process provides more options for dosimeter packaging.

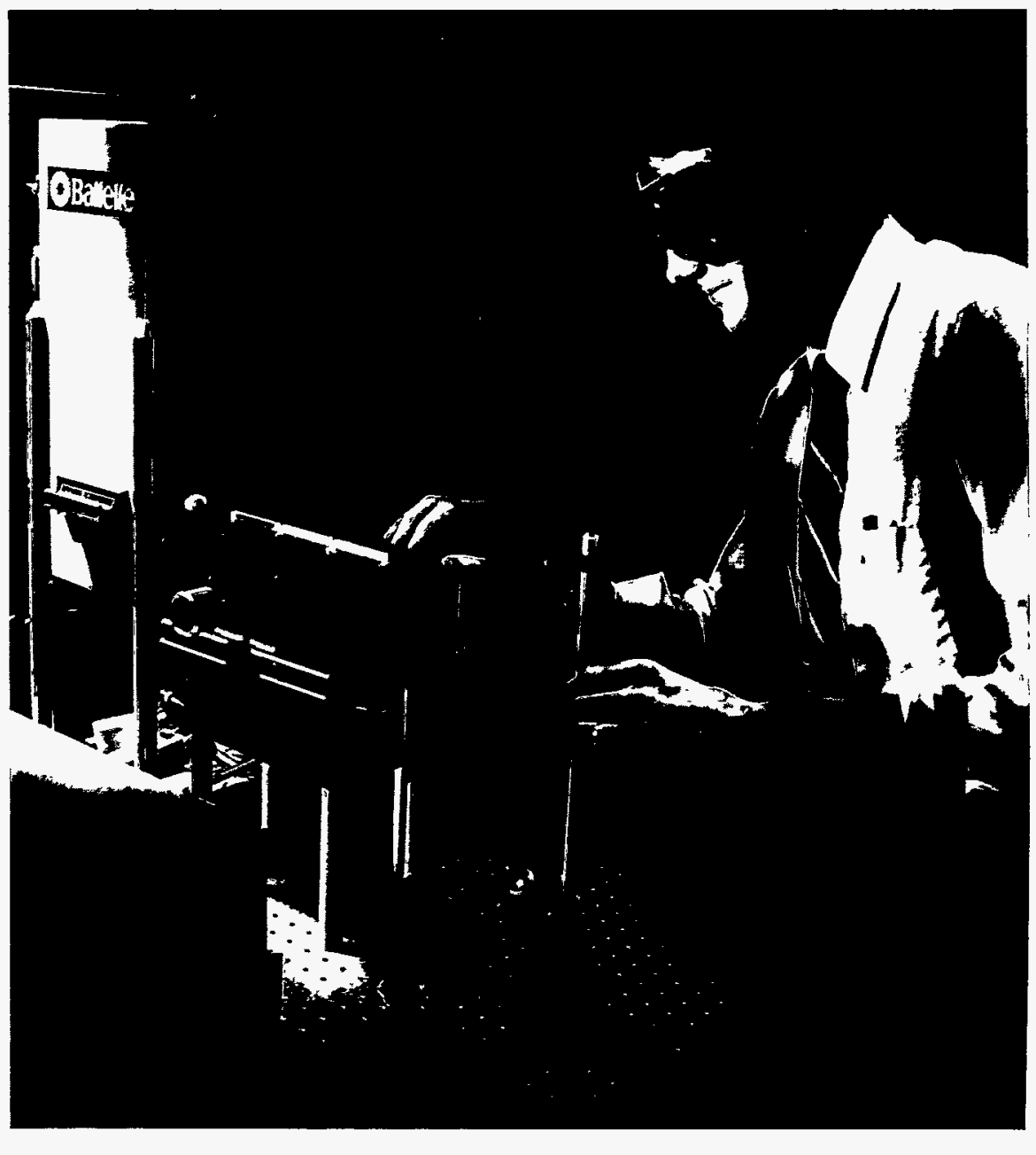

hazardous physical or chemical agents. In addition, the efforts involve database development and development of methods to correlate worker exposure with the task or job.

\section{Epidemiology and Health Surveillance} Two areas of technical support are currently provided by Pacific Northwest National Laboratory

- Technical Support for Epidemiology and Health Surveillance
- Technical Support for Comprehensive Epidemiologic Data Resource (CEDR).

Technical Support for Epidemiology and Health Surveillance. The Laboratory will continue to support DOE in collaborative efforts related to nuclear worker studies at the International Agency for Research on Cancer through coordinating a new collaborative study of workers in several 
countries. In addition, the Laboratory is conducting research on statistical methods for conducting epidemiologic dose-response analyses that account for errors and biases in estimates of external dose in nuclear worker studies.

Through other work, we will continue to emphasize molecular approaches to epidemiology and health surveillance. In particular, the Laboratory will focus on identifying biomarkers of exposure, disease, or susceptibility that are predictive of consequences of exposure to radiation and chemicals, and on understanding issues regarding application of these biomarkers to worker surveillance activities. A waste cleanup worker surveillance study with a biomarker component has been proposed for DOE sites.

Technical Support for Comprehensive Epidemiologic Data Resource. The CEDR program was established to develop a public-use database containing information about workers at DOE facilities. The Laboratory will continue to contribute to this program by providing data and documentation from studies of the health and mortality of workers at the Hanford Site and of their offspring, by expanding and documenting the Hanford worker database, by participating in the CEDR Provider Working Group in the areas of both data management and dosimetry, and by assisting in providing support to CEDR users.

Health Protection Standards for Hazardous Chemicals Initiative DOE sites share the problem of hazardous chemicals that have been disposed of improperly with many industries in the United States. Basic toxicological information is available for many of these chemicals and this data has been used for hazard identification purposes. In some cases, the probability and character of the toxicological effect is so clear and the extent of the problem is small or highly localized such that cleanup is the only logical action. In other cases, the chemicals located in such sites prove to have little or no health or environmental impact such that cleanup is not required or justified. There exists a third category of chemicals in which a high degree of risk is indicated by extrapolation of available health effects information, but the quantitative risk assessments are in considerable doubt. If cleanup costs are high for such chemicals, it is very cost-effective to experimentally confirm the degree of hazard posed by such chemicals. In general, this type of chemical has low intrinsic toxicity and weak carcinogenic activity but the chemical has been widely used in large quantities (e.g., as a solvent). If these chemicals are also mobile in soils, they frequently drive cleanup operations. In some cases the costs of cleaning up these chemicals is quite high, in the range of tens of billions of dollars nationally. If such cleanup cannot be justified with some certainty, these problems compete unfairly with radionuclide and other chemical hazards of established importance.

A second major problem in cleanup of hazardous wastes is judging the hazards to workers in the field. Unlike radionuclide measurements, where dosimetry is limited to the measurement of a relative few particles or some specific effects of these particles, chemical exposures have to consider measurement of each individual chemical. However, once the magnitude and temporal variation of an exposure can be measured, the probability of an individual's health hazard can be estimated if appropriate toxicological information is available.

With these two problems in mind, the Health Protection Standards for Hazardous Chemicals Initiative was begun in FY 1993 and enhanced significantly in FY 1994. The activities in the first year were directed at evaluating existing data for several chemicals of importance to DOE to identify those chemicals that fit into the category described above. A substantial investment was also made in developing an exposure assessment system for volatile contaminants.

A decision process for prioritizing those chemicals for entry into a directed research program is outlined in a decision tree. To simplify presentation, the research approach has been restricted to four general activities that would be critical for assessing risks from carcinogenic chemicals. Relatively minor modifications in the specific research direction allow the same process to be applied to a variety of other chemical hazards such as developmental toxicities, effects on reproduction, and neurotoxicity. In all cases, research on identifying the mechanism of action, the metabolite(s) of the chemical that is responsible for producing the effect, and the development of comparative metabolic and pharmacokinetic factors that govern the delivery of the chemical to the active site is appropriate. The coupling of the mechanism of action to the production of disease in quantitative terms will drive the development of an appropriate risk assessment model on a case-by-case basis. This approach is consistent with the new risk-based methodology for prioritizing environmental hazards that has been articulated by the EPA.

Efforts in the exposure assessment methodology has focused on the development of an instrument that can be applied to workers exposed to volatile chemicals. The methodology employs a specially designed apparatus for sampling expired air that allows it to be directly introduced into a portable mass spectrometer. This arrangement allows for continuous monitoring of chemicals in the expired air. Applying a pharmacokinetic model to these data allows estimation of the magnitude of the individual's last exposure. In combination with analysis of body fluids (e.g., metabolites in urine) or badge dosimeters, the total exposure in a particular work period could be estimated. The apparatus has the added advantage of detecting some products of lipid peroxidation in the expired air that are triggered by particular volatile chemicals such as carbon tetrachloride. This information provides a measure of potentially harmful reactions by which such are thought to produce their adverse effect.

\section{Independent Oversight}

The Pacific Northwest National Laboratory program for the Deputy Assistant Secretary for Independent Oversight involves assessing the status and condition of Environment, Safety, Health and Safeguards and Security programs at all major DOE facilities by making 
field inspections, evaluating trends and problems in specific areas, and reporting to DOE management on the conditions. The Laboratory will continue to provide direct technical and field support to this program by 1) participating in inspections and evaluations of field operations offices, 2) analyzing survey results and performing trend analyses, 3) preparing summary reports on findings, and 4) preparing field guides to assist the DOE and field inspectors in performing their duties. The Laboratory will focus on developing new tools, including information management systems, and techniques that can be applied in the evaluation process.

\section{Energy Resources}

As a multiprogram laboratory, Pacific Northwest National Laboratory provides scientific knowledge and innovative technologies to many $\mathrm{DOE}$ programs directed toward achieving DOE goals, such as diversifying energy supply, using energy more efficiently, and reducing environmental impacts. The Laboratory is focusing on improving the utilization of existing energy assets and developing a new generation of distributed energy systems. This focus provides the framework for using our core competencies to contribute to near-term DOE goals that address the current national and international energy infrastructure. It also establishes the context for providing leadership in an emerging national need for new distributed energy systems that will be central to the revolution now under way in the U.S. energy industry. The Laboratory expects that the pressure to decentralize energy services will significantly influence U.S. energy policy and economic competitiveness in the 21 st century.

With the linkage between energy, the environment, and the economy, the Laboratory has focused its capabilities toward the following objectives:

- improve transmission, distribution, and utilization of electricity and gas

- improve energy efficiency and use in buildings
- reduce emissions and weight in motor vehicles

- reduce energy consumption and wastes produced in industrial processes

- support international agreements and facilitate energy safety and reliability improvements in the Former Soviet Union

- understand the technological and policy issues that impact energy policy and global environmental change

Pacific Northwest National Laboratory's scientific and technical activities in each DOE program contributing to the energy resources core business are described below. The descriptions also explain proposed new initiatives within these organizations.

\section{Office of Energy Efficiency and Renewable Energy}

Cost-effective, environmentally benign energy resources are critically important to the nation's economic productivity. In our efforts for the Assistant Secretary for Energy Efficiency and Renewable Energy, the Laboratory draws upon its three core competencies to develop and deploy efficient technologies that improve the efficiency of the buildings, transportation, and industrial sectors and increase the reliability and utilization of the nation's vast transmission, distribution, and storage system assets.

The DOE-EE role is expanding and changing to address significant new challenges resulting from the Climate Change Action Plan, the Energy Policy Act, and DOE's new strategic plan which emphasizes energy resources, international competitiveness, and environmental quality. The Laboratory plans to help DOE-EE respond to these new challenges both through current programs and through new initiatives. Through the Energy Technology Development initiative which was described previously, we are enhancing Laboratory capabilities to address emerging needs in three of the Department's core businesses (energy resources, economic productivity, and environmental quality as it relates to reducing the impacts of the energy system). We expect to continue to participate in developing collaborative efforts with DOE and industry which access the capabilities of the laboratory system.

New DOE-EE program initiatives are anticipated in areas that build upon these current efforts and also address key goals from DOE's environmental quality and economic productivity core businesses. Selected highlights include

- continued support (internally funded) to the industry-led distributed utilities project, which includes Electrical Power Research Institute, Pacific Gas and Electric, and the National Renewable Energy Laboratory

- expansion of programmatic support for science and engineering support of the utility transmission and distribution system. Support of electricity transmission and distribution research and development efforts will grow both with Bonneville Power Administration and with the Office of Utility Technologies. Pacific Northwest National Laboratory also anticipates broadening this activity to bridge to the natural gas transmission and distribution system as well.

- a new program to apply microtechnologies to building system technologies

- expansion of the Laboratory's participation in the USCAR initiative

- support planning and implementation of the Chemicals Industry of the Future and the Refinery of the Future initiatives

- leadership in developing the National Electric and Magnetic Field Research and Communication Program (established by EPAct)

- expansion of Laboratory support to DOE-EE's portion of the Climate Change Action Plan.

The projected funding for DOE-EE for the planning period is shown in the 
Resource Projections. During the period, the level of effort is expected to experience at most, modest growth, reflecting current budget realities.

\section{Office of Utility Technologies}

For the Office of Energy Management (EE-14), the Laboratory conducts research on potential biological effects of electromagnetic fields, energy storage technologies, wind resource characterization, and integrated resource planning; we lead the Management Analysis Program. The Laboratory intends to participate in the proposed multilaboratory collaborative efforts with the utility industry in areas where our competency can lead to a substantial contribution.

Electric Energy Systems (AK) The Laboratory will continue its basic research in electromagnetic field interactions on biological systems and in the identification of potential health effects in support of the National Institute of Environmental Health Sciences (NIEHS), as outlined in the National Electric and Magnetic Field Research and Communication Program. In addition, we will work with DOE and Oak Ridge National Laboratory to develop and implement a comprehensive electromagnetic field engineering program that includes electromagnetic field measurement and assessment and the development of mitigation strategies and materials.

The research effort currently under way for the Office of Energy Management includes comprehensive studies to assess possible biological effects of electric and magnetic fields related to the use of electric power. This research is designed to provide data to help set the maximum levels of exposure to those fields that are biologically acceptable for both workers and the general population. The research program also addresses the broader issues of increased electromagnetic fields in the environment from a variety of sources and their health implications for humans and animals.

Current program elements include investigation of possible links between cancer and exposure to electromagnetic fields, examination of neuronal and neuroendocrine involvement in response to exposure, and the study of basic cellular and genetic mechanisms of interaction. The work complements related Laboratory studies sponsored by the Electric Power Research Institute, Bonneville Power Administration, DOE, and the National Cancer Institute. It is expected that, as this program responsibility shifts to NIEHS, we will continue to conduct this core research. In addition, the Laboratory will employ its significant experience in public outreach to support the communications goals of the national program.

The National Electric and Magnetic Field Research and Communication Program, established by the Energy Policy Act of 1992, has a 5-year sunset provision. The Laboratory has been investing its discretionary resources for the past several years in the development of engineering systems deemed important for supporting the engineering requirements of the national program. The development included advanced exposure systems, electromagnetic field shielding materials, systems for characterizing the electromagnetic field signatures of electrical devices, and supporting technologies including computer "visioning" systems. Using these capabilities, the Laboratory will work with Oak Ridge National Laboratory and the Office of Utility Technologies to develop an engineering program that can meet the needs of the national program on a timely basis.

Under the Management Analysis Program, the Laboratory is providing strategic planning, stakeholder outreach, and systems analysis support to the Office of Energy Management. The focus of this program is to help the Office conduct utility-related research that is highly responsive to the stakeholder community, and that will result in reliable and cost-effective energy delivery systems. The objectives of this program are to support continuous two-way communications with the stakeholders for utility research, develop a clear understanding of industry research and development needs and to convert them into a long-term strategic plan, to develop metrics for evaluating the full range of the Office of Utility Technologies (and other) technologies, and conduct systems analyses to determine the appropriate roles for and the relative value of alternative energy storage and delivery technologies.

Energy Storage Systems (AL). The objective of our Energy Storage Program is to identify and develop economically attractive advanced thermal energy storage technologies that contribute significantly to more efficient utilization of energy, contribute to national competitiveness or improved environmental quality, and are beyond the scope of current privately sponsored research and development efforts. Budget uncertainty is likely to curtail this effort significantly in the near term. However, several new advanced thermal energy storage systems are still under development. In one concept, molten salts are used in coalfired plants to meet intermediate and peak loads. Use of molten salts and oils in cogeneration applications has been shown to be particularly attractive and is being investigated. Additionally, use of chill storage systems for gas turbine inlet cooling is also attractive, and co-funded efforts are now being initiated to develop this technology.

Efforts in seasonal energy storage are focused on understanding the geotechnical issues associated with storage of heat and chill in aquifers, and development and transfer of technology for aquifer chill storage to reduce summer peak electrical loads.

The Laboratory is also investigating the use of superconducting magnetic energy storage (SMES) for utility load management and system control, working cooperatively with organizations such as Bonneville Power Administration, Defense Nuclear Agency, utilities, and the Electric Power Research Institute to advance the nation's understanding of the potential benefits of this technology. Pacific Northwest National Laboratory will work with Sandia National Laboratories and the Office of Utility Technologies to help 
characterize the appropriate role for SMES vis-a-vis other electric and nonelectric storage technologies.

Wind Energy (EB) The Laboratory's major responsibilities in the Federal Wind Energy Program have centered on wind behavior research that is directly relevant to the development, deployment, and operation of costcompetitive wind energy conversion systems. This activity has included research on the turbulent wind encountered by a rotating blade as well as complex terrain and turbine wake influences on flow variability that affect siting and performance of wind turbine arrays.

In FY 1994 the Office of Utility Technologies made the decision to co-locate all wind energy research at the National Renewable Energy Laboratory. Pacific Northwest National Laboratory has worked with, and will continue to work with the Office of Utility Technologies and National Renewable Energy Laboratory to ensure a smooth transfer of the program with minimum lost momentum. We will continue to contribute to the DOE Wind Program as appropriate, seeking to utilize expertise in aerodynamics and electrical systems to assist industry in developing and sustaining international leadership in wind power technology development and implementation.

Integrated Resource Planning (EK) Integrated resource planning is an important vehicle for meeting the nation's future energy needs in a costeffective and environmentally responsible manner. The Laboratory's role in the program has concentrated on the performance of industrial demandside management measures and in working with utilities to structure effective approaches to demand-side management.

As Bonneville Power Administration has in the Northwest, other federal power marketing agencies are now planning to play an increasing role in the implementation of integrated resource planning. This trend is driven by a realization of the appropriateness of integrated resource planning and emphasized by the requirements imposed on power marketing agencies by the Energy Policy Act of 1992.

Pacific Northwest National Laboratory has vast experience in integrated resource planning and implementing it in the Northwest, New England, and in several countries of Eastern Europe. Implementation experience has been through the Laboratory's close working relationship with Bonneville Power Administration and other utilities in the Northwest and elsewhere. The Laboratory is working with DOE-EE to clarify where our unique capabilities can provide value, particularly in areas of regional energy planning using integrated resource planning approaches, demand-side management performance, industrial and load shaping demand-side management measures, and innovative marketing of demand-side management.

\section{Office of Building Technologies (EC)}

The Laboratory's Buildings Energy Program supports the Office of Building Technologies objective "to lead a national program to improve the energy efficiency of the nation's buildings and to increase their use of renewable resources." Our support includes research, development, and deployment activities designed to target areas with high-efficiency improvement potential that are not addressed by other organizations. The Laboratory seeks the active involvement of other government, industry, and private-sector organizations in planning and conducting program activities to ensure the rapid transfer and application of the knowledge and products developed.

\section{Economics and Systems Analysis} Program (ESAP) The Laboratory leads a multilaboratory collaborative activity to provide the Office of Building Technologies with a centralized point of contact for all planning and analysis activities. Major FY 1994-1995 activities included quantifying metrics of potential program performance, developing strategies for implementation of the Energy Policy Act and the Global Climate Change Action Plan, designing information systems to access buildings data, updating and revising buildings sector analysis tools, and analyzing emerging strategic issues in support of planning documents. A particular focus of our future work with the Office of Building Technologies will be continuously improving the data access, analysis, and decision-support systems available to the Office by bringing advanced information technologies to bear.

Building Systems Program Pacific Northwest National Laboratory will also support the Office of Building Technologies' new Advanced Buildings for 2005 Initiative through ongoing Office of Building Technologies work and internal investments. "Design for Best Practices," as authorized by EPAct Section 2104, directs $\mathrm{DOE}$ to initiate programs to combine existing energy guidelines/handbooks, energy analysis programs, and expert system design advisors and embed them into commercial software using Cooperative Research and Development Agreements (CRADAs), consortia, and industry collaboration. The developed products will provide guidance to building designers and decision makers for implementing energy technologies and integrated design strategies. A program element will also be initiated to integrate commissioning and building system operation technologies with design tools to ensure efficient, cost-effective operation.

The Laboratory's Building Systems Program develops knowledge and technologies to improve energy-related decisions made during all phases of a building's life cycle. Current efforts are carried out through the Design Tools (formerly Advanced Energy Design and Operation Technologies [AEDOT]) and Building Operation Research projects.

Software resulting from collaborative efforts with the private sector in the Design Tools project is putting analytic tools, artificial intelligence, and sophisticated computer graphics in the hands of architects and engineers who make decisions about energy efficiency as they design, construct, and operate buildings. The first AEDOT prototype, 
completed in a collaborative effort, demonstrates how a set of knowledgebased software tools can serve as a panel of consultants that automatically and continually evaluate a building design as it is developed by a designer and provide suggestions for improvement. A second collaborative project, with a private-sector firm and a university, has produced a software tool that integrates a heating and cooling loads calculation capability with a computeraided design system to encourage use of energy analysis as part of the design process. This software tool was first distributed in June 1995 as part of a widely used commercial computeraided design package. Efforts in FY 1996 will continue development of the fundamental energy design technology with enhancements that will build on the energy modules and will focus on offering enhanced capabilities to meet the needs of building designers and users of the software. In addition, efforts to add new members to the CRADA will be continued.

In the Building Operation Research project, a series of Building Operation workshops are planned to solicit input for DOE program planning. The goal of the workshops is to obtain privatesector involvement and to encourage better coordination among efforts throughout the nation that focus on developing and implementing ways of making the national building stock operate more efficiently. This effort will produce a program plan endorsed by important stakeholders, as well as initial projects in which "real players" from the private sector have.committed to participate.

Building Energy Standards Program (BESP) The Laboratory assists DOE with the development and implementation of building codes, standards, and guidelines to improve the energy efficiency of new residential and commercial buildings. These efforts involve partnerships with federal agencies, state and local governments, the building industry, building owners and developers, lending institutions, utilities, and public interest groups.

The standards, mandatory for federal buildings and voluntary for nonfederal buildings, continue to move from the development to the implementation arenas. In the coming year, the program will address new requirements in the 1992 Energy Policy Act, as well as continue ongoing development and implementation activities consistent with the Act. Major new activities will define the process for certifying state energy codes per Act requirements and support standards adoption efforts at the state and local levels. Efforts will continue to support standards development and issuance organizations with the promulgation of improved standards. The program will continue to work with federal agencies to adopt and implement improved standards. Technical support also will continue to the Department of Housing and Urban Development for the development and issuance of energy standards for manufactured housing and the Federal Loan Guarantee Program.

\section{Federal Energy Management Program} Pacific Northwest National Laboratory, along with the National Renewable Energy Laboratory, Lawrence Berkeley Laboratory, and Oak Ridge National Laboratory, are key participants in the Federal Energy Management Program (FEMP). The main goal of FEMP is to facilitate effective energy management across the federal sector. The Laboratory supports FEMP in the areas of information coordination, technical assistance, and new technology demonstrations as follows:

- developing analytic approaches and tools to support federal energy managers in the identification and implementation of specific projects at federal sites

- facilitating demonstration of new technologies at specific sites across the federal sector

- developing and presenting training courses on various analytic tools developed by FEMP

- coordinating (as requested) specific initiatives defined by the Office of Building Technologies or Congress.

The primary analytic tool that has been developed for FEMP, with funding coordination from the Army Corps of
Engineers Construction Engineering Research Laboratory, is the Federal Energy Decision System (FEDS) model that is an outgrowth of the federal energy decision screening process. The FEDS model provides a comprehensive approach to fuel-neutral energy resource planning and acquisition. Information obtained from the model can be used as the basis for negotiation of custom demand-side management programs with either the servicing utility or energy service contractor. The model has been applied at several specific locations and development is under way to increase the functionality of the model. In FY 1995, FEDS Version 3.0 was released for distribution to federal energy managers. This version is entirely Windows ${ }^{\mathrm{TM}}$-based and allows the user to input site-specific information to increase certainty in potential energy and cost savings from implementation of projects at the site.

The Laboratory also supports efforts to demonstrate, in partnership with equipment developers, utilities, trade associations, and other federal agencies, the reliability and cost-effectiveness of advanced energy technologies at various federal sites. Our efforts under FEMP are leveraged by Laboratory support for a similar program under the DOD Strategic Environmental Research and Development Program.

The first technology demonstration program (in FY 1992) evaluated the performance of two 15-ton naturalgas-powered rooftop air conditioners. Results from the demonstration program indicate that over the estimated 15 -year life of the equipment the net savings is estimated to be $\$ 120,000$.

Other new technology demonstration programs are in various stages of completion. They include a Seahorse gas hot water conversion system, naturalgas-engine-driven heating/cooling equipment for residential facilities, and a geothermal heat pump program. The Laboratory is currently in the solicitation process to develop a broader list of new leading edge technologies to be evaluated in future years, including thermal energy storage systems and high $R$ windows. 
Pacific Northwest National Laboratory also assists in the development and delivery of selected training programs for federal energy managers, including energy and water management, and operations and maintenance. During FY 1995, a total of 21 workshops were held: 11 FEDS, 8 Water Resource Management, and 2 operations and maintenance preview workshops.

In the future, the incorporation of water efficiency and environmental externalities into the decision and technology selection criteria will be an emerging issue. The current FEDS model may be expanded to provide the user with the ability to respond to issues regarding water resource management and water conservation. Issues related to energy savings verification and retention protocols will also be of interest.

Building Equipment The Laboratory is working with the Building Equipment Division to investigate the feasibility of applying microtechnologies to building equipment and systems. An initial effort involves use of microscale technology in heat pump systems to optimize design of the absorber and to control operation of absorption cycle heat pumps and chillers. FY 1995 investigations identified ion sensitive field-effect transistor (ISFIT) technology as being most appropriate for this application. A prototype ISFIT microscale ammonia concentration sensor is being fabricated and will be tested in FY 1996.

\section{Office of Industrial Technologies (ED)}

The Laboratory's role in supporting the Office of Industrial Technologies ranges from program planning and evaluation to technology development and more fundamental research. Current technology development programs draw upon our process science and engineering competency to recover energy from dilute industrial wastes, replace solvent cleaning with supercritical carbon dioxide, and develop alternative feedstocks. More fundamental efforts are directed toward developing computational chemistry and engineering tools for industry. All of these programs emphasize formal and/or

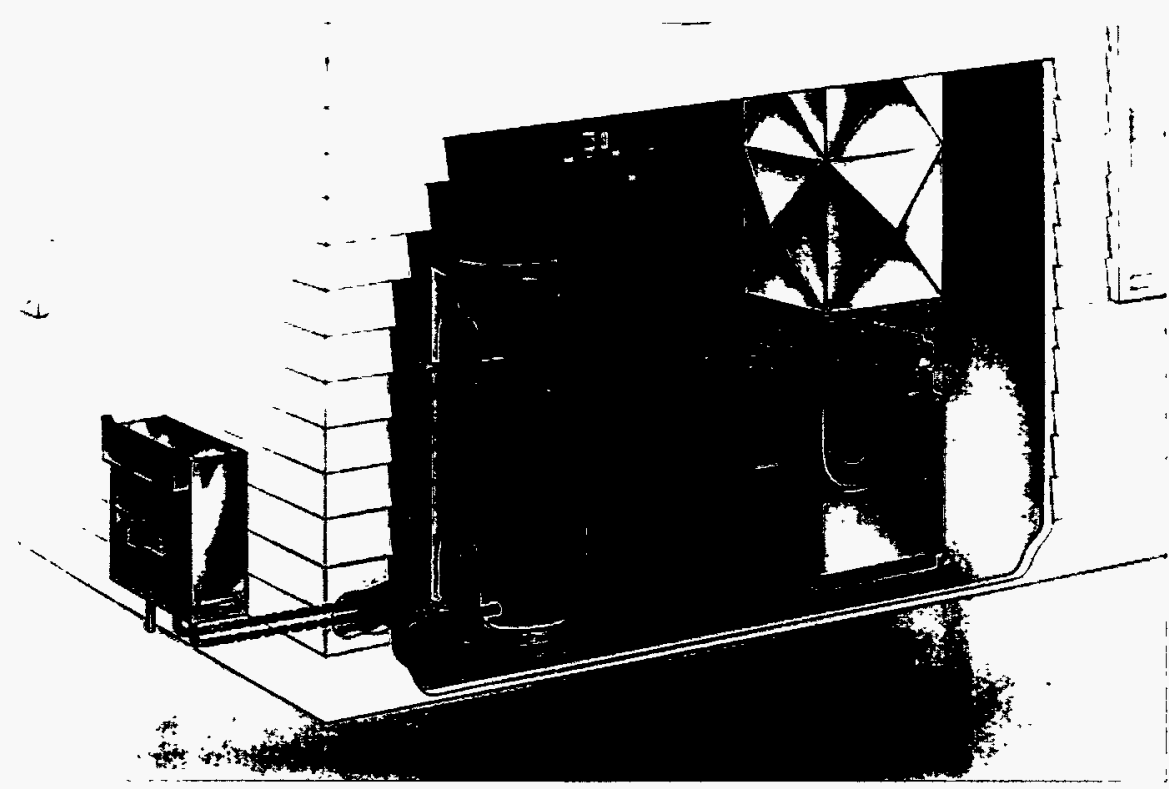

The Seahorse system "converts" a standard electric water heater into a natural-gas-fired system. The system consists of an externally mounted gas-fired water heater and a flow loop, which connects to an existing tank-type electric hot water heater. The electric elements on the existing tank are disconnected and the tank is used to store the water heated by the external gas-fired unit.

informal collaboration with the private sector, and all new programs and proposed initiatives are being conducted with partners across the laboratory system. For example, Pacific Northwest National Laboratory is supporting Argonne as it helps the Office of Industrial Technologies develop the Refinery of the Future initiative. In addition, the Laboratory is providing lead support to the Office of Industrial Technologies for developing a framework for its current and future chemicals-related programs that best meet industry's needs. The approach for this project is to engage chemical industry leadership in dialogue and to integrate input into the Office of Industrial Technologies' Industries of the Future initiatives. A first report, Developing a Chemical Industry Strategy: State-ofthe-Industry Profile, October 1994, overviews the industry and the driving forces shaping its future.

Industrial Waste Program Development of the Thermochemical Environmental Energy System (TEES®) technology continued this year. TEES uses a metal catalyst in a high-temperature, pressurized liquid water environment to convert wet organic industrial wastes to a useful fuel gas product and clean water. Current research and development efforts are directed at the use of a continuously fed bench-scale tubular reactor system to test catalyst effectiveness and stability with further tests to evaluate specific industrial applications in a scaled-up (half-ton per day) engineering development unit. During FY 1994 an Industrial Onsite Demonstration Unit, which operates at 60 gallons per day, was completed by the process licensee, Onsite* Ofsite, Inc. and is being operated by them under a CRADA with the Laboratory. The unit will be used through FY 1997 for technology demonstrations and site-specific tests of industrial applications of TEES.
Alternative Feedstocks Program
Pacific Northwest National Laboratory also participates in a joint effort with other national laboratories (Argonne National Laboratory, Oak Ridge National Laboratory, National Renew- able Energy Laboratory, and Idaho National Engineering Laboratory) to find renewable-based routes for chemi- cals to displace the use of petroleum. Both biochemical and thermochemical conversion routes are considered in the program. During FY 1994, we per- formed laboratory testing of catalytic processes to directly convert or to 
upgrade biomass-derived feedstocks into commodity chemicals. A strong industrial component is included in the program such as the use of CRADAs with industrial process developers and a program review panel of industrial experts. Scale-up of the processes in conjunction with the industrial partners is the goal of the program in FY 1995 and beyond.

Advanced Industrial Concepts Program The Laboratory also supports the Office of Industrial Technologies in precompetitive research and development to provide industry with the computational, analytical, and theoretical tools needed to make dramatic improvements in industrial energy and materials efficiency. In this vein, fundamental work aimed at the development of highly selective industrial catalysts is being carried out by the Laboratory staff in support of Office of Industrial Technologies Advanced Industrial Concepts Division. Staff at the Laboratory are also working to develop new computational models for predicting fluid behavior in industrial power systems. These models will permit more realistic modeling of fluid properties (such as viscosity, thermal conductivity, and density) and of bubble dynamics. Information gained from this research will be applied in the development of improved heating and cooling systems, as well as in industrial power plants.

Program Support Pacific Northwest National Laboratory plays an important role in providing technical planning and support for Office of Industrial Technologies research, development, demonstration, testing, and evaluation programs. This includes assisting the Office of Industrial Technologies in the strategic planning of its research and development programs and research initiatives, and supporting the Office of Industrial Technologies' technology transfer and tracking efforts.

Through the Industrial Energy and Environmental Analysis Program, the Laboratory is developing or evaluating technical, economic, or policy information regarding industrial energy use and related environmental impacts. Key activities for FY 1995-1996 include improving Office of Industrial Technologies use of industrial energy models and databases, evaluating energy efficiency data from the energy analysis and diagnostic centers, developing a computerized energy technology analysis data system, characterizing the energy and environmental features of advanced technologies from the Office of Industrial Technologies, assessing the economic productivity impacts of the Office of Industrial Technologies process technologies, and developing metrics for the economic and environmental impacts of advanced industrial energy and waste minimization technologies.

In support of the Office of Industrial Technologies' technology maturation and tracking program, the goals for 1995-1996 include identifying commercialization opportunities; developing collaborations with other federal and state agencies, private industry, utilities, and trade associations; developing seminars and training programs; and sponsoring in situ demonstrations of technologies. In addition, the Laboratory will support the Climate Change Action Plan as well as identify both primary and secondary impacts of the Office of Industrial Technologies' commercialized technologies.

\section{Office of Transportation Technologies (EE)}

Pacific Northwest National Laboratory is currently working with the Office of Transportation Technologies and the automotive industry to develop lightweight metals and reduce emissions. These programs are under the umbrella of the U.S. Council for Automotive Research (USCAR). The programs focus on advanced forming technologies for lightweight metals and composites. Primary emphasis will be on aluminum alloys. Additionally, programs on reduced emissions for internal combustion engines are being developed. These programs focus both on reducing hydrocarbon generation in the engine cylinders and on trapping and destruction of hydrocarbons coming from the engine.

The Laboratory continues an environmental support function with the
National Renewable Energy Laboratory jointly sponsored with Transportation (biofuels) and Utility (biomass power) funds. The support includes several task areas related to thermochemical biomass conversion. Laboratory analysis is being done on process effluents, and cleanup methods for the products are being developed, as required. Regulatory assessment is also an ongoing portion of this support. Processing tests to clean up products and effluents continued through FY 1995.Scale-up based on these processing tests will be dependent on economic assessments of the results.

\section{Office of Technical and Financial Assistance (EF)}

For this office, the Laboratory manages the Innovative Concepts Program, the States Inventors Initiative, and the International Market Assessment Program.

Innovative Concepts Program Pacific Northwest National Laboratory has managed the Innovative Concepts Program for DOE-EE since the program was established in 1983. The Innovative Concepts Program is designed to move technology more quickly from the conceptual stage to the marketplace by encouraging innovation and invention.

The Innovative Concepts Program provides "seed money" to innovators to conduct preliminary research into the technical and economic merit of concepts that have the potential to save energy, increase productivity, or reduce adverse environmental effects. An Innovative Concepts Program project cycle includes identifying a focus area, soliciting concepts, providing funding for several concepts, and introducing the concepts at a technology fair. Innovators are also given nonfinancial benefits such as the development of a one-page "tech brief" of their concept and the opportunity to attend a commercialization workshop. Over half of the 75 concepts that have been sponsored by this program have received substantial follow-on funding from industry and other government programs. 
States Inventors Initiative The Laboratory began managing the States Inventors Initiative in FY 1991. The States Inventors Initiative is designed to encourage a wider participation of inventors in helping solve the nation's energy problems. This initiative encourages and supports the formation of inventor organizations and provides information and assistance to others at state and local levels helping the commercialization process such as Small Business Development Centers. The States Inventors Initiative also facilitates the development of better methods for the inventor organizations to communicate with and provide assistance to inventors. The States Inventors Initiative has identified inventor organizations in various states. Grants have been awarded to 23 inventor organizations to support new methods to assist inventors.

\section{International Market Assessment} Program The Laboratory has conducted analysis of international energy markets for the Office of Technical and Financial Assistance for several years. The International Market Assessment Program is designed to develop information on the market characteristics, technical needs, and economic potential of international markets for U.S. efficient end-use and renewable energy technologies. Work in FY 1993-1994 focused on improving analysis efforts to understand the potential to export U.S. advanced energy technology and technical assistance in key regions, including Eastern Europe, Central and South America, and the Pacific Rim countries. Strategies for U.S. firms to operate in foreign markets through joint ventures have been studied for Mexico and will be extended to other target countries. In addition, the International Market Assessment Program provides coordination and planning support to the U.S.-supported Energy Efficiency Centers in Poland, Russia, and Czechoslovakia.

Office of Planning and Assessment (CE) Pacific Northwest National Laboratory provides technical assistance to the Office of Planning and Assessment in planning and applied analysis. In the area of planning, contributions include support in developing and implementing strategic and multiyear program planning activities, and conducting assessments of issues affecting DOE-EE programs and stakeholders.

The Laboratory is providing technical support to DOE-EE in developing the data and analytical capabilities needed to assess the potential environmental, energy saving, and economic impacts of energy efficiency and renewable technologies and programs. This includes developing a consistent total energy cycle methodology for use within DOE-EE along with supporting computer tools, participating in two interlaboratory total energy cycle analyses (bioethanol and electric vehicles), and conducting focused analyses of regulations to determine their impact on energy efficiency and renewable technologies.

As part of assessing the benefits associated with DOE-EE programs, Pacific Northwest National Laboratory is helping to develop and apply methods to estimate the environmental, productivity, economic, and equity impacts associated with DOE-EE technologies. These activities help DOE-EE understand and compare the benefits associated with competing $\mathrm{DOE}-\mathrm{EE}$ programs and technologies and also facilitate comparisons between DOE-EE technologies and more conventional choices. Finally, we are working with the Office of Planning and Assessment and other DOE-EE offices in developing an integrated information management system to facilitate access to the data, models, and other tools needed to conduct policy and program analysis.

\section{Bonneville Power Administration}

Bonneville Power Administration faces significant challenges that will dramatically alter its focus and business approach over the next 5 to 10 years. These challenges stem, to a large extent, from dramatic deregulation of the energy utility industry in general, and the electric utilities in particular. The Energy Policy Act of 1992 and recent Federal Energy Regulatory Commission decisions have deregulated the transmission and distribution of gas and electricity, opening these markets to much greater competition and encouraging many new players to enter these markets. The result will be continued pressure upon all utilities to reduce costs and increase customer service. This pressure is leading many utilities to restructure and adopt new business philosophies and priorities; this pressure is clearly affecting Bonneville Power Administration as well. In addition to industry restructuring pressures, recent drought conditions and increased demand upon regional water resources for fisheries, agriculture, and recreation further pressure Bonneville to reexamine its role and priorities.

In response to these dramatic changes, Bonneville Power Administration has embarked upon a review of a possible restructuring that would ultimately lead to becoming a government corporation similar to the U.S. Post Office and Amtrak. This decision will evolve over the next year or two and the final result is still uncertain. However, the clear message is that Bonneville will undergo dramatic change in the next 5 years.

Pacific Northwest National Laboratory's 5-year plan for supporting the needs of Bonneville Power Administration has three major elements. First, we will continue our current programmatic support in areas that are of high strategic importance to Bonneville, including energy resource programs and environmental support of fisheries issues facing the hydropower system. Second, we will continue to invest in building our capabilities to serve the new Bonneville thrust in real-time power system control. Finally, we will reevaluate our current business practices to identify new processes that better meet the needs of Bonneville, both now and in its new structure. The foundational assumption supporting this plan is that Bonneville will remain a key client and we are adapting our science and technology services to ensure that we play a vital role in Bonneville's response to its new environment.

Selected highlights of programmatic initiatives and priorities that implement these strategies are summarized below. 


\section{Real-Time Power System Control}

Bonneville operates one of the most complex and sophisticated transmission systems in North America. Maintaining high reliability while meeting load growth without addition of substantial new transmission lines will be the major challenge to be faced in the years ahead. Reliably increasing the capacity of the present transmission system will require development and incorporation of new technologies for monitoring, analysis, and control of the power system. Pacific Northwest National Laboratory is currently assisting in the development of advanced monitoring and analysis technologies for Bonneville. Development of sophisticated wide-area network monitoring will help gather much needed, time synchronized data enabling development of improved system models and examination of dynamic behavior not presently well understood. Additionally, a new suite of analytical tools for examination of this data is being developed with Bonneville and researchers at the University of Colorado. Finally, a special controller is being developed for application at the EPRI-GE flagship device for power system control, the Thyristor Controlled Series Capacitance unit now undergoing acceptance testing at the Slatt Substation in northcentral Oregon.

It is anticipated that the technology development and deployment by the Laboratory in conjunction with Bonneville will continue for at least the next 5 years. It is reasonable that widearea network monitoring systems will be deployed across the entire western U.S. This will be followed by analysis of monitored behavior, development of improved analysis tools, and utilization of these tools to improve performance of existing power system stabilizers, progressively including improved control of the major elements of the transmission system such as the HVDC transmission line to California. Additionally, improved monitoring will be incorporated in high-level, on-line and real-time analysis and control of the power system using many of the technologies currently under development, eventually including artificial intelligence systems. This program has been selected for the EPRI-DOE SEP alliance, leading to greater industry involvement.

\section{Fish and Wildlife Programs}

The Bonneville Power Administration is charged by Congress to protect and enhance the fish and wildlife of the Columbia Basin while providing power to the region. The Laboratory is an important scientific and technical resource to Bonneville for planning and implementing the Fish and Wildlife Program.

Today, many of the natural resources in the Pacific Northwest continue to decline. The Laboratory is supporting Bonneville's efforts in many areas to reverse these trends, including fisheries habitat restoration and mitigation planning. To support these emerging Bonneville needs, we are targeting research in integrated environmental monitoring; computer based controls for natural resource monitoring facilities; image acquisition, processing, and data management; fisheries physiology; experimental design and hypothesis testing; facilities certification; fisheries genetics; and evaluation of electromagnetic exposures.

\section{Regional Energy Resource Programs}

Pacific Northwest National Laboratory has supported a broad range of Bonneville Power Administration resource programs over the past decade that include strategic support of energy efficiency and renewable generation research and development, development of resource planning data and methods, and efficiency program design and evaluation. Current and future efforts will focus primarily upon industrial and manufactured housing needs.

The overall goal of the Industrial Demand-Side Management Project is to determine, confirm, and develop the conservation resources that can be achieved from operations and maintenance practices in Pacific Northwest industries. The project is currently establishing methodologies for process auditing, data acquisition, and data analysis. This will support evaluation of the operations and maintenance practices for electric energy resource potential of industrial air compressors and compressed air systems. Future activities will include industrial plant audits and baseline data acquisition, implementing audit recommendations, monitoring and analyzing results, and technology transfer.

A future priority for Bonneville's industrial impact evaluations is estimating how much energy is saved over the long term (persistence of savings). The Laboratory will be developing a methodology for evaluating this aspect of demand-side management performance.

About one-third of the new homes in the Pacific Northwest are HUD-code manufactured homes, built to a relatively loose national thermal code. Through technology and information transfer, the Laboratory is assisting Bonneville in sharing information on its energy-efficient manufactured homes program with the states, manufacturers, and other interested parties. In 1992, Bonneville initiated the Manufactured Housing Acquisition Program (MAP), a market transformation program directed at the home manufacturers. By paying the manufacturers $\$ 2,500$ for each energy-efficient, electrically heated MAP home, Bonneville has succeeded in encouraging the manufacturers to build homes that use approximately half the energy required to heat a typical manufactured home. The saved energy is being acquired at a cost which is about half that of building a new power plant. More than 25,000 homes have been built to MAP standards.

Because a new, tighter national code went into effect in October 1994, Bonneville and manufacturers are looking at ways to modify the MAP. The Laboratory will be assisting Bonneville in identifying the economic effects of the new code on the assumptions underlying the current MAP and helping develop creative ways to modify the program. The Laboratory also has initiated work on a program evaluation of the impacts of MAP by collecting utility billing data and additional information from owners of 
manufactured homes. Information from this evaluation will be useful in assisting Bonneville and utilities in developing future conservation programs that aim to transform the market, rather than just affect selected situations.

\section{Office of Civilian Radioactive Waste Management}

Pacific Northwest National Laboratory programs supporting the Office of Civilian Radioactive Waste Management (RW) cover several activities. Yucca Mountain Site suitability studies continued as did limited onsite activities. Activities were completed to transfer the Laboratory's scope to the DOE-RW management and operations contractor.

\section{Program Management and Integration (DB)}

The Systems Integration Program supports the DOE-RW Office of Program Management and Integration by ensuring that components of the nuclear waste management system are integrated into an efficient, safe, and timely waste management system. Components may include a mined geologic repository, waste generators, nearterm storage, transportation, and a monitored retrievable storage facility. The Systems Integration Program technology and program scope were successfully transferred to the management and operations contractor.

The Laboratory role is currently directed toward providing the Office of Program Management and Integration with limited independent technical review and consultative support.

\section{Waste Acceptance, Storage, and Transportation (DB, DC)}

Pacific Northwest National Laboratory has provided planning and technical management to $\mathrm{DOE}$ for the national Commercial Spent Fuel Management Program. Although the program came to an end, efforts begun by the Laboratory under the Commercial Spent Fuel Management Program are continuing as independent projects. Focus of continuing research and development being done with the Laboratory's involvement is on establishing technology that nuclear power utilities can use for interim spent fuel storage efforts.

The Laboratory provides technical management for cooperative programs between DOE and utilities, including participation of the Electric Power Research Institute. Included in cooperative program activities is the establishment of an agreement to design and demonstrate a licensed system for the dry transfer of spent nuclear fuel between two licensed store-now/ transport-later dry storage systems.

Under the Heat Transfer Code Evaluation/Qualification Project, the Laboratory is responsible for evaluation, qualification, maintenance, and user support for COBRA-SFS and HYDRA-II which are "best estimate" thermal-hydraulic computer codes for spent fuel dry storage systems. The codes have been extensively validated and have undergone technical evaluation by the Nuclear Regulatory Commission for use in licensing analyses of spent fuel storage systems.

The Pacific Northwest National Laboratory Utility Interface Programs assist the DOE-RW's Office of Waste Acceptance, Storage, and Transportation and the DOE-RW management and operations contractor in performing the waste acceptance function. We support preparation of two annual DOE reports to implement the DOE contract with utilities to accept their fuel. The Acceptance Priority Ranking represents the DOE contractual commitment to accept spent nuclear fuel for disposal. The Annual Capacity Report, required by the contract, sets forth receiving capacity for the waste management system and allocates the appropriate share of the capacity to the contract holders. The Laboratory assists the Office of Waste Acceptance, Storage, and Transportation with the development and implementation of the control systems for managing delivery commitment schedules.

In addition, the Laboratory helps in reviewing, reconciling, and disseminating the data on spent fuel inventory and projections that are collected for the utilities. These data form the bases for planning the waste management system.

\section{Policy Office}

Pacific Northwest National Laboratory provides assistance to the Policy Office (PO) in the area of economic and policy analysis, both on major long-range studies and on short-turnaround issues as they emerge. The main strength of the assistance the Laboratory provides to the Policy Office is its policy relevance. As an example, we embarked on an ambitious project to increase international understanding of the challenge to and understanding of the role of social sciences as global climate change is addressed. Under the working title, Human Choice and Climate Change: A State-of-the-Art Report, the SOAR project has been working with an international team drawn from a wide range of the social sciences. The interdisciplinary team will analyze current social science contributions to climate change information to determine what is known, unknown, and uncertain; to evaluate the strengths and weaknesses of social science knowledge; and to identify new areas of potentially fruitful research.

The Laboratory's extensive modeling capability provides another valuable input to the policy decision process. The Edmonds-Reilly Long-term Global Energy- $\mathrm{CO}_{2}$ Model, which we maintain and update, continues to be used extensively in scenario analysis for numerous studies of possible greenhouse gas control policies. In addition, significant improvements in the forecasting of greenhouse gas emissions are expected progressively over the next few years as our efforts to develop the Second Generation Model come to fruition. Currently, we have completed six modules, including the U.S., Western Europe, Korea, Japan, Canada, and India. Modules for China and Mexico are near completion. Modules for the Former Soviet Union, Australia, Brazil, Other Latin America, and Africa are in the planning stages. The Second Generation Model program is founded on the principle that 
a fully international program can achieve more than even the best national program. Each nation (region) is unique in its institutional arrangements, data collection, aggregation methods, and political setting. The Second Generation Model has been designed to allow the inclusion of unique regional characteristics while requiring common module functions and data definitions.

The Laboratory continues to assist the U.S. government in the efforts of the Intergovernmental Panel on Climate Change (IPCC) in developing the Second Assessment. Specifically, the Policy Office is providing funding to lead authors and contributing authors on Working Groups 2 and 3 in the areas of Energy Supply and Human Settlements. Second order drafts were developed in FY 1995 for international government review. The authors attended several international meetings as part of that process. The Second Assessment is scheduled to be published in December 1995.

Pacific Northwest National Laboratory is assisting the Policy Office with several projects related to requirements established by the Energy Policy Act of 1992 (EPAct). Reports were completed for sections 1601 and 1605 during FY 1994. EPAct Section 1604 requires DOE to assess alternative policy mechanisms for addressing greenhouse gas emissions. For the EPAct 1604 requirement, the Laboratory is developing a draft Report to Congress, containing a micro-level analysis of the potential implications and drawbacks of two categories of policy instruments that may be applied to reduce greenhouse gas emissions: economic instruments and energyefficiency standards.

For the future, the Laboratory plans to continue to provide the policy-relevant analyses required by the Policy Office. Future issues for the office will most certainly be global issues, as global climate change issues will likely remain in the forefront for the long term. Such issues as large-scale regional issues, sustainable development, and global environmental equity are also likely to remain important issues for the foreseeable future. The Laboratory expects to support the Policy Office in contributing to U.S. actions conducted as part of the U.S. Joint Implementation Plan. Joint Implementation efforts are undertaken by more than an individual country to reduce climate change emissions.

Pacific Northwest National Laboratory also provides assistance to the Policy Office in examining the impact of energy and economic policy on the efficiency of energy use in major end-use sectors. For example, there are three efforts under way for the Policy Office's Economic Policy Office. The first is an input-output study that includes an analysis of the respective roles of industrial energy-efficiency improvements and changes in the composition of final demand in changes in economic productivity. Building on past cooperative DOE-PO/EE work, it will enable the Policy Office to complete an analysis of Japanese-American industrial energy use comparisons as well as similar comparisons with Canada. This work will also be useful in the context of global climate change issues. The second is an update of an analytical tool called SEADS-PC, the Sectoral Employment/Energy Analysis Data System. This tool also uses input-output analysis to translate policy changes interpreted as changes in final demands into changes in energy use and/or employment. This work is nearing completion and has been used to analyze the impact on employment of the President's Climate Change Action Plan. The third effort will provide rule-of-thumb estimates for the employment effects of an energyefficiency investment undertaken by DOE. The analysis will reflect a weighted average of the direct and indirect employment effects as a consequence of alternative types of investments.

Additional work is under way for the Policy Office's Office of Energy Efficiency and Alternative Fuels to investigate strategies for reconciling compliance with environmental requirements with improvements in productivity and energy efficiency, focusing specifically on the paper and steel industries. Other projects address how computer models can best be used to evaluate potential energy policies.

\section{Office of Nuclear Energy}

Pacific Northwest National Laboratory increased its participation in the DOEOffice of Nuclear Energy International Nuclear Safety Program in FY 1995. Current and projected funding for the Office of Nuclear Energy (NE) programs are shown in the Resource Projections.

Over the planning period, the Laboratory plans to increase its participation in DOE-NE research and development programs in the areas of the storage, use, and disposal of surplus plutonium from arms reduction; actinide burning; radioactive waste minimization; isotope production and distribution; and advanced reactor design review and certification.

Nuclear energy programs to meet future power needs in the U.S. are not expected to expand until the latter part of the planning period. The Laboratory plans to participate in activities aimed at revitalizing nuclear power as these programs develop.

\section{Nuclear Energy Research and Development (AF)}

The Laboratory supports the Office of Nuclear Energy in its efforts to assist the Former Soviet Union in nuclear reactor safety. DOE-NE is the primary provider of technical assistance within the U.S. government. The nuclear reactor systems designed and constructed by the Soviet Union, particularly RBMK and VVER reactors, do not meet Western standards for nuclear safety. At the same time the Former Soviet Union states have concluded that these reactors are required to provide energy to support their struggling economies. The Laboratory has been designated as the primary DOE laboratory responsible for providing project management and technical and administrative support for the International Nuclear Safety Program. FY 1996 funds to be directed by the Laboratory are expected to be $\$ 57$ million with continuing efforts at about this level 
throughout the planning period. The objective of the program is to reduce the risks of Soviet-designed reactors by working cooperatively with host countries to strengthen their nuclear safety cultures and supporting infrastructures. Elements of the program include Safety Regulation and Legislative Requirements, Plant Safety Evaluation, Management and Operational Safety, Engineering and Technology Safety, Fuel Cycle Safety, and Program Management and Integration. The program provides assistance to Russia, Ukraine, and other Central and Eastern European countries. U.S. participants also include other DOE laboratories and U.S. industrial firms.

\section{Radioisotope Production and Use}

Pacific Northwest National Laboratory plans to conduct research and development in support of Hanford's role as the DOE's production and sales site for cesium-137, strontium-90, and other selected isotopes. The Laboratory is developing separation processes for obtaining various isotopes for medical applications. In addition, work is under way to develop new compounds containing radioisotopes for specific treatment of selected organs or other body parts.

\section{Office of Fossil Energy}

Pacific Northwest National Laboratory's involvement in the DOE's Fossil Energy programs is expected to continue to increase because of the current DOE investments in research related to the natural gas and oil industry, environmental regulation, energy conversion, gas and coal chemistry, advanced materials development, and environmental effects of coal use.

Regulatory compliance has become the central area of research that can assist the competitiveness of the industry. The $\$ 37$ billion estimated to be required between 1991 and 2000 for plant upgrades to produce reformulated fuels required under the Clean Air Act Amendments is only $\$ 6$ billion less than the current book value of all U.S. refineries. The U.S. refining industry faces environmental compliance costs totaling at least $\$ 150$ billion through 2010. The purpose of the Refinery of the Future Initiative and the national Gas and Oil Technology Partnership is to develop research solutions to problems that DOE and the petroleum industry have in common. These partnerships are designed with industry participation and collaboration and incorporate a wide variety of cooperative mechanisms.

The Natural Gas and Oil Technology Partnership was formed 5 years ago by the three Defense Program Laboratories (Sandia National Laboratories, Lawrence Livermore National Laboratory, and Los Alamos National Laboratory). With the implementation of the Advanced Computational Initiative in FY 1995, the partnership was expanded to include the Energy Research laboratories. The Advanced Computational Initiative program focuses on exploration and production computing technology. It is funded at $\$ 42$ million for FY 1995 through the offices of Fossil Energy, Energy Research, and Defense Programs. Pacific Northwest National Laboratory is committed to actively participating in the Advanced Computational Initiative. We have technical capabilities in molecular computation and design, data fusion, multiphase fluid flow, and several other areas that could be applied to achieve the goals of the Initiative.

DOE has recently announced the Domestic Natural Gas and Oil Initiative to stimulate job creation, increase production, and reduce reliance on foreign oil. The Laboratory plans to work with DOE-FE to understand how the environmental remediation and waste management experience (such as risk assessment, contaminant transport, environmental characterization, and monitoring) can be brought to bear on the environmental issues that these industries face. In a related initiative, DOE-FE and DOE-EE are focusing on both technical processing and environmental issues to ensure a continuation of a robust, highly competitive refinery of the future.

The Advanced Materials and Electrochemical Processes program which includes solid-oxide fuel cells, membranes, and catalytic processes for energy and environmental applications, continues to grow with a major effort being made to expand this program within DOE-FE and extend the program to better support industry. The program focuses on high-temperature materials related to electrochemical, catalytic, and membrane technologies for broad, clean, and efficient use of coal and other fossil fuels. The program emphasizes materials and processes related to catalysts and inorganic membranes for energy conversion, minimization of waste contamination, and synthesis of materials. These developing concepts can also be directly combined with other technologies to further enhance the beneficial use of fossil and other fuels.

Another program, Advanced Materials for Solid-Oxide Fuel Cells, emphasizes the major generic issues of solid-oxide fuel cells performance and manufacturing. The research and development emphasizes critical issues of materials processing, fabrication, and performance. It is anticipated that the Laboratory will expand its involvement with U.S. industry to provide development support for more efficient manufacturing of advanced solid-oxide fuel cells in areas of CRADAs and technology transfer. It is anticipated that the Laboratory will expand research and development on solid-oxide fuel cells through involvement with U.S. companies in developing the fuel cells. Programs also are being directed to provide support for more efficient manufacturing of solid-oxide fuel cells.

This research is associated with the Laboratory's molecular sciences research activities and utilizes interdisciplinary research to gain an understanding of the processes related to catalytic, high-temperature membrane, inorganic, and electrochemical technologies.

The Laboratory's research and development for solid-oxide fuel cells continued in FY 1995 and will continue beyond. These efforts will continue to expand and include CRADAs and private support with industry in areas of material synthesis, processing, 
fabrication, and performance for application to solid-oxide fuel cells.

Methods employing nuclear magnetic resonance and gel permeation chromatography are being developed for determining the structural analysis and molecular weight of coals that have been degraded to forms of lower molecular weight by microbial treatment. Although analysis of biodegraded coals will remain an important research function at the Laboratory, projects are planned that will increase our involvement in coal liquefaction technology development. The Laboratory has begun research to synthesize ultrafine catalyst particles for coal liquefaction. Ultrafine catalyst particles less than 10 nanometers in diameter have high surface areas and the ability to penetrate into coal pores. These properties can decrease the quantity of catalyst used for liquefaction and increase liquefaction rates and selectivity. The catalyst particles are prepared using novel refinements in the emerging technology of reverse micelle inorganic chemistry and for thermal expansion/decomposition methods.

A 3-year CRADA has been established between Pacific Northwest National Laboratory and Phillips Petroleum Company to develop a novel Laboratory process for treating spent catalysts from petroleum refineries. This process uses electrochemistry to remove contaminants that accumulate during processing that result in decreased efficiency. The technology is more environmentally friendly than conventional methods of dealing with spent catalyst and provides opportunities to improve conversion of crude oil to transportation fuels.

\section{National Security}

Pacific Northwest National Laboratory's national security activities are derived from and support the DOE's national security core business area. The historical role of the Laboratory and its predecessor laboratory in defense materials production, the early tasking by the Atomic Energy Commission to develop sensitive radionuclide collection and analysis capabilities to monitor worldwide radioactive fallout and Hanford Site sources, and its continuing support to each of the DOE's mission areas are the basis for the Laboratory's current national security mission.

The new world order has resulted in rapidly changing national security policies, needs, and challenges, marked by regional, ethnic, and national conflicts; increased concern regarding proliferation of nuclear weapons and other weapons of mass destruction; treaty verification and arms control requirements; and maintenance of a technical base in support of DOE's national role in nuclear materials management and nuclear weapons stewardship.

Our national security objective is to integrate the full spectrum of technical knowledge at the Laboratory to anticipate and rapidly respond with creative and innovative solutions that enhance national and international security. Our goal is to provide an objective and independent national technical resource to national and international agencies and industries to

- support policy development and implementation planning

- develop and apply technology to implement national security policy

- provide technologies to meet national security requirements generated by the disparate threats across the broad spectrum of mission requirements.

The Laboratory has a growing, technologically rich, and well-recognized role in six focus areas: arms control and nonproliferation, intelligence, safeguards and security, defense systems technologies, defense production and logistics, and technology research.

The Laboratory's national security work is principally conducted for DOE's Office of Nonproliferation and National Security, Office of Defense Programs, and other federal agencies through the Work for Others Program.

Our national security work is conducted throughout the Laboratory, thereby ensuring that the full resources of the Laboratory are applied to meet programmatic needs. There are real and recognized interfaces and overlaps with the other areas of the Laboratory. These interfaces are strengths in meeting national and international challenges not only in national security but the other business areas as well.

Two Laboratory Directed Research and Development (LDRD) initiatives started in FY 1995: the Intelligence Information Technologies Initiative and the Rapid Prototyping Initiative. The Laboratory-level Medical Systems and Technologies LDRD Initiative also has its origin in National Security.

Intelligence Information Technologies Initiative (IITI) The Intelligence Information Technologies Initiative is aimed at developing the Laboratory's resource base in information management to a major capability level that is nationally recognized. Our strategic approach is to focus on the specific needs of the intelligence community where information technologies and systems to generate tools and create infrastructure changes will result in the optimum distribution of analysts' time and improvements in production efficiency. This demand has been generated because of

- shrinking budgets, hence fewer analysts

- increase in analytic tasking

- greater number and diversity of targets for analysts

- rapidly growing volumes of available information

- diverse types and sources of information (classified and open source).

The Laboratory is pursuing technical research in specific areas of information technologies. The approach is to build on existing capabilities and project experiences along with current activities that link the Laboratory directly to the DOE and other members of the intelligence community.

The research emphasis in this initiative is to develop enabling technologies that will support a variety of applications. Three technical thrusts have been identified: 1) provide seamless access to heterogeneous databases and develop active agent browsing, 2) provide a 
mathematical baseline for natural language processing and define text browsing in an analytic framework, and 3) develop an analyst's workstation environment with optimal incorporation of intuitive user interface factors. As these technologies are developed, it is expected that they and related projects will be infused and stimulated throughout the Laboratory. The goal is to increase our existing baseline of information technologies and to propose additional applications to external users/clients.

Rapid Prototyping Initiative Solid freeform fabrication is a revolutionary manufacturing method used to rapidly build three-dimensional objects from computer-aided design solid models. This "rapid prototyping" technology reduces product development cycle times, as it requires virtually no machine setup. Cost for one solid freeform fabrication object is roughly the same as for 1000. It is an ideal candidate for production of prototypes and tools. Applications such as rapid fabrication of stamping, forming, and molding dies; rapid development and market testing of new products; and microfabrication of three-dimensional parts each have the potential to transform industry and society.

This work is intended to facilitate Laboratory efforts in solid freeform fabrication research and development being conducted to meet national security and industry needs for rapid, low cost, highly flexible manufacturing technologies. If the materials usable for the solid freeform fabrication technique are extendable to metals, ceramics, and other materials capable of being used as forming, molding, or casting tooling, solid freeform fabrication could become a national competitive advantage for the next century.

Pacific Northwest National Laboratory's emerging initiative involves developing industrial relationships with relevant partners, and two initial research efforts. One is a project in ceramics funded by 1995 LDRD, and the other is a continuation of 1994 work in microfabrication, with collaborations with Penn State University and Oregon State University.
Outcomes intended from such coordinated efforts include 1) partnerships with current rapid prototyping vendors to infuse technology for immediate process improvement; 2) partnerships with industry and other government agencies to develop new processes capable of meeting specific application requirements; and 3) a DOE user facility in support of small manufacturing businesses in the Northwest. Several key industry and government contacts have also been made. These include General Motors, Ford, Coors Ceramic, Lockheed, Hewlett Packard, Westinghouse, DOE, ARPA, Penn State, University of Michigan, SIRTI, Los Alamos National Laboratory, Sandia National Laboratories, Oak Ridge National Laboratory, National Aeronautics and Space Administration Lewis, and solid freeform fabrication technology developers/vendors such as DuPont, Helisys, and Incre.

\section{Office of Defense Programs}

Pacific Northwest National Laboratory's work for the Assistant Secretary for Defense Programs includes safeguards and security activities in direct support of defense programs, participation in the production of special isotopes, and technical support to the nuclear weapons complex.

The Laboratory also assists Defense Programs in interpreting safeguards and security policies and identifying costeffective implementation procedures for the Defense Programs complex.

The production of special isotopes (Materials Support [GE]) often requires the capabilities of several sites and contractors because of size, space, or other limitations. The Laboratory has unique capabilities for handling long reactor fuel and reactor components. We continue to support the Mark-42 target program. The Mark-42 targets are used for the production of plutonium-242, americium-243, and curium-244.

We also continue to provide technical support to the Defense Programs complex in specific materials support activities. The Laboratory is working closely with the Office of Military Application and Stockpile Support (DP-20) to define a light water reactor tritium target qualification programa program designed to qualify the target system design first demonstrated in a previous Tritium Target Development Program initiated under the DOE New Production Reactor Office between FY 1989 and FY 1992. The qualification program would provide a tritium target system that could be used with confidence in the DOE's contingency option for tritium production for nuclear weapons applications.

\section{Office of Nonproliferation and National Security}

Pacific Northwest National Laboratory support to the Office of Nonproliferation and National Security is provided to the Office of Research and Development, Office of Energy Intelligence, Office of Arms Control and Nonproliferation, the Office of Security Affairs, and Office of Emergency Management. The Laboratory participates in the Executive Council of the Office of Nonproliferation and National Security and provides direct support as requested.

The Office of Nonproliferation and National Security provided support in FY 1993 for the Laboratory to prepare a Conceptual Design Report and Validated Cost Estimate for the proposed construction of a new DOE facility at the Hanford Site dedicated to support of the Office of Nonproliferation and National Security and other classified work. This continued to be a validated proposal in FY 1994 but budgetary impacts resulted in this proposed facility being dropped from consideration in FY 1995.

\section{Office of Research and Development}

Pacific Northwest National Laboratory's capabilities are applied to research, development, and application of state-of-the-art sensors and sensor systems and the statistical evaluation of their responses. Emphasis has been placed on nuclear radiation technologies and nondestructive testing, which 


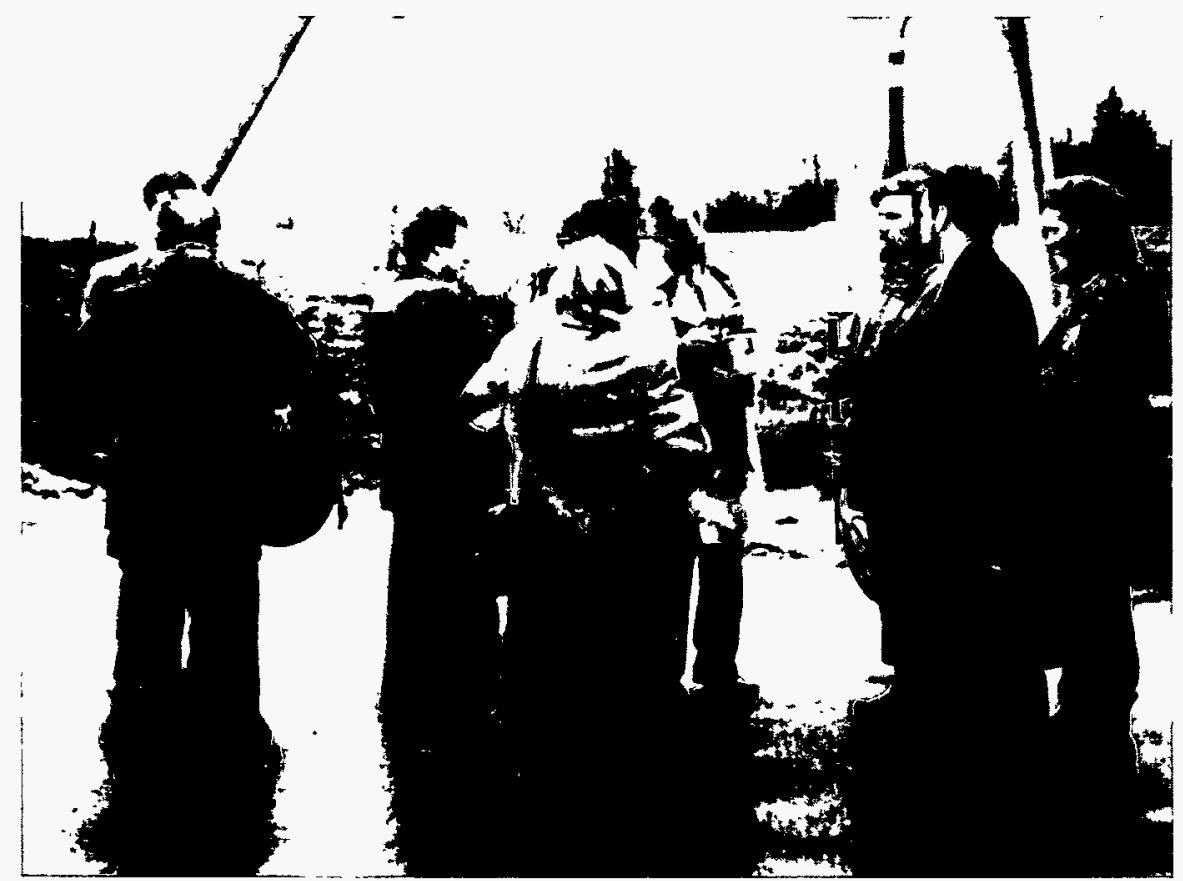

Laboratory staff member examining a portable radiation monitor used by the Lithuanian border guards at the Lithuanian/Belarus border where 2 tons of radioactive tungsten had been seized when there was an attempt to smuggle it across the border. The Laboratory, working with the U.S. Customs Service, performs assessments of enforcement capabilities in Former Soviet Union states and then provides training to these countries. We provide training on nonproliferation of weapons of mass destruction.

are expected to have a significant role in formulating protocols and for monitoring existing arms-control treaties. Remote sensing science and digital image processing have been developed for applications supporting treaty requirements.

A critical area of concern is proliferation of nuclear, chemical, and biological weapons and their associated delivery vehicles. The DOE has an objective to detect and assess nuclear proliferation and to ensure that the U.S. government has the ability to enter into and evaluate compliance with existing and future bilateral and multilateral agreements controlling nuclear weapons and their proliferation. The Laboratory is focusing its national security technology capabilities to identify needs and develop technologies to monitor, detect, and analyze for indications of such proliferation.

We support the DOE's treaty involvement through technology development, expert knowledge, and onsite support in Washington, D.C.; the Conference on
Disarmament in Geneva, Switzerland; the United Nations Special Commission (UNSCOM); and the International Atomic Energy Agency (IAEA).

\section{Office of Energy Intelligence}

Pacific Northwest National Laboratory directly supports the DOE Office of Energy Intelligence in areas of nuclear nonproliferation, energy assessment, and intelligence support. We perform intelligence data processing and analyses that address national issues in weapons materials production, nonproliferation, and energy resources, and other tasks as appropriate. The Laboratory further supports studies and application of technologies in special programs. Products and services provided to the DOE Office of Energy Intelligence contribute to the support of policymakers and the intelligence community. Additionally, in coordination with DOE's Office of Energy Intelligence, the Laboratory applies its capabilities and technologies to perform intelligence-related work for other government organizations.

\section{Office of Arms Control and Nonproliferation}

Pacific Northwest National Laboratory directly supports the DOE's Office of Arms Control and Nonproliferation in the specific functional areas of policy and technical analysis, export control, international safeguards, and international and regional security. The Laboratory activities are expected to increase during the planning period in support of national policies and interests in nonproliferation, treaty requirements, nuclear disarmament, and nuclear threat reduction. The Laboratory projects supporting the objectives of DOE's Office of Arms Control and Nonproliferation draw upon the Laboratory's substantial expertise in nuclear materials production and other national security activities.

The Laboratory is a primary resource for the DOE Office of Arms Control's Negotiation and Analysis Division, providing scientific, engineering, and operations expertise from the DOE weapons material production complex for the negotiation and implementation of arms control treaties and agreements. In support of Fissile Material Cutoff Treaty negotiations, we have facilitated the use of the Hanford Site, a past weapons material production site, to provide "real world" familiarization and insight for the ambassadors negotiating the treaty. The Laboratory staff support DOE in the preparation, review, and analysis of U.S. government policy positions/options for the cessation of Russian plutonium production. A Laboratory staff member is also assigned as DOE's representative on the U.S. delegation negotiating modifications to the Anti-Ballistic Missile Treaty. These examples illustrate the breadth of our arms control policy involvement, extending from the beginnings of policy formulation, through treaty negotiation, to follow-on treaty implementation.

The Laboratory's capabilities are applied in support of the DOE's role in developing the nuclear-related technology sections of the Military Critical Technologies List. The Laboratory supports DOE efforts with the 28-country Nuclear Suppliers Group that develops and institutes multilateral export 
controls to non-Nuclear Suppliers Group countries. The Laboratory staff assisted the Nuclear Suppliers Group by providing the technical justification for changes and modifications to the former IAEA list and the U.S.'s recommendations for nuclear dual-use export control. In addition, we provide other classified services to DOE in the technology export arena and chair a multiagency technical review group that addresses sensitive nuclear issues. The Laboratory acts as a technical reviewer for DOE on specific authorization requests by U.S. companies who wish to assist foreign atomic energy activities. The Laboratory also is a member of a U.S. delegation headed by the U.S. Customs Service to assess and train Former Soviet Union nonweapons states on export controls of strategic and dual-use materials.

Pacific Northwest National Laboratory participates as a member of the Materials Protection, Control and Accounting Steering Committee coordinating the DOE "Lab-to-Lab" efforts to improve safeguards on nuclear weapons materials in the Former Soviet Union, particularly the Russian Federation. Laboratory staff are involved in many of the tasks associated with this highly visible and growing U.S. government activity. We also support the Office of Arms Control and Nonproliferation in the effort to place excess weapons plutonium at the Hanford Site under international safeguards, according to the President's Voluntary Offer.

The Laboratory is a participant in the Industrial Partnering Program with the New Independent States (NIS/IPP) of the Former Soviet Union. The goals of this joint Department of StateDepartment of Energy program are to 1) stabilize supporting technology equipment and facilities to enhance global nonproliferation; 2) develop advanced technology commercial opportunities and markets; 3 ) enhance U.S. science and engineering capabilities; and 4) engage weapons scientists, engineers, and technicians in nonweapon activities. This program is cooperatively managed by a 10 -member Interlaboratory Board that is responsible for establishing guidelines for projects. Projects supported by NIS/IPP are classified in two thrusts. Thrust I projects are cooperative research projects between U.S. national laboratories and NIS institutes. Thrust II projects are intended to provide market opportunities for technology jointly developed and/or manufactured and are carried out in a three-way partnership that includes a U.S. industry representative. The U.S. industry representative is required to be a member of the United States Industry Coalition, and is expected to provide matching funds in order to participate in whatever market opportunities arise from the collaborative work.

Laboratory staff have recently been part of the DOE/U.S. government effort to ensure safeguards control over North Korean spent nuclear fuel. Laboratory staff were part of the first U.S. group to visit the North Korean nuclear complex at Yongbyon in direct support of this activity.

\section{Office of Security Affairs}

Pacific Northwest National Laboratory activities for the Office of Security Affairs cover five major areas that provide strong linkages across the Nuclear Safeguards and Security Program. Each of the major activities addresses significant portions of the overall protection program and focuses on integrating these portions to enhance the effectiveness and efficiency of the operation. The primary areas addressed by the Laboratory include 1) Information Security planning, policy, practices, and procedures; 2) Material Control and Accountability technology transfer, performance-based policy implementation, technology and procedural alternatives; 3 ) management assessment techniques and resource review support; 4) national level initiatives of Arms Control and Nonproliferation including technology development and deployment; and 5) Counterintelligence Assessment and Training Program activities.

Major assumptions used in the formulation of these activities are 1) DOE's assets requiring protection have increased but changing missions require consolidation and more efficient use of technologies to reduce operational costs and facilitate accessibility to the complex, 2) national level policy on security is undergoing dramatic changes, 3) significant changes are required at DOE facilities to prepare for the implementation of arms control and nonproliferation treaties that will permit intrusive inspections by international agencies and foreign individuals, 4) DOE's openness initiatives and economic competitiveness must be addressed, and sensitive technology and some level of the nuclear weapons program must be protected in this environment, and 5) DOE Headquarters organizations and field components must work in concert to solve issues that cross traditional management structures (i.e., safety, health physics, safeguards, etc.).

The development of performancebased implementation guidance that recognizes the need for flexibility across the DOE complex and the ability to provide assistance to field elements to implement this guidance will strengthen DOE-Headquarters' support to field elements and facilitate the implementation of cost saving safeguards and security measures while meeting customer and stakeholder needs.

The preparation of DOE facilities for inspections under the auspices of international treaties will provide visibility for DOE as a leader in the formulation of national security-related policies and procedures and will further the success of the associated national level initiatives.

The identification, dissemination, and transfer of technology, practices, and procedures across the DOE complex will ensure that a state-of-the-art safeguards and security program is in place and capable of providing effective and efficient protection for Department assets, including accountability and control of special nuclear material.

This program is directed toward supporting DOE's overall mission, and the success of these activities will lead to the Department's ability to open many facilities for decontamination, decommissioning, and alternative uses.

Laboratory support to Security Affairs is expected to remain stable but the 
technical challenges should grow in complexity as the operational goals of DOE continue to change.

\section{Office of Emergency Management}

Pacific Northwest National Laboratory supports the Office of Emergency Management in the determination of technical requirements and the development and deployment of technical systems in support of mission needs. The Laboratory has worked cooperatively with Lawrence Livermore National Laboratory, Oak Ridge National Laboratory, and Los Alamos National Laboratory to provide DOE with a plan that addresses the worldwide problem of nuclear smuggling. The Laboratory also participates in the coordination with other government agencies led by this office.

\section{Economic Productivity}

Industrial partnerships play an important role in enhancing Pacific Northwest National Laboratory's core competencies and in meeting the goals of the Department of Energy's four core missions. As a multiprogram laboratory, Pacific Northwest National Laboratory believes that working cooperatively with industry enhances research results and leverages both private and public funding, while having the benefit of promoting economic growth and the creation of high-wage jobs. As an organization managed and operated by Battelle whose fundamental mission is "Putting Technology to Work," the Laboratory has a unique base of technical skills and business experience that it can apply to U.S. industry's needs.

For over 25 years, the Laboratory has been managed and operated under a unique partnership arrangement between DOE and Battelle that allows Battelle-owned facilities to be used in the conduct of federally sponsored activities and DOE-owned facilities to be used in the conduct of activities sponsored by private industry. The contractual arrangement governing this partnership is called the Use
Permit, which allows Battelle to contract directly with private industry for the conduct of research and development activities by Laboratory staff.

The Use Permit has proved to be a valuable mechanism for facilitating the use of the Laboratory's technical resources to assist U.S. industry and for tailoring available technology for application by industry. At the beginning of FY 1995, our active contracts with industry under the Use Permit totaled 218 and had a value of $\$ 72$ million. The Laboratory also has an extensive track record in encouraging the development of new "spin-off" companies. Over 40 companies are currently in existence whose technical roots can be traced to the Laboratory.

The Laboratory's long history of working with industry provides us with a unique perspective on how to leverage federal investments in mission oriented research to solve industry problems.

In this context, the Laboratory's objectives for improving U.S. economic products are to

- develop and implement technology partnerships with industry segments, other national laboratories, and academia

- balance market pull with technology push in Laboratory programmatic activities

- shorten the technology life cycle from research to commercial deployment through cross-functional parallel activities

- assist government and industry in moving from cleanup to waste minimization and pollution prevention (i.e., toward "sustainability")

- leverage federal investments and the combined talents of the national laboratory system and Battelle to diversify the local Hanford and Northwest regional economies, strengthen their export potential, and create new high-wage jobs.

Pacific Northwest National Laboratory's strategy for achieving these objectives is to increase industrial awareness, provide technical assistance to industry, collaborate with industry through partnerships (including CRADAs), and commercialize intellectual property. We believe technology transfer is an integral part of everyone's job and are continuously developing new initiatives as a means of enhancing our performance.

\section{Enhanced Awareness of Industrial Needs Throughout the Laboratory}

Staff awareness of industrial needs is achieved through various means. Technology transfer liaisons, for example, are assigned within the Laboratory research divisions, and a CRADA facilitator is designated for each Program Secretary Office. These liaisons continuously inform and advise staff about technology transfer mechanisms, incentives, and important opportunities to perform collaborative research with U.S. industry.

The Laboratory supports the new DOE Integrated Technology Transfer System (ITTS) through participation with the ITTS Working Group. Points of contact for the seven technology transfer mechanisms have been selected for leadership and coordination of data gathering. Information has been input for several areas including CRADAs and licensing. This activity will continue at the Laboratory during FY 1996 as the Laboratory team proceeds with populating the database.

The Laboratory has an extensive Recognition and Rewards program that includes a royalty-sharing program (modeled after the "best-of-the-best" from similar federal and industrial programs). An annual "Technology Transfer Day" at the Laboratory also recognizes contributors to the technology transfer process (e.g., those receiving patents and copyrights or the R\&D 100 and FLC award winners during the past year).

In the past year, we have also completed the following specific actions

- increased the number of presentations on technology transfer throughout the Laboratory 
- proposed a new entrepreneurial leave of absence program to improve technology transfer locally through increased formation of spinoff businesses by Laboratory staff

- offered a half-day course on conducting market assessments and twice offered a 2-day course on Industrial Marketing for STEs; these were each attended by over 30 staff members.

\section{Enhanced Industrial Awareness of the Laboratory}

To accomplish this objective, Pacific Northwest National Laboratory produces and uses a broad range of communication mechanisms, from visits, telephone calls, tours of Laboratory research facilities, workshops, and other important one-on-one contacts to press releases, brochures, and exhibits at trade shows attended by thousands. These outreach techniques and other activities are designed to increase interactions significantly between Laboratory staff members and their industrial counterparts. We also take advantage of communication technologies such as videotapes, television programming, announcements for CRADA opportunities, and new relationships with trade associations. Specific activities that were planned for FY 1995 to enhance the Laboratory's industrial awareness, with a focus on the Pacific Northwest region and local Tri-Cities area, included

- organizing a technology trade fair focused on technologies suitable for local commercialization

- promoting available technologies and technologies licensed

- establishing an Entrepreneurial Leave of Absence Program to facilitate start-up of new, local firms.

In addition, feedback on the effectiveness and value of the Laboratory's technology transfer is obtained through various mechanisms, such as the Small Business program's use of written surveys to obtain metrics on the effectiveness of our outreach and value provided. Other measures are more informal due to budget constraints in conducting surveys. The value provided to Laboratory customers was thoroughly evaluated as part of the Achieving a Competitive Edge (ACE) Program. This included technology transfer benefits to industry and DOE mission areas.

As appropriate, we actively participate in DOE-wide and federal government outreach efforts, such as the Bobbin Show, NASA 2003, Society of Automotive Engineers, and other forums for presenting Laboratory technologies.

We have a Pacific Northwest Initiative that is designed specifically to enhance recognition of the Laboratory as a valuable technical asset to industries located in the Pacific Northwest region. This Northwest strategy begins with the establishment of formal relationships with the five states and two provinces of the Northwest. Working with these units of government, principally their agencies for commerce and economic development, joint strategies will be constructed to provide support to the development of industries and industrial opportunities in key economic sectors. To support and implement these strategies, the Laboratory will work closely with the industry associations and other science and technology entities designated by the states and provinces as representing these sectors to provide access to direct and leveraged resources where they are most needed.

The kinds of support available from the Laboratory may range from providing selected assistance to small businesses considered critical to the expansion of a given sector to working with the industry association to find opportunities to participate in the procurements and other opportunities available at the Hanford Site. Finally, our strategy for the Northwest includes developing relationships with the key firms in each of the sectors considered critical to the economic development objectives of the Northwest states and provinces. This will mean constructing formal relationships with firms such as Boeing in Washington State and Intel in Oregon. These relationships will be used to promote direct and rapid access by these firms to the resources and facilities of the Laboratory, including access to leveraged partnerships with the Department of Energy where such partnerships can be demonstrated to be in the best interests of all parties.

Significant measures have been taken in the implementation of this Northwest strategy. At present, Battelle and the Department of Energy have signed Memorandums of Understanding (MOU) and Letters of Intent with three of the states and British Columbia expressing the areas of mutual interest and collaboration. The Laboratory is presently in negotiation with all five states and both British Columbia and Alberta on a "Master" MOU and is exploring with the Idaho National Engineering Laboratory its interest in participating in these agreements. The Laboratory is also discussing with the Los Alamos National Laboratory the expansion of these MOUs to encompass the western portion of America from Mexico through Canada to Alaska. In addition, the Laboratory has established formal relationships with the associations of environmental firms, the software association, the biotechnology association, and other industry associations across the Northwest. The Laboratory is also in discussions with key individual firms in these sectors to explore areas in which the Laboratory can be of assistance.

To date, the relationships established with the states and provinces have led to the introduction, at Hanford, of dozens of regional environmental technologies. British Columbia firms alone, have succeeded in establishing contracts with or negotiating agreements for the test and demonstration of 18 innovative environmental technologies. While much of the emphasis to date has centered on environmental technologies and the opportunities to leverage the investment in cleanup at Hanford, new opportunities for economic growth in biotechnology, medical technologies, and energy are being pursued.

Pacific Northwest National Laboratory believes that awards and recognition are an important element of our outreach strategy, as well as being a means of recognizing and encouraging our research staff. For example, the 


\section{R\&D 100 Award Winner}

Real Time Ultrasonic Imaging System-This ultrasonic camera can be used to detect, image, measure, and evaluate flaws in composite materials. It currently is used to verify the integrity of components in high-performance aircraft. Material inspections can be done up to 10 times faster than other methods. The new technology likely will lead to lower aircraft production costs and increased safety for users.

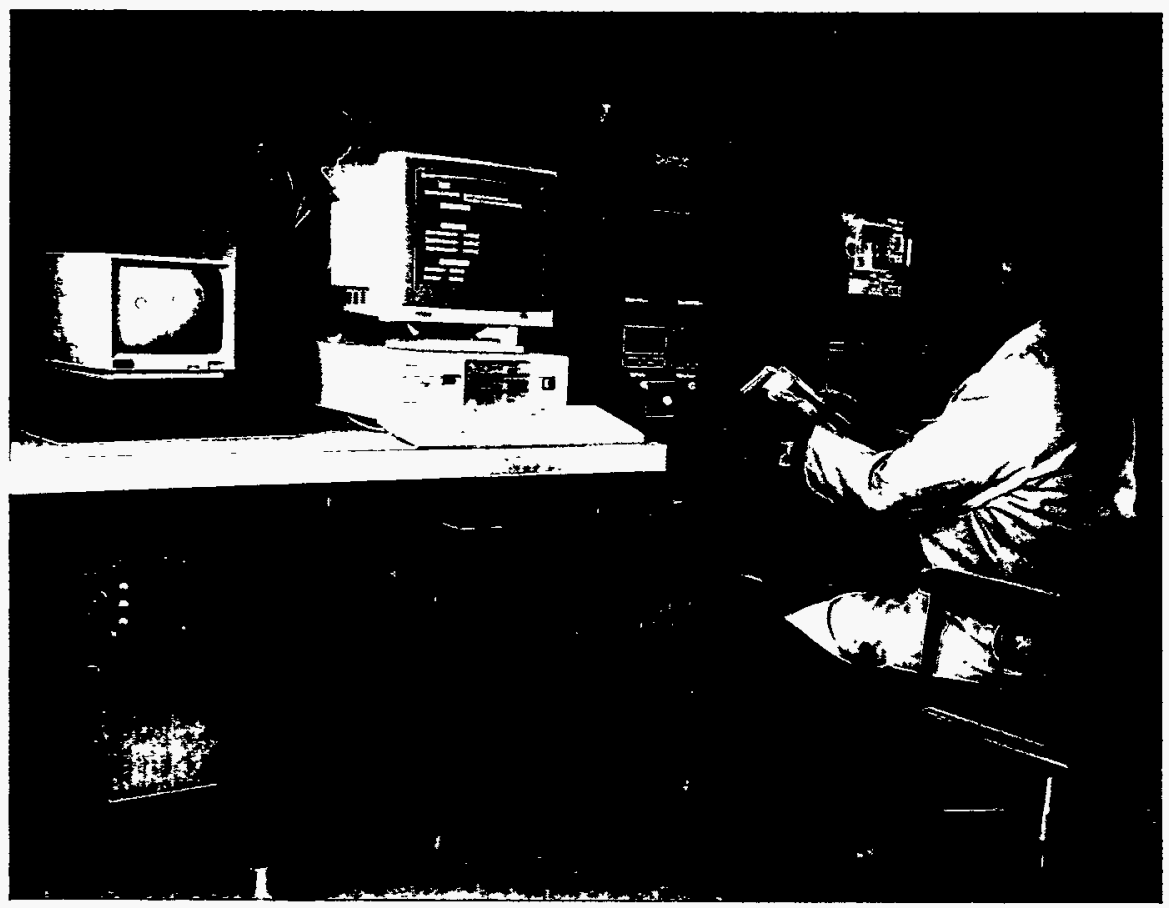

R\&D 100 awards program, sponsored by $R \& D$ Magazine, is a national peerreviewed competition that recognizes the 100 most significant new technological products of the past year. Between 1969, when the first the Laboratory candidate was entered, and 1995 the Laboratory has won 30 awards. Typically, announcement of these awards has led to several hundred inquiries from potential industry partners interested in commercially applying the award-winning technology.

The technologies for which the Laboratory won R\&D 100 awards in 1995 are described below:

- Electrical Remediation at Contaminated Environments (ERACE)-This quick and inexpensive technique produces steam in situ to boil away contaminants in the ground without requiring excavation or soil pretreatment. Soils that would otherwise require years to clean can be cleaned in weeks using simple venting methods.

- Real Time Ultrasonic Imaging System-This ultrasonic camera can be used to detect, image, measure, and evaluate flaws in composite materials. It currently is used to verify the integrity of components in highperformance aircraft.

- Microwave Plasma Continuous Emissions Monitor-This monitor detects hazardous trace materials emitted from smokestacks.

The Laboratory continues to lead the competition in the Federal Laboratory Consortium's Award for Excellence in Technology Transfer. The FLC award recognizes "uncommon creativity" and initiative in transferring technology that provides significant benefits to private industry or state and local governments. The competition is open to all of the federal laboratories-approximately 400-representing 14 federal agencies. Since 1984, the first year of the competition, Pacific Northwest National Laboratory has won 29 awards, more than any other federal laboratory and almost twice as many as any other DOE Laboratory. In 1995, the Laboratory received three awards, which are described below:

- Ultrasonic Microstructural Analyzer-This award was received for the successful transfer of the R\&D-100 award winning technology to practical application in an automotive manufacturing plant. An initial commercial prototype of the technology is now undergoing shakedown testing in an industry partner's components manufacturing plant. Several vendors have expressed interest in developing a new commercial product based on the analyzer and negotiations are on-going.

- Cooled, Optically Stimulated Luminescence (COSL) - The COSL process measures radiation in dosimeters and other thermoluminescent materials. In the process, a dosimeter is cooled to the temperature of liquid nitrogen and then exposed to ultraviolet light. As the dosimeter warms, it gives off visible light similar to thermoluminescent dosimeters. Dosimeters can be re-read, and a greater variety of packaging is possible because no heat is involved. The COSL technology has been licensed to a commercial vendor and efforts to manufacture a commercial version of the instrument are under way.

- Scout-A practical and economically attractive, portable, highly sophisticated radiation analyzer. It is a hand-held, battery-operated instrument that makes a sophisticated characterization of radiological isotopes without requiring laboratory analysis. This technology has been licensed and is in commercial 
production. The first commercial unit was sold in April 1993. To date, approximately 60 units have been sold to government and industrial (primarily electric utility) customers.

\section{Providing Technical Assistance and Collaborating with U.S. Industry}

This objective has two major features: 1) managing the delivery of technical assistance to the private sector (especially to small businesses) and to state and local governments, and 2) increasing collaboration between Pacific Northwest National Laboratory staff and their counterparts in industry and universities. As a national laboratory, we believe that fulfillment of this objective is the most important element of efforts to assist U.S. industry. Activities to achieve this objective fall into six major categories: technical assistance, use of facilities, personnel exchanges, CRADAs, major industry partnerships, and reimbursable work for industry.

\section{Technical Assistance}

Technical assistance is defined as short-term efforts focused on assisting companies, local governments, or trade organizations with specific technical problems. The Laboratory continues to expand its technical assistance with U.S. firms, especially those in the Pacific Northwest. Our most recent expansion is the creation of an Office of Small Business Programs. This office is managed by a full-time Laboratory staff member with research and industrial experience, who serves as a window to the Laboratory and DOE laboratory system capabilities. The office works with the region's small- and medium-sized businesses, with emphasis on women-owned and minorityowned businesses, to help them launch new commercial products, expand their production, address key technical problems, and be more competitive in the commercial world.

A key service that we can provide a small- or medium-sized business is up to 4 days of a researcher's time to address specific problems. This activity is largely supported with funds supplied by the DOE Office of Energy Research - Laboratory Technology Transfer Program, which is described in more detail below. Laboratory staff members are often able to provide valuable solutions to a small firm within the allotted resources. We are seeing a greater use of the program as outreach to small- and medium-sized businesses is expanded in the Hanford Economic Transition Program.

Since the creation of the Office of Small Business Programs in April 1994, 100 small businesses (including 19 that are owned by minorities or women) have been granted requests for technical assistance. Of these firms, approximately half are located within 60 miles of the Laboratory and all but a few are located elsewhere in the Northwest region. The combination of strong demand for assistance from small businesses and finite funding from the ER-LTT Program and DOE-RL will result in technical assistance funding running out for FY 1996 in about June 1996.

The Laboratory's Office of Small Business Programs also assists small businesses in applying for research grants under the SBIR and STTR programs. Assistance for these programs consists of notifying firms of relevant topics that are available, providing samples of winning proposals from the past to use as models, and locating researchers at the Laboratory with whom small businesses can collaborate. In addition, we will assist small firms by making available unique equipment at the Laboratory, special analytical services, literature searches, and, in some cases, may loan equipment. Traditional technology transfer mechanisms such as CRADAs, staff exchanges, and licenses, are also used.

This office is closely coordinated with the Small Business Development Centers located at Washington State University and the University of Oregon, which have offices in every community college in the states of Washington and Oregon. The Office of Small Business Programs also coordinates with the Business Assistance Center at the
Washington Department of Community, Trade, and Economic Development; the Washington Technology Center; the Tri-City Economic Development Council; the Oregon Economic Development Department; and the Advanced Science and Technology Institute in Oregon. This coordination allows all of the organizations to leverage their respective resources and provide a more complete service to the region's businesses.

An example of the successful implementation of this coordinated approach was the joint conduct of three technology transfer workshops for minorityand women-owned businesses in Washington and Oregon states. At the workshops, small businesses learned about technology transfer and procurement opportunities available in the federal sector, as well as sources of technical and business support that are available in each state. All of the firms that have attended the workshops have indicated that the workshops met or exceeded their expectations. The Laboratory's role in this network of support for small business is to serve as the source for technology and technical assistance.

To boost local job creation and economic productivity, the Office of Small Business Programs in cooperation with TRIDEC, hosted a "Technology Fair" where local firms learned about Laboratory technologies that are particularly suitable for commercialization by small businesses. Other activities of the Office in FY 1996 include providing support to start-up businesses that participate in the Laboratory's new Entrepreneurial Leave of Absence Program, working with TRIDEC and other local organizations to recruit new firms into the area, and expanding technical assistance and other programs to include small businesses from Idaho and Montana.

\section{Use of Facilities}

Pacific Northwest National Laboratory has two DOE-designated user facilities - the Fitzner/Eberhardt Arid Lands Ecology Reserve and the DOE Research Aircraft Facility-which are primarily used by academic and 
DOE laboratory researchers. In addition, the Laboratory has extensive specialized equipment and facilities that have been used by industry to address specific technical problems. Laboratory equipment was used during early development, for example, to test and shape a titanium-based composite manufactured by a local company. The composite is now being used in numerous products ranging from wheelchairs to golf clubs. A catalogue of these facilities and equipment has been prepared for distribution to firms interested in using them.

The Laboratory is also developing specialized equipment at its facilities that can be used by industry partners. This equipment includes a superplastic forming press, a materials extrusion facility, a materials coating facility, and a fluid dynamics laboratory. In addition, the EMSL is under construction (see page 32 in Science and Technology). We intend this facility to be a major DOE-designated user facility. Collaborations between industry and Laboratory technical staff who will eventually occupy the EMSL are already under way.

\section{Personnel Exchanges}

Nearly all of the Laboratory's formal personnel exchanges are conducted with support from the ER-LTT Program. The Laboratory has participated in personnel exchanges with industry and universities since the program was initiated in 1985. Exchanges are proposed whenever interests, objectives, and schedules coincide. These exchanges have brought representatives to the Laboratory from approximately seven different companies and one university. In addition, we have sent eight of our staff members to industry. The following personnel exchanges were conducted in FY 1995:

- Barnard Engineering, Great Falls, Montana

- Landauer Inc., Chicago, Illinois

- Allied Technology Group, Richland, Washington

- Golden Technologies, Golden, California
- General Motors Corp., Detroit, Michigan

- Catalysts Inc., Louisville, Kentucky

- Dynamax Inc., Houston, Texas

- Gas Research Institute, Chicago, Illinois

- Kaiser Aluminum, Spokane, Washington

- Oceaneering Space Systems, Houston, Texas, and Richland, Washington

Four of these staff exchanges are with small companies in the Pacific Northwest, and several are with large companies and consortia in the Midwest. This demonstrates the versatility of the staff exchange program. The number of personnel exchanges is expected to decrease in FY 1996, as a result of reductions in the ER-LTT Program budget that supports such exchanges.

\section{Cooperative Research and Development Agreements}

CRADAs provide an important mechanism for Pacific Northwest National Laboratory and other DOE laboratories to assist U.S. industry. At the Laboratory, the creation of CRADAs is energized by a staff that desires to work with industry, and a supportive DOE Richland Operations Office that reviews and approves the Laboratory's proposed CRADAs in a few days to a few weeks.

In the past, the Laboratory has made numerous verbal and written announcements inviting partners to enter into CRADAs, and by the end of 1995 , more than 200 companies had indicated a desire to enter a CRADA with the Laboratory. In FY 1995, we signed and initiated 25 CRADAs, giving a total of 85 signed CRADAs with a combined total value of $\$ 68.8$ million since the authority to enter into CRADAs was received in 1990 . Numerous other partners have been identified and the agreements are awaiting funding. Still other agreements are in various stages of evolution. In FY 1995, we had a record number of amendments to existing CRADAs signed (18). A few of our most recently signed CRADAs and amendments to existing CRADAs are listed below, along with the relevant DOE supporting office.

- NewLight Industries-Develop a laser printer medium and optical print head for a three-dimensional holographic hard-copy printer (funding office is the Office of Energy Research - Laboratory Technology Transfer Program)

- Health Tech Development Inc.Bioactive and porous metal coatings for improved tissue regeneration (funding office is the Office of Energy Research - Laboratory Technology Transfer Program)

- Stirling Technology CompanyMiniaturized Stirling cycle/pulse cooler for nuclear material detection systems (Small Business Innovation Research Program)

- Softdesk, University of Oregon and Trane Company - Integrate a set of computer-based energy design tools with computer-aided design (amendment funded by the Office of Energy Efficiency and Renewable Energy).

Funding support for 58 of the 85 CRADAs currently ongoing at the Laboratory has been provided by the ER-LTT Program. The number of new CRADAs initiated in FY 1996 is expected to decline substantially compared to FY 1995, as a result of budget reductions in the ER-LTT Program, and some ongoing CRADAs may have to be cancelled. The Laboratory is continuing to work with its DOE program sponsors to obtain support for additional CRADAs.

\section{Major Industry Partnerships}

Both prior Congressional legislation and Administration policy have reinforced the importance of establishing major partnerships between DOE laboratories and other organizations as a means to enhance U.S. economic productivity through improved technology transfer. The Galvin Report on Alternative Laboratory Futures reinforces DOE's program of major industry partnerships and the new Office of the Deputy Undersecretary for Technology Partnerships to guide and manage such partnerships. 
Joint research and development activities between DOE laboratories and U.S. industry can be most effective when performed through a coordinated, industry-driven process. Working through industry consortia facilitates fairness of opportunity for industrial participants. Working through an industry-driven agenda ensures that activities are focused on industrial needs. Participation by multiple laboratories provides an opportunity for the unique capabilities of each laboratory to be effectively utilized.

Pacific Northwest National Laboratory has been a key participant in the development of several major industry partnerships that are currently ongoing between the Department, its laboratories, and major U.S. industries. Several specific research projects have already been initiated within the context of these partnerships. Examples of ongoing projects in which the Laboratory is participating include

- AMTEX-Demand Activated Manufacturing Architecture (DAMA) To define, develop, integrate, and deliver an information system structure to be used by all elements of the U.S. textile industry.

- AMTEX-Textile Resource Conservation Project (TReC)-To assist the U.S. textile industry in developing and implementing technology to conserve their resources.

- AMTEX-Sensors for Agile Manufacturing-Develop sensors and feedback controls for flexible, fast, and safe sewing.

- AMTEX-Electronic Embedded Fingerprints-Develop a miniature electronic tag that reports a product's history.

The Laboratory's efforts in these four projects are supported by the ER-LTT Program.

- USCAR-Low Emissions R\&D Partnership-Cylinder Design for Reduced Emission Origins-To bring the latest diagnostic technology to bear on the characterization of dynamic temperatures and processes occurring within an engine cylinder.

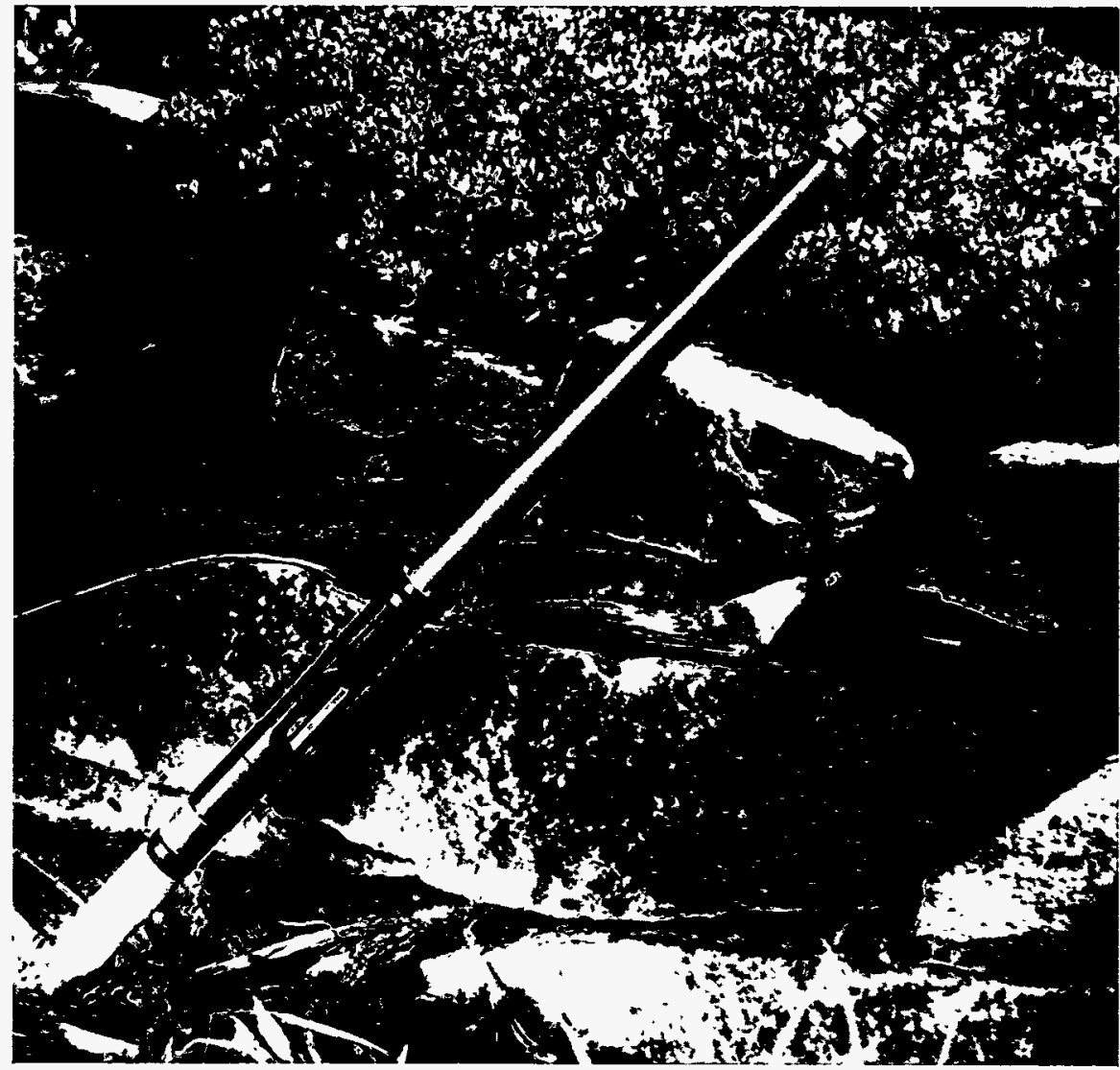

This submersible sensor is being manufactured by a small business located in Washington State. The sensor was commercialized through a license and CRADA. The sensor is used for chemical analysis of deep, remote, or high-pressure bodies of water, such as wells, fresh and marine water bodies, and industrial process flow streams.

\section{- USCAR-Low Emissions R\&D} Partnership—Ultra High Durability, High Temperature Spark Delivery System.

Funding for these two projects with the Low Emission R\&D Partnership is being supplied by the DOE Office of Energy Efficiency and Renewable Energy.

- USCAR-Environmental Research Consortium-Instrumentation for High-Speed Analysis of Vehicle Emissions-To develop new technology for rapid and accurate measurement of low levels of specific compounds contained in vehicle exhaust. Funding for this project is being supplied by the ER-LTT Program.
Pacific Northwest National Laboratory believes that the establishment of successful partnerships with U.S. industry and other external organizations is an essential element of its future. The Laboratory has been a leader in establishing major industry-led partnerships, such as those mentioned above. The Laboratory's interest in such partnerships is a reflection of its long-standing commitment to serving industry and "putting technology to work" that is now receiving so much emphasis in federal and DOE policy.

The present budget situation for major industry partnerships has left the future of such partnerships very much in doubt. In the near future, the Laboratory plans to work with DOE and its industry partners to maintain support for its existing major partnerships (e.g., 
AMTEX and USCAR). Efforts to develop new major partnerships will temporarily be put on hold, even though the Laboratory continues to believe that such partnerships are a major element of its future.

\section{Reimbursable Work for Industry}

Reimbursable work for industry is research and development conducted by Laboratory staff that is fully funded by industry. As such, this represents perhaps the purest form of industry-led activity involving the laboratories. At the Laboratory, nearly all such work is conducted under the Use Permit, which is a unique partnership between Battelle and the DOE that has served industry and the national interest well during its more than 25 years of existence. It also draws upon the skills and capabilities resident in Battelle's private commercial/industrial business.

The Laboratory's strategy with regard to reimbursable work for industry is to conduct such work when it can meet the needs of our industry partner better than the use of other mechanisms, such as CRADAs. Examples of situations where this could occur include projects with extremely short time horizons, projects where obtaining cost-shared federal funding is difficult or impossible, and projects where the industry partner desires to maintain the confidentiality of project results for more than the 5 years allowed by the CRADA agreements. In FY 1994, the Laboratory received approximately $\$ 17.5$ million in funding for such projects, and 74 new projects were initiated with a total contract value of $\$ 7.8$ million. Comparable figures in FY 1993 were $\$ 15.3$ million in funding, and 78 new projects initiated. We believe that the increased interest from industry in using the laboratories to improve their competitiveness will increase the level of reimbursable work for industry in the future.

\section{Commercialization of Intellectual Property}

This element of our strategy accelerates technology development throughout the Laboratory and rapid deployment to industry. It is a means by which we add value to Laboratory-developed technology by enhancing the potential for our industry partners to apply such technology in the commercial world. It also provides an indication of the uniqueness that the Laboratory can bring to bear on addressing a specific industry need. Finally, it provides an opportunity for both the federal government and Battelle to have part of the funds that they have invested at the Laboratory returned for supporting future efforts.

Our philosophy is to commercialize selected technologies as rapidly and broadly as possible. The most frequently used method is licensing. A license agreement is normally nonexclusive unless this would impede commercialization. This strategy allows the Laboratory to have multiple licenses for some technologies. Where an exclusive agreement is required, the scope of a license is limited by fields of use and territory to achieve the broadest benefit of the technology.

In FY 1994, efforts in this area resulted in more than 23 new licenses for U.S. industry for commercial application. Plans to accelerate and expand the commercialization opportunities include the strategies outlined above, i.e., enhanced industrial awareness of the Laboratory as a technical resource and continued development of our technologies through CRADAs and the Use Permit. Also, the Laboratory is taking additional steps to identify potential intellectual property early in the invention process, to evaluate its potential value, protect selected high-value inventions, and encourage their early development. The technology transfer staff works closely with research staff to encourage this early identification and facilitation of valuable ideas.

During 1994, key licenses were signed with U.S. industry. Included is an exclusive worldwide license with Landauer, Inc. for a technology called Optically Stimulated Luminescence Dosimetry. The technology, which measures radiation using materials that luminesce when stimulated with unique beams of light, originally resulted from DOE-sponsored work. Landauer and the Laboratory have collaborated since early 1992 to develop and evaluate the commercial potential of the new technology, which received an $R \& D 100$ award in 1992 and an FLC award in 1995.

A license agreement was signed with a small business for the use of the Laboratory's polymer coating technology for making lithium batteries for portable electronic devices, electric vehicles, and automotive batteries. The company has also signed an Option Agreement to include the use of the technology in the manufacture of cellular telephones, portable computers, pagers, two-way radios, portable audio and video equipment, and portable tools and appliances. Development activities are expected to be conducted over the next several years before final product induction.

An exclusive license agreement for a Laboratory technology related to a new ultrahigh resolution microscope was signed. Consideration for the royaltyfree license is a $\$ 200,000$ microscope for laboratory use. A CRADA with the licensee is on-going and additional licenses related to extensions of the technology are likely.

An ion selective electrode technology incorporating an exceptionally stable reference module, electronics, and submersible sensing electrode into a compact, portable, and inexpensive package was licensed to Instrumentation Northwest, a small company located in Bellingham, Washington. The technology effectively takes chemical measurements in wells, water bodies, and industrial process streams. It eliminates the time and expense of conventional sampling and analysis, can be set up for one or several chemical species, and is adaptable for nonchemical measurements such as temperature and conductivity.

Pacific Northwest National Laboratory is expanding its comprehensive strategy for evaluating and protecting intellectual property targeted for commercialization. The Intellectual Property Protection Committee develops detailed plans for new technologies selected from invention reports submitted by staff members. We have detailed processes to evaluate inventions 


\section{Technology Transfer Effort}

\section{(Budget Authorization \$ in Thousands)}

\begin{tabular}{lrrrrrrrr} 
& $\mathbf{1 9 9 4}$ & $\mathbf{1 9 9 5}$ & $\mathbf{1 9 9 6}$ & $\mathbf{1 9 9 7}$ & $\mathbf{1 9 9 8}$ & $\mathbf{1 9 9 9}$ & $\mathbf{2 0 0 0}$ & $\mathbf{2 0 0 1}$ \\
\hline Outreach Activity $^{(\mathbf{a})}$ & 1,500 & 1,500 & 1,400 & 1,300 & 1,400 & 1,500 & $\mathbf{1 , 5 0 0}$ & 1,500 \\
Patent/Licensing Activity(a) & 2,600 & 2,500 & 1,400 & 1,300 & 1,300 & 1,300 & 1,300 & 1,300 \\
CRADA Funding_Federal: & & & & & & & & \\
Office of Energy Research (b) & 16,000 & 7,800 & 3,500 & 3,700 & 4,500 & 5,000 & 5,000 & 5,000 \\
$\quad$ Other Federal & 1,500 & 800 & 800 & 1,000 & 1,500 & 3,000 & 4,000 & 5,000 \\
CRADA Funding_Private Industry & 100 & 400 & 200 & 300 & 400 & 500 & 500 & 500 \\
Total CRADA Funding(c) & $\mathbf{1 7 , 6 0 0}$ & $\mathbf{9 , 0 0 0}$ & $\mathbf{4 , 5 0 0}$ & $\mathbf{5 , 0 0 0}$ & $\mathbf{6 , 4 0 0}$ & $\mathbf{8 , 5 0 0}$ & $\mathbf{9 , 5 0 0}$ & $\mathbf{1 0 , 5 0 0}$
\end{tabular}

Staffing (Full-Time Equivalents)

$\begin{array}{lrrrrrrrr}\text { Outreach Activity } & 5 & 6 & 6 & 6 & 6 & 6 & 6 & 6 \\ \text { Patent/Licensing Activity } & 18 & 18 & 15 & 15 & 15 & 15 & 15 & 15 \\ \text { CRADA Activity(d) } & 7 & 6 & 3 & 3 & 3 & 3 & 3 & 3 \\ \text { Total Staffing } & \mathbf{3 0} & \mathbf{3 0} & \mathbf{2 4} & \mathbf{2 4} & \mathbf{2 4} & \mathbf{2 4} & \mathbf{2 4} & \mathbf{2 4}\end{array}$

(a) Represents internal and external funds budgeted for crosscutting technology transfer activities, including labor and other costs.

(b) Includes CRADAs performed with institutional support funds, spinoff CRADAs, and The AMTEX Partnership. A significant part of The AMTEX Partnership CRADA funding in FY 1994 was utilized by other DOE-ER laboratories. In FY 1995, AMTEX Partnership funding was sent directly to the laboratories involved and we expect this trend to continue.

(c) Represents external funds in support of CRADAs from federal and private organizations, excluding in-kind contributions.

(d) Represents staff developing and negotiating CRADAs, most of which are supported by external funds.

(e) Represents all Pacific Northwest National Laboratory staff conducting crosscutting technology transfer activities.

\begin{tabular}{|c|c|c|c|c|c|}
\hline & \multicolumn{3}{|c|}{$\begin{array}{l}\text { Licensing Income and Use } \\
\text { (Budget Authorization \$ in Thousands) }\end{array}$} & \multicolumn{2}{|l|}{-} \\
\hline & 1994 & 1995 & 1996 & 1997 & 1998 \\
\hline Number of New Licenses & 280 & 285 & 290 & 295 & 300 \\
\hline License Income & 380 & 240 & 300 & 500 & 800 \\
\hline $\begin{array}{l}\text { Use of Income } \\
\text { Invention Administration } \\
\text { Scientific or Applied R\&D } \\
\text { Awards } \\
\text { Education/Training } \\
\text { Total }\end{array}$ & $\begin{array}{r}230 \\
100 \\
50 \\
0 \\
380\end{array}$ & $\begin{array}{r}100 \\
90 \\
50 \\
0 \\
240\end{array}$ & $\begin{array}{r}135 \\
110 \\
55 \\
0 \\
300\end{array}$ & $\begin{array}{r}150 \\
285 \\
65 \\
0 \\
500\end{array}$ & $\begin{array}{r}200 \\
520 \\
80 \\
0 \\
800\end{array}$ \\
\hline
\end{tabular}


based upon both technical merit and commercial potential. We are currently working on improving the speed and communication associated with our patent evaluation processes after an invention report is submitted for evaluation. Patenting, including foreign coverage, has increased significantly in the past several years from an annual average of 10 U.S. applications to 35 per year. From 1989 to 1994 , the Laboratory received 109 patents.

The Laboratory has experienced a general upward trend in patent and software license agreements in recent years. Some inventions are broad enough to be licensed for several fields of use. Royalties generally lag behind the licensing activity but follow a parallel trend. The lag occurs because royalties depend upon commercial development of the product and product sales. Successful introduction of the product into the market usually takes several years.

Contributing to the significant growth in licenses has been the Laboratory's software technology transfer program to commercialize and distribute federally sponsored software. The Laboratory has developed a systematic process to evaluate software for commercialization potential. If approved for commercialization, the software is copyrighted to protect the interests of the government and the Laboratory. Software which is not commercialized can be released outright for the public benefit. An important objective of software technology transfer is to reinvest the income to make the process financially sustainable.

An integral component of software commercialization is leveraging the resources and expertise of business partners (licensees) to effectively market and distribute Laboratory-developed software. The major licensee selection criteria are technical expertise and reputation, compatibility of their product line and industry focus with the software, ability to market and distribute software as evidenced by their sales/marketing channels and track record, size and type of customer base, and the overall terms and conditions of the partnership agreement. Joint development projects with industry are also undertaken to address market requirements. At the Laboratory, collaboration with partners is an ongoing activity that continues long after the contract is signed. Customer feedback is important as future versions of software are released.

An example of effective partnering is licensing of the NUCLIDES software to $E G \& G$ Ortec, a major leader in the nuclear instrumentation market. EG\&G intends to incorporate this code into their suite of software products and market it worldwide.

We have improved distribution of our software products through several channels: WindowChem Software, Inc., which obtained rights to nonexclusively distribute ReOpt ${ }^{\mathrm{TM}}$, MultiMedia Environmental Database Editor (MMED), and SNYTH (Synthetic Gamma Ray Spectroscopy); Battelle Press, which targets colleges and universities and specific industry segments for $\mathrm{ReOpt}^{\mathrm{TM}}$ and MEPAS $B$; ESTSC for government users and contractors. Over the next year, it is expected that energy efficiency and data visualization software will be commercialized.

\section{Laboratory Directed Research and Development Program}

The relevance and value of a DOE multiprogram laboratory lie in its ability to apply science and technology to national needs that fall within the business areas of the DOE. The increasing complexity of these needs and the inadequacy of conventional approaches demand that creativity and innovation underlie scientific and technological efforts. In addition, new ideas and opportunities frequently occur at a faster pace than can be anticipated or adopted in the federal budget process.

A national laboratory must establish and maintain an environment in which creativity and innovation are encouraged and supported if it is to fulfill its objectives and remain viable in the long term. For these reasons, external reviews of the DOE multiprogram laboratories have consistently recommended that laboratory directors be allowed to allocate a percentage of their operating budget to support discretionary research and development projects.

DOE Order 5000.4A establishes DOE's policy regarding Laboratory Directed Research and Development (LDRD) at its multiprogram laboratories and authorizes them to allocate up to 6 percent of their operating budgets to LDRD. LDRD is "...research and development work of a creative and innovative nature which is selected by the director of a laboratory, or his/her designee, for the purpose of maintaining the scientific and technological vitality of the laboratory and to respond to scientific and technological opportunities."

\section{Program Administration and Management}

Laboratory Directed Research and Development at Pacific Northwest National Laboratory is funded through an overhead account that is part of the General and Administrative overhead that is applied to all operating funds received under the 1830 Contract. Formal proposals in an authorized format are required for each LDRD project.

Decisions regarding funding levels for the LDRD account are made by the Laboratory director. Primary responsibility for allocations to individual LDRD projects within these accounts rests with the Laboratory Technical and Strategy Councils (composed of technical staff and senior line managers, respectively), who assist the Director in determining these allocations.

All projects are reviewed for technical merit by line managers and/or scientific staff, and in some cases by external peer reviewers. Written guidance pertaining to the criteria and guidelines for LDRD projects provided in DOE Order 5000.4A are widely distributed to Laboratory staff and managers through a formal LDRD Guide and other correspondence. Adherence to the criteria of $5000.4 \mathrm{~A}$ is further ensured through reviews by the LDRD 


\section{Laboratory Directed Research and Development}

(Budget Authorization \$ in Millions)

\begin{tabular}{lcccc} 
Current Program & 1993 & 1994 & 1995 & $\mathbf{1 9 9 6}^{(\text {a) }}$ \\
\hline Total LDRD Funding & 11.8 & 13.8 & 14.9 & 15.5
\end{tabular}

(a) The estimated amount for FY 1996 is a forecast maximum which DOE has approved.

Office. Individual projects are usually limited to $\$ 1$ million in total funding and $\$ 500,000$ in a single fiscal year. The Director of Finance is responsible for financial oversight of the LDRD Program. Accountability for individual LDRD projects rests with the principal investigators conducting the projects.

\section{Scientific and Technical Investment Areas}

Pacific Northwest National Laboratory's LDRD Program supports new and innovative projects throughout the Laboratory; however, the Laboratory's core competencies are targeted for emphasis. These competencies and the percentage of the total LDRD funding that each received in FY 1994 (the most recent year for which data are available) follow:

- integrated environmental research (49 percent)

- process science and engineering (30 percent)

- energy systems development (11 percent).

While emphasis is placed on the above core competencies, LDRD funding also is allocated for projects judged to have high scientific/technical merit that are independently proposed by individual researchers or small, multidisciplinary teams within our research centers.

The Laboratory plans to continue to focus the majority of its LDRD investments in the development of scientific and technical areas that underlie the three core competencies. Examples of such areas include the environmental sciences, biological sciences, computer and information sciences, chemical and materials processing, and energy technology.

\section{Selected Highlights of LDRD Projects}

In FY 1994 (the most recent year for which data are available), 161 LDRD projects were selected for support through the Laboratory's LDRD project selection process. Selected highlights of the results of these projects follow:

- A project entitled, "Chemical Sensors" resulted in the development of new Suspended Gate Field Effect Transistor (SGFET) sensors for detection and measurement of environmental contaminants. A number of new chemically sensitive layers (a critical element in determining sensor accuracy and sensitivity) for the SGFET and other types of sensors were also developed.

- Research on "Integrated Regional Climate Change" resulted in the development of an integrated physically based modeling system that spans the wide range in scales required for assessment of climate impacts on one particularly vulnerable component of the climate system, namely surface hydrology. The modeling system consists of one-way coupled physically based process models of the atmosphere and watershed hydrology.

- A project entitled, "Synthesis of SiteSpecific Lesions" demonstrated that DNA containing specific 4-AcHAQO adducts amenable to molecular and structural analysis can be produced. Methods for ligation of short (15 to 25 base pair) modified oligonucleotides to larger DNA fragments for structure and function studies were also developed in the project. These developments represent an important first step to characterizing the mechanisms whereby modification of DNA by chemical carcinogens are converted into mutation and thereby initiate tumor formation.

- Studies of "Advanced Catalysts and Catalytic Processes" have identified sulfated zirconia catalytic materials with superior properties for n-butane isomerization. When fully developed, this solid acid catalyst could offer significant energy and environmental benefits compared to the current catalysts used in butane isomerization.

- A project entitled "Synthesis of Advanced Coatings" resulted in significant improvement in the deposition conditions for $\mathrm{SnO}_{2}$ thin films by increasing the $\mathrm{pH}$ at which the films can be grown. In addition, the fundamental mechanisms determining biomimetic film growth kinetics were identified. These developments are critical to determining appropriate processing applications for these advanced coatings.

- A project on "Superplastic Forming Aluminum Developed Multiwall Exhaust" resulted in significant advances in the ability to form complex shapes with superplastic forming technology. Full development and application of this technology could significantly reduce 
vehicle weights and vehicle energy use by facilitating the widespread use of superplastic forming.

More extensive information on the technical highlights of Pacific Northwest National Laboratory's LDRD Program is reported in the Annual Report on Laboratory Directed Research and Development. The FY 1995 Annual Report is currently being prepared for submission to DOE in February 1996.

\section{Work for Other DOE Sites}

Pacific Northwest National Laboratory will continue to provide expertise for solving varied problems at a number of DOE sites. Technical services will continue to focus on waste management and site cleanup, as well as numerous institutional areas such as safety and security. As previously reported, the Laboratory has supported the environmental restoration of the DOE Laboratory for Energy-Related Health Research near Davis, California. The project consists of supporting decommissioning of five buildings and remediation of soils and groundwater. Major accomplishments include decontamination and decommissioning of four buildings and initiating a comprehensive RI/FS for the site. This activity has been a model for other decontamination and decommissioning activities and has saved DOE several million dollars by streamlining the activities. We are also identifying mature cleanup technologies from foreign sources and delivering them to DOE sites in a project supported out of Ames Laboratory. To date, about 100 technologies have been identified from the private sector and 19 are now at work within the DOE complex.

As the primary developer of waste vitrification technology, we continue to transfer the technology for treatment of nuclear and other hazardous wastes at DOE sites. The Laboratory is providing direct support to other sites in areas such as high-level waste vitrification processes, waste form qualification, and innovative waste treatment technologies. Since project inception in the early 1980 s, we have been a technology partner providing major support to the West Valley Demonstration Project in New York State which is scheduled to begin radioactive operation in 1996. In support of the Oak Ridge National Laboratory, tests have been conducted and are continuing to support decisions on whether to in situ vitrify buried lowlevel and transuranic waste in the onsite trenches. Two, multi-hundred-tons treatability tests were planned to be conducted in a radioactive liquid waste seepage pit during FY 1995 in support of the Record of Decision for closure of sites at Oak Ridge. This test is the culmination of 9 years of collaboration between Pacific Northwest National Laboratory and Oak Ridge National Laboratory targeted to remediation of Oak Ridge National Laboratory pits and trenches. In addition, the Laboratory is providing highwaste-loading glasses for multiple programs managed at INEL and other sites. The work includes developing glasses that are processable over a wide range of compositions to allow treatment of wastes previously thought not suitable for vitrification.

Pacific Northwest National Laboratory is providing support to the Waste Isolation Pilot Plant (WIPP) by determining the corrosion and gas generation characteristics of low-carbon steel in the WIPP environment. Another related project is determining the solubility of actinide compounds in complex brines. As part of the DOE-DOD Strategic Defense Environmental Research Program at Sandia National Laboratories, we are providing data system development and experimental design for the unmanned aircraft component of the Atmospheric Radiation Measurement Program. This work has led to the first applications of unmanned aircraft for atmospheric research.

In support of Knolls Atomic Power Laboratory, we are assessing irradiated subcapsules to determine their mechanical, thermal, and physical properties. To date this project has provided key engineering design data for their advanced naval reactor development.

The Laboratory will continue supporting other DOE sites over the next several years as we transfer some of the new technologies being developed for the Hanford Site. In the long term, the level of support is expected to be relatively flat.

\section{Work for Others}

In addition to contributing to the implementation of the DOE Strategic Plan, Pacific Northwest National Laboratory performs work for other federal agencies in selected scientific and technical areas in accordance with DOE polices. Work undertaken for other federal agencies was $\$ 59$ million FY 1995. It is expected to increase slightly. The largest segments of the Laboratory's work for others program are work for the Department of Defense and the Nuclear Regulatory Commission.

A number of other federal agencies fund work at the Laboratory. They include the Environmental Protection Agency, Health and Human Services, the National Aeronautic and Space Administration, and the Federal Emergency Management Agency. The work for these agencies is briefly described below.

\section{Department of Defense}

Pacific Northwest National Laboratory's work for the DOD primarily utilizes the Laboratory's capabilities in electronics and sensors, statistics and applied mathematics, computer and information sciences, materials science, chemical and instrumentation analyses, and integrated technology policy and regulatory analysis. The level of activity for DOD was $\$ 32$ million for FY 1995 and is expected to increase slightly beyond. The Laboratory is currently working in the following technical areas:

- treaty verification and technology assessment

- information sciences and architectures

- advanced materials and process development

- sensors, electronics, and automated systems and their applications

- systems analysis and technology evaluation 
- environmental science and waste technology

- analytical chemistry and radionuclide applications.

The Laboratory has a significant role in the area of treaty verification and technology assessment based on our unique technical skills. This work, in close coordination with DOE, is performed for a number of DOD clients in support of national security needs.

The Laboratory supports Navy, Marine, Army, and Air Force research and development needs for systems integration using the Laboratory's capabilities in information sciences, networking architectures, and software development. The Laboratory develops and evaluates advanced tactical command, control, and communication networks for the Marines and Army. Programs with the Air Force and Army use advanced graphic technologies, automated training systems, expert systems, and advanced workstation technology to simplify and automate operations. Network architectures and distributed computing techniques are developed to meet the unique needs in systems integration projects for the munitions and depot commands of the Army.

We expect to continue providing support to the DOD in advanced materials and process development. The Kinetic Energy Projectile Program supports design, analysis, testing, and development of advanced munitions and manufacturing techniques for the Army's Armament Munitions and Chemical Command. Studies of composite materials and other unique materials are performed for many other DOD clients. Other materials work for the DOD includes developing optical and other coatings technologies that include high-efficiency and selectivity reflective coating, and high-quality coatings of very large-scale optics (currently up to 2 meters).

Sensors, electronics, and automated systems development work is also expected to continue. We are developing sensors for chemical, environmental, and biological measurement, and imaging technology for DOD. Millimeter wave, radio frequency, electrooptical, and fiber optic technologies are being developed for test range instrumentation systems for the Army and missile test systems for the Navy. Ultrasonic and electromagnetic imaging systems to evaluate airframe, munitions, and undersea components are being fielded. A variety of unique robotics for safety testing and specialized repair tasks, such as reverse engineering systems and printed circuit board repair have been developed for the Army. We are developing a telerobotic system for disposal of explosive ordinance for the Navy.

Pacific Northwest National Laboratory provides expert assistance to the DOD in systems analysis, technology evaluation, and statistics. This work includes the technical support for modernization of major weapons production and logistics elements with the Army; evaluation of organization systems, procedures and methods, safety systems, and functioning of joint programs; and the assessments of human factors impacts and training requirements. Industrial base modernization programs evaluate and apply computer-integrated manufacturing in Army production plants, and provide efficient automation technology options to meet specialized maintenance technology requirements of the Army, Navy, and Air Force.

The Laboratory has a program with the Advanced Research Projects Agency for developing medical systems and technologies that improve health care and battlefield treatment of injured. We are working with both DOD and DOE to expand this effort into a broad and integrated partnership that would include all the national laboratories and Army medical centers.

Pacific Northwest National Laboratory is the lead laboratory for energy management for DOE's Federal Energy Management Program (FEMP). Under FEMP, the Laboratory has developed and is deploying a model program for installation-wide energy-system modernization. Software tools and methodological approaches are being developed for rapid assessment of the energy resource potential at each installation. The Laboratory then works with the installation energy manager and local utilities to create demand-side management programs. This approach modernizes federal energy systems with funds from the utility's capacity savings through conservation. The program also is developing a knowledgebased decision guide for operating and maintaining heating plants, and assisting facilities planning using geographic information technology. We maintain world-class capabilities in environmental characterization and analysis of impacts on a variety of ecosystems from arid lands to wetlands and estuaries. All services, most notably the Army Corps of Engineers, seek out Laboratory staff for specific analyses, and for development and synthesis of prevention and mitigation technologies to minimize deleterious environmental impacts.

The Laboratory continues to provide a variety of DOD clients with analytical chemistry support and radionuclide applications development. Very sensitive and high-precision analytical techniques are available at the Laboratory to meet measurement and testing requirements. Historical and unique skills in analyzing radionuclides support specialized DOD needs.

As part of the Chemical Demilitarization Program, Congress has mandated that the U.S. Army provide support to assist the civilian population in dealing with the effects of an event involving the accidental release of chemical munitions. This program is the Chemical Stockpile Emergency Preparedness Program (CSEPP). For the first several years of its development, the Federal Emergency Management Agency (FEMA), acting as agent for the Army and because of its national role in emergency preparedness, managed the program. Under FEMA management, the Laboratory developed several emergency management decision support software systems. The current development activity is the Federal Emergency Management Information System (FEMIS).

The U.S. Army continues as the lead in this program, with continued support from FEMA, directing the development of a computing system which will support state and local emergency 


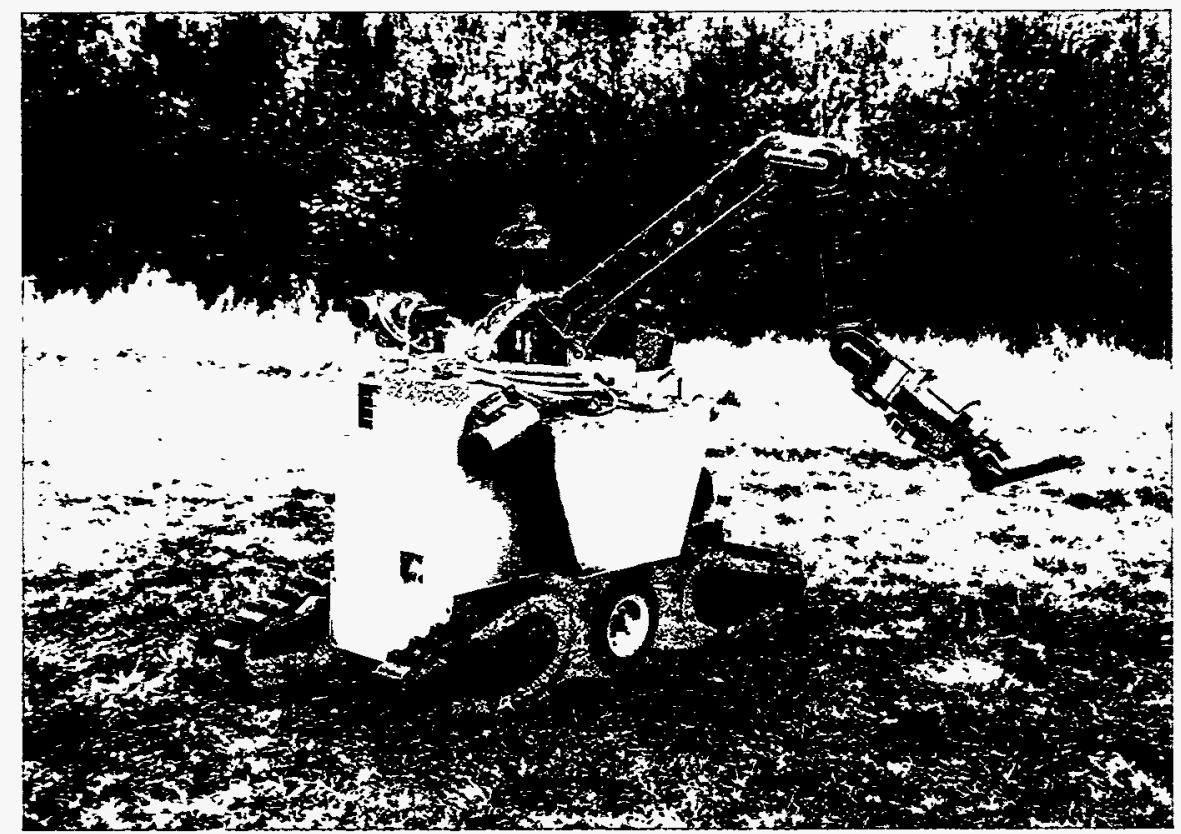

RONS (Remote Ordnance Neutralization System) is teleoperated from a remote operator control station.

management agencies as well as U.S. Army Chemical Storage Depots and FEMA in dealing with CSEPP emergencies. The Laboratory has completed development and delivered the first phase of the FEMIS decision support software system. On May 1, 1995, the Army began fielding the software at the eight storage sites and in the surrounding communities and states.

Follow-on support will take the form of development of individual hazard modules for the system, and extension of the functionality of the software to multiple hazards to position FEMIS as a national emergency management system. Enhancements to basic functionality, and configuration management of the in-place system are expected to continue during its lifetime. This effort would extend approximately 12 years into the future.

FEMIS is currently funded at $\$ 10.2$ million. Follow-on funding of an additional $\$ 3$ million over the next year is expected with a continued level of effort out approximately 10 years. Pacific Northwest National Laboratory established copyright to the software and is in the process of developing other emergency management business with other customers including other federal agencies (DOE, the Forest Service and the Corps of Engineers), as well as states (Oregon, Delaware), and foreign governments (Mexico).

\section{Nuclear Regulatory Commission}

Pacific Northwest National Laboratory's research and technical work supports all of the U.S. Nuclear Regulatory Commission (NRC) program offices, regional offices, and several administrative offices. The work covers all aspects of nuclear safety regulation. The level of activity for the NRC is expected to decrease in future years. For FY 1996, NRC's total budget will be decreased approximately $\$ 50$ million from FY 1995 . This will have a significant impact on the work done at the national laboratories. Estimated level of activity for Pacific Northwest National Laboratory during FY 1996 is $\$ 8$ to $\$ 10$ million. This level is expected to hold during the following years.

Significant activities for the NRC that either reached, or were approaching completion during FY 1995 include the following:
The Standard Review Plan Update and Development Program (SRP-UDP), to update the Standard Review Plan (NUREG-0800) for the Office of Nuclear Reactor Regulation (NRR), will be completed in FY 1996. The Standard Review Plan will be used by NRC staff to review applications for license and/or design certification concerning nuclear power plants or modifications thereto. The Laboratory assisted the NRC with updating NUREG-0800 to reflect the existing agency requirements and guidance and to incorporate review guidance and acceptance criteria for unique technology and the unique application of existing technology in future designs. Following completion of the revised NUREG-0800, a small maintenance effort will begin and is expected to continue for the next few years.

Development of a construction inspection database for the NRR will be completed in FY 1996. This database was developed to manage the NRC's construction inspection programs for future plants. The database, including data input, retrieval, and report generation, is based on off-the-shelf software, making it widely applicable to other projects that need similar schedule managing, tracking, and report generation capabilities.

The Laboratory's role in the Nuclear Plant Aging Research (NPAR) Program for the Office of Nuclear Regulatory Research (RES) will be completed in FY 1996. NPAR addressed issues associated with the reliability of safetyrelated equipment, plant life extension, and license renewal. However, the Laboratory anticipates providing related assistance to the NRC in 1996 and beyond with future license renewal activities including the review and assessment of license renewal applications.

Pacific Northwest National Laboratory support to the Office of Nuclear Regulatory Research in evaluating past reactor operator crew examinations to identify trends and patterns in operators and crew ability on the job was completed in FY 1995. The Laboratory evaluated variables such as 
operator education and experience in an attempt to infer from these variables the effects on operator ability on the job and to compare results to human reliability analysis methods used in current probabilistic risk assessments.

Support to the NRC that will continue or develop over the planning period includes the following.

\section{Office of Nuclear Reactor Regulation}

The Laboratory will continue to provide support to the NRR's Operator Licensing Branch in licensing commercial power plant nuclear reactor operators and senior reactor operators and in evaluating facility operator requalification programs. The Pacific Northwest National Laboratory has provided this support annually to approximately 300 to 400 candidates at 50 sites nationwide, supporting all five regions and headquarters. The Laboratory has 18 fully certified examiners qualified to administer exams in all pressurized water reactors, boiling water reactors, and nonpower/ research reactors. Workload may decline significantly during the next 5 years due to proposed, but not yet adopted, regulatory changes for administration of NRC requalification examinations. The broad background of the operator licensing examiners and their specialized training provides a pool of staff able to support a wide variety of other activities for both the NRC and DOE. The experience of the examiners continues to be beneficial in performing the following types of activities: maintenance and training inspections, risk analysis, risk management, simulator evaluations, emergency preparedness assessments, and individual plant examinations.

Pacific Northwest National Laboratory will continue to support NRR's Division of Reactor Project Management by providing technical assistance in improving work processes, supporting staff reviews of decommissioningrelated activities, and assisting the staff in improving the process to review potentially generic safety-related information. This support includes information technology and applicable training of NRC staff.

The Laboratory will continue to support the NRR's Division of System, Safety and Analysis in the area of commercial in-reactor fuel performance. This work includes performing technical review of vendor and utility submittals on fuel designs, fuel performance codes, and control rod assemblies. An annual summary of in-reactor fuel performance, based on information from the vendors and open literature publications, will continue to be prepared. This summary includes discussions of design trends, failure trends, and other topics of relevance to fuel performance. An increasing role will be to assist the $\mathrm{NRC}$ in onsite audits and inspection of fuel vendors and utilities.

Pacific Northwest National Laboratory will provide assistance to the Office of Nuclear Reactor Regulation in regulatory and licensing activities in the siting and environmental protection areas, including the Environmental Standard Review Plan update and development effort. The Laboratory will provide multidisciplinary managerial and technical expertise to assist the NRC in its licensing reviews for early site permits and operating reactors, and in updating regulatory guidance.

\section{Office of Nuclear Regulatory Research}

Pacific Northwest National Laboratory will continue to provide technical assistance to the RES in support of decommissioning analysis and regulation. Technical analysis and cost estimates are provided for decommissioning licensed nuclear reactor power plants and for licensed fuel-cycle and nonfuelcycle nuclear facilities. The support the Laboratory has provided to the NRC on short-turnaround analyses and addenda to previous decommissioning analysis reports should continue on a task basis. Current and future work for the NRC includes completing the reevaluation of the original pressurized water reactor and boiling water reactor power station decommissioning analyses (NUREG/CR-0130 and NUREG/
CR-0672) and a computer program developed for estimating decommissioning costs; periodic updates of NUREG-1307 to reflect changes in the low-level waste burial site charge schedules; an analysis of the decommissioning of a large sealed-source user facility; and the reevaluation of the earlier fuel-cycle and nonfuelcycle facility reports to reflect current financial and regulatory conditions.

The Laboratory is assisting the NRC with three closely related projects to provide regulatory analysis support for the materials licensee-related activities and power reactor-related activities, to update and revise the Regulatory Analysis Guidelines, and develop a Regulatory Analysis Technical Evaluation Handbook. The Laboratory is also tasked to perform the in-depth technical analyses necessary to support NRC decisions on safety issue resolution. These analyses include probabilistic risk assessments and cost analyses that provide the information for NRC staff to support regulatory decisions.

Nondestructive evaluation projects conducted at the Laboratory for RES have provided the engineering databases to support the NRC's position and policy on regulatory guides, position statements, codes, and regulations. The elements of these projects include 1) studying nondestructive evaluation reliability to determine the effectiveness of in-service inspections; 2) optimizing in-service inspection programs using nondestructive evaluation reliability data, probabilistic risk assessment methods, and fracture mechanics analysis to control risks; 3 ) assessing new nondestructive evaluation techniques and transferring technology to the NRC regional offices and to the utility industry; 4) developing a technical database for fabrication flaws in U.S. reactor pressure vessels for use in remaining life predictions; 5) designing and fabricating a steam generator mockup for regional NRC staff to assess inspection performance at reactor sites; and 6) developing information on computer-based ultrasonic systems to enable NRC staff to understand and audit inspection results. The nondestructive evaluation technologies under 
study at the Laboratory include ultrasonics, eddy currents, and acoustic emission. It is anticipated that when the advanced light water reactor designs proceed, the Laboratory will be involved in similar work for the new designs.

The Laboratory will continue to provide technical assistance to the Office of Nuclear Regulatory Research in support of low-level radioactive waste storage. Such support includes classification, characterization, and assessment of waste streams and activated metals; source terms for performance assessments; and characterization of chelating agents.

The Laboratory plans to continue to support radiation protection projects such as calculating fractional transfer of radionuclides from a pregnant woman to embryo/fetus to be completed in FY 1996, and reconcentration of radioactive material in sanitary sewers to have been completed in FY 1995.

\section{Office of Nuclear Material Safety and Safeguards}

Pacific Northwest National Laboratory will continue to provide technical assistance to the Office of Nuclear Material Safety and Safeguards (NMSS) in the area of regulation of the Gaseous Diffusion Plants at Portsmouth, Ohio, and Paduccah, Tennessee, which are being operated by U.S. Enrichment Corporation (USEC) through a lease arrangement by $D O E$. The Laboratory will assist NMSS in at least three areas: 1) engineering, systems, management controls, and human factors; 2) radiation and chemical safety; and 3) safeguards. Assistance to NMSS on applying nondestructive assay techniques to determine holdup in piping systems began in FY 1995. The key activities for the short term will be to assist the NRC in developing the specific technical and inspection criteria and guidance and assisting with the reviews and inspections. Because the Gaseous Diffusion Plants must be certified annually, this activity is expected to continue during the planning period. This assistance may also take the form of help provided to region staff, Office for Analysis and Evaluation of Operational Data, or other NRC offices, and the Gaseous Diffusion Plants resident inspectors.

USEC is also pursuing development of the Advanced Vapor Laser Isotope Separation (AVLIS) technology to supplement/replace the Gaseous Diffusion Plants for commercial enrichment services. NRC may be expected to ask the Laboratory for assistance in reviewing such a license application in the future.

The Laboratory began providing additional support to NMSS in FY 1995 in the area of chemical safety, security and safeguards, fuel cycle regulatory guides, and international physical protection. This work is expected to continue. Should DOE decide to turn over additional facilities to NRC regulation, opportunities will exist to assist the NRC in reviewing those facilities.

The Laboratory provides technical support to the NRC in drafting environmental impact statements for low-level waste sites. The Laboratory is being considered for a series of environmental assessments at several uranium sites in New Mexico through NMSS.

\section{Other NRC Offices}

Pacific Northwest National Laboratory continues to support the Office for Analysis and Evaluation of Operational Data by refining analytical tools used by the NRC's Emergency Response Organization. New and/or improved models that address cloud shine, modify wind fields, process meteorological forecasts, present results graphically, calculate dose rates, etc., will be added to the RATCHET and RASCAL codes. After the codes are revised, tested, and installed, the Laboratory will train NRC staff in the use of the models.

\section{Environmental Protection Agency}

Pacific Northwest National Laboratory conducts research to assist the Environmental Protection Agency in its central role of environmental regulation in the U.S. The level of activity for EPA was \$2.7 million in FY 1995 and this level is expected to decrease slightly in FY 1996 and beyond. We will continue to conduct a variety of research and development activities to improve the state of knowledge about exposure, impacts, and risk from pollutants on human health and ecological systems. We are conducting research to investigate the causes and effects of global climate change, and we expect that EPA will continue its support for such research in its Global Climate Research Program. The Laboratory is also involved in measuring and assessing the impacts of pollutants on ecosystems including the Arctic, the Everglades, and Pacific Northwest watersheds. We provide technical support to the Environmental Monitoring and Assessment Program (EMAP) by assisting in the design of studies to estimate the current status, extent, changes, and trends in indicators of the condition of the nation's ecological resources on a regional basis. The Laboratory is also developing and demonstrating methodologies and technologies to understand and mitigate risks associated with hazardous materials. The primary areas of research that we will conduct for the EPA include the following:

- modeling and assessment of the environmental impacts of increasing concentrations of trace contaminants in the atmosphere and potential resultant global climate change

- assessing the technologies and economic impacts of selected international strategies to reduce greenhouse gas emissions

- analysis and modeling of hazardous waste transport and fate in soil, water (both fresh and marine), air, and biota

- measurements and mass balance determinations to assess sources and sinks of polychlorinated biphenyls in Lake Michigan

- research, evaluation, testing, development, and demonstrations of alternative or innovative hazardous waste treatment and radon mitigation technologies 
- measurement and analysis of the effects of toxic and hazardous chemicals on terrestrial and aquatic ecological systems, including the marine environment

- support for establishing and operating the Beijing Energy Efficiency Center

- modeling the formation, transport, and impacts of acid rain and evaluation of strategies to control it.

\section{Health and Human Services}

Pacific Northwest National Laboratory's major Health and Human Services program is conducted for the National Toxicology Program. The National Toxicology Program is a program of NIEHS, a component of the National Institutes of Health, which is part of the Department of Health and Human Services. The Laboratory has established a center of excellence in inhalation technology and toxicology for the National Toxicology Program that is consistent with, and complementary to, our long-standing research efforts conducted for the DOE. This program has strengthened our knowledge and capabilities in these areas, which are being applied to research on hazardous wastes.

The National Toxicology Program was established 13 years ago to coordinate and strengthen government activities in characterization of the toxicity of chemicals. The National Toxicology Program is charged with

- broadening the spectrum of toxicologic information on selected chemicals

- increasing the number of chemicals studied, within funding limits

- developing and validating assays and protocols responsive to regulatory needs

- communicating program plans and results to government agencies, the medical and scientific communities, and the public.

The National Toxicology Program has recently initiated toxicology and carcinogenesis studies related to electromagnetic field exposures.
Toxicology-related research is central to the three major agencies of the National Toxicology Program: the Food and Drug Administration, NIEHS, and the Centers for Disease Control's National Institute for Occupational Safety and Health. The total government-sponsored inhalation toxicology program at the Laboratory is currently funded at $\$ 8$ million to $\$ 11$ million per year, of which less than 2 percent has been funded through Work for Others agreements under our operating contract (1830).

During the past decade the National Toxicology Program has established an integrated program for studying chemicals. The program investigates multiple toxicologic endpoints using assay protocols tailored to each chemical. The "traditional" 2-year carcinogenesis bioassay has been strengthened into a comprehensive toxicologic evaluation that provides information not only on a chemical's carcinogenic potential in laboratory animals but also on genetic toxicity; chemical absorption, distribution, metabolism, and excretion; target-organ toxicity; and reproductive effects. Among the endpoints examined in specific cases are neurobehavioral, immunologic, hematopoietic, respiratory, physiologic, and endocrine effects. During the past several years, increased numbers of chemicals have been tested by the inhalation route of exposure because inhalation is a common route by which workers and the general populace are exposed to potentially toxic chemicals. Data acquired from exposures of laboratory animals under controlled conditions are used to assess potential health effects in humans and may be used to set standards for worker protection or to indicate new areas for basic research.

Pacific Northwest National Laboratory has the following primary goals and areas of research for the National Toxicology Program:

- continue to be a dominant inhalation toxicology laboratory by maintaining and improving the technical quality of the research conducted

- broaden the scope of work performed to include greater in depth evaluation of target-organ toxicities, such as pulmonary function, cardiovascular physiology, cell and tissue kinetics, tissue distribution of native and biotransformed chemicals, ultrastructural analyses, and pharmacokinetics studies

- demonstrate to the National Toxicology Program our capabilities in molecular biology

- conduct additional studies with laboratory animals on the developmental toxicity of chemicals following inhalation exposure.

\section{National Aeronautics and Space Administration}

Pacific Northwest National Laboratory is performing work for the National Aeronautics and Space Administration (NASA) in a number of areas related closely to DOE's core technology areas. A continuing program is the development of a Space Station Leakage and Impact Detection System.

Another continuing program is the development and fabrication of radiation dosimetry instrumentation for space applications. This work is being performed for NASAJJohnson. Approximately $\$ 1$ million has been funded to date and it is anticipated that NASA/Johnson may fund a prototype followed by a subsequent system for field test.

NASA is also funding work at the Laboratory for the development of ultralight fabric heat-pipe radiators for space applications. Fabrication has been demonstrated for prototypes and it is estimated that radiator systems based on this concept will be 40 to 60 percent lighter than systems currently being used.

We are also working with NASA on the development of ultralight fabrics to shield space vehicles and Space Station Freedom from orbital debris and micrometeoroids. The fabrics will be used to shield piping, tanks, and other critical components. Joint studies indicate a potential for millions of dollars in cost reductions through use of these fabrics.

Other work for NASA includes a continuing program on monitoring chemical releases from upper atmospheric 
rockets, radiological measurements on the Long Duration Exposure Facility satellite samples, and development of rotocraft simulator standards. We are also planning a future, major collaborative effort with NASA in global change as an extension of our DOE program in this area.

\section{Federal Emergency Management Agency}

Pacific Northwest National Laboratory will continue to support the Federal Emergency Management Agency (FEMA) through the development of automated decision support tools in the areas of emergency planning and crisis management. FEMA's primary focus is in the area of the long-term, multiple-hazard application of the Federal Emergency Management Information System (FEMIS).

The U.S. Army now has the leadership role in directing the development of a computing system that will support state and local emergency management agencies in dealing with emergencies under the Chemical Stockpile
Emergency Preparedness Program (CSEPP). The main development activity in the emergency management area is the FEMIS.

FEMIS/CSEPP is being developed to open system standards. When complete, it will support any UNIX-based open system hardware set. It is the foundation of the multiple-hazard system. The initial product release took place in December 1994. Follow-on development has been approved and is being funded. FEMA has funded an extension of the FEMIS/CSEPP development effort designed to port v1.1.5 and v1.2 of the FEMIS to their primary platform (IBM RISC). FEMA may implement the FEMIS system nationally once it is completed and may manage it until it is replaced with something better.

Follow-on Laboratory support could take the form of development of individual hazard modules for the system, enhancements to its basic functionality, and configuration management of the in-place system during its lifetime. This effort would extend approximately 12 years into the future.
This effort is currently funded at approximately $\$ 500 \mathrm{~K}$. Follow-on funding for the program by the U.S. Army is expected. Considerable interest in FEMIS has been expressed by nonfederal agencies as well, including state and local emergency managers.

\section{Other Federal Agencies and Nonfederal}

Several other federal agencies fund work at Pacific Northwest National Laboratory. They include the Departments of Agriculture, Commerce, Housing and Urban Development, Interior, State, and Transportation and the National Science Foundation.

The Laboratory's work for nonfederal organizations (i.e., commercial entities) under the 1830 operating contract was \$0.3 in FY 1995 and is expected to be about \$1 in FY 1996. 
Battelle's traditional values serve as a guidepost on the quality journey at the Pacific Northwest National Laboratory. They include the following:

Benefit of humanity-Our operations always must be oriented toward solving significant problems and advancing the quality of life.

Innovation-We aspire to be a world leader in scientific discovery, technical inventiveness, and technological innovation aimed at putting technology to work.

Integrity-We expect ethical behavior on the part of all staff members.

Quality-The hallmark of our activities must be services and products of the highest quality commensurate with the needs and resources of our customers.

Teamwork-We strive to transform creativity and inventiveness into highquality services and products through the teamwork of diversely talented staff members dedicated to achieving a common objective, with strong linkages to the broader community at large.

\section{Corporate citizenship-We must} honor our obligations to society by making Pacific Northwest National Laboratory an economic, intellectual, and social asset to each community in which we operate. And we must aggressively strive to meet all environment, safety, and health goals.

Growth-A world of increasing population, complexity, and interdependence needs a Laboratory that also is growing and advancing in capabilities for serving societal needs.

Earnings-In order to continue and advance, it is necessary that we generate the retained earnings to achieve our objectives, and thus serve our public purposes.

These values come alive through the actions of talented people working together on solving significant problems to maintain the Laboratory's relevance and increase productivity. Management provides the tools and information needed by staff to deliver creative and innovative results to the customer and an environment that is safe, healthy, and personally rewarding. Thus, human resources; information resources; environment, safety, and health compliance; and communication and trust are critical elements for Pacific Northwest National Laboratory to successfully carry out its mission. These elements are discussed in this section of the plan.

\section{Human Resources}

Human Resources is committed to developing and implementing a program that satisfies the needs of the Laboratory while meeting all internal and external requirements. Major elements of Human Resources include general laboratory personnel programs and affirmative action/equal opportunity employment programs.

\section{Laboratory Personnel}

Areas receiving special attention are recruiting and retaining high-quality staff, enhancing staff development, and developing a reward system that supports the Laboratory's strategic goals.

\section{Staff Development}

Laboratory management is moving forward with a formal leadership development effort to enhance leadership performance companywide. The effort builds on core leadership development concepts already in place in the organization and supports the Laboratory's operating principles. The training is designed to give leaders applicable skills for work-related problems and situations. A just-in-time training approach engages management, as specific cues are evidenced in the organization, thus avoiding the niceto-know training and focusing on the need to know. The overall development effort addresses assessed needs including team leadership, feedback and recognition, communication, and change management. This effort is designed to positively influence personal and organizational behavior.

Having a change implementation management process is fundamental to bringing about organizational improvements. Pacific Northwest National Laboratory has designed and is implementing a replicable set of principles and tools to manage the human elements critical to the implementation of strategic business goals. The process systematically identifies personal and organizational barriers to change and generates tactics to increase readiness and decrease the time and resources required for implementation success. The process addresses change at all levels of the organization and is staged and layered to ensure that the principles are provided just in time, and become the basis for a continuous improvement process.

As a part of management processes, we link the Laboratory vision/mission and organizational goals to individual performance objectives through the staff development review process. A major outcome of this linkage is the enabling/empowerment of staff and management to maximize personal and professional growth while maintaining strategic alignment with the purposes of the Laboratory. Thus, the staff development review serves as both the means by which the Laboratory connects its work effort to its goals and objectives and the means for developing individual and organizational capabilities.

The Laboratory has fully automated the Training Information System to streamline training processes including 


\section{Laboratory Staff Composition ${ }^{(a)}$}

\section{(Full- and Part-Time Employees)}

\begin{tabular}{|c|c|c|c|c|c|}
\hline Occupational Codes & Total \# (\%) & PhD & MS/MA & $\mathbf{B S} / \mathbf{B A}$ & Other \\
\hline Managers & 457 (12.2) & 124 & 142 & 136 & 55 \\
\hline Technical Staff & $1703(45.5)$ & 468 & 473 & 553 & 209 \\
\hline Administrative Specialists & $401 \quad(10.7)$ & 9 & 81 & 214 & 97 \\
\hline Technician & $164 \quad(4.4)$ & 0 & 0 & 12 & 152 \\
\hline All Other & $1015(27.1)$ & 0 & 2 & 70 & 943 \\
\hline Totals & 3740 & 601 & 698 & 985 & 1456 \\
\hline
\end{tabular}

(a) Data as of September 30, 1995.

the identification of training requirements, course scheduling and registration, and training documentation. Integral parts of this system are the training catalog and the training requirements questionnaire. The Laboratory's system is linked with the Hanford Site, Washington State University, and Columbia Basin College. This linkage allows direct registration in training activities across organizational lines. This expansion enriches the course offerings available to staff by encompassing the breadth of technical and developmental learning activities offered by each organization.

As part of the staff development process, staff members are encouraged to participate in education programs to enhance their job skills and advance their learning. While many staff members are enrolled in local schools while working full-time, leaves of absence are available for staff electing to attend a university located out of the community. Tuition reimbursements, continuation of benefits, and graduate stipends are available for staff on educational leaves of absence.

Given the critical role of the staff development review process, the Laboratory continually evaluates the effectiveness of the process. We recently completed pilot testing of several staff development review process and training improvements. The improvements focus on promoting the staff development review process as an integral part of operating management and project review, and not an annual event. One outcome of the improvement is that the process will now be in alignment with the business planning cycle.

Additional staff development efforts/ targets include career development workshops, personal and organizational assessments, educational assistance programs, expansion of the training and development curriculum, enhancement of the learning environment, and a training and development approach that promotes "applied learning" by focusing on real-time business requirements and issues.

Human Resources is designing and developing a career planning and development model that will enhance our ability to attract quality staff and develop the current work force. Initial focus will be on scientists and engineers; continuing with technical managers (project, program, and line) and technical specialists. Models will then be developed for the remaining Laboratory population.

\section{Staffing}

Restructuring of the Laboratory's largest client, the Department of Energy, along with reduction in DOE budgets has had a direct impact on the Laboratory's staffing requirements. In recent years the Laboratory's continuing demand for candidates with advanced degrees and senior level staff required a focus on establishing new, innovative methods of identifying highly qualified candidates. Our focus has now shifted to lessening the impact of work force reductions on affected staff and maintaining those human resources unique to our business requirements. The Laboratory's senior managers are working to stabilize our existing programs, seek new programs, and accelerate new business development, all of which will ultimately impact our staffing requirements. Future staffing requirements will consist of key/critical hires and internal transfers and realignments. In order to deliver services in a manner appropriate to reduced staffing requirements, the Staffing Department has been eliminated. Staffing strategies and needs will be addressed by the assigned Human Resource Manager (HRM) for each division/directorate.

Although the need for bachelors, masters, and Ph.D. graduates has been adversely impacted by budget and staff reductions, it remains our intent to continue our ongoing relationships with colleges and universities in order to retain our competitive position for hiring top technical graduates. While on-campus recruiting will lessen during this period, we will continue to use the services of Associated Western Universities, Northwest (AWU-NW) to bring minority and female science and engineering students into the Laboratory for summer and other internship positions. 


\section{Compensation and Benefits}

Pacific Northwest National Laboratory is committed to the design and administration of a total compensation program that encourages and rewards excellence and that attracts, retains, and motivates a highly qualified and competent staff. The Laboratory has . developed multiple job evaluation systems that are periodically reviewed and modified to ensure that all Laboratory positions are defined and valued in a manner that is internally equitable and externally competitive. A new career ladder for our scientists and engineers has been developed to better define their respective levels and to better relate their system to the one used for management and other professionals. In addition, important initiatives are currently being pursued to more closely integrate career management and planning with job ladder design. An annual performance review process, part of the staff development review program, appraises each staff member and helps to ensure that individual goals and contributions are recognized and rewarded.

In the benefits area, a Human Resources goal is to provide cost-effective programs that support the Laboratory's recruitment and retention efforts. In conjunction with Battelle, a key initiative is to provide staff the opportunity of making choices in their benefits program. This program recognizes the diversity of today's work force in offering benefits options and coverage choices. In addition to responding to a competitive labor market, such a program can help manage the cost of future benefits.

\section{Affirmative Action and Equal Employment Opportunity}

The Equal Employment Opportunity/ Affirmative Action (EEO/AA) Office at Pacific Northwest National Laboratory is focused on a results-oriented, active program orientation rather than mere compliance-related activities. Emphasis goes beyond legal and regulatory requirements of the function and aims at instilling the value of diversity into the fabric of the organization. The importance of differing points of view is critical to the creative spirit of the Laboratory. Our activities center around four major areas: special programs, training, compliance, and community outreach. We also want to enhance the Laboratory's ability to attract and retain qualified women and minority staff. Although the focus of our EEO/AA programs is not mere compliance, compliance-related activities can be used to enhance our ability to measure and manage diversity. The following two Equal Opportunity tables provide a breakdown of staff by race and gender.

In response to the Americans with Disabilities Act of 1990 , a process has been defined for placing staff with disabilities who can no longer perform the essential function of their jobs with or without reasonable accommodation. When possible, staff will be reassigned to positions with comparable status and pay. The process requires a thorough review of all vacant positions to determine if the staff member is qualified for reassignment.

By monitoring turnover rates, hire rates, promotion rates, and setting stretch affirmative action goals, we can make changes in our programs to fit the needs of the organization. Our affirmative action goals are set on an annual basis according to an eight-factor analysis computed individually for each of our 26 job groups. This analysis, based in part on the Civilian Labor Force statistics and consistent with equal employment opportunity guidelines, takes into account internal availability, local statistics, and national availability for specific types of occupations. A summary of our availability statistics, which represents the appropriate benchmark for analyzing the composition of our staff, can be found in the Pacific Northwest National Laboratory's FY 1996 Affirmative Action Plan. Since our hiring activity in the future is expected to be limited to critical placements, we expect the majority of our affirmative action efforts to be focused on internal promotions or preparation for future promotion.

As a result of our continued efforts to meet or exceed our affirmative action goals as well as our partnering relationship with Office of Federal Contract Compliance Programs (OFCCP), we were once again successful in achieving a very positive outcome as a result of the 1994 OFCCP audit of our programs.

Our downsizing efforts have been closely monitored to ensure that no adverse impact exists to those in protected classes. Our continued goal during our downsizing efforts is to maintain the positive strides that we have made over the past few years in diversifying our work force. Our retention of minorities and women to date has been very encouraging.

\section{Strategic Planning and Evaluation}

After review of our internal assessments, we have updated our strategic direction and believe it is appropriate to jointly address diversity and qualityof-work-life issues by focusing on creating a supportive work environment because of the following discoveries:

- a supportive work environment is common to both diversity and quality of work life and is a critical factor influencing a person's work experience

- other common issues were identified through these assessments.

This customer feedback indicates a desire for a tailored approach to addressing diversity and quality of work life needs within the divisions and directorates as well as a Lab-wide mechanism to identify and address issues. As a result, we are implementing a three-pronged approach to managing diversity and quality-of-work-life issues:

- creation of a tailored diversity/ quality-of-work-life approach for each division/directorate

- modification of the current Staff Diversity Enhancement Program (SDEP) to become an inclusive Supportive Work Environment User Group

- integration of diversity/quality of work life into our Leadership Program. 


\section{Equal Employment Opportunity(a)}

(1994 Full- and Part-Time Permanent Employees)

\begin{tabular}{|c|c|c|c|c|c|c|c|c|c|c|c|c|c|c|c|c|}
\hline \multirow{2}{*}{$\begin{array}{l}\text { Occupational Codes } \\
\text { Gender }\end{array}$} & \multicolumn{2}{|c|}{ Total } & \multicolumn{2}{|c|}{ Minority Total } & \multicolumn{2}{|c|}{ White } & \multicolumn{2}{|c|}{ Black } & \multicolumn{2}{|c|}{ Hispanic } & \multicolumn{2}{|c|}{$\begin{array}{l}\text { Native } \\
\text { American }\end{array}$} & \multicolumn{2}{|c|}{$\begin{array}{l}\text { Asian/ } \\
\text { Pacific } \\
\text { Islanders }\end{array}$} & \multicolumn{2}{|c|}{$\mid \begin{array}{l}\text { Staff with } \\
\text { Disabilities }\end{array}$} \\
\hline & M & $\mathbf{F}$ & M & $\mathrm{F}$ & M & $\mathbf{F}$ & M & $\mathrm{F}$ & M & $\mathrm{F}$ & $\mathrm{M}$ & $\mathrm{F}$ & M & $F$ & & \\
\hline Managers & 383 & 74 & 22 & 4 & 361 & 70 & 5 & 2 & 4 & 1 & 9 & 0 & 4 & 1 & & 14 \\
\hline Technical Staff & 1362 & 341 & 89 & 30 & 1273 & 11 & 8 & 7 & 21 & 4 & 7 & 3 & 53 & 16 & & 43 \\
\hline $\begin{array}{l}\text { Administrative } \\
\text { Specialists }\end{array}$ & 165 & 236 & 15 & 16 & 150 & 20 & 6 & 4 & 7 & 6 & 0 & 2 & 2 & 4 & I & 7 \\
\hline Technicians & 104 & 60 & 8 & 7 & 96 & 53 & 1 & 0 & 6 & 5 & 1 & 0 & 0 & 2 & & 1 \\
\hline All Others & 321 & 694 & 34 & 72 & 287 & 22 & 7 & 18 & 22 & 40 & 4 & 7 & 1 & 7 & & 22 \\
\hline Totals & 2335 & 1405 & 168 & 129 & 2167 & & 27 & 31 & 60 & 56 & 21 & 12 & 60 & 30 & 1 & 37 \\
\hline \multicolumn{3}{|l|}{ Occupational Codes } & \multicolumn{2}{|c|}{ Minority Total\% } & \multicolumn{2}{|c|}{$\%$ White } & \multicolumn{3}{|c|}{ \%Black } & \multicolumn{2}{|c|}{ \%Hispanic } & \multicolumn{2}{|c|}{$\begin{array}{l}\text { \%Native } \\
\text { American }\end{array}$} & \multicolumn{3}{|c|}{$\begin{array}{c}\text { \%Asian/Pacific } \\
\text { Islanders }\end{array}$} \\
\hline Gender & $\mathbf{M}$ & $\mathrm{F}$ & $\mathbf{M}$ & $\mathrm{F}$ & M & $\mathrm{F}$ & & $\mathbf{M}$ & $\mathrm{F}$ & $\mathbf{M}$ & $\mathrm{F}$ & $\mathrm{M}$ & $\mathrm{F}$ & & M & $\mathrm{F}$ \\
\hline Managers & 83.8 & 16.2 & 4.8 & 0.9 & 79.0 & 15.3 & & 1.1 & 0.4 & 0.9 & 0.2 & 2.0 & 0.0 & & 0.9 & 0.2 \\
\hline Technical Staff & 80.0 & 20.0 & 5.2 & 1.8 & 74.8 & 18.3 & & 0.5 & 0.4 & 1.2 & 0.2 & 0.5 & 0.2 & & 3.1 & 0.9 \\
\hline $\begin{array}{l}\text { Administrative } \\
\text { Specialists }\end{array}$ & 41.1 & 58.9 & 3.7 & 4.0 & 37.4 & 54.9 & & 1.5 & 1.0 & 1.7 & 1.5 & 0.0 & 0.5 & & 0.5 & 1.0 \\
\hline Technicians & 63.4 & 36.6 & 4.9 & 4.3 & 58.5 & 32.3 & & 0.6 & 0.0 & 3.7 & 3.0 & 0.6 & 0.0 & & 0.0 & 1.2 \\
\hline All Others & 31.6 & 68.4 & 3.3 & 7.1 & 28.3 & 61.3 & & 0.7 & 1.8 & 2.2 & 3.9 & 0.4 & 0.7 & & 0.1 & 0.7 \\
\hline
\end{tabular}

(a) Data as of September 30, 1995.

\section{Equal Employment Opportunity(a)}

(1989 Full- and Part-Time Permanent Employees)

\begin{tabular}{|c|c|c|c|c|c|c|c|c|c|c|c|c|c|c|c|c|}
\hline \multirow{2}{*}{$\begin{array}{l}\text { Occupational Codes } \\
\text { Gender }\end{array}$} & \multicolumn{2}{|c|}{ Total } & \multicolumn{2}{|c|}{ Minority Total } & \multicolumn{2}{|c|}{ White } & \multicolumn{2}{|c|}{ Black } & \multicolumn{2}{|c|}{ Hispanic } & \multicolumn{2}{|c|}{$\begin{array}{l}\text { Native } \\
\text { American }\end{array}$} & \multicolumn{2}{|c|}{$\begin{array}{c}\text { Asian/ } \\
\text { Pacific } \\
\text { Islanders }\end{array}$} & \multirow{2}{*}{\multicolumn{2}{|c|}{ | Staff with }} \\
\hline & M & $\mathrm{F}$ & $\mathrm{M}$ & $\mathbf{F}$ & $\mathrm{M}$ & $\mathrm{F}$ & M & $\mathrm{F}$ & M & $\mathrm{F}$ & $\mathrm{M}$ & $\mathbf{F}$ & $\mathbf{M}$ & $\mathrm{F}$ & & \\
\hline Managers & 289 & 33 & 15 & 0 & 274 & 33 & 3 & 0 & 2 & 0 & 7 & 0 & 3 & 0 & $i$ & 9 \\
\hline Technical Staff & 1177 & 228 & 43 & 12 & 1134 & 16 & 3 & 2 & 6 & 1 & 8 & 1 & 26 & 8 & & 39 \\
\hline $\begin{array}{l}\text { Administrative } \\
\text { Specialists }\end{array}$ & 148 & 174 & 18 & 14 & 130 & 60 & 8 & 1 & 7 & 6 & 0 & 2 & 3 & 5 & 1 & 9 \\
\hline Technicians & 162 & 79 & 7 & 3 & 155 & 76 & 1 & 1 & 3 & 2 & 3 & 0 & 0 & 0 & 1 & 6 \\
\hline All Others & 374 & 826 & 37 & 65 & 337 & 61 & 11 & 15 & 21 & 34 & 2 & 5 & 3 & 11 & l & 18 \\
\hline Totals & 2150 & 1340 & 120 & 94 & 2030 & 46 & 26 & 19 & 39 & 43 & 20 & 8 & 35 & 24 & 1 & 81 \\
\hline \multirow{2}{*}{ Occupational Codes } & \multicolumn{2}{|c|}{ Total\% } & \multicolumn{2}{|c|}{ Minority Total\% } & \multicolumn{2}{|c|}{ \%White } & \multicolumn{3}{|c|}{ \%Black } & \multicolumn{2}{|c|}{ \%Hispanic } & \multicolumn{2}{|c|}{$\begin{array}{l}\text { \%Native } \\
\text { American }\end{array}$} & \multicolumn{3}{|c|}{$\begin{array}{l}\text { \% Asian/Pacific } \\
\text { Islanders }\end{array}$} \\
\hline & $M$ & $\mathrm{~F}$ & $M$ & $\mathrm{~F}$ & $M$ & $\mathrm{~F}$ & & $\mathbf{M}$ & $\mathrm{F}$ & $\mathrm{M}$ & $\mathrm{F}$ & $\mathbf{M}$ & $\mathrm{F}$ & & $\mathrm{M}$ & $\mathrm{F}$ \\
\hline Managers & 89.9 & 10.2 & 4.7 & 0.0 & 85.1 & 10.2 & & 0.9 & 0.0 & 0.6 & 0.0 & 2.2 & 0.0 & & 0.9 & 0.0 \\
\hline Technical Staff & 83.8 & 16.2 & 3.1 & 0.9 & 80.7 & 15.4 & I & 0.2 & 0.1 & 0.4 & 0.1 & 0.6 & 0.1 & & 1.9 & 0.6 \\
\hline $\begin{array}{l}\text { Administrative } \\
\text { Specialists }\end{array}$ & 46.0 & 54.0 & 5.6 & 4.3 & 40.4 & 49.7 & 1 & 2.5 & 0.3 & 2.2 & 1.9 & 0.0 & 0.6 & & 0.9 & 1.6 \\
\hline Technicians & 67.2 & 32.8 & 2.9 & 1.2 & 64.3 & 31.5 & & 0.4 & 0.4 & 1.2 & 0.8 & 1.2 & 0.0 & & 0.0 & 0.0 \\
\hline All Others & 31.2 & 68.8 & 3.1 & 5.4 & 28.1 & 63.4 & 1 & 0.9 & 1.3 & 1.8 & 2.8 & 0.2 & 0.4 & & 0.3 & 0.9 \\
\hline
\end{tabular}

(a) Data as of December 31, 1990. 


\section{Work Force Diversity}

An important aspect of our competitive advantage and future viability lies in our ability to create a supportive work environment in which all staff may fully contribute their skills, talents, and experiences to achieving personal and organizational goals. The existence of a supportive work environment is a critical outcome which is supported by the following key principles:

- all staff demonstrate the ability and willingness to value and/or manage differences

- productivity, creativity, and problem solving are optimized through diverse thinking and perspectives

- staff treat each other with mutual respect and dignity

- true equal opportunity exists for all staff

- the work environment is free from harassment

- the work environment allows flexibility

- staff have a clear sense of purpose.

\section{Staff Diversity Enhancement Program}

The purpose of this program is to enhance the Laboratory's ability to attract and retain quality staff members who are minorities and women. The program serves several purposes. It is used as a network for job referrals, professional associations, and community contacts. It also serves as the beginning of a retention program aimed at providing valuable staff members with quality career placement and growth. Seven committees have been focusing on specific needs and issues for each of the seven protected classes (African American, Asian, Hispanic, Native American, Women, Veterans, and Disabled).

The committees have sponsored scholarships, helped with Laboratory placement, proposed Laboratory-wide solutions to issues, joined professional societies (Society for Hispanic Professional Engineers, National Society of Black Engineers, Society of Women
Engineers, the American Indian Science and Engineering Society), and participated in diversity training.

An evaluation of the effectiveness of the SDEP in FY 1995 identified the need to restructure. In the second quarter of 1996, we will expand the membership of the current SDEP to become more representative of the organization. This group will be a supportive work environment team and serve as a pulse of the organization. Lab-wide improvement agenda items will be identified with action plans identified to address needs.

\section{Awareness Training}

To meet the changing needs of the Laboratory, several training programs focus on EEO/AA and diversity. Quarterly, we offer management classes in equal employment opportunity and harassment awareness in the workplace. A shorter version of the course on sexual harassment awareness in the workplace is being given to staff.

Concepts and tools for managing diversity are currently being integrated into the Laboratory's Leadership Development Program efforts. The focus will be to increase managers' effectiveness in creating a supportive work environment where all staff may contribute their skills, talents, and experiences to achieve Laboratory goals. Integration is currently under way.

The Diversity Advisors Program (DAP) was piloted from January to June 1995 . This program represented a reversal in standard mentoring roles. In this program, the Leadership Team member was considered the less-experienced member regarding diversity. Seven members of the Leadership Team were paired with advisors who helped them gain insight/knowledge/awareness into specific diversity issues that they identified. Issues dealt with included race/ gender/support versus research/exempt versus nonexempt. The program received extremely positive feedback from all participants. A common kudos cited by participants was the development of a personal relationship between the pair and the Leadership Team Member's exposure to differences.
Another ten Diversity Advisor pairs will be established during FY 1996.

\section{Other Activities}

In addition to training, special programs, and compliance activities, the Laboratory actively funded and participated in three special initiatives focusing on affirmative action recruiting. These included sponsorship of the American Indians in Sciences and Engineers Society Regional Conference, the Society for Professional Hispanic Engineers, and the Black Engineer of the Year Conference. In FY 1995, the Laboratory also sponsored a workshop at the event, "The Evolution of Diversity: Lessons Learned."

\section{Career Fairs/Job Placement Activities}

Pacific Northwest National Laboratory participates in numerous career fair and job placement activities throughout the year. The level of participation is directly related to the recruitment needs of the Laboratory. Traditional sources for attracting qualified minority and women candidates include

- SER (Hispanic) National Employment Advisory Council

- Hispanic National Achievement Awards Conference

- Society of Women Engineers (SWE) Annual Conference/Job Fair

- GEM Selection Meeting

- National Society for Black Engineers (NSBE) Conference and Career Fair

- Society of Hispanic Professional Engineers (SHPE) Annual Conference

- American Indian Science and Engineering Society (AISES) National Conference

- Black Engineers Awards Conference

- National Organization of Black Chemical Engineers (NOBChE).

We expect our active involvement in NSBE, AISES, SHPE, and SWE to be focused more on a local rather than a national level throughout FY 1996. 
The Laboratory also participates in cooperative work study programs with local high schools and area colleges to help provide women and minority students with requisite skills for employment in industry. The Cooperative Office Education (COE) Program and Inquiry into Science (IIS) Program, provide part-time employment to high school seniors. These jobs may be in any area at the Laboratory and may include entry-level work in the technician and clerical areas.

As shown in the Core Missions section of this Institutional Plan, the Laboratory is actively involved in the educational process from elementary school through postdoctoral studies. Several of these educational programs specifically target women and minorities: Science Alive, Student Research Apprenticeship Program; OPTIONS; HBCU programs; Environment Career Opportunities for Minorities; Life Gets Better; Washington State Mathematics, Engineering and Science Achievement (MESA) Program; and National Consortium for Graduate Degrees for Minorities in Engineering and Science (GEM). Other activities/programs focusing on women and minorities include: Take Our Daughters to Work Day, Washington Science Teachers Association Equal Opportunities in Science Committee, and the Expanding Your Horizons Conferences for Young Women.

\section{Environment, Safety, and Health Management}

Environment, safety, and health (ES\&H) are areas that have always been important at Pacific Northwest National Laboratory. With recent increased emphasis in this area, the Laboratory has augmented its ES\&H program. We have directed our attention toward ensuring that the Laboratory delivers our products in a manner that ensures protection of the environment and the public and ensures the safety and health of workers. The Laboratory is increasing emphasis on disciplined operation of our activities and facilities. The most significant challenges confronting the Laboratory include older facilities that are in need of upgrades and modifications to meet current standards; legacy materials including plutonium, spent nuclear fuel, mixed waste, archived samples, and a few chemicals; implementation of the Nuclear Safety Management Rules (10 CFR 830, 834, and 835); compliance with the recently issued Hanford Facility Permit and other environmental agreements; and integration of Conduct of Operations into the Laboratory's activities and operations, including research.

Perhaps the most difficult challenge is meeting the demand for ES\&H program improvements and pressure to fully comply with requirements in a time of decreasing budgets. DOE is making numerous changes to its set of Rules and Standards that will require evaluation and implementation once issued. These requirements may provide some relief from past practices, but will require additional resources to fully evaluate and to develop appropriate implementation plans and modifications to existing procedures.

The ES\&H program at the Laboratory is described below. Many of our initiatives and ongoing efforts are aimed at the most cost-effective solutions to problems.

\section{ES\&H Goals and Objectives}

The Laboratory's immediate goal is to make substantial improvements in the manner in which we operate our facilities and conduct our work in order to comply with DOE Orders and Directives as well as federal, state, and local regulations. The Operations Improvement Program (OIP) was established in November 1994 to address DOE-RL's concerns with the manner in which the Laboratory was managing its facilities, operations, and activities. The program was established to identify and then address the systemic problems which led to shutdown of a major facility and a plethora of occurrences and audit findings that clearly illustrated a significant lack of disciplined operations and failure to implement requirements at the working level. The aim of the Operations Improvement Program is to implement an organizational structure with clear roles, responsibilities, authorities, and accountabilities that is supported by an efficient management system resulting in sustainable operational excellence. It has been determined to be critical to our future and must be successful if we are to continue to provide research and development for DOE. The program comprises the following four elements:

- Organizational Model: Select and implement an organizational model that has clear roles, responsibilities, authorities, and accountabilities.

- Management System Definition: Identify the appropriate set of standards for the Laboratory's business and establish a management system architecture.

- Implementation: Ensure the implementation of the appropriate standards, the standards-based management system, and the specific outcomes of the eight Operations Improvement Program initiatives. (The initiatives are discussed in more detail later in this section.)

- Program Management: Manage the long-term design and implementation of the Laboratory's operational, organizational structure, and standards-based management system, as well as the near-term development and implementation of the eight initiatives. Ensure integration of the Operations Improvement Program with other Laboratory initiatives.

The Laboratory also has several longterm goals aimed at improving our environment, safety, and health program. These goals include

- completing a graded safety analysis for Laboratory-operated nuclear facilities

- continuing to upgrade and modify facilities to meet current environment, safety, and health requirements through use of general plant projects and line item funding 
- implementing the principles of the Occupational Safety and Health Administration's (OSHA's) Management Guidelines (Voluntary Protection Program) to improve worker safety and health

- minimizing the amount of hazardous waste that must be disposed of by redistributing and reusing materials and by substituting nonhazardous materials where possible

- achieving continuous improvements in environment, safety, and healthrelated areas.

\section{Current Conditions}

Pacific Northwest National Laboratory has allocated additional staff and other resources over the past several years to enhance numerous aspects of our ES\&H program. The reductions of DOE's overall budget will impact the Laboratory (as it will all other sites and contractors). This will inhibit further growth in the ES\&H program and staffing, and is likely to result in decreased resources for this program. The challenge is to make better use of the resources to ensure the health and safety of workers and the public and protection of the environment.

There continues to be escalation of reuirements that we are expected to comply with and implement. Many of these have been coming from within DOE. The recent initiatives on DOE's part to consolidate and reduce requirements in Orders may help relieve some of this, however, there appears to be continued development of DOE Rules and Standards that we will be expected to implement. In today's climate, DOE has an obligation to ensure that new requirements are essential to protecting safety, health, and the environment and that they are cost-effective.

Other regulatory agencies are also developing new requirements and regulations, increasing enforcement of agreements, and expanding the scope of these agreements. This trend, at a time of decreasing budgets, must be considered in light of the real risks to health, safety, and the environment and the benefits associated with implementing the new requirements.

The continuing emphasis on ES\&H by DOE and the Laboratory needs to be clearly focused on improving performance resulting in fewer accidents and incidents, reductions of injuries and illnesses, better control of hazards, and improved compliance with environmental regulations. Portions of the ES\&H program that do not provide benefit in these areas must be eliminated. New requirements that demand increased resources will become increasingly difficult to implement. A risk-based approach must be adopted so that limited resources may be applied to those areas that will result in the greatest benefit. At some point, an optimum investment will be achieved. At that point, further expenditures will result in marginal or no appreciable gain to protection of safety, health, and the environment.

Pacific Northwest National Laboratory endorses the adoption of regulatory standards as they are applied to similar industries in the commercial sector. The focus of the applicable requirements must be the safety and health of both workers and the public and the protection of the environment.

\section{ES\&H Policies, Organization, and Management}

The Laboratory's policy is to provide a safe and healthful working environment and to operate in a manner that ensures protection of the public and the environment. Our policy is to comply with the letter and spirit of all applicable environmental, safety, and health laws, regulations, and DOE Orders.

Two organizational changes have been made that directly affect the ES\&H program and its operation. The first change occurred on November 1, 1994. A new organization was created to operate the Laboratory's three Category 2 Nuclear Facilities. The
Nuclear Facilities organization now includes the Radiological Control Department. The Radiological Control organization was moved to Nuclear Facilities because that is where most of the radiological work of the Laboratory occurs. This places the Radiological Control Department in the organization that is its primary customer and will lead to improvement in the implementation of radiological control in those critical facilities. This organization was recently combined with the Facilities and Operations Directorate to consolidate Laboratory facilities and operations under a single uniform and consistent management system.

The second change involved the establishment of an ES\&H Directorate reporting directly to the Laboratory Director. The functions of the new ES\&H organization are to provide high-quality technical support services to line and facility managers, effective and efficient ES\&H management systems, and limited and focused independent oversight. The new ES\&H organizational structure was completed as planned in June. The elements of this organization include the Environmental Compliance Department, Environmental Projects, Radiological Control Department, Occupational Safety and Health Department, ES\&H Systems Design and Application Department, Independent Oversight Department, and Training and Qualification Department.

Laboratory policy establishes that

- Staff members have a basic responsibility for their own safety. The Laboratory provides a safe and healthful workplace; however, staff members ultimately control their actions in the workplace and, therefore, have responsibility for their own safety and that of their coworkers. They are expected to identify hazards, problems, and concerns and obtain assistance from line managers and ES\&H support services when necessary. 
- Line managers are fully responsible for the safety performance of their staff members. Line management accountability includes recognizing and correcting potential health and environmental hazards of the work conducted, addressing employees' concerns, implementing control procedures and practices to eliminate hazards or reduce them to as low as reasonably achievable, and providing a safe and healthful workplace. Line management is also responsible for implementing job-specific safety requirements and for ensuring the requisite staff training.

- The newly appointed Director of Environment, Safety, and Health has the responsibility and authority to develop the ES\&H program for the Laboratory, provide the technical resources needed to assist line management in its implementation, monitor the implementation of the program, assess its effectiveness, resolve potential conflicts, and provide periodic status reports to the Laboratory Director. This level of management attention underscores the Laboratory's commitment to environmental, safety, and health issues.

The Operations Improvement Program is a major improvement effort for the Laboratory. It is broader than ES\&H; however, the results of the program will have a major impact on our ability to implement an effective and efficient ES\&H program.

The Operations Improvement Program has eight initiatives. Each is described briefly.

- Standards-Based Management System: The objective of this effort is to develop standards that are necessary and sufficient to support the Laboratory's mission and operations that comply with applicable regulatory requirements. This initiative is key to our success. A clear and complete set of standards is necessary to ensure all staff understand expectations and operate to them.

- ES\&H Organization: This effort was aimed at defining and establishing the function and structure of the new ES\&H organization that reports directly to the Laboratory Director.

- Radiological Control: The objective of this effort is to ensure that the Laboratory achieves compliance with and fully implements 10 CFR 835 and the DOE Radiological Control Manual.

- Leadership: This is a key initiative which is critical to our ability to implement standards. It is aimed at improving the environment for supporting the research and development mission and meeting all appropriate ES\&H standards. Without effective leadership, the other initiatives will be difficult if not impossible to implement.

- Audits and Assessments: The objective of this effort is to integrate, focus, and streamline the assessment process at the Laboratory and coordinate external assessments to ensure the greatest benefit to the Laboratory's operations.

- Training and Qualification: This effort will develop an integrated and effective training program, improve training delivery mechanisms, and implement an effective means for record keeping.

- Facility Management: The objective of this effort will be to develop a facility management system and infrastructure that is responsive to our business drivers. The system will apply a risked-based graded approach to the implementation of necessary and appropriate operational controls on a facility basis to meet operational needs of the Laboratory, the assigned organizations, and DOE. This initiative must ensure clear understanding of roles, responsibilities, authorities, and accountabilities for facility operations.

- Conduct of Operations: This initiative will establish management systems that institutionalize the appropriate formality of operations using a risk-based graded approach. The program will ultimately develop clear and precise standards for defining graded approach strategies, performance based on graded approach, and methods to monitor successful program implementation.

An estimate of the overall cost for the ES\&H program is provided in the table. This information is derived from the ES\&H Management Plan. There are approximately 170 staff dedicated full-time to the various aspects of the ES\&H program. This is about 4 percent of the total Pacific Northwest National Laboratory staff. Future growth is not anticipated to be as great as during the past few years. At some point, an optimum investment must be achieved, and at that point, further investments will result in only marginal improvements.

\section{ES\&H Plans and Initiatives}

The Laboratory continues to work on improving and enhancing environment, safety, and health program elements. The ES\&H Management Plan contains a more complete description of the Laboratory's program. There are many efforts at the Laboratory currently ongoing within the program that are designed to control and reduce our risk and ensure the protection of workers, the public, and the environment. The specific program elements discussed below were chosen to represent areas where progress is being made.

\section{Occupational Safety and Health}

- During this past year, Pacific Northwest National Laboratory developed and implemented the concept of "Health and Safety Teams" composed of one industrial hygienist and one occupational safety engineer. These teams were assigned to each line organization and the members are expected to spend the majority of their time with those organizations working to integrate health and safety requirements and provisions into the daily work routine. These teams are designed to provide the assistance needed by the line organizations to properly implement worker protection requirements.

- The Laboratory continues to work on ergonomic issues by providing workstation analysis and 
training as part of the industrial hygiene program. Since instituting our education program on carpal tunnel syndrome 2 years ago, we have had increasing reports of problems and numerous requests for evaluations. At this time, requests for assistance exceed our available resources.

- The Laboratory is following the schedule established in our implementation plan submitted in FY 1994 for implementing DOE 5480.7A.

- We are specifically targeting a few organizations that have historically high rates of injuries involving backs, hands, and fingers; strains and sprains; etc. The two programs being tested are a "Back Injury Prevention Program" and a "Behavior-Based Safety" approach called BSAFE. Both call for significant staff involvement. The latter, BSAFE, has been developed and is operated by the staff members with some guidance from a safety professional. If either or both of these pilots are successful, they should be expanded to other parts of the Laboratory.

- Industrial hygiene developed a process to clearly identify and document hazards to which workers may be exposed to be used by physicians for determining appropriate targeted medical exams.

- The lock out and tag out program has recently been totally revised to ensure that it complies not only with the present OSHA standard, but also DOE 5480.19. The new procedure is not yet being implemented due to facility management issues that must be corrected by efforts of the Operations Improvement Program and due to the lack of resources to fully implement the work planning documentation requirement and the verification activities associated with the new standard. The most significant safety related (rather than documentation related) changes to the lock and tag program are being implemented through an interim change notice until the correct organizational configuration and resources are made

ES\&H Plan Funding Request ${ }^{(a)}$

( $\$$ in Millions)

\begin{tabular}{llllll} 
& \multicolumn{5}{c}{ Fiscal Year } \\
& $\mathbf{1 9 9 3}$ & $\mathbf{1 9 9 4}$ & $\mathbf{1 9 9 5}$ & $\mathbf{1 9 9 6}$ & $\mathbf{1 9 9 7}$ \\
\hline Expense & 0.0 & 0.0 & 0.7 & 4.3 & 4.0 \\
Capital Equipment & 0.05 & 0.09 & 0.07 & 0.42 & 0.44 \\
Capital Construction & 5.3 & 4.7 & 4.0 & 3.8 & 3.8
\end{tabular}

(a) Includes all items covered in the ES\&H Management Plan.

available for full implementation. There is no increased hazard to workers from the current situation.

- The Corrective Action Tracking System (CATS) developed during 1994 to track and manage audit/ assessment findings and subsequent corrective actions is in the process of being implemented. It was designed to centralize the coordination effort of assessment, condition, and action data and automate the transfer of information among participants to provide the most effective, efficient means to accomplish the tasks involved.

- Voluntary Protection Program (VPP): Most firms that are part of the VPP are production oriented and very different from the research and development operations performed at the Laboratory. We are planning an effort to identify and then benchmark companies similar to the Laboratory that are currently participating in OSHA's VPP. We will review their approach to meeting the OSHA Safety and Health Management Guidelines and apply lessons learned to our approach for application for VPP status.

\section{Radiological Control}

- The Laboratory prepared an implementation plan to meet the requirements of $10 \mathrm{CFR} 835$ and has begun working toward full implementation which was required by January 1 , 1996. The procedures to implement the requirements of $10 \mathrm{CFR} 835$ were developed during FY 1995. These procedures will be available for implementation as they are prepared. This same approach will be used to develop the further requirements of the DOE Radiological Control Manual. Verification that these procedures have been implemented is essential to our ability to demonstrate compliance with 10 CFR 835.

- We did not fully implement the DOE Radiological Control Manual due to failure of line management to completely implement provisions of the manual and failure of the radiological control organization to adequately enforce the manual. A revised implementation plan for the RadCon Manual has been submitted to DOE. Implementing procedures must be prepared for each of the Articles that are not covered by 10 CFR 835 .

\section{Nuclear Safety}

- Pacific Northwest National Laboratory currently operates three nuclear facilities. The Laboratory also has actively worked to reduce inventories in our facilities to lower the category or eliminate the facility from the list of nuclear facilities entirely. During the past year, one facility has been removed from the list of nuclear facilities. A substantially increased level of effort will be required during the next several years to reach full compliance with the Nuclear Safety 
Rules. Implementation plans to prepare and revise safety analyses and technical safety requirements will be prepared and submitted to DOE as required.

- An intensive effort has been under way to revise the safety analysis for these facilities. Neither the funding level nor the time allotted will be sufficient to complete the three Safety Analysis Reports (SARs) that are required for the Laboratory's operating nuclear facilities. Due to budget constraints, EM will not be able to provide funding for this effort beyond 1995. The Laboratory is operating to currently approved SARs and OSRs and will continue to do so until the revised SARs are approved by DOE.

\section{Environmental Protection}

- Pacific Northwest National Laboratory is working with other Hanford Site contractors and DOE-RL to prepare an air operating permit that will cover Laboratory facilities onsite. This permit is required to comply with Washington State regulations (WAC 173-460) that stem from the Clean Air Act. There will be additional resources needed to meet the provisions of the permit. The current activities are placing a great burden on the Environmental Compliance Section due to constrained resources. Following submission of the permit application now in preparation, detailed negotiations must be conducted with the regulators. These discussions should result in the State issuing a final permit which will then require action on the Laboratory's part to implement and maintain compliance with indefinitely.

- The Laboratory's Waste Minimization and Pollution Prevention Awareness Plan consists of a Laboratory-level plan and specific pollution prevention plans for ensuring ongoing waste minimization activities in the operations organizations. Pacific Northwest National Laboratory intends to strengthen the system for reporting waste minimization progress throughout the Laboratory. The Laboratory is incorporating advice and information from the waste generator's perspective into the overall administration of the pollution prevention program to more accurately document the extent of pollution prevention activities throughout the Laboratory, to identify areas of potentially significant waste reduction, and to apply pollution prevention techniques. We have received funding for enhancement of the pollution prevention program through the DOE-EM Five-Year Plan and from Energy Research.

- Under the terms of the work plan submitted to Washington Department of Ecology under Consent Order DE 91NM-177, several permit applications for liquid effluents are required to be submitted by the Hanford Site over the next 5 years. These permits will require action to implement and maintain compliance.

- The Washington Department of Ecology issued the final Hanford Facility Permit in the fall of 1994. This permit provided the Laboratory with its first Part B RCRA Permit for its Waste Storage Facility (305-B) and includes numerous other requirements for management of RCRA waste on the Hanford Site with which the Laboratory must comply. We are in the process of reviewing several other Part A permits with the intent of withdrawing or closing several locations that have not and will not treat waste. Negotiations have begun with WDOE on this matter.

- Legacy waste issues: Activities in this area have traditionally been funded by DOE-EM. There are, however, emerging issues that are likely to require additional resources which are becoming more difficult to acquire through that funding source. It is anticipated that resources will be necessary to resolve both current and future (i.e., potential) legacy waste issues. Sources of this potential waste include archived samples, material reserved for future use, and material left by departing or retiring staff members.

- The Laboratory continues to focus substantial resources on meeting DOE National Environmental Policy Act (NEPA) implementation requirements. DOE requirements issued during FY 1992 in a letter from DOE-EH include a significant increase in the number of Information Bulletins that must be prepared for categorically excluded activities. Although there has been some modification to these requirements, there is a continued need to review activities for NEPA compliance and to verify that the activities fall within specific Categorical Exclusions. The 1994 Secretarial Policy on NEPA was primarily aimed at environmental assessments and environmental impact statements. We expect some continued streamlining during the coming year.

\section{Energy Research Funded ES\&H Improvement Items}

In FY 1995, Energy Research began to provide operating funds for some ES\&H initiatives. The funding is available for one-time costs or start-up costs associated with ongoing activities, but is not available to support continuing ES\&H program elements.

The following initiatives were funded during FY 1995:

- Safety and Health Information Management System (Phase I) provides rapid accident reporting and investigations. It also automatically generates the reports needed by DOE, the regulatory agency, and Laboratory management. It is capable of providing rapid trend analysis that is important to identifying and then addressing emerging injury or illness problems. 
- The Chemical Management System is a central, computerized chemical inventory that was established for the Laboratory several years ago to enhance management's efforts to ensure environment, safety, and health compliance. The system is being enhanced to provide access to the Hanford Site MSDS system and provide for waste disposal requests.

- Computer-Based Training is an effective and efficient means of providing just-in-time training to staff who need it, when it is needed, and where it is needed. The efforts during 1995 allowed us to expand this effort to keep personnel current on training and provide uniform information in a highly efficient manner. This capability is essential to the efficient operation of a user facility such as the EMSL.

- Pollution Prevention is aimed at conducting "Opportunity Assessments" to identify waste streams for efforts to reduce hazardous waste generation. The goal of the pollution prevention program is minimizing or preventing waste at the generating source thereby decreasing or eliminating the amount of waste requiring disposal.

- Multiprogram Energy LaboratoriesFacilities Support (MEL-FS) ES\&H projects are aimed at correcting selected deficiencies identified during past audits and inspections for which sufficient overhead funds have not been identified.

- Removal of chlorofluorocarbons from the heating, ventilating, and air conditioning involves inventorying building system equipment that uses refrigerants to classify the equipment and list the refrigerant type and quantity. A database will be created to maintain the inventory.

The following short-term initiatives are the candidates for DOE-ER funding in FY 1996:

- Ergonomic program enhancements: Injuries and illnesses associated with carpal tunnel syndrome amount to 32 percent of Pacific Northwest National Laboratory's lost workday cases and nearly 50 percent of our total lost workdays. This program will substantially increase the safety and health of our work force, and result in a significant reduction in our injury and illness rates.

- Verification of 10 CFR 835 implementation: Once the procedures have been prepared and issued for implementation, some level of verification must take place to ensure that implementation has occurred. Without this, we cannot be ensured of actual compliance with requirements. During the development of new procedures and the implementation steps taken, every effort will be made to ensure that there is complete implementation.

- Safety and Health Information Management System Phase II: Phase II will incorporate important industrial hygiene information such as exposure monitoring data and medical information to further document workplace hazards and exposures. This tool will provide an efficient mechanism to report and analyze important safety and health information and present it in a usable way to both safety professionals and line managers.

- Expansion of the Behavior-Based Safety Program: The current pilot program appears to be returning positive results after only 2 months of operation. Since very positive experiences have resulted at other locations that have applied this program, we anticipate that this pilot will demonstrate positive results and we will need to apply it to other areas. This program will result in increased safety and health of the work force, and will reduce our injury and illness rates.

Energy Research also provides funding for construction, capital equipment, and facility upgrades and improvements. Following is the list of current activities which Pacific Northwest National Laboratory is undertaking with this funding:

- General Plant Projects (GPP): Composed primarily of discrete corrective activities required to bring DOE-ER facilities into compliance with ES\&H regulatory requirements.
- General Purpose Facilities (GPF): FY 1993 Life Safety Code Compliance provides immediate correction of code noncompliance in four multiprogram laboratory and support buildings. FY 1995 Line Item, Electrical Safety Rehabilitation will provide replacement of deteriorated wiring and outmoded equipment in multiprogram buildings.

- Capital Equipment and General Purpose Equipment (GPE): Funding for FY 1995 has been authorized for refrigerant replacement equipment. No general purpose equipment funding is included.

The Laboratory will continue to give a high priority to funding environmental, safety, and health activities; however, additional requirements will generally result in higher costs. Pacific Northwest National Laboratory must receive direct funding for implementation of additional requirements.

\section{Environmental Restoration and Waste Management Activities}

The DOE and the Laboratory are committed to achieving compliance with applicable laws, regulations, and federal facility agreements aimed at protecting human health and the environment. Resources are being focused to 1 ) assess and clean up inactive waste sites and facilities, 2) continue safe and effective waste management operations while emphasizing the systematic minimization of waste materials generated (see the Waste Minimization Plan Funding table), and 3) coordinate a focused research and technology development program for application to waste management and environmental protection. DOE-EM is consolidating environmental cleanup, compliance, and waste management activities identified in the Five-Year Plan.

There are numerous activities of vital importance to ES\&H at the Laboratory that are currently funded by DOE-EM. Some of these activities include the following: waste management operations, RCRA environmental compliance, NEPA compliance, 324 B-Cell 


\title{
Waste Minimization Plan Funding
}

\author{
( $\$$ in Millions)
}

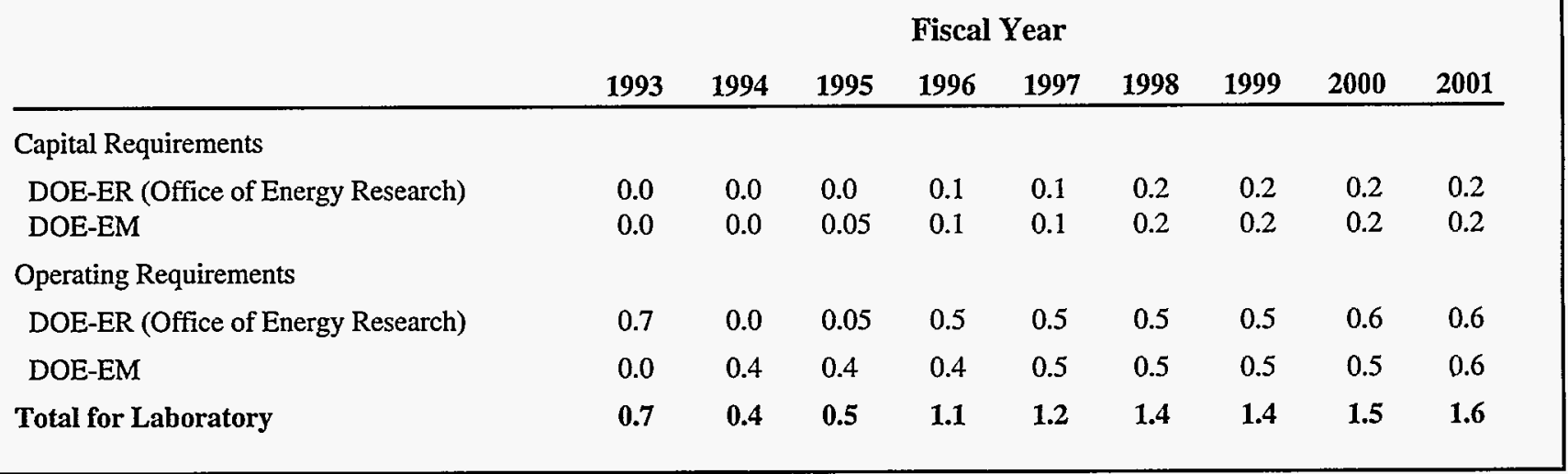

\section{DOE-EM ES\&H Funding Requests}

\section{(\$ in Millions)}

\begin{tabular}{|c|c|c|c|c|c|c|c|c|c|}
\hline & \multicolumn{4}{|c|}{ Authorization } & \multicolumn{5}{|c|}{ Planning Case } \\
\hline & 1993 & 1994 & 1995 & 1996 & 1997 & 1998 & 1999 & 2000 & 2001 \\
\hline EM-30 Expense Totals & 27.4 & 34.7 & 43.4 & 56 & 57.2 & 48.2 & 48.9 & 41.9 & 38.9 \\
\hline EM-30 Capital Totals & 1.9 & 3.5 & 1.1 & 2.7 & 2.4 & 2.6 & 2.2 & 2.3 & 0.5 \\
\hline
\end{tabular}

Cleanout, Tank Integrity Inspections, surveillance and maintenance activities at old facilities, effluent monitoring, and building improvement and renovation projects to bring old facilities into compliance with current codes (e.g., National Fire Protection Association and the National Electrical Code).

The Resource Projections section of this Institutional Plan identifies all the Laboratory activities requested from DOE-EM, including those shown in the Five-Year Plan. The following funding highlights are included in the Environmental Restoration and Waste Management Five-Year Plan funded by DOE-EM:

- General Purpose Facilities: Comprises major facility compliance/ renovation upgrades to strategically important DOE laboratories in the 329 Building.
- General Plant Projects: Comprises discrete corrective activities required to bring DOE-EM facilities into compliance with environment, safety, and health regulatory requirements. Typical projects include life safety upgrades (including electrical switchgear and distribution systems repairs and/or replacements and/or upgrades), high-efficiency particulate air (HEPA) filter housing upgrades, sampling systems installations, and fire protection upgrades.

- General Purpose Equipment: Includes waste minimization program equipment and storage tank upgrades for radioactive mixed waste.

- Operating: Includes NEPA compliance program, effluent monitoring, waste minimization and pollution prevention, essential drawings, liquid waste source control, waste management operations, laboratory surveillance and maintenance, and cleanup of production support laboratories assigned to Pacific Northwest National Laboratory.

Specific facility upgrades are discussed further in the Site and Facilities section of this plan.

\section{Hanford Site-Wide Funded ES\&H}

The Hanford Site Landlord Program (EM-60) enhances the Hanford Site general purpose infrastructure in support of the Hanford Site cleanup. The general purpose infrastructure consists of buildings, systems, and equipment that, by design or use, are not essentially dedicated to a single-program mission. The Landlord Program primarily consists of capital funding (see 


\begin{tabular}{|c|c|c|c|c|c|c|c|c|c|}
\hline \multicolumn{10}{|c|}{$\begin{array}{l}\text { DOE-EM Landlord ES\&H Funding Requests } \\
\qquad \text { (\$ in Millions) }\end{array}$} \\
\hline & \multicolumn{4}{|c|}{ Authorization } & \multicolumn{5}{|c|}{ Planning Case } \\
\hline & 1993 & 1994 & 1995 & 1996 & 1997 & 1998 & 1999 & 2000 & 2001 \\
\hline EM-60 Capital Totals & 5.1 & 3.5 & 5.0 & 14.6 & 13.7 & 19.5 & 13.3 & 14.1 & 4.7 \\
\hline EM-60 Expense Totals & 0.4 & 0.8 & 0.3 & 0.9 & 2.0 & 1.1 & 0.4 & 0.2 & 0.2 \\
\hline
\end{tabular}

\begin{tabular}{|c|c|c|c|c|c|c|c|c|c|}
\hline & \multicolumn{9}{|c|}{$\begin{array}{c}\text { ES\&H Management Plan } \\
\text { ( } \$ \text { in Millions) }\end{array}$} \\
\hline & \multicolumn{4}{|c|}{ Authorization } & \multicolumn{5}{|c|}{ Planning Case } \\
\hline & 1993 & 1994 & 1995 & 1996 & 1997 & 1998 & 1999 & 2000 & 2001 \\
\hline Core Program & 15.3 & 16.0 & 17.4 & 19.4 & 20.1 & 20.6 & 21.1 & 21.8 & 22.3 \\
\hline Compliance Program & 0.55 & 1.1 & 1.0 & 9.8 & 9.9 & 5.2 & 4.4 & 3.6 & 3.7 \\
\hline Improvement Items & 0.0 & 0.0 & 0.06 & 1.6 & 0.7 & 0.3 & 0.3 & 0.3 & 0.3 \\
\hline Total for Laboratory & 15.85 & 17.1 & 18.5 & 30.8 & 30.7 & 26.1 & 25.8 & 25.7 & 26.3 \\
\hline
\end{tabular}

the Landlord Funded ES\&H table) for ongoing replacements and enhancements to general purpose facilities: water service; electrical distribution; roads; steam; building additions or replacements (if not used by a single program); and other general environment, safety, and health equipment.

General purpose facilities projects are 325 and 324 Buildings Facility Compliance, Whole-Body Counter Facility, and Building Utility Replacement.

General plant projects activities include fire protection upgrades, 325 Building system improvements, and hot cell refurbishments.

Operating-expense funded activities include National Electrical Code compliance activities and preparation of Safety Analysis Reports for Laboratory-operated nuclear facilities.
Significant Laboratory resources are devoted to environment, safety, and health activities. The requirements are so numerous and costly, however, that the overhead funding cannot bear the entire burden of these added requirements. Laboratory organizations are aggressively seeking direct funding via proposals to the appropriate Program Office for requirements that are beyond the reach of the overhead budgets. The effects of failing to obtain direct funding for these activities may be a limited or delayed remediation of deficiencies; the levels of surveys, reviews, and revisions of procedures would have to be substantially reduced; and the Safety Analysis Report upgrade program could be negatively impacted.

\section{Information Resource Management \\ Information Technology Vision}

Pacific Northwest National Laboratory's Information Technology (IT) vision is to be a Laboratory without walls - where staff work interactively with each other and in collaboration with universities, government, industry, and the international community to solve significant national problems. Our greatest challenge is to develop knowledge, firmly based on the access to and use of key data and information. 
Therefore, we must continue to invest in information technology to enhance effectiveness in our missions of energy, environment, economic competitiveness, education, and national security; facilitate the advancement and transfer of science and technology; increase management and administrative efficiency; and deliver quality products to our customers.

We envision four key Information Technology strategies that support our vision: 1) empowering the user, 2) defining and meeting the information technology needs of the Laboratory, 3) making effective use of information technology, and 4) ensuring cost-effectiveness.

\section{Empowering the User}

Users will achieve scientific, engineering, and technical excellence by having

- convenient access to required information, regardless of the physical location of the resources, information, or the user

- tools, training, and expertise to manipulate information appropriately

- the ability to choose the work style best suited to the task, rather than be constrained by tools and methods

- simple methods to communicate and collaborate with other individuals, work groups, external businesses, and institutions.

\section{Defining and Meeting Informa- tion Management Needs}

Pacific Northwest National Laboratory's business strategies will drive its information strategies. Laboratory managers and staff have the primary responsibility to translate their requirements into plans and actions for managing information as an important and strategic asset. To this end, information systems requirements will be

- clearly and concisely stated

- consistent with established standards for development, design, implementation, operation, and maintenance
- consistent with regulatory requirements and the needs of Laboratory customers.

\section{Effective Use of Information Technology}

Pacific Northwest National Laboratory will manage Information Technology as a strategic resource while continuously evaluating technology advances seeking improved capability to meet our mission objectives. We will maintain an effective Integrated Computing Environment (ICE) infrastructure that provides

- utility, where the underlying technology is invisible to the user

- standard services to enable local, national, and international communication and collaboration

- standards and guidelines, facilitating information interchange

- electronic methods for accomplishing routine business functions, reducing the use of paper

- uncompromised production reliability and stability, while allowing the evaluation and exploration of advanced technology.

\section{Cost-Effectiveness}

We will establish internal processes which enhance the efficiency of the computing environment to

- assign the true costs for computer systems and services to those receiving the benefit of those systems and services

- assist users in managing resources to ensure that acquisitions and upgrades are conducted in a disciplined manner and in a manner that enhances the Laboratory's capabilities

- continuously evaluate internal services and benchmark levels of support and services with other laboratories, universities, and the private sector.

\section{Environment and Accomplishments}

Information Technology continues to play a significant role in support of the Laboratory's research, development, technology transfer, and education missions both as the Hanford Site research and development contractor and as a DOE-ER multiprogram laboratory. While the Laboratory is making significant progress in developing an effective and efficient computing and telecommunications infrastructure, much remains to be done. Major computing and information technology resource investments include

- local and wide-area network infrastructure expansion and upgrades

- scientific and engineering computing infrastructure and applications with emphasis on high-performance computing, modeling, simulation, and user collaboration

- administrative and management information systems with emphasis on enterprise-wide applications, open systems, seamless integration, and distributed client-server computing with a centralized repository

- multiprogram, multilaboratory collaboration and leadership as well as outreach to the academic and industrial communities.

\section{Infrastructure}

Pacific Northwest National Laboratory's Integrated Computing Environment provides the computing, network, information, and service resources that support scientific and engineering research, development, and technology deployment; management of the business; and access to scientific and technical information. The Integrated Computing Environment consists of a local and wide-area network infrastructure, the attached computing resources from office workstations to supercomputers, software tools, utilities and applications, and services to support the user community. 
The Integrated Computing Environment supports file, print, communications, information, and access services for workstation, midrange, mainframe, and supercomputing systems and provides a full range of input/output and support services for the entire Information Technology life cycle.

Over 8,000 office, laboratory, and portable workstations are in use. Ranging from IBM PCs and compatibles to Macintosh and RISC architecture UNIX systems, workstations are used for software development, science, engineering, and business computing, word processing, desktop publishing, spreadsheets, databases, electronic mail, project management, personal scheduling, multimedia applications, data acquisition and control of laboratory instruments, field use, as well as providing access to people, information, and systems across local and national networks. Approximately half of these systems are located on the desktop with the others supporting the infrastructure (e.g., servers) or are dedicated to other activities (e.g., data acquisition, control, training, etc.).

Voice communication and messaging are provided by an InteCom IBX S/80 digital voice/data telephone system serving the 45 percent of Laboratory staff located in private facilities. The Hanford Site Integrated Voice and Data Telephone System serves the remaining 55 percent of Laboratory staff located in DOE facilities.

Centralized video conferencing is supported by a two-switched $56 \mathrm{k}$ services located in conference rooms. Similar systems are located at Battelle's corporate and the Laboratory's Washington, D.C., offices providing an alternative to travel for meetings and collaboration. Mbone functionality is supported by the Laboratory network and is primarily used in support of conference or collaborative activity, but is not yet a universal service to all desktops.

PNLnet is based on multiple FDDI backbones with 120 attached Ethernet segments. There are 6,523 workstation connections, 481 network printers,
123 servers, and 30 routers. Fifty servers provide generalized services while another 73 support application-specific services. The network serves 116 buildings with over 20 miles of fiber in the primary FDDI ring and another 50 miles of fiber interconnecting Laboratory buildings. In FY 1993, the network was converted to TCP/IP replacing several proprietary protocols. The Laboratory is connected to the Energy Sciences NETwork (ESNET) by an FTS-2000 T1 circuit to Livermore, which is scheduled to be increased to T3 according to the current DOE-ER upgrade plan. The link also supports DOE-EM traffic from other Hanford contractors in accord with a Memorandum of Understanding between DOE-ER and DOE-EM. We initiated network-based, unattended, backup and restore for user workstations and automatic password expiration.

$\mathrm{T} 1$ service is also available between Pacific Northwest National Laboratory's Richland and Seattle, Washington, campuses for access to the National Science Foundation's regional research and education network, NorthWestNet. This provides Internet access backup for the ESNET link and direct access to the Laboratory from the regional and national universities as well as industry and the international community via Internet.

High performance graphics workstations (e.g., Silicon Graphics, Intergraph, and others) give access to advanced tools for still-frame and full-motion three-dimension color visualization. A multimedia laboratory provides facilities for high quality multimedia productions and supports graphics laser printing, audio and video recording, as well as CD-ROM and videotape production.

Midrange systems are being replaced with individual and/or clustered high performance workstations for both scientific and administrative computing. Four clusters are operating (DEC, HP, IBM, and Sun). A limited VAX capability serves as the Internet mail gateway and supports a declining number of legacy applications requiring VAX compatibility.
Supercomputing requirements are satisfied by a combination of in-house systems, remote access to other DOE sites (Los Alamos, Argonne, and NERSC), and the Laboratory's participation in the Concurrent SuperComputing Consortium with access to the Intel Delta at Cal-Tech. In-house resources include a Kendall Square Research KSR2 system with 80 processors, 2.56 GBytes of main memory, and 40 GBytes of disk storage installed in the interim EMSL, a small Intel iPSC 860 system; and the several clusters mentioned earlier.

The Business Information Systems infrastructure is distributed across three environments. A large-scale, IBM-compatible system operated by the Hanford operations and maintenance contractor is used for off-primeshift batch processing. A midrange Sequent S81 parallel processor running Oracle under UNIX and operated by Pacific Northwest National Laboratory is used for on-line management and project reporting. Network servers are used for transaction processing and user access to information distributed from the Sequent using Sybase client-server relational database technology and graphical front end tools (e.g., Business Objects, OMNIS 7.0).

An Electronic Forms, Routing, and Authorization (EFRA) infrastructure was completed in FY 1994. The software and controls were subjected to a comprehensive external review process. This infrastructure is currently being reviewed by $D O E$ for acceptability as the primary means for authorizing procurements and for entering and approving time distribution using an electronic system.

Scientific and Engineering Computing Scientific applications are developed and/or used in most major disciplines with key activities in computational chemistry modeling and simulation; environmental contaminant characterization, transport, and remediation models (atmospheric, oceanic, surface water, and subsurface); global climate and coupled atmospheric/ocean models; 
environmental database development; and thermal hydraulics fluid-flow modeling and simulation.

The most important efforts in computational chemistry in FY 1994 were the redevelopment and/or adaption of important production codes to parallel processing on the KSR2. This included the determination of the behavior of molecular processes at the watermineral interface, prediction of pollutant migration in surface and subsurface regimes, characterization and redesign of biodegradive enzymes for cleanup, development of graphical user interfaces, and graphical visualization applications emphasizing the analysis of mineral systems.

Pacific Northwest National Laboratory has developed a number of Hanford and DOE-wide information systems for scientific data. These include the Tank Waste Information Network System (TWINS), a multimedia capable system, which links waste tank information from Rocky Flats, Oak Ridge, Savannah River, Idaho, and Hanford into a common systems hub located at the Laboratory with access provided to a wide range of external customers across the DOE complex; Hanford Environmental Information System (HEIS) which stores, manages, and aids in the analysis of Hanford's environmental sampling data that are crucial to Hanford's environmental cleanup and restoration mission. Major HEIS components have been operational for over 2 years and records are being added at the rate of about 350,000 chemical and physical analysis records per year with access provided to staff from Hanford, the Washington State Department of Ecology, and the Environmental Protection Agency.

Other systems development includes real time data acquisition and process control, artificial intelligence, satellite image processing, computational fluid flow, molecular modeling, general purpose graphics, scientific database systems, computational chemistry, etc.
Example developments in FY 1994 include

- advanced thermal-hydraulics code development and NRC qualification for thermal response of dry-storage of nuclear fuels

- atmospheric models for contaminant transport and dispersion, chemical and physical transformation, and deposition to determine the continental and oceanic fate of air pollutants

- experimental data systems and measurement technologies to improve the performance of atmospheric General Circulation Models

- National Radiobiology Archives information system for DOE-wide use which contains a dose-andeffects database summarizing individual animals, an inventory of National Radiobiology Archives holdings, and a document and specimen archive

- refinement of models which simulate the movement of water, contaminants, and sediment in oceans, estuaries, and rivers

- thermal-hydraulic safety code development for the Hanford Tank Safety Mitigation and Flammable Gas Projects

- special purpose data acquisition, database, and a three-dimensional graphics system for the analysis of energy use in homes, apartments, and commercial buildings including software development for analyzing the energy efficiency of buildings during the design phase

- high-resolution, three-dimensional, subsurface modeling tools for application on massively parallel computers supporting the design and evaluation of in situ waste treatment technologies at the Hanford Site

- computer modeling of microbial enzymes making a major contribution to understanding the basis for rational enzyme redesign using genetic engineering with potential for environmental cleanup applications
- the on-line library electronic access system consisting of several complementary systems was expanded with addition services and the systems were consolidated on a single platform in FY 1994.

Pacific Northwest National Laboratory's optical digital recording system was a finalist for the prestigious Smithsonian Award in the category of science. Laboratory staff received a Federal Laboratory Consortium award for the development of fast, adaptive communications software for parallel supercomputers, and received a plaque of appreciation from DOE-EH for the selection of the Laboratory's Chemicals Management System (CMS) for transfer to Brookhaven National Laboratory under the Worker Protection Pilot Program.

Business Information Systems Information systems development is based on an annual budgeting process consistent with the enterprise-level strategies developed as a part of the WISDM whole systems model developed in FY 1990. Systems development is based on the principle that information is an organizational asset and is collected, organized, protected, and made conveniently available to authorized staff. Applications interfaces are designed to have a common look and feel based on generic user interfaces and operating procedures. Requirements are developed up front by a group representing all stakeholders. Data is entered only once at its point of origin and made electronically available for re-use in multiple applications.

New systems are implemented using network-based client-server technology. SQL server is the preferred technology for our applications and front-end tools provide the user interface and database access. Application and utility software are acquired or developed to support all three mainstream Laboratory platforms; PC and compatibles, Macintosh, and UNIX. Accomplishments in the development of business information systems in FY 1994 included

- Information Distribution Manager (IDM), a network server-based 
system and client tools for electronic distribution of business information, was moved into production status. Information Distribution Manager provides a common user interface for more timely and effective access to legacy information (on-line, downloadable, with custom user views and easy reporting) and reduces the demand for paper and fiche reporting.

- A system was implemented to support the use of purchase cards for the acquisition of items valued less than $\$ 2500$ avoiding the cost of internal purchasing overheads.

- Completed development of electronic purchase requisition entry system which front-ends an electronic purchasing system providing electronic entry, routing, and authorization of purchases. The system was rolled out in FY 1994, but implementation of the electronic signature module is still pending DOE approval.

- Completed implementation of the database core and modules for compensation, recruiting, and work force diversity on the Human Resources System using PeopleSoft commercial software.

- Completed a major blockpoint upgrade and initiated a front-end to CATS, the Safety Health Information System, which supports findings and tracking of safety-related deficiencies.

- Implemented a network bulletin board system, PNLInfoBrowser tool (Internet compatible browser), and a World-Wide-Web server, and piloted the publication of Laboratory administrative manuals in electronic form.

- Implemented a recycling program for software with Greendisk of Woodinville, Washington. Excess software media and manuals are shipped to Greendisk for recycling. Media from over 8000 software packages have been recycled in FY 1993-1994 reducing cost and eliminating landfill use for software media and manuals.

Collaboration and Outreach Within the DOE's Energy Research program,
Pacific Northwest National Laboratory staff continue to provide interlaboratory coordination, program direction, and leadership for the Atmospheric Studies in Complex Terrain (ASCOT), Atmospheric Radiation Measurement (ARM), and Computer Hardware, Advanced Mathematics and Model Physics (CHAMMP) programs.

Continued Laboratory staff collaborations with Argonne National Laboratory and five industrial firms, Allied Signal, Amoco, DuPont, Exxon, and Phillips, developing scalable algorithms on the Laboratory's Kendall Square KSR2 and Argonne's IBM SP1 parallel systems for grand challenge applications in computational chemistry.

Laboratory staff participation on standards committees include ANSI X3T5, Open Systems Interconnections; IEEE P1074.1, Software Lifecycle Processes working group; IEEE Mass Storage group; IEEE P1059, working group on the Standard of Software Verification and Validation; and IEEE 802.10, working group on Computer Communications, Security, and Privacy.

Laboratory staff are active on DOE committees and with the regional universities including participation in EXERSUG, ESNET steering and site coordinator's committees; chairing the ESNET network authentication task force and participating in the network authentication pilot program; co-chairing the Scientific Computing Information Exchange (SCIE) group of multiprogram laboratories; supporting the task group on DOE Standards, the DOE e:Mail task force, Distributed Computing Coordinating Committee for Energy Research, the task force for maximizing the effective use of Televideo Services and Assets, serving on the executive board and technical committee of NorthWestNet; and serving on the policy board and technical committee of the Concurrent SuperComputing Consortium.

Educational outreach includes the following: continued network design assistance to local school districts and Heritage College (a minority institution); providing ESNET access to local high schools in support of the National Educational Supercomputer program at NERSC; selected student Internet access under the Disabilities, Opportunities, Internetworking and Technology (DO-IT) program; and general access for teachers and students of the Kennewick, Washington, school district. These activities are supported by an excess personal computer installed outside the Laboratory firewall with dial access to local schools. The Laboratory continues, in cooperation with DOE-RL, to provide IBM computers, color monitors, and laser printers for a computer laboratory at Park Middle School in Kennewick under the Science Education Equipment Loan program.

Technology Transfer and Sharing Pacific Northwest National Laboratory's Chemical Management System (CMS), which maintains an inventory of all chemicals being held and their locations, was selected by DOE for transfer to Brookhaven National Laboratory under the Occupational Safety and Health Worker Protection Pilot Program and is also being transferred to Lawrence Berkeley Laboratory, the Idaho National Engineering Laboratory, and the National Renewable Energy Laboratory.

Software distributed to other sites included Software Scan license compliance program and database (DOEHQ-Human Resources); Popfon, an on-line, network based telephone directory system, to DOE-SAN and the Department of Defense; and MEPAS, a multimedia environmental pollutant assessment system was transferred to a small college for use in a degree program to train workers in waste site cleanup.

Software commercialized includes ReOPt, a remedial operations code which provides information on nearly 700 environmental contaminants and 88 remediation technologies; CINDY, an internal dosimetry code; TEMPEST, a thermal hydraulics code; and ChernoLit, a bibliographic database on the Chernobyl accident. 


\section{Major Goals and Near-Term Strategies}

\section{Infrastructure}

The Laboratory's network strategies are guided by the Information Technology vision established for the Integrated Computing Environment mentioned earlier. These include

- establishing and maintaining local area, Hanford Site, and Internet connectivity for all appropriate staff at all local and remote locations

- ensuring that the stability and reliability of the Integrated Computing Environment infrastructure is uncompromised; that network performance is acceptable

- continuously evaluating emerging technology and adding local area and Internet functionality that makes the infrastructure transparent, easy to use, adequately protected, and supports emerging applications (e.g., multimedia).

We neared a connectivity goal so only a modest number of new connections were added in FY 1995. However, an additional 200 to 400 connections will be added for the EMSL facility which will be completed in FY 1998.

The main focus in the next 2 years will be continuing to improve the reliability and performance of the network and continuing to expand its functionality. Following are planned activities: the oldest segments of the cable plant will be replaced, replacement of older network devices with systems that can be remotely monitored and controlled, completing the installation of Uninterruptible Power Supplies for primary routers and hubs, adding more personal computer and Macintosh servers to improve performance, complete the transition from OS/2 to NT Advanced Server, and add a redundant X.500 directory server. An additional FDDI ring, dedicated to application server file transfer, graphics, and replication services for distributed applications, will be added to improve performance.
Upgrades and/or new functionality being added includes Windows Internet Naming Services; move to Windows 4.0; implement pager access from the e:mail system; add redundant e:mail gateway to the Internet; enhance capability to publish to the Gopher server and WWW server without external intervention; upgrading PC/Macintosh/UNIX integrated file services, e:mail and calendar/ scheduler services, network management services, network measurement, and distributed password moderator.

A key strategy is to further downsize the business systems infrastructure migrating the few applications that remain on the IBM mainframe and replacing the Sequent IRM database system with dual Sparc servers. This project establishes the required infrastructure to support implementation of a data warehouse and migrate existing applications off the mainframe environment over the next 2 years.

Scientific and Engineering Computing Our strategies for scientific computing are aimed at meeting $\mathrm{DOE}$ and national needs with the most cost-effective technology consistent with the Laboratory's mission, program requirements, current scientific and engineering challenges, and the state of technology. Consequently, high-performance workstations, workstation clusters, and moderate to massively parallel computers with effective network access and bandwidth are key requirements for progress.

While the demand for high-performance computing at the Laboratory has more than quadrupled since FY 1988, workload distribution has shifted dramatically. Less demanding workloads have migrated to high-performance workstations and clusters while more demanding codes continue to be run at remote computer centers with highperformance resources (NERSC, Florida State, Cal-Tech, and LANL). At the same time, the most advanced codes are being redeveloped for massively parallel systems. We are aggressively pursuing further expansion in the demand for advanced parallel computing with EMSL taking a lead position in acquiring advanced computational resources. In FY 1994, the EMSL installed a KSR2 parallel architecture computer for software development as well as a prototype database/ archive computer. Implementation plans and specifications have been developed for a production database/ archive system and a massively parallel production system. These systems will be acquired in the first half of FY 1996.

The functionality of existing database systems continues to be expanded. TWINS has moved to production status and has been upgraded to add an issues management module. HEIS has been expanded, adding a Hanford Tank Characterization Database, increasing the range of analytical and physical samples stored and making them available either from HEIS or TWINS. The Laboratory is also developing the requirements definition for the successor system to HEIS which will further expand and integrate the wide variety of data, information, and systems that capture, store, manage, display, and report environmental information at the Hanford Site.

Near-term strategies in systems development will continue to stress the applications areas described earlier. Key strategies will be to continue refining, validating, and extending the wide range of scientific codes, models, and simulations which support DOE's mission requirements, the environmental cleanup activity at Hanford, Energy Research, and other DOE programs.

Business Information Systems Pacific Northwest National Laboratory's strategies for business information systems are focused in four key areas for FY 1995. These are

- develop and implement an integrated information management environment that empowers staff to conduct their work in a quality, timely, and cost-effective manner 
- develop and implement well-defined information management systems which meet the Laboratory's needs in conducting the activities of the business efficiently

- develop and implement the resources and expertise that will assist the Laboratory to become recognized for innovative, effective, and efficient use of information technology resources.

A key to establishing an integrated information management environment is the completion of the information architecture project initiated in FY 1994. The architecture will describe the Information Technology environment in terms of an applications, data, and technology architecture.

The primary business infrastructure activity is to replatform Pacific Northwest National Laboratory's business systems. Applications currently running on the Hanford mainframe and the Laboratory's midrange Sequent database are being rehosted to run on a Sun Sparc multiprocessor platform acquired in FY 1994. This is the next step in a continuous process of platform downsizing which began in the early 1980s. At the same time, a data warehouse, or repository, will be established on the Sun to store and maintain enterprise data. The repository will get its input from the distributed transaction processing databases located on distributed file servers. It will support long-term storage and access, replication services for the transaction processing environment, and contain the enterprise data dictionary and applications inventory. This will enhance centralized control mechanisms while supporting the distribution of operational applications, and establish the basis for moving to a near-real-time administrative applications environment.

Another key to the business infrastructure is full implementation of electronic routing and authorization developed in FY 1993-1994. In FY 1995, DOE approval to use electronic routing and authorization for electronic purchase requisitions and employee time reporting was requested. This was key to being able to significantly reduce manual handling of paper and streamline the approval process by conducting much of the business activity electronically.

The main applications commercially acquired and/or developed in FY 1995 included a capabilities database and employee skills coding system, electronic staff advances and reimbursement, Laboratory core competencies, purchasing/subcontracts replacement, proposal pricing, employee work location, safety health information system, and an electronic policies, requirements, and procedures system.

Collaboration and Outreach The level of collaboration with and outreach to external organizations increased in FY 1994 through increased participation in DOE, industrial, $\mathrm{K}-12$ educational institutions, and university consortia; interaction with developers of advanced computer architectures; the high-performance computational chemistry initiative; new CRADAs; and through workshops sponsored by the Laboratory involving leading scientists in DOE, industry, and the university communities.

We expect to continue to sustain collaborations already in place and to initiate new ones depending on developing new requirements and seizing opportunities that materialize. Increased opportunities are expected to occur in the $\mathrm{K}-12$ educational outreach program which remains a high priority activity and for Internet collaboratories.

Resources and Initiatives There are four issues that need to be addressed to improve/enhance the Information Technology environment at the Laboratory. These are 1) significant increases in the reporting required under the revised IRM reporting process defined in the UNICALL; 2) significant increases in Headquarters oversight and review activities; 3 ) the continuing low levels of General Purpose Equipment capital funds; and 4) the continuing restriction on leasing computing resources.
The reporting requirements defined by the Information Management Crosscut Guidance in the Unified Budget call (UNICALL) impose significant new requirements. The most significant issue with the UNICALL is that the information required is not available in either FWP or ADS documents. It would appear that if these are important reporting requirements, then the most effective way to satisfy them would be to change the reporting required in the FWP and ADS processes to capture this data once and at its source. This would fully satisfy the programmatic reporting requirements of the UNICALL without significant additional cost.

In FY 1994, the Laboratory was required to devote significant resources responding to an increased level of inspection and evaluation, Headquarters IRM review, and local DOE-initiated oversight activities. Responding to these reviews at a time when the Laboratory is attempting to reduce cost has increased the cost of Information Technology Management without commensurate return on the investment.

The other two issues, which have been reported previously, continue to be the lack of General Purpose Equipment capital funds and the restriction on leasing computing resources. Since 1980 , research volume for DOE programs has grown from $\$ 74$ million to over $\$ 320$ million while general purpose equipment capital funds allocated to the Laboratory have remained constant at $\$ 1$ million. While technology downsizing and the continued decline in the cost of automated data processing equipment has partly ameliorated the lack of capital growth, increased demands for the network infrastructure, high-performance workstations, and advanced technology computers have worsened the situation.

Current restrictions on leasing equipment are further exacerbating the lack of capital funds in the Laboratory making it all but impossible to acquire the range of workstations, midrange, and high-performance systems needed. 


\section{Major Items of Equipment \\ (Budget Authorization \$ in Millions)}

\begin{tabular}{lcccccccc} 
& \multicolumn{9}{c}{ Fiscal Year } \\
& $\mathbf{1 9 9 4}$ & $\mathbf{1 9 9 5}$ & $\mathbf{1 9 9 6}$ & $\mathbf{1 9 9 7}$ & $\mathbf{1 9 9 8}$ & $\mathbf{1 9 9 9}$ & $\mathbf{2 0 0 0}$ & $\mathbf{2 0 0 1}$ \\
\hline Construction Capital Equipment $^{(2)}$ & 1.98 & 0.34 & 8.60 & 8.10 & 0.0 & 0.0 & 0.0 & 0.0 \\
Programmatic Capital Equipment (ER) & 1.2 & 0.25 & 0.15 & 5.0 & 5.0 & 2.0 & 20.0 & 5.0 \\
Total Funding & $\mathbf{3 . 1 8}$ & $\mathbf{0 . 5 9}$ & $\mathbf{8 . 7 5}$ & $\mathbf{1 3 . 1}$ & $\mathbf{5 . 0}$ & $\mathbf{2 . 0}$ & $\mathbf{2 0 . 0}$ & $\mathbf{5 . 0}$
\end{tabular}

(a) Construction Project EM-91-100.

In an increasing number of cases, scientific research and administrative applications are being developed to fit on existing platforms rather than being developed on the basis of the actual requirements either demanded, or made possible, by improved technology.

DOE should seriously consider options to make the best technology more readily available to the Laboratories either by A) eliminating the distinction between capital and operating funds for computing equipment, B) significantly raising the threshold for capital equipment to $\$ 50,000$ or higher, $C$ ) providing effective administrative procedures for leasing advanced technology equipment with fewer restrictions, or D) increasing the allocation of programmatic and general purpose equipment capital funds. In the Laboratory's case, increasing the general purpose equipment allocation to $\$ 1.5$ million a year would provide needed network resources and expansion of midrange administrative systems reducing operating cost and improving the performance of the infrastructure.

\section{Major Items of Equipment}

Major Items of Equipment have been requested through the Information Resources Management Long Range Site Plan for the Hanford Site. Through FY 1997 the Laboratory's proposed major items of equipment will be funded as part of Construction Project D-384, Environmental Molecular Sciences Laboratory.
Based on the goal of fielding a fully functional, equipped, and staffed research facility in FY 1997, we acquired a moderately parallel high-performance computer system in FY 1994 as a computational chemistry platform used to develop parallel versions of production chemistry codes, new algorithm, and computational chemistry support software. A massively parallel production system will be acquired in FY 1996 to provide the high-performance production environment as the research program progresses to full-scale operation. A prototype database/archive system was acquired in FY 1994. The production database/archive system will be acquired in FY 1996 to support longand short-term storage, database, and archive functions for both the experimental and modeling/simulation components of the EMSL research programs. A fiber optic network will be acquired in FY 1996-1997 as part of the EMSL infrastructure.

Acquisitions for modest upgrades of the EMSL high-performance computers (i.e., the database and production machines) are scheduled for FY 1997 and FY 1998. A small "experimental" machine is proposed in FY 1999. Acquisition of a replacement for the original high-performance, massively parallel production computer about 5 years after the original acquisition will be required if the facility is to maintain its state-of-the-art capabilities. In this plan, the replacement is shown in FY 2000, with expansion in FY 2001.
Capital resource requirements for these acquisitions are shown in the table.

\section{Site and Facilities}

\section{Laboratory Description}

The Pacific Northwest National Laboratory serves 1 ) as one of five Department of Energy multiprogram energy research laboratories, and 2) as the research and development center for the Hanford Site. Because of this dual role, the Hanford Site Landlord Program, under the purview of the Office of Environmental Management, is responsible for general purpose facilities and equipment primarily supporting Hanford Site mission activities. The Multiprogram Energy Laboratories-Facilities Support (MEL-FS) program, under the purview of the Office of Energy Research, is responsible for multiprogram facilities and equipment.

\section{Pacific Northwest National} Laboratory consists of a collection of buildings measuring approximately 195,000 square meters (2.1 million square feet) with assigned responsibility as follows:

- DOE-owned facilities 91,000 square meters ( 0.975 million square feet)

- Battelle-owned facilities 46,000 square meters $(0.5$ million square feet)

- DOE-leased facilities 14,000 square meters ( 0.15 million square feet) 
- Battelle-leased facilities 44,000 square meters ( 0.475 million square feet).

Through formal arrangement with the DOE, these facilities compose the Consolidated Laboratory and are used by over 3,800 Battelle employees to perform work for the DOE, other federal agencies, and private industry.

The Laboratory space distribution table illustrates that the Laboratory's Richland DOE facilities are located primarily in two areas. Most DOEowned facilities are located in the Hanford Site's 300 Area. DOE-leased space is located in the city of Richland, Washington, adjacent to the 300 Area site and Battelle private facilities. The 300 Area occupies approximately 4 square kilometers (1.5 square miles or 960 acres) in the southeastern portion of the Hanford Site, along the west bank of the Columbia River. The other DOEowned facilities are located in different operating areas on the Hanford Site.

The remaining graphics and text focus on the 104 DOE-owned buildings. The figure showing the age of Laboratory buildings illustrates that 94 percent of the Laboratory's 79 active DOEowned facilities are over 20 years old, and 52 percentare over 30 years old. All of the Laboratory's strategically important laboratories, as well as the major office/support facility, are now over 20 years old. Only 872 square meters $(9,388$ square feet) are less than 10 years old. The average age of the 79 active DOE-owned buildings is 29 years.

The figure showing the condition of Laboratory space by functional unit illustrates in greater detail the condition and function of all of the Laboratory's facilities. By far, research and development space is the predominant type of space at the Laboratory. Included in this category are laboratories (both nuclear and non-nuclear) for chemistry, materials, environmental and biological research, computation, electrical/electronics, applied physics, and general purpose research. The remaining functional types of space include administrative, accelerator, service, storage, and other.

\section{FY 1995 Laboratory Space Distribution}

\begin{tabular}{lcc} 
Location & $\begin{array}{c}\text { square feet } \\
\text { (million) }\end{array}$ & $\begin{array}{c}\text { Area } \\
\text { square meters } \\
\text { (million) }\end{array}$ \\
\hline Main Site (300 Area) & 0.975 & 0.091 \\
Leased (Offsite) & 0.15 & 0.014 \\
Total & 1.125 & 0.105
\end{tabular}

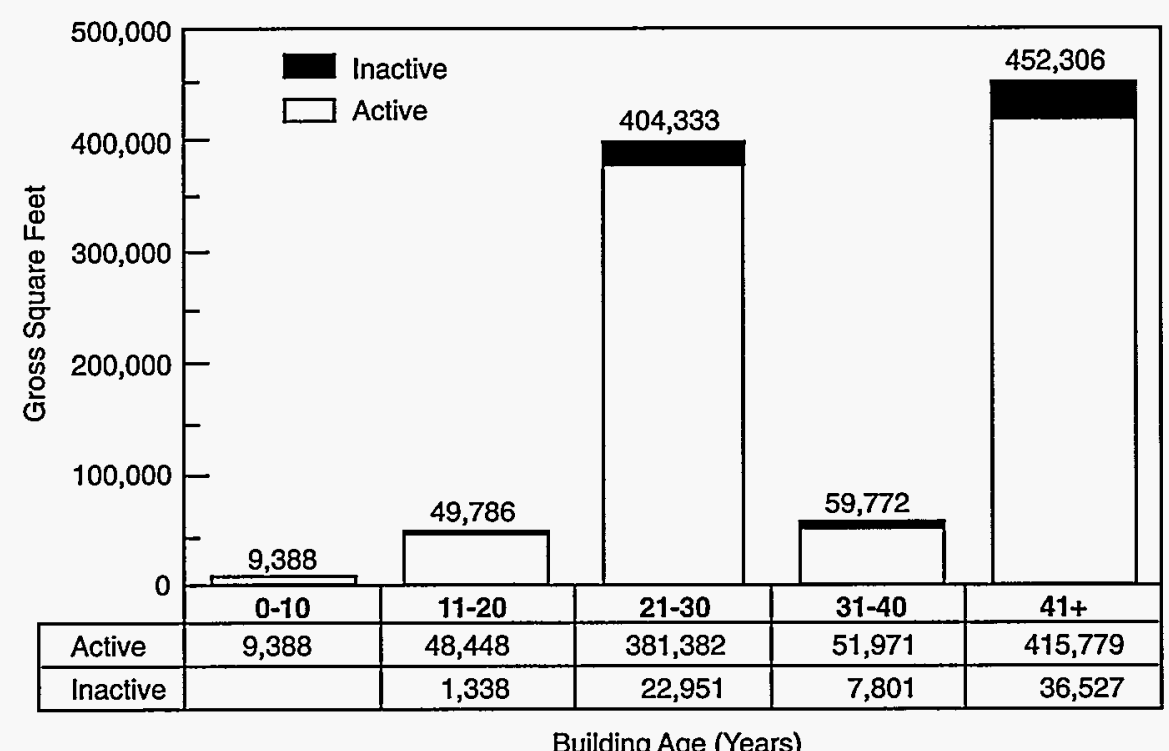

Laboratory area and age distribution (years).

As programs and research methodology change, the look, feel, and function of laboratory space must also evolve. In some instances, existing laboratory space is outdated or deteriorated, while in other cases it is not located near other related work or support space. The Laboratory's long-term facility strategy has been to consolidate in the south end of the 300 Area in renovated and replacement facilities. Funding is being sought through line item requests to the cognizant Secretarial Offices to accomplish these needed renovations to turn inadequate or under-used space into modern laboratories. Replacement laboratory space is being sought in instances where the current building's life cycle is at a practical end or the location of the facility is counter to long-term planning or impacted by other Hanford activities.
The Laboratory's DOE-owned office space is concentrated in one permanent facility and 21 trailers, as well as some office space within laboratory buildings. Opportunities to relocate staff out of some substandard trailer space will occur in FY 1996. The 337 Office Building is 22 years old and will require renovation in the near future.

The remaining space is a mixture of shops, storage buildings, and miscellaneous unique facilities (such as a technical library), service buildings, and environmental monitoring stations. The condition of the majority of the space is considered adequate. There are plans to replace several small, aged, and scattered storage buildings with a new facility after FY 2001. The Laboratory's facilities inventory also includes a collection of other structures such as 


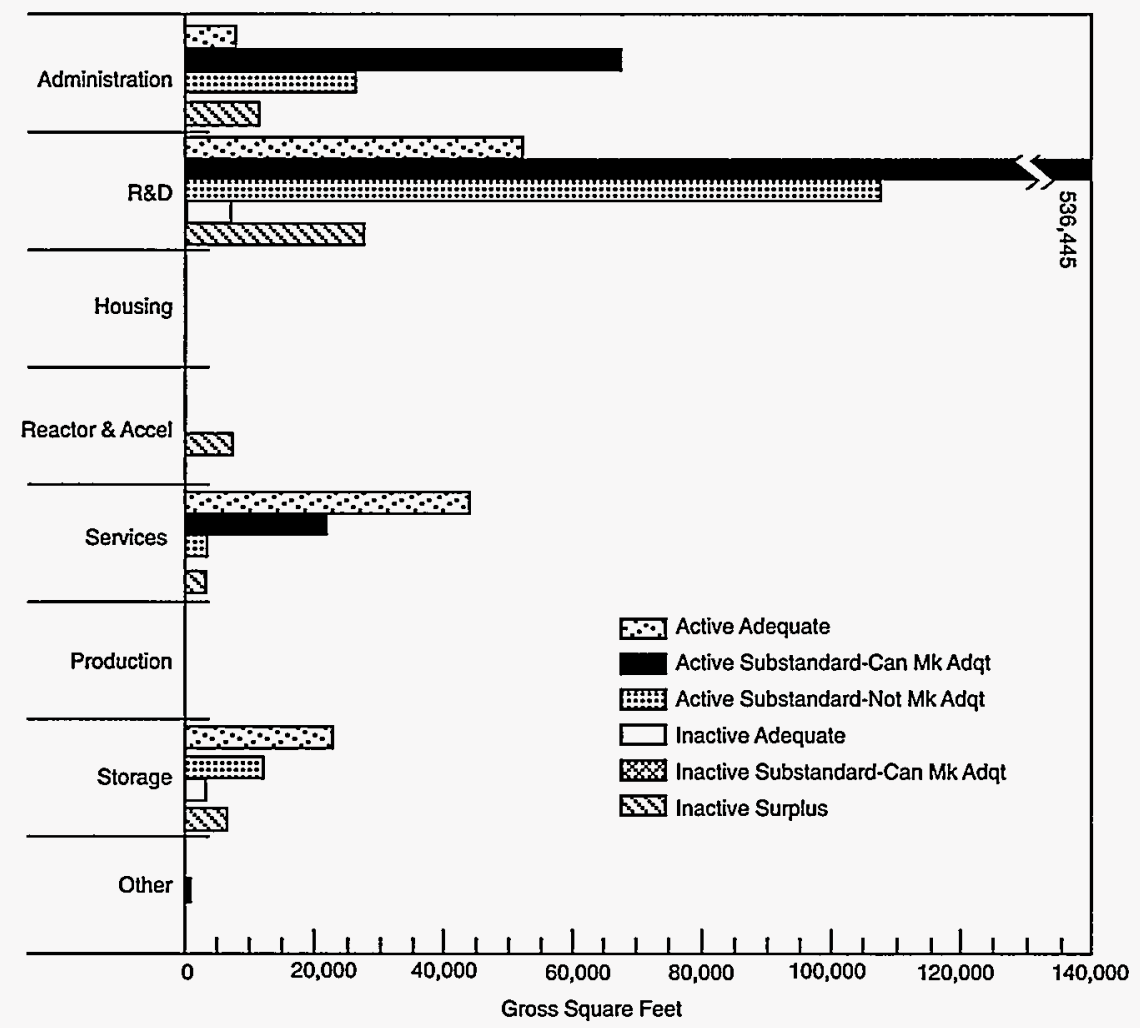

\begin{tabular}{|c|c|c|c|c|c|c|}
\hline & $\begin{array}{c}\text { Active } \\
\text { Adequate }\end{array}$ & $\begin{array}{c}\text { Active } \\
\text { Substandard } \\
\text { Can Mk Adqt }\end{array}$ & $\begin{array}{c}\text { Active } \\
\text { Substandard } \\
\text { Not Mk Adqt }\end{array}$ & $\begin{array}{l}\text { Inactive } \\
\text { Adequate }\end{array}$ & $\begin{array}{c}\text { Inactive } \\
\text { Substandard } \\
\text { Can Mk Adqt }\end{array}$ & $\begin{array}{l}\text { Inactive } \\
\text { Surplus }\end{array}$ \\
\hline $\begin{array}{l}\text { Administration } \\
\text { R\&D } \\
\text { Housing } \\
\text { Reactor \& Accel } \\
\text { Service } \\
\text { Production } \\
\text { Storage } \\
\text { Other }\end{array}$ & $\begin{array}{r}7,392 \\
52,845 \\
\\
44,307 \\
23,290 \\
352\end{array}$ & $\begin{array}{r}69,907 \\
536,445 \\
21,431\end{array}$ & $\begin{array}{r}26,156 \\
108,536 \\
3,862 \\
12,445\end{array}$ & $\begin{array}{l}7,159 \\
3,652\end{array}$ & & $\begin{array}{r}12,234 \\
27,635 \\
\\
8,021 \\
3,200 \\
6,716\end{array}$ \\
\hline Total & 128,186 & 627,783 & 150,999 & 10,811 & & 57,806 \\
\hline
\end{tabular}

Condition of Laboratory space by functional unit.

\begin{tabular}{|c|c|}
\hline \multicolumn{2}{|c|}{$\begin{array}{c}\text { Facilities Replacement } \\
\text { Value }\end{array}$} \\
\hline Facilities Type & $\begin{array}{l}\text { Replacement } \\
\text { in FY 1995 } \\
\$ \text { (millions) }\end{array}$ \\
\hline Buildings & 400 \\
\hline Utilities & 5 \\
\hline All Other & 6 \\
\hline Total & 411 \\
\hline
\end{tabular}

Laboratory. New replacement values have been computed based on industry standard indexes determined to be appropriate for facilities. Significant, additional replacement value is represented by the Hanford landlord utilities infrastructure inventory, but that responsibility is assigned to the Hanford management and operations contractor, not to the Laboratory.

\section{Facilities Plans and Options}

A national center for science and technology in the Pacific Northwest is being built around Pacific Northwest National Laboratory. This center, the Tri-Cities Science and Technology Park, integrates capabilities for basic science, applied research and development, and technology applications. The Park (see the figure) encompasses the DOE facilities in the southern portion of the 300 Area; the research and development laboratories owned by Battelle in the south, as well as Port of Benton and Washington State University Tri-Cities facilities; a total of 50 companies and organizations employing 8,000 people.

The interests of both government and industry are actively represented. Federally developed technologies are effectively transferred to the public and private sectors. The activities of the Park encourage the local economy to diversify, thereby reducing the historical dependence on DOE operations at Hanford. Regional business leaders, small local businesses, state officials, and federal agencies are working together to ensure that industry and government are aware of the unique technical, economic, managerial, and geographic assets that are available at Hanford. The focus remains on the diverse development of the 2,600 acresof land within the Park boundaries.

The 300 Area is within the northern boundary of the Park. This area is composed of DOE-owned facilities. The 300 Area Site Development Plan calls for locating new laboratory facilities in the southern portion of the area, demolishing the older facilities in the north and restoring their sites. 
The DOE budget reductions and the Laboratory's aggressive productivity actions have accelerated these plans. A dedicated Facility Transition Project Team has been established to expedite the specific facility consolidation and closure actions. Concurrently, the 300 Area utility infrastructure is being transitioned to the City of Richland and other private utility providers.

\section{General Purpose Facilities Plans}

All of the Laboratory's facilities are considered general purpose. This means the facilities and equipment are used by two or more DOE programs. The Laboratory's future development plans and options are inseparably linked with national DOE strategies, the Energy Policy Act of 1992, the Facilities Management 48 CFR Part 970, and guided by strategic initiatives being pursued at Hanford. By focusing on health and safety of the public and workers, and focusing on the other vital elements of these plans as well (energy, environment, education, economy, and national security), the Laboratory will achieve its vision of becoming a national center for science and technology. Our planning emphasis is to

- ensure that facilities are operated and maintained in full compliance with all applicable laws and regulations

- plan for and provide safe and operable facilities to accommodate and support ongoing and new research activities

- operate and maintain facilities economically to provide for the safety and health of plant personnel, protect the public and environs, protect property, and support research needs

- concentrate scarce financial resources on strategically important facilities that support overall Laboratory core competencies

- link, integrate, and unify the Laboratory's strategic and program plans with facility and site development plans and with specific design and construction projects

- improve the use of existing space and continue to thoroughly examine appropriate adaptations of existing space to new business areas

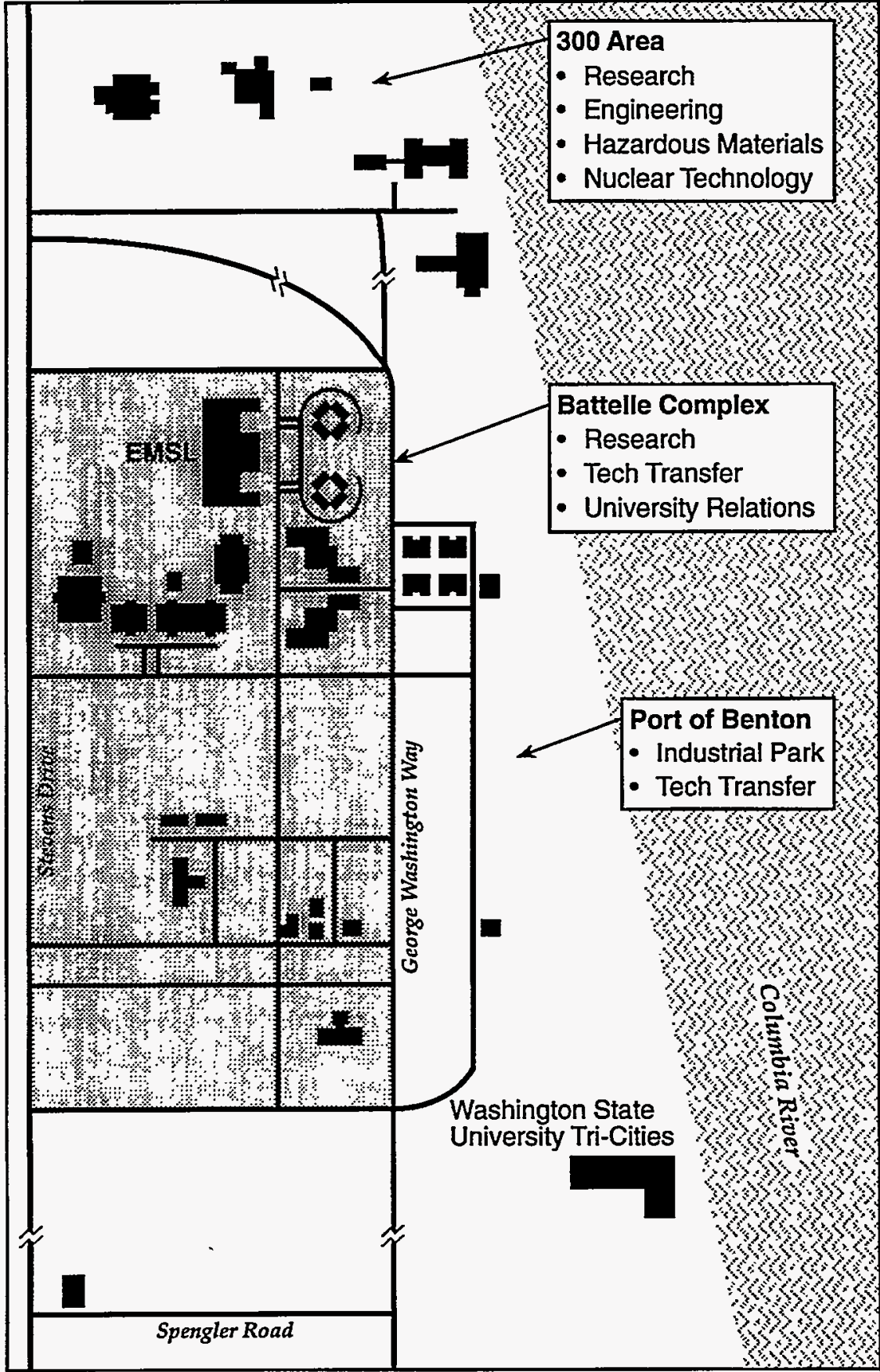

The Tri-Cities Science and Technology Park encompasses the 300 Area to the north and WSU-TC facilities to the south.

- consolidate similar technical functions wherever appropriate.

In recent years, facility upgrades have been focused on solving four general problem areas: 1) increased compliance with environmental, safety, and health regulations; 2) infrastrucure deterioration; 3) changing purpose of some facilities; and 4) shortage of adequate laboratory and office space. Progress to date in each of these areas follows.

Environment, Safety, and Health Regulatory Compliance The Laboratory has undergone numerous audits, reviews, inspections, and surveillances to assess the level of compliance with environment, safety, and health requirements. These include requirements 
derived from Washington State environmental laws, Environmental Protection Agency regulations, the $\mathrm{DOE}$, Occupational Safety and Health Administration standards, actions set forth in the Hanford Federal Facility Agreements (Tri-Party Agreement), and other environment, safety, and health assessments. The reviews focused on overall operations, documentation, agreements, planning, and the facility's performance.

The Environmental, Safety, and Health Management section in this plan identifies the results of these reviews. Facility-related deficiencies and proposed corrective actions have been included in several funding plans, and are described in this section under Facilities Resource Requirements.

Facilities Infrastructure Deterioration Modern facilities and equipment are essential to support a viable research program at the Laboratory. Consistent with our strategic objectives, a modernization plan is developing in conjunction with asset-based life cycle planning to anticipate facilities and infrastructure requirements for the next 20 years. Facilities infrastructure upgrades include exhaust system upgrades; heating, ventilating, and air conditioning control upgrades; fire protection compliance and fire alarm upgrades; as well as improvements to vacuum systems.

For other infrastructure components and facilities, the cost associated with major restoration approaches the cost of constructing a new replacement facility. The Laboratory has identified consolidation into existing facilities or replacement facilities as a more cost-effective approach. Consistent with the latest 300 Area Hanford Site Development Plan, these facilities are located in the southern-most portion of the 300 Area,adjacent to planned site infrastructure renewal. The connection to the City of Richland's sanitary sewer system is scheduled in FY 1996. The contract for 300 Area energy services is being competed in hopes of selecting an energy savings performance contractor early in FY 1996. The providers for electrical, water, fire protection, and other site services are also under review.

Mission Support To fulfill the Laboratory's mission, it is necessary to continue efforts to modernize facilities. Enhanced facilities are required to support our science, technology, and educational initiatives that will help $\mathrm{DOE}$ achieve its goals.

In keeping with the Laboratory's strategy to concentrate scarce financial resources on strategically important facilities that support overall Laboratory initiatives, facility improvements are focused on these major facilities. We have identified needed improvements to ensure compliance with environment, safety, and health requirements, and in providing adequate space for staff and operations.

Adequate Space The Laboratory has kept pace with its staff growth by using a lease-to-design-and-construct approach to provide four office facilities on Battelle-owned land. At the end of FY 1994, our shortage of adequate work stations was at 500 . Consolidation of staff into strategic facilities in response to FY 1995 downsizing resolved office space issues for the immediate future.

A key element in solving the problem of inadequate laboratory space is the renovation of aging labs and the replacement of labs that do not warrant the high cost of certain renovations. The pending consolidation to strategic facilities will also alleviate inadequate laboratory space issues.

\section{General Purpose Facilities}

Two programs provide major construction project line item support for the rehabilitation and replacement of the general purpose DOE-owned facilities assigned to the Laboratory. The programs are the Hanford Site Landlord Program under the purview of the Office of Environmental Management and the Multiprogram Energy
Laboratories-Facilities Support program under the purview of the Office of Energy Research. The construction projects include general use areas such as administrative and laboratory space. The Major Construction Projects table in a later portion of this section lists the General Purpose Facilities projects.

\section{General Plant Projects}

Discrete, ongoing corrective activities are required to bring facilities supporting the energy research and environmental management business areas into compliance with external air, surface water, and solid waste regulatory requirements, and internal DOE requirements. Representative projects include correcting Life Safety Code deficiencies, including upgrading stairwells, vertical openings, and improving exit corridors to current standards; air monitoring improvements; and cooling tower replacements, compressed air system replacement, HVAC controls replacement, and air conditioning upgrades. General plant project requirements are not duplicated in the construction line items. Pacific Northwest National Laboratory requests $\$ 3.5$ million per year from Energy Research for FY 1996-2001 multiproram general plant project requirements. From Environmental Management, the Laboratory requests $\$ 4.0$ million per year.

\section{General Purpose Equipment}

Pacific Northwest National Laboratory's multiprogram general purpose equipment requirements fall into two broad capital support categories: general purpose equipment for support services and nonprogrammatic equipment for functions such as safety, information systems networks and communications, security, and crafts. General purpose equipment is required for a basic, multipurpose laboratory capability to support many research activities.

The multiprogram general purpose equipment funding, provided at a fairly constant level in recent history, is 
being applied to meet priority requirements. Because funding was limited to $\$ 0.5$ million in FY 1995 , not enough is available for state-ofthe-art technological improvements beyond that needed to support priority items.

The Laboratory's Hanford Support general purpose equipment requirements are included in the Environmental Restoration and Waste Management FiveYear Plan. In general, environmental restoration and waste management general purpose equipment funding requirements are over $\$ 2.5$ million yearly.

\section{Facilities Resource Requirements}

The Laboratory's capital asset management process systematically identifies facility requirements, develops life cycle management plans for individual facilities, evaluates priorities of facility requirements, and initiates development of capital projects. The Multiprogram Energy Laboratories-Facilities Support program supports facility and site infrastructure planning activities. The Laboratory's capital facility requirements, as currently authorized and projected, are summarized in the Major Construction Projects table. The overall multiyear program plan

- is consistent with the DOE constrained budgetary guidance

- assumes a long-term effort will be necessary to fund and implement full requirements of the Laboratory

- emphasizes a commitment to compliance with operational, environmental, safety, health, and security standards, and a significant role for the Laboratory research and development efforts for the DOE and the nation.

The following program and funding profiles were developed to establish a balance between high priority work and the realities of the current federal budget climate. The Laboratory's project priority setting process uses a DOE-developed prioritization methodology, Capital Asset Management Process (CAMP). The deficiencies, purpose, and needs of each project are evaluated using guidance criteria given in one or more of four basic categories: Health and Safety, Environment/Waste Management, Safeguards and Security, and Programmatic. A numerical rating is assigned to each activity. This rating allows a relative ranking to be established between the various competing activities. This prioritization methodology is the basic tool used to objectively aid the decision-making processes in weighing the relative importance of our facility improvement projects.

Developing and implementing the Laboratory's coordinated multiyear integrated facilities plan is significant because the funding of DOE programs at the Laboratory is relatively diverse and the program relationships at the Hanford Site are complex. The Laboratory needs the institutional support of both Energy Research and Environmental Management to provide an adequate basis for the Laboratory to best serve DOE's needs. The following discussion summarizes our specific program plans and requirements. NEPA documentation has been approved unless otherwise noted.

Programmatic Line Item Construction Funding Pacific Northwest National Laboratory has one programmatic line item construction project, the Environmental Molecular Sciences Laboratory.

\section{Environmental Molecular Sciences} Laboratory (91-EM-100) The Environmental Molecular Sciences Laboratory will be a 19,000 -square-meter (200,000 square foot), state-of-theart research facility. It will support basic and applied research in the environmental molecular sciences. A wide range of research will be performed in the EMSL to develop new knowledge and technologies that, when implemented, will reduce the cost and increase the effectiveness of environmental restoration and waste management efforts undertaken at Hanford and other DOE sites. The facility will house approximately 200 permanent building occupants and 60 visiting scientists and includes a seminar area that will accommodate 100 people.
Multiprogram Energy LaboratoriesFacilities Support Program The Multiprogram Energy LaboratoriesFacilities Support program is designed to maintain infrastructure integrity at the DOE's multiprogram energy laboratory facilities. The strategy of the MEL-FS program is to maintain operations in a safe, cost-effective, and productive manner; reduce the backlog of facilities deficiencies; address environment, safety, and health remediation needs; and provide resources to manage surplus facilities related to Energy Research activities. The MEL-FS program is composed of three subprograms from which the Laboratory requests funding support

- General Purpose Facilities (GPF)

- Environment, Safety, and Health Support

- Inactive and Surplus Facilities.

General Purpose Facilities Subprogram The General Purpose Facilities subprogram provides construction support for the rehabilitation and replacement of general purpose facilities. These construction projects are directed at general purpose facilities which include general-use service and support facilities such as administrative space, general office/laboratory space, and utility systems. The General Purpose Facilities subprogram also provided operating funds to support Laboratory general purpose facilities planning and management activities. These activities included implementing the condition assessment surveys, documenting asset life cycle plans, and preparation of integrated facilities plans. The table identifies the Laboratory's most acute general purpose facilities requirements. Direct DOE budget reductions have caused us to reconsider proposed outyear projects. Efforts are under way to identify FY 1998 projects. A brief decription of the two major ongoing construction projects are listed below.

Safety Compliance Modifications, 326 Building (92-E-601) This ES\&Hdriven FY 1992 line item has a total estimated construction cost of $\$ 8.5$ million. 


\section{Major Construction Projects - FY 1996-2001 Planning Period \\ (Budget Authorization \$ in Millions)}

\begin{tabular}{cccccccccc} 
& \multicolumn{1}{c}{ Fiscal Year } \\
$\begin{array}{c}\text { Total Est. } \\
\text { Const. Costs }\end{array}$ & 1993 & 1994 & 1995 & 1996 & 1997 & 1998 & 1999 & 2000 & 2001 \\
\hline
\end{tabular}

\section{FUNDED CONSTRUCTION}

\section{General Plant Projects}

General Plant Projects (KP) ${ }^{(a)}$

General Plant Projects EM 60 (EW) ${ }^{(2)}$

Environmental Management Projects (EW)

329 Building Compliance

325 Facility Compliance/Renovation

Multiprogram Energy Laboratories-

Facilities Support Program Projects (KG)

Safety Compliance Modifications,

326 Building

Life Safety Code Compliance

Program Related Projects

Environmental Molecular Sciences

Laboratory ER (KP)

Total Funded Construction

BUDGETED CONSTRUCTION

General Plant Projects

General Plant Projects (KP)

General Plant Projects EM 60 (EW)

Multiprogram Energy Laboratories-

Facilities Support Program Project (KG)

Multiprogram Laboratory Rehabilitation

Electrical Safety Rehabilitation

324 Facility Compliance/Renovation

Total Budgeted Construction

PROPOSED CONSTRUCTION

General Plant Projects

General Plant Projects (KP)

General Plant Projects EM 60 (EW)

Environmental Management Projects (EW)

RLWS Replacement \& Laboratory Support

Nuclear Facilities RLWS Upgrade

10.0

1.0

Total Proposed Construction

Total Construction

(a) Budget codes are identified in parentheses.

207.9

$2.0 \quad 2.8$

$3.5 \quad 3.5$

$\begin{array}{lll}9.9 & 2.5 \quad 2.6\end{array}$

$\begin{array}{llll}6.0 & 1.5 & 3.5 & 1.0\end{array}$

$\begin{array}{llll}8.5 & 3.0 & 1.9 & 1.9\end{array}$

$\begin{array}{llll}2.0 & 0.5 & 1.0 & 0.5\end{array}$

$\begin{array}{lllll}28.5 & 32.0 & 40.0 & 50.0 & 35.1 \\ \mathbf{4 1 . 5} & \mathbf{4 7 . 3} & \mathbf{4 3 . 4} & \mathbf{5 0 . 0} & \mathbf{3 5 . 1}\end{array}$

1.3

2.0

$\begin{array}{lll}0.4 & 2.7 & 3.0\end{array}$

$\begin{array}{lllll}0.2 & 1.5 & 1.5 & 1.5 & 2.1\end{array}$

$\begin{array}{lll}1.5 & 3.5 & 0.8\end{array}$

$\begin{array}{lllll}5.4 & 7.7 & 5.3 & 1.5 & 2.1\end{array}$

$\begin{array}{llllll}2.1 & 3.5 & 3.5 & 3.5 & 3.5 & 3.5 \\ 1.6 & 4.0 & 4.0 & 4.0 & 4.0 & 4.0\end{array}$

\begin{tabular}{|c|c|c|c|c|c|c|c|c|}
\hline & & & & & & 1.9 & 6.0 & 2.1 \\
\hline & & & & & & 4.0 & 6.0 & \\
\hline & & & 3.7 & 7.5 & 7.5 & 13.4 & 19.5 & \\
\hline 41.5 & 47.3 & 48.8 & 61.4 & 47.9 & 9.0 & 15.5 & 19.5 & \\
\hline
\end{tabular}

$47.3 \quad 48.8$

$61.4 \quad 47.9$

$\begin{array}{lll}.0 & 15.5 & 19.5\end{array}$

9.6 
The purpose of this project is to ensure continuity of operations in a vital laboratory facility supporting Energy Research programs. The 326 Building figures prominently in research in structural materials, microstructural analyses, chemical methods and separations, component analysis, supercritical fluids, superconducting materials, and in various other basic research programs. This project will bring the 326 Building, which is an aged but strategically important laboratory, into compliance with DOE Order 6430.1A, General Design Criteria, NFPA requirements, National Electric Code requirements, and Washington State requirements.

\section{Multiprogram Laboratory Rehabilitation} (95-E-310) This Building Rehabilitation and Upgrade FY 1995 line item has a total estimated construction cost of $\$ 6.1$ million. The 331 Building, Life Sciences Laboratory, was designed to accommodate the care and use of large and small animals. Originally, the facility was dedicated to life-span animal research. Experiments included exposure to chemical carcinogens and radiation. Current and planned laboratory needs call for cell biology, tissue, and general chemistry type laboratories to support new programs related to areas of molecular biology. This type of research is already being performed on the third floor of the 331 Building in existing laboratory space that has been remodeled. It is advantageous to provide new laboratories adjacent or close to these. This project will replace the third floor small animal area with cell biology, tissue, and general chemistry type laboratories and consolidate animal care facilities on the first floor of the 331 Building. The 331A Building, adjacent to the main 331 Building, is currently vacant and in relatively poor condition. The building cannot be effectively remodeled to suit any current or future research need and therefore will be demolished.

\section{Environment, Safety, and Health} Support Subprogram The Environment, Safety, and Health Support subprogram provides support necessary to correct deficiencies identified in internal reviews and inspections that are related to DOE Energy Research facilities responsibilities. Comprehensive assessments initiated this subprogram, which examined environmental, safety, and health performance of DOE facilities. The assessments were conducted by a team of specialists from various $D O E$ offices, contractors, and consultants. The subprogram was designed to alleviate increases in laboratory overhead rates and General Plant Projects, General Purpose Equipment, and General Purpose Facilities funding levels associated with correcting selected deficiencies that were identified. The Laboratory's initial remediation action plan was successfully completed. A few capital construction projects continue in progress (see the following examples).

\section{Life Safety Code Compliance (93-E-317)} This ES\&H-driven FY 1993 line item has a total estimated construction cost of $\$ 2$ million and corrects immediate Life Safety Code compliance problems in several aged multiprogram laboratories. Affected buildings requiring near-term safety improvements include $306 \mathrm{~W}, 331,337$, and 3720 . Actions include protecting vertical openings, stairwell enclosures, and exit corridors to address OSHA issues.

The Life Safety Code specifies how buildings must be arranged and constructed to protect occupants in the event of an emergency evacuation. The code is mandated by DOE Order 5480.4 (Environmental Protection, Safety and Health Protection Standards) and DOE Order 6430.1A (General Design Criteria). Changes in the construction of Laboratory facilities are needed to comply with the requirements of the Life Safety Code. Failure to comply with the Life Safety Code jeopardizes the safety of staff members and visitors if emergency evacuation of a facility is needed. This project corrects OSHA deficiencies.

Electrical Safety Rehabilitation (95-E-303) This ES\&H-driven FY 1995 line item has a total estimated construction cost of $\$ 6.8$ million for replacing deteriorated wiring and outmoded equipment and eliminating unsafe operating conditions in several key multiprogram laboratories. Actions include rehabilitation of emergency power building distribution systems, motor control centers, and emergency lighting.

Affected buildings range from 20 to 40 years old and have electrical systems that are worn out and present a safety hazard. These improvements are required to provide building electrical systems that are safe, efficient, reliable, and maintainable as required by the DOE Order 5480.4 (Environmental Protection, Safety and Health Protection Standards) and DOE Order 6430.1A (General Design Criteria). The existing electrical systems do not meet the National Electric Code for safe working clearances, separation of cables, and shock prevention.

\section{Environment, Safety, and Health} Support Subprogram Capital Equipment Requirements Capital equipment requirements are driven by regulatory compliance or provide for a significant cost savings in operations. FY 1995 funding provided for equipment to reclaim and recycle chlorofluorocarbon refrigerants.

Environment, Safety, and Health Support Subprogram Operating Requirements At the request of the DOE Office of Energy Research, the Laboratory submitted its environment, safety, and health support operating requirements for MEL-FS facilities by proposal for funding in FY 1996 and FY 1997. It iscoordinated with requirements expressed in the Environment, Safety, and Health Management 5-Year Plan.

\section{Inactive and Surplus Facilities}

Subprogram Pacific Northwest National Laboratory's Inactive and Surplus Facilities 5-Year Plan provides for the identification, management, surveillance and maintenance, monitoring, characterization, cleanup, removal, and reutilization or transfer of all surplus general purpose facilities related to DOE Energy Research program activities. Some facilities will be cleaned up and reused, others will be removed to make way for new construction, while others will be transferred to the DOE Environmental Restoration's Decontamination and 
Decommission program. Environmental Assessments will be done on a facility-by-facility basis. The FY 1996 and FY 1997 projectscan be accomplished immediately upon funding since they are not dependent on other projects.

Specific facilities have been named, categorized, and prioritized through efforts including the DOE secretarial initiative Surplus Facility Inventory and Assessment Project; and initiation of the asset condition assessment surveys. This was accomplished consistent with the planning processes of the FY 1994 Laboratory Integrated Facilities Plan, the current Hanford Mission Plan, and the Hanford Site Development Plan update. To ensure specific surplus facilities are vacated, a rapid action team has been established at the Laboratory to manage the reconfiguration of space, relocation of staff and equipment, and disposition of vacant facilities.

The Laboratory is requesting operating funds for these activities via a field work proposal submitted to the MEL-FS Inactive and Surplus Facilities subprogram (KG-03). In FY 1995, MEL-FS KG-03 funding was used for the removal of 331F, Animal Care Storage Facility, and cleanout of the 331 Inhalation Suite for reuse. The Laboratory worked with the Hanford Site $M \& O$ contractor to excess the 331F Building in place. The building was auctioned with proceeds going to DOE. This process also enabled Laboratory to remove the 331J Building and Incinerator.

In FY 1996, a small amount of MEL-FS KG-03 funding will be carried over from the savings incurred from the removal of $331 \mathrm{~F}$ facility. This carryover will fund the removal of a glove box and DRI Train located in the 325 Building, room 52 , which may potentially be contaminated by sodium.

In FY 1997, proposed activities include the following:

- the removal of $6652 \mathrm{C}$ shed

- the removal and disposition of lead from 320 Building Laboratory 1
- disconnect and removal of fume hood from 331 Building, room 304

- the removal of White Bluffs Facility 1 and 2 storage buildings.

Environmental Management Funding The facilities that are not assigned as multiprogram facilities to Energy Research are primarily supporting the Hanford mission. The DOE Environmental Management Office is also the Hanford Site Landlord, and as such, is assigned funding responsibility for Laboratory's general purpose Hanford Support facilities $(318,324,325,327$, $3730,3760,622 \mathrm{R}$, and 747A). An assortment of trailers, small storage buildings, meteorological facilities, and some surplus facilities round out the Environmental Management Landlord inventory of 27 facilities.

These facilities support the Hanford Site environmental restoration and waste management mission, including meeting Tri-Party Agreement milestones, the Hanford Waste Vitrification program, the Tank Waste Remediation Systems, and Spent Fuel and Disposal Systems. Also, the Laboratory's site service responsibilities include environmental surveillance, monitoring, and oversight programs; preparation of major environmental documents; occupational dosimetry; radiological calibrations; operation of the whole-body counter; emergency preparedness; occupational radiation exposure records; and operation of the Hanford Technical Library.

DOE Environmental Management line item construction candidates are briefly described below. The construction projects are proposed in the Laboratory's 5-year (FY 1996-2001) DOE Environmental Management Program Plan.

\section{Building Compliance (91-E-322)}

The total estimated construction cost for this FY 1991 project is $\$ 9.9$ million The project renovates major building systems in an aged, but strategically important laboratory. Improvements include better ventilation, air filtration, and waste water piping to control radionuclides and ensure safe liquid effluent. These renovations are necessary to comply with the Clean Air Act, Clean Water Act, Resource Conservation and Recovery Act, and other requirements.

The project includes the following modifications to the 329 Building:

- upgrade the HVAC system to meet the requirements of 40 CFR 61 (National Emission Standard for Hazardous Air Pollutants); and to comply with the Best Available Radionuclide Control Technology requirement, and the State of Washington Administrative Code Chapter 402-80 (Monitoring and Enforcement of Air Quality and Emission Standards for Radionuclides)

- replace corroded service piping

- replace deteriorated waste piping systems to comply with DOE Order 5400.1 (General Environmental Protection Program) and with 40 CFR 116 (Federal Water Pollution Control Act), which require that hazardous materials cannot be released in a liquid effluent (extensive corroded piping holds the potential for releasing radioactive constituents)

- upgrade the fire protection system to meet NFPA 13

- replace a portion of the building electrical system to meet NFPA 70 (the National Electric Code)

- establish laboratory and corridor fire separation to comply with NFPA 101 , which is a mandatory requirement of DOE Order 6430.1

- provide access for the physically disabled to comply with 41 CFR 101.19.6 (Uniform Federal Accessibility)

- modify laboratories to relieve crowding and reduce safety risks.

The purpose of the project is to ensure continuity of operations in a vital laboratory supporting DOE business areas in environmental restoration. Significant analytical chemistry associated with Tri-Party Agreement Milestone 
M-10, and RCRA and CERCLA is performed in the facility. The laboratory houses part of the Chemical Measurement Section. This section includes the only Hanford Site laboratory staff qualified to meet EPA Contract Laboratory Program environmental measurements, an important part of the Tri-Party Agreement. The 329 Building also houses critical chemical laboratories and staff associated with method development research for DOE's waste management effort.

\section{Facility Compliance/Renovation} (93-D-184) This FY 1993 line item has a total estimated construction cost of $\$ 6$ million. The 325 facility is a vital laboratory. This project is necessary to support the continuance of radiochemical research and development, and the consolidation of 300 Area radiological work and closure of inadequate facilities. The project consists of the following actions: 1) provide fire separations for electrical switchgear; 2) replace electrical panels, powerconditioners, and switchgear circuit trips; 3) replace 17 Plexiglas glove boxes; 4) remove the B-Unit stack sampler; 5) upgrade the fire sprinklersystem; and 6) install a P-10 gas system.

\section{Facility Compliance/Renovation}

(95-D-454) This FY 1995 line item has a total estimated construction cost of $\$ 5.8$ million. This activity focuses on renovation of major building systems in an aged, but strategically important, Hanford Support laboratory. Much of the work in the building is associated with the DOE-EM business area including development and testing of new engineered systems and equipment for DOE's environmental and restoration programs and cleanup efforts at multiple DOE, DOD, and industrial solvent, heavy metal, and mixed waste sites. Facility capabilities include hot cells, nonradioactive laboratories, and high-bay space for bench- to full-scale prototype work. The project includes the following actions: 1) upgrade/ replace HVAC systems; 2) replace contaminated piping systems;

3) upgrade the sprinkler system;

4) removeand replace large areas of asbestos; and 5) replace deteriorated interior building facilities such as electrical panels, electrical equipment, and other building equipment.

Radioactive Liquid Waste System Replacement and Laboratory Support Facility (99-D-XXX) EM-60 This FY 1999 line item has a total estimated construction cost of $\$ 10$ million and will provide upgrades to the radioactive liquid waste system (RLWS) that serves the 325 " $\mathrm{B}$ " hot-cell complex, which is critical to functionality of this strategic facility.

\section{Nuclear Facilities RLWS System Upgrades (99-D-XXX) EM-60 This} FY 1999 line item totals $\$ 6.5$ million and will provide an upgrade to the RLWS capability in the Laboratory's nuclear facilities.

Maintenance Funding The Laboratory recognized the $\mathrm{DOE}$-wide effort to provide maintenance funds on a life cycle basis in response to asset-based assessment and planning efforts. The program included establishing standards, methods, procedures, data collection systems, database architecture, and training, along with life cycle surveys of facilities to establish maintenance requirements. The Laboratory will continue to be faced with tightening budgets that require better prioritization, cost control, and management of maintenance. In response, the Laboratory will rely on a graded approach to assist the decision-making process in managing limited maintenance resources.

\section{Consolidated Information Center}

The Hanford Technical Library, managed by Pacific Northwest National Laboratory as a Hanford Site service for the DOE, represents a unique information resource. Its staff provide an integrated scientific and technical information resource and services. They will continue to be major contributors to effective information management. The main library is located within the 300 Area on the Hanford Site.
More accessible, but less comprehensive, is the library at the Washington State University Tri-Cities campus, adjacent to the Laboratory. Pacific Northwest National Laboratory has proposed consolidating the two libraries, thereby minimizing duplication and overlap through the use of shared staff and collections. This will benefit Hanford staff, visiting scientists, WSU faculty and students, and the general public. The consolidation will also allow the joint library to provide evening and weekend services.

The consolidated library would be located in leased space in a new building on the WSU-TC campus called the Consolidated Information Center. The new building would also include WSU's Conferences and Institutes and an Environmental and Technology Resource Center managed by WSU. The library facility would be operated by the Laboratory, in conjunction with WSU-TC. The DOE Public Reading Room, which is currently managed by the Laboratory and located on the WSU-TC campus, would also be located in the new library.

The Washington State Legislature has appropriated $\$ 1.4$ million for the architectural design of the facility. The DOE and General Services Administration have agreed in principal on a 20-year lease for part of the facility. Funding for the Center has been approved in the 1995-97 Washington State Legislative budget. The construction budget provides $\$ 9.7$ million for the library structure with the rest of the funding coming through the DOE lease agreement. The project is expected to be completed in spring 1997.

The Consolidated Information Center would be tied into the Hanford Local Area Network so staff and users would have full access to the electronic library services that are currently provided, as well as to additional services that may be added. 


\section{Consolidated Information Center}

\section{(Budget Authorization \$ in Millions)}

\begin{tabular}{lcccccc} 
& \multicolumn{7}{c}{ Fiscal Year } \\
Current Programs & $\mathbf{1 9 9 5}$ & $\mathbf{1 9 9 6}$ & $\mathbf{1 9 9 7}$ & $\mathbf{1 9 9 8}$ & $\mathbf{1 9 9 9}$ & $\mathbf{2 0 0 0}$ \\
\hline Operating & 2.8 & 2.4 & 2.5 & 2.5 & 2.6 & 2.6 \\
Move and Relocation & 0.0 & 0.0 & 0.4 & 0.0 & 0.0 & 0.0 \\
Capital Equipment & 0.0 & 0.1 & 0.1 & 0.1 & 0.1 & 0.1 \\
Total & 2.8 & $\mathbf{2 . 5}$ & $\mathbf{3 . 0}$ & $\mathbf{2 . 6}$ & $\mathbf{2 . 7}$ & $\mathbf{2 . 7}$
\end{tabular}

(a) Construction Project EM-91-100.

The consolidation of the holdings of the Hanford Technical Library and the WSU-TC library, and the increased accessibility of electronic information would create a unique and very valuable set of resources. This consolidation would also make the resources available to a much larger community and would make the Laboratory's research results more available to WSU students and faculty and to the public. The consolidation is an important local ingredient for building and supporting an infrastructure that contributes to and is part of the DOE's science education and technical information foundation.

The primary incremental budgetary impacts would be the building lease cost for the library space (similar to current costs) and the one-time costs associated with the move to the new facility.

\section{Summary}

Pacific Northwest National Laboratory has made significant contributions to the Hanford long-term planning efforts as well as optimizing tactical actions taken to respond to DOE budget reductions, downsizing, and changing programmatic requirements. The Laboratory has partnered with other Hanford Site contractors, DOE, and local and state government to plan for the Hanford of tomorrow, and is contributing to the 300 Area Energy Savings Performance Contract and the transition of 300 Area utility infrastructure to the City of Richland. Within the Laboratory, significant headway has been accomplished in maximizing facility use and reducing costs. In particular, a 300 Area Consolidation Strategy has been developed and implemented, actions are under way to move 325 Building radiological operations from a Category $I I$ to Category III level, a space charge-back system has been implemented for FY 1996, and facility specific use agreements are being implemented.

These actions are directly linked to accomplishing the objectives set forth in Pacific Northwest National Laboratory's six critical outcome areas, which form the basis for the Laboratory's FY 1996 performance appraisal contract with DOE.

\section{Communication and Trust}

Pacific Northwest National Laboratory is supporting DOE in its continuing transformation to a new culture by emphasizing communication and trust as an underlying value at the Laboratory. For the Laboratory to maintain credibility as a DOE national multiprogram laboratory, we must be viewed internally and externally as an organization where trust and respect are pervasive and good communication is second nature.

Effectively communicating information and building trust with our staff, our customers, and our stakeholders is essential in the Laboratory's pursuit to become a world-class laboratory.

Successful managers at the Laboratory listen to customers, staff, and stakeholders, and work with them in teams characterized by openness and trust, risk-taking, and readiness to explore new ideas.

Our commitment to the effective management of scientific and technical information is a critical component of every research project and program, both as input and as output, and we intend to continue managing the use of such information efficiently. We emphasize the rapid transfer of knowledge to the scientific and public communities, using a variety of formats and channels, in support of DOE's mission. Planning for information management activities will continue to be integrated into our planning and budget processes by our program and project managers in partnership with DOE programmatic staff. We will continue to emphasize effective delivery of scientific and technical information as an important outcome of our research.

The Laboratory's efforts to improve communications and build trust include

- timely and accurate communication of information to help staff make informed decisions

- active participation across the Laboratory in planning and implementing activities to achieve quality and improve productivity

- communication of the Laboratory's accomplishments internally, locally, regionally, and throughout the United States

- communication of the benefits of science to the public to help them appreciate the value of the science and technology investment to meeting national needs

- commitment to the effective life cycle management of scientific and technical information and its key role in promoting the DOE's missions and in creating an informed public. 
The resource requirements of research and development for Hanford Site support are included in the resource projections of the various funding programs. Research and development for other DOE sites at other DOE facili- ties; however, is shown as a separate program. The resource projections for FY 1995 are actual values.

\begin{tabular}{|c|c|c|c|c|c|c|c|c|}
\hline \multicolumn{9}{|c|}{$\begin{array}{l}\text { Laboratory Funding Summary } \\
\text { (Budget Authorization \$ in Millions) }^{(a)}\end{array}$} \\
\hline & 1994 & 1995 & 1996 & $\begin{array}{l}\text { Fisca } \\
1997\end{array}$ & $\begin{array}{c}\text { Year } \\
1998\end{array}$ & 1999 & 2000 & 2001 \\
\hline DOE Effort & 404.4 & 446.4 & 344.3 & 383.8 & 413.5 & 433.8 & 479.5 & 471.3 \\
\hline Work for Others & 75.3 & 59.4 & 75.0 & 79.0 & 82.0 & 84.0 & 86.0 & 88.0 \\
\hline Total Operating & 479.7 & 505.8 & 419.3 & 462.8 & 495.5 & $\mathbf{5 1 7 . 8}$ & 565.5 & 559.3 \\
\hline Capital Equipment & 23.6 & 20.1 & 18.6 & 23.8 & 24.5 & 18.8 & 38.9 & 19.0 \\
\hline General Purpose Equipment-GPE & 1.5 & 1.2 & 0.9 & 0.9 & 0.9 & 0.9 & 0.9 & 0.9 \\
\hline General Plant Projects-GPP & 6.3 & 1.1 & 6.0 & 7.5 & 7.5 & 7.5 & 7.5 & 7.5 \\
\hline Landlord Line Items & 6.1 & 2.5 & 3.5 & 0.8 & 0.0 & 5.9 & 12.0 & 2.1 \\
\hline Program Line Items & 32.0 & 40.9 & 50.0 & 35.1 & 0.0 & 0.0 & 0.0 & 0.0 \\
\hline MEL-FS'b) Construction & 2.9 & 3.0 & 4.2 & 4.4 & 2.6 & 6.9 & 3.8 & 0.0 \\
\hline Total Laboratory Funding & 552.1 & 574.6 & 502.5 & 535.3 & 531.0 & 557.8 & 628.6 & $\mathbf{5 8 8 . 8}$ \\
\hline
\end{tabular}

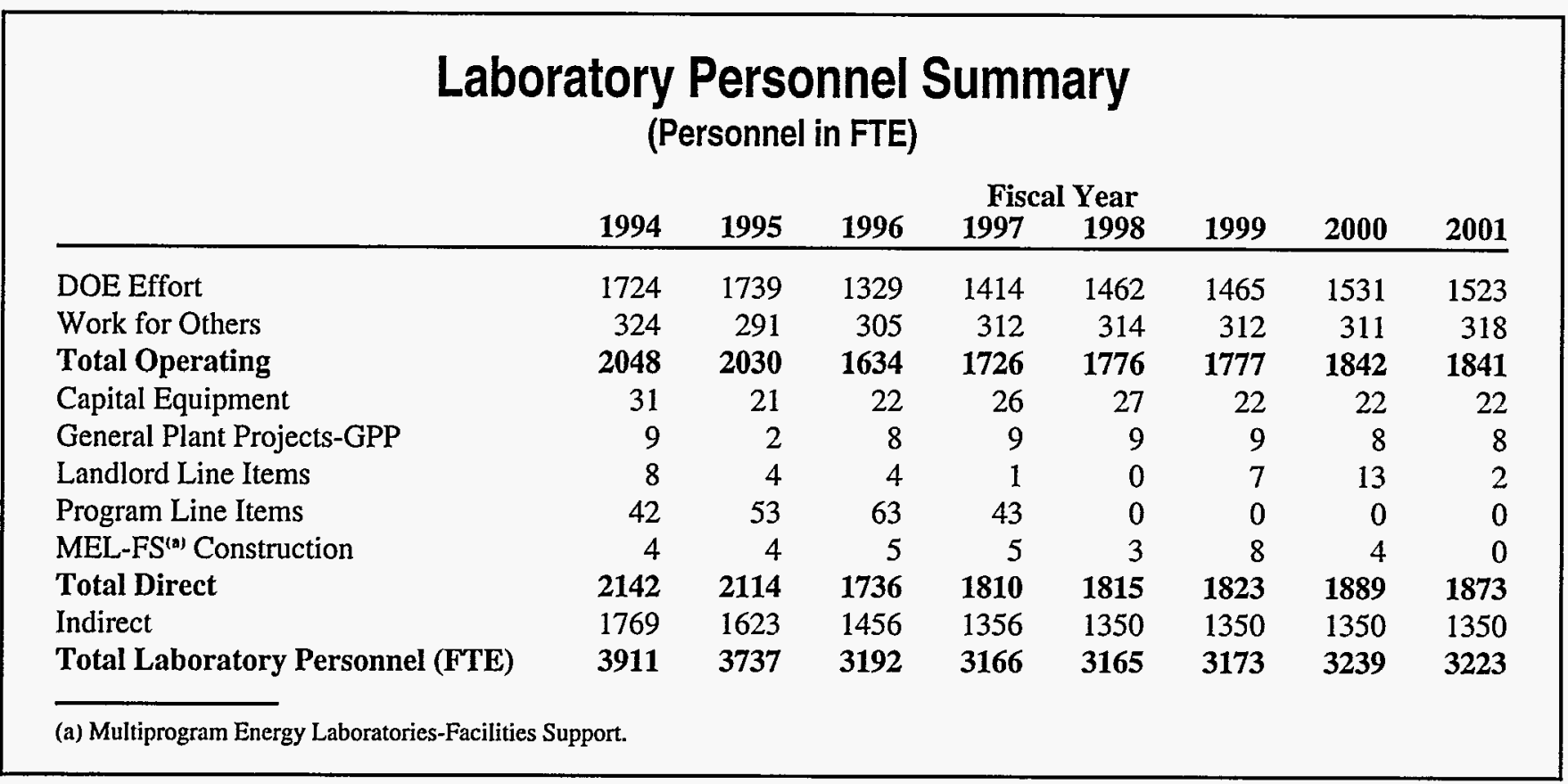




\section{Funding by Secretarial Officer}

(Budget Authorization \$ in Millions)(a) $^{(a)}$

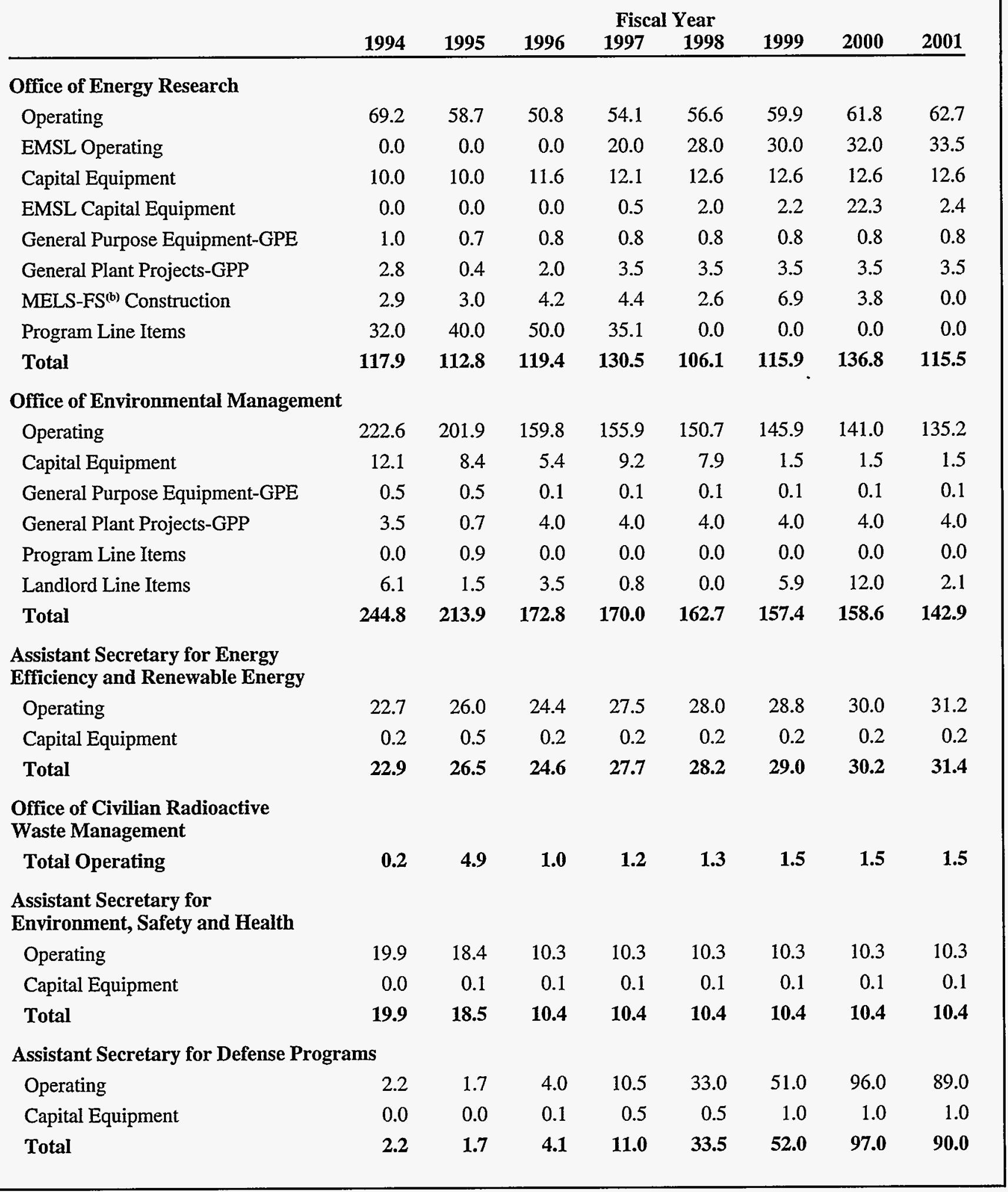




\title{
Funding by Secretarial Officer (contd)
}

\author{
(Budget Authorization \$ in Millions) $)^{(a)}$
}

\begin{tabular}{llllllll}
\multicolumn{8}{c}{ Fiscal Year } \\
1994 & 1995 & 1996 & 1997 & 1998 & 1999 & 2000 & 2001 \\
\hline
\end{tabular}

Office of Science Education

\& Technical Information

Total Operating

Office of Nonproliferation and

National Security

Operating

Capital Equipment

Line Item

Total

Assistant Secretary for

Nuclear Energy

Total Operating

Assistant Secretary for

Fossil Energy

Total Operating

Office of Policy

Total Operating

Other DOE Organizations

TA Nat'l Energy Information System BPA

Total Operating

Other DOE Sites

Total Operating

Total DOE Programs

Operating

Capital Equipment

General Purpose Equipment-GPE

General Plant Projects-GPP

Landlord Line Items

Program Line Items

MEL-FS ${ }^{(b)}$ Construction

Total
1.7

$1.7 \quad 1.3$

1.0

1.0

1.0

1.0

1.0

1.0

$\begin{array}{rrrrrrrr}30.2 & 29.5 & 27.0 & 27.0 & 27.0 & 27.3 & 27.3 & 27.3 \\ 1.3 & 1.1 & 1.2 & 1.2 & 1.2 & 1.2 & 1.2 & 1.2 \\ 0.0 & 0.0 & 0.0 & 0.0 & 0.0 & 0.0 & 0.0 & 0.0 \\ \mathbf{3 1 . 5} & \mathbf{3 0 . 6} & \mathbf{2 8 . 2} & \mathbf{2 8 . 2} & \mathbf{2 8 . 2} & \mathbf{2 8 . 5} & \mathbf{2 8 . 5} & \mathbf{2 8 . 5}\end{array}$

$\begin{array}{llllllll}0.6 & 74.4 & 41.5 & 50.5 & 50.5 & 50.5 & 50.5 & 51.5\end{array}$

\begin{tabular}{l|l|l|l|l|l|l|l}
1.7 & 1.9 & 1.8 & 2.6 & 3.4 & 3.4 & 3.4 & 3.4
\end{tabular}

$\begin{array}{llllllll}1.4 & 0.7 & 1.2 & 1.2 & 1.2 & 1.2 & 1.2 & 1.2\end{array}$

$\begin{array}{llllllll}0.1 & 0.2 & 0.0 & 0.0 & 0.0 & 0.0 & 0.0 & 0.0\end{array}$

$\begin{array}{llllllll}1.8 & 1.0 & 1.5 & 1.5 & 1.5 & 1.5 & 1.5 & 1.5\end{array}$

$\begin{array}{llllllll}1.9 & 1.2 & 1.5 & 1.5 & 1.5 & 1.5 & 1.5 & 1.5\end{array}$

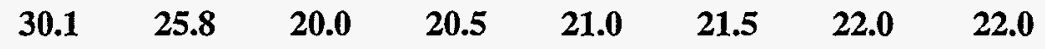

$\begin{array}{llllllll}404.4 & 446.4 & 344.3 & 383.8 & 413.5 & 433.8 & 479.5 & 471.3\end{array}$

$\begin{array}{llllllll}23.6 & 20.1 & 18.6 & 23.8 & 24.5 & 18.8 & 38.9 & 19.0\end{array}$

$\begin{array}{llllllll}1.5 & 1.2 & 0.9 & 0.9 & 0.9 & 0.9 & 0.9 & 0.9\end{array}$

$\begin{array}{llllllll}6.3 & 1.1 & 6.0 & 7.5 & 7.5 & 7.5 & 7.5 & 7.5\end{array}$

$\begin{array}{llllllll}6.1 & 2.5 & 3.5 & 0.8 & 0.0 & 5.9 & 12.0 & 2.1\end{array}$

$\begin{array}{llllllll}32.0 & 40.9 & 50.0 & 35.1 & 0.0 & 0.0 & 0.0 & 0.0\end{array}$

$\begin{array}{lllllllll}2.9 & 3.0 & 4.2 & 4.4 & 2.6 & 6.9 & 3.8 & 0.0\end{array}$

$\begin{array}{llllllll}476.8 & 515.2 & 427.5 & 456.3 & 449.0 & 473.8 & 542.6 & 500.8\end{array}$ 


\title{
Funding by Secretarial Officer (contd)
}

\author{
(Budget Authorization \$ in Millions) $)^{(a)}$
}

\begin{tabular}{lrrrrrrrr} 
& & \multicolumn{7}{c}{ Fiscal Year } \\
& $\mathbf{1 9 9 4}$ & $\mathbf{1 9 9 5}$ & $\mathbf{1 9 9 6}$ & $\mathbf{1 9 9 7}$ & $\mathbf{1 9 9 8}$ & $\mathbf{1 9 9 9}$ & $\mathbf{2 0 0 0}$ & $\mathbf{2 0 0 1}$ \\
\hline Work for Others & & & & & & & & \\
Nuclear Regulatory Commission & 14.8 & 10.9 & 11.0 & 11.0 & 11.0 & 11.0 & 11.0 & 11.0 \\
Department of Defense & 50.0 & 32.7 & 51.0 & 55.0 & 58.0 & 60.0 & 62.0 & 64.0 \\
Environmental Protection Agency & 3.3 & 2.7 & 2.0 & 2.0 & 2.0 & 2.0 & 2.0 & 2.0 \\
NASA and Other Federal Agencies & 6.8 & 12.8 & 10.0 & 10.0 & 10.0 & 10.0 & 10.0 & 10.0 \\
Other - Nonfederal Agencies & 0.4 & 0.3 & 1.0 & 1.0 & 1.0 & 1.0 & 1.0 & 1.0 \\
Total & $\mathbf{7 5 . 3}$ & $\mathbf{5 9 . 4}$ & $\mathbf{7 5 . 0}$ & $\mathbf{7 9 . 0}$ & $\mathbf{8 2 . 0}$ & $\mathbf{8 4 . 0}$ & $\mathbf{8 6 . 0}$ & $\mathbf{8 8 . 0}$ \\
Total Laboratory Funding & & & & & & & & \\
Operating & & & & & & & & \\
Capital Equipment & 479.7 & 505.8 & 419.3 & 462.8 & 495.5 & 517.8 & 565.5 & 559.3 \\
General Purpose Equipment-GPE & 23.6 & 20.1 & 18.6 & 23.8 & 24.5 & 18.8 & 38.9 & 19.0 \\
General Plant Projects-GPP & 1.5 & 1.2 & 0.9 & 0.9 & 0.9 & 0.9 & 0.9 & 0.9 \\
Landlord Line Items & 6.3 & 1.1 & 6.0 & 7.5 & 7.5 & 7.5 & 7.5 & 7.5 \\
Program Line Items & 6.1 & 2.5 & $\mathbf{3 . 5}$ & 0.8 & 0.0 & 5.9 & 12.0 & 2.1 \\
MEL-FS (b) Construction & 32.0 & 40.9 & 50.0 & 35.1 & 0.0 & 0.0 & 0.0 & 0.0 \\
Total & 2.9 & 3.0 & 4.2 & 4.4 & 2.6 & 6.9 & 3.8 & 0.0 \\
& $\mathbf{5 5 2 . 1}$ & $\mathbf{5 7 4 . 6}$ & $\mathbf{5 0 2 . 5}$ & $\mathbf{5 3 5 . 3}$ & $\mathbf{5 3 1 . 0}$ & $\mathbf{5 5 7 . 8}$ & $\mathbf{6 2 8 . 6}$ & $\mathbf{5 8 8 . 8}$
\end{tabular}

(a) Budget authorization escalated through FY 2001.

(b) Multiprogram Energy Laboratories-Facilities Support. 


\section{Direct Personnel by Secretarial Officer \\ (Personnel in FTE)}

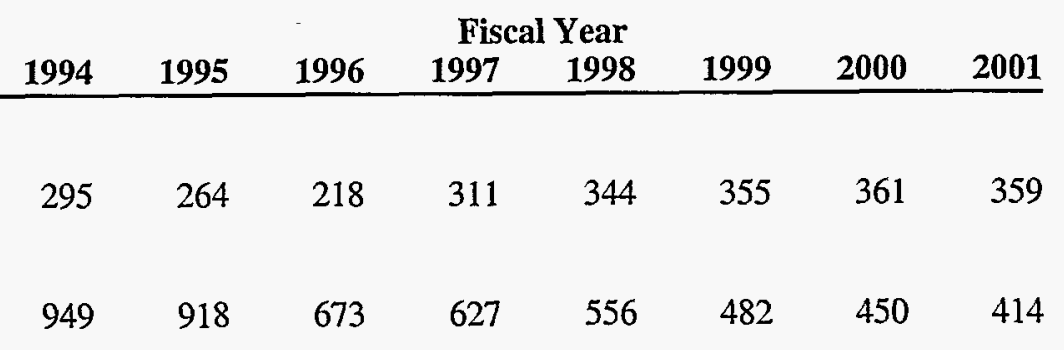

Assistant Secretary for Energy

Efficiency and Renewable Energy

Operating

Office of Civilian Radioactive

Waste Management

Operating

$\begin{array}{llllllll}1 & 22 & 4 & 5 & 5 & 6 & 6 & 6\end{array}$

Assistant Secretary for

Environment, Safety and Health

Operating

$\begin{array}{llllllll}85 & 84 & 45 & 44 & 42 & 41 & 40 & 39\end{array}$

Assistant Secretary for

Defense Programs

Operating

$\begin{array}{llllllll}9 & 8 & 17 & 45 & 135 & 203 & 300 & 335\end{array}$

Office of Science Education

\& Technical Information

Operating

$\begin{array}{lllllllll}7 & 6 & 4 & 4 & 4 & 4 & 4 & 4\end{array}$

Office of Nonproliferation

and National Security

Operating

$\begin{array}{llllllll}129 & 134 & 117 & 114 & 111 & 109 & 106 & 103\end{array}$

Assistant Secretary for

Nuclear Energy

Operating

$\begin{array}{lll}3 & 51 \quad 38\end{array}$

39

39

39

39

39

Assistant Secretary for

Fossil Energy

Operating

79

8

11

14

14

13

13

Office of Policy

Operating

63

5

$\begin{array}{lllll}5 & 5 & 5 & 5 & 5\end{array}$




\section{Direct Personnel by Secretarial Officer (contd)}

(Personnel in FTE)

\begin{tabular}{llllllll}
\multicolumn{8}{c}{ Fiscal Year } \\
1994 & 1995 & 1996 & 1997 & 1998 & 1999 & 2000 & 2001 \\
\hline
\end{tabular}

Other DOE Organizations

Operating

Other DOE Sites

Operating

Total DOE Program

Work for Others

Nuclear Regulatory Commission

Department of Defense

Environmental Protection Agency

NASA and Other Federal Agencies

Other - Nonfederal Agencies

Total

Laboratory Direct

Operating

Capital Equipment

General Plant Projects-GPP

Landlord Line Items

Program Line Items

MEL-FS ${ }^{(a)}$ Construction

Total Laboratory Direct

Total Laboratory Indirect

Total Laboratory Personnel (FTE)
$8 \quad 5$

$$
7
$$

6

6

6

6

$\begin{array}{rrrrrrrr}128 & 117 & 87 & 87 & 86 & 86 & 85 & 83 \\ \mathbf{1 7 2 4} & \mathbf{1 7 3 9} & \mathbf{1 3 2 9} & \mathbf{1 4 1 4} & \mathbf{1 4 6 2} & \mathbf{1 4 6 5} & \mathbf{1 5 3 1} & \mathbf{1 5 2 3}\end{array}$

$\begin{array}{rrrrrrrr}64 & 46 & 45 & 43 & 42 & 41 & 40 & 40 \\ 215 & 179 & 207 & 217 & 222 & 223 & 224 & 231 \\ 14 & 11 & 8 & 8 & 8 & 7 & 7 & 7 \\ 29 & 54 & 41 & 40 & 38 & 37 & 36 & 36 \\ 2 & 1 & 4 & 4 & 4 & 4 & 4 & 4 \\ \mathbf{3 2 4} & \mathbf{2 9 1} & \mathbf{3 0 5} & \mathbf{3 1 2} & \mathbf{3 1 4} & \mathbf{3 1 2} & \mathbf{3 1 1} & \mathbf{3 1 8}\end{array}$

2048

$$
2048
$$

$31 \quad 21 \quad 22$

921

$8 \quad 4$

$42 \quad 53 \quad 63$

44

$2142 \quad 2114 \quad 1736$

$\begin{array}{lll}1769 & 1623 & 1456\end{array}$

3911

$\begin{array}{rrrrr}1726 & 1776 & 1777 & 1842 & 1841 \\ 26 & 27 & 22 & 22 & 22 \\ 9 & 9 & 9 & 8 & 8 \\ 1 & 0 & 7 & 13 & 2 \\ 43 & 0 & 0 & 0 & 0 \\ 5 & 3 & 8 & 4 & 0\end{array}$

$\begin{array}{lllll}1810 & 1815 & 1823 & 1889 & 1873\end{array}$

$\begin{array}{lllll}1356 & 1350 & 1350 & 1350 & 1350\end{array}$

$\begin{array}{lllll}3166 & 3165 & 3173 & 3239 & 3223\end{array}$

(a) Multiprogram Energy Laboratories-Facilities Support. 


\section{Resources by Major DOE Areas}

(Budget Authorization \$ in Millions)(a)

\begin{tabular}{|c|c|c|c|c|c|c|c|c|}
\hline & & & & & Fiscal & & 2000 & \\
\hline & 1994 & 1990 & 1990 & 1991 & 1998 & 1999 & 2000 & 2001 \\
\hline Office of Energy Research & & & & & & & & \\
\hline Magnetic Fusion (AT) & 3.4 & 3.5 & 3.4 & 3.4 & 3.4 & 3.4 & 3.4 & 3.4 \\
\hline High Energy Physics (KA) & 0.2 & 0.0 & 0.1 & 0.1 & 0.1 & 0.1 & 0.1 & 0.1 \\
\hline Nuclear Physics (KB) & 0.3 & 0.0 & 0.0 & 0.0 & 0.0 & 0.0 & 0.0 & 0.0 \\
\hline Basic Energy Sciences (KC) & 11.3 & 11.4 & 12.1 & 14.0 & 14.8 & 15.6 & 16.5 & 17.4 \\
\hline Biological/Environ Research Prog Dir (KR) & 0.0 & 0.0 & 0.0 & 0.0 & 0.0 & 0.0 & 0.0 & 0.0 \\
\hline Advisory \& Oversight Program Dir. (KF) & 0.0 & 0.0 & 0.0 & 0.0 & 0.0 & 0.0 & 0.0 & 0.0 \\
\hline ER Laboratory Technology Transfer (KU) & 16.0 & 7.9 & 3.2 & 3.6 & 4.3 & 4.8 & 4.8 & 4.8 \\
\hline Multiprogram Energy Laboratories (KG) & 0.1 & 0.7 & 0.7 & 0.7 & 0.7 & 0.7 & 0.7 & 0.7 \\
\hline Energy Research Analysis (KD) & 0.4 & 0.5 & 0.3 & 0.3 & 0.3 & 0.3 & 0.3 & 0.3 \\
\hline Biological \& Environmental Research (KP) & 37.5 & 34.7 & 31.0 & 32.0 & 33.0 & 35.0 & 36.0 & 36.0 \\
\hline EMSL Operations (KP - BER) & 0.0 & 0.0 & 0.0 & 20.0 & 28.0 & 30.0 & 32.0 & 33.5 \\
\hline Operating & 69.2 & 58.7 & 50.8 & 74.1 & 84.6 & 89.9 & 93.8 & 96.2 \\
\hline Capital Equipment (AT) & 0.2 & 0.2 & 0.1 & 0.1 & 0.1 & 0.1 & 0.1 & 0.1 \\
\hline Capital Equipment (KC) & 1.1 & 1.0 & 1.5 & 2.0 & 2.5 & 2.5 & 2.5 & 2.5 \\
\hline Capital Equipment (KP) & 8.7 & 8.8 & 10.0 & 10.0 & 10.0 & $\cdot 10.0$ & 10.0 & 10.0 \\
\hline EMSL Capital (KP - BER) & 0.0 & 0.0 & 0.0 & 0.5 & 2.1 & 2.2 & 22.3 & 2.4 \\
\hline Capital Equipment & 10.0 & 10.0 & 11.6 & 12.6 & 14.7 & 14.8 & 34.9 & 15.0 \\
\hline General Purpose Equipment-GPE (KP) & 1.0 & 0.7 & 0.8 & 0.8 & 0.8 & 0.8 & 0.8 & 0.8 \\
\hline General Plant Projects-GPP (KP) & 2.8 & 0.4 & 2.0 & 3.5 & 3.5 & 3.5 & 3.5 & 3.5 \\
\hline Multiprogram Energy Laboratories (KG) & 2.9 & 3.0 & 4.2 & 4.4 & 2.6 & 6.9 & 3.8 & 0.0 \\
\hline Program Line Item (EMSL) & 32.0 & 40.0 & 50.0 & 35.1 & 0.0 & 0.0 & 0.0 & 0.0 \\
\hline Total Funding & 117.9 & 112.8 & 119.4 & 130.5 & 106.2 & 115.9 & 136.8 & 115.5 \\
\hline Direct Personnel & & & & & & & & \\
\hline Operating & 295 & 264 & 218 & 311 & 344 & 355 & 361 & 359 \\
\hline Capital & 13 & 13 & 15 & 16 & 19 & 19 & 19 & 19 \\
\hline General Plant Projects-GPP & 4 & 1 & 3 & 4 & 4 & 4 & 4 & 4 \\
\hline Multiprogram Energy Laboratories (KG) & 4 & 4 & 5 & 5 & 3 & 8 & 4 & 0 \\
\hline Program Line Items & 42 & 52 & 63 & 43 & 0 & 0 & 0 & 0 \\
\hline Total Direct Personnel & 358 & 334 & 304 & 379 & 370 & 386 & 388 & 382 \\
\hline
\end{tabular}




\section{Resources by Major DOE Areas (contd)}

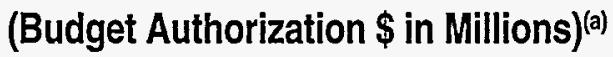

\begin{tabular}{lrrrrrrrr} 
& & & \multicolumn{7}{c}{ Fiscal Year } \\
& $\mathbf{1 9 9 4}$ & $\mathbf{1 9 9 5}$ & $\mathbf{1 9 9 6}$ & $\mathbf{1 9 9 7}$ & $\mathbf{1 9 9 8}$ & $\mathbf{1 9 9 9}$ & $\mathbf{2 0 0 0}$ & $\mathbf{2 0 0 1}$ \\
\hline Office of Environmental Management & & & & & & & & \\
Technical Development (EW) & 28.4 & 24.8 & 28.0 & 33.0 & 38.0 & 44.0 & 44.0 & 39.0 \\
Waste Management (EW) & 160.6 & 142.9 & 108.0 & 105.0 & 98.0 & 87.0 & 80.0 & 81.0 \\
Waste Management (EX) & 10.0 & 19.0 & 12.3 & 10.0 & 7.4 & 7.5 & 9.6 & 7.7 \\
Environmental Restoration (EW) & 23.3 & 15.2 & 11.5 & 7.9 & 7.3 & 7.4 & 7.4 & 7.5 \\
Environmental Restoration (EX) & 0.3 & 0.0 & 0.0 & 0.0 & 0.0 & 0.0 & 0.0 & 0.0 \\
Total Operating & $\mathbf{2 2 2 . 6}$ & $\mathbf{2 0 1 . 9}$ & $\mathbf{1 5 9 . 8}$ & $\mathbf{1 5 5 . 9}$ & $\mathbf{1 5 0 . 7}$ & $\mathbf{1 4 5 . 9}$ & $\mathbf{1 4 1 . 0}$ & $\mathbf{1 3 5 . 2}$ \\
Capital Equipment (EW) & 12.1 & 7.4 & $\mathbf{5 . 4}$ & 9.2 & 7.9 & 1.5 & 1.5 & 1.5 \\
General Purpose Equipment-GPE & 0.5 & 0.5 & 0.1 & 0.1 & 0.1 & 0.1 & 0.1 & 0.1 \\
General Plant Projects-GPP & 3.5 & 0.7 & 4.0 & 4.0 & 4.0 & 4.0 & 4.0 & 4.0 \\
Program Line Items & 0.0 & 0.9 & 0.0 & 0.0 & 0.0 & 0.0 & 0.0 & 0.0 \\
Landlord Line Items & 6.1 & 2.5 & 3.5 & 0.8 & 0.0 & 5.9 & 12.0 & 2.1 \\
Total Funding & $\mathbf{2 4 4 . 8}$ & $\mathbf{2 1 3 . 9}$ & $\mathbf{1 7 2 . 8}$ & $\mathbf{1 7 0 . 0}$ & $\mathbf{1 6 2 . 7}$ & $\mathbf{1 5 7 . 4}$ & $\mathbf{1 5 8 . 6}$ & $\mathbf{1 4 2 . 9}$ \\
Direct Personnel & & & & & & & & \\
Operating & & & & & & & \\
Capital Equipment & 949 & 918 & 673 & 627 & 556 & 482 & 450 & 414 \\
General Plant Projects-GPP & $\mathbf{1 6}$ & 7 & $\mathbf{5}$ & 8 & 7 & 2 & 2 & 2 \\
Program Line Items & $\mathbf{5}$ & 1 & $\mathbf{5}$ & $\mathbf{5}$ & $\mathbf{5}$ & $\mathbf{5}$ & 4 & 4 \\
Landlord Line Items & 0 & 214 & 173 & 170 & 163 & 157 & 159 & 143 \\
Total Direct Personnel & 6 & 3 & 4 & 1 & 0 & 6 & 12 & 2 \\
& $\mathbf{9 7 6}$ & $\mathbf{1 1 4 3}$ & $\mathbf{8 6 0}$ & $\mathbf{8 1 1}$ & $\mathbf{7 3 1}$ & $\mathbf{6 5 2}$ & $\mathbf{6 2 7}$ & $\mathbf{5 6 5}$ \\
& & & & & & & & \\
\hline & & & & & & &
\end{tabular}




\section{Resources by Major DOE Areas (contd)}

(Budget Authorization $\$$ in Millions)(a) $^{(\text {) }}$

\begin{tabular}{|c|c|c|c|c|c|c|c|c|}
\hline & \multirow[b]{2}{*}{1994} & \multirow[b]{2}{*}{1995} & \multirow[b]{2}{*}{1996} & \multirow[b]{2}{*}{1997} & \multicolumn{2}{|c|}{ Fiscal Year } & \multirow[b]{2}{*}{2000} & \multirow[b]{2}{*}{2001} \\
\hline & & & & & 1998 & 1999 & & \\
\hline \multicolumn{9}{|l|}{ Efficiency and Renewable Energy } \\
\hline Electric Energy Systems (AK) & 2.3 & 1.6 & 2.0 & 2.3 & 2.7 & 2.7 & 2.7 & 2.7 \\
\hline Electric Energy Systems (AL) & 1.1 & 0.1 & 0.8 & 0.8 & 0.8 & 0.8 & 0.8 & 0.8 \\
\hline Geothermal Systems (AM) & 0.1 & 0.0 & 0.0 & 0.0 & 0.0 & 0.0 & 0.0 & 0.0 \\
\hline Hydrogen Research (AR) & 0.2 & 0.1 & 0.0 & 0.0 & 0.0 & 0.0 & 0.0 & 0.0 \\
\hline Solar Energy (EB) & 1.3 & 0.8 & 0.0 & 0.0 & 0.0 & 0.0 & 0.0 & 0.0 \\
\hline Buildings Sector-EERE (EC) & 11.6 & 15.7 & 11.0 & 13.2 & 12.6 & 12.6 & 13.1 & 13.6 \\
\hline Industrial Energy Conservation (ED) & 3.6 & 4.2 & 4.7 & 4.8 & 5.0 & 5.2 & 5.4 & 5.6 \\
\hline Transportation Sector (EE) & 1.0 & 1.5 & 2.5 & 3.0 & 3.5 & 4.0 & 4.5 & 5.0 \\
\hline Tech \& Financial Assistance-EERE (EF) & 0.3 & 1.0 & 1.1 & 1.1 & 1.1 & 1.2 & 1.2 & 1.2 \\
\hline Utility Sector (EK) & 0.3 & 0.3 & 0.3 & 0.3 & 0.3 & 0.3 & 0.3 & 0.3 \\
\hline Policy And Management (EH) & 0.2 & 0.4 & 1.0 & 1.0 & 1.0 & 1.0 & 1.0 & 1.0 \\
\hline Policy And Management (KK04) & 0.7 & 0.3 & 1.0 & 1.0 & 1.0 & 1.0 & 1.0 & 1.0 \\
\hline Operating & 22.7 & 26.0 & 24.4 & 27.5 & 28.0 & 28.8 & 30.0 & 31.2 \\
\hline Capital Equipment & 0.2 & 0.5 & 0.2 & 0.2 & 0.2 & 0.2 & 0.2 & 0.2 \\
\hline Total Funding & 22.9 & 26.5 & 24.6 & 27.7 & 28.2 & 29.0 & 30.2 & 31.4 \\
\hline Direct Operating Personnel & 97 & 118 & 106 & 116 & 115 & 115 & 116 & 117 \\
\hline \multicolumn{9}{|l|}{$\begin{array}{l}\text { Office of Civilian Radioactive } \\
\text { Waste Management }\end{array}$} \\
\hline Nuclear Waste Fund (DB) ${ }^{(b)}$ & -0.2 & 0.3 & 0.5 & 0.7 & 0.8 & 1.0 & 1.0 & 1.0 \\
\hline Civilian Radioactive Waste R\&D (DC) & 0.4 & 4.6 & 0.5 & 0.5 & 0.5 & 0.5 & 0.5 & 0.5 \\
\hline Total Funding & 0.2 & 4.9 & 1.0 & 1.2 & 1.3 & 1.5 & 1.5 & 1.5 \\
\hline Direct Operating Personnel & 1 & 22 & 4 & 5 & 5 & 6 & 6 & 6 \\
\hline \multicolumn{9}{|l|}{$\begin{array}{l}\text { Assistant Secretary for Environment, } \\
\text { Safety and Health }\end{array}$} \\
\hline Environmental, Safety and Health (HA-01) & 16.1 & 13.1 & 6.1 & 6.1 & 6.1 & 6.1 & 6.1 & 6.1 \\
\hline Program Direction (HA-04) & 0.0 & 0.8 & 0.5 & 0.5 & 0.5 & 0.5 & 0.5 & 0.5 \\
\hline Office of Security Evaluation (HS) & 2.9 & 1.6 & 2.0 & 2.0 & 2.0 & 2.0 & 2.0 & 2.0 \\
\hline Nuclear Safety Oversight (NS) & 0.7 & 2.0 & 1.5 & 1.5 & 1.5 & 1.5 & 1.5 & 1.5 \\
\hline Radiological Oversight (NR) & 0.2 & 0.1 & 0.2 & 0.2 & 0.2 & 0.2 & 0.2 & 0.2 \\
\hline Operating & 19.9 & 17.6 & 10.3 & $\mathbf{1 0 . 3}$ & 10.3 & 10.3 & 10.3 & 10.3 \\
\hline Capital Equipment (HA-01) & 0.0 & 0.1 & 0.1 & 0.1 & 0.1 & 0.1 & 0.1 & 0.1 \\
\hline Capital Equipment (HS) & 0.0 & 0.0 & 0.0 & 0.0 & 0.0 & 0.0 & 0.0 & 0.0 \\
\hline Total Funding & 19.9 & 17.7 & 10.4 & 10.4 & 10.4 & 10.4 & 10.4 & 10.4 \\
\hline Direct Operating Personnel & 85 & 84 & 45 & 44 & 42 & 41 & 40 & 39 \\
\hline
\end{tabular}




\section{Resources by Major DOE Areas (contd)}

(Budget Authorization \$ in Millions)(a) $^{()^{2}}$

\begin{tabular}{|c|c|c|c|c|c|c|c|c|}
\hline & \multirow[b]{2}{*}{1994} & \multirow{2}{*}{1995} & \multirow{2}{*}{1996} & \multirow{2}{*}{1997} & \multicolumn{2}{|c|}{ Fiscal Year } & \multirow{2}{*}{2000} & \multirow{2}{*}{2001} \\
\hline & & & & & 1998 & 1999 & & \\
\hline \multicolumn{9}{|l|}{ Assistant Secretary for Defense Programs } \\
\hline Materials Production (GE) & 2.2 & 1.7 & 4.0 & 10.5 & 33.0 & 51.0 & 96.0 & 89.0 \\
\hline Operating & 2.2 & 1.7 & 4.0 & 10.5 & 33.0 & 51.0 & 96.0 & 89.0 \\
\hline Capital Equipment & 0.0 & 0.0 & 0.1 & 0.5 & 0.5 & 1.0 & 1.0 & 1.0 \\
\hline Total Funding & 2.2 & 1.7 & 4.1 & 11.0 & 33.5 & $\mathbf{5 2 . 0}$ & 97.0 & $\mathbf{9 0 . 0}$ \\
\hline Direct Operating Personnel & 9 & 8 & 17 & 45 & 135 & 203 & 300 & 335 \\
\hline \multicolumn{9}{|l|}{$\begin{array}{l}\text { Office of Science Education } \\
\& \text { Technical Information }\end{array}$} \\
\hline University \& Science Education (KT) & 1.7 & 1.3 & 1.0 & 1.0 & 1.0 & 1.0 & 1.0 & 1.0 \\
\hline Univ. \& Science Education - Defense (KV) & 0.0 & 0.0 & 0.0 & 0.0 & 0.0 & 0.0 & 0.0 & 0.0 \\
\hline Total Funding & 1.7 & 1.3 & 1.0 & 1.0 & 1.0 & 1.0 & 1.0 & 1.0 \\
\hline Direct Operating Personnel & 7 & 6 & 4 & 4 & 4 & 4 & 4 & 4 \\
\hline \multicolumn{9}{|l|}{$\begin{array}{l}\text { Office of Nonproliferation and } \\
\text { National Security }\end{array}$} \\
\hline Emergency Managemnt Division (GB0506) & 1.6 & 0.2 & 0.1 & 0.1 & 0.1 & 0.1 & 0.1 & 0.1 \\
\hline Verification \& Control Technology (GC) & 17.3 & 15.2 & 14.5 & 14.5 & 14.5 & 14.8 & 14.8 & 14.8 \\
\hline Nuclear Safeguards \& Security (GD) & 3.8 & 2.6 & 3.0 & 3.0 & 3.0 & 3.0 & 3.0 & 3.0 \\
\hline Expt Control, Nonprolif \& Safeguards (GJ) & 2.7 & 6.2 & 4.8 & 4.8 & 4.8 & 4.8 & 4.8 & 4.8 \\
\hline Intelligence (NT) & 4.8 & 5.3 & 4.6 & 4.6 & 4.6 & 4.6 & 4.6 & 4.6 \\
\hline Operating & 30.2 & 29.5 & 27.0 & 27.0 & 27.0 & 27.3 & 27.3 & 27.3 \\
\hline Capital Equipment (GC) & 1.3 & 1.1 & 1.2 & 1.2 & 1.2 & 1.2 & 1.2 & 1.2 \\
\hline Capital Equipment (GD) & 0.0 & 0.0 & 0.0 & 0.0 & 0.0 & 0.0 & 0.0 & 0.0 \\
\hline Capital Equipment (NT) & 0.0 & 0.0 & 0.0 & 0.0 & 0.0 & 0.0 & 0.0 & 0.0 \\
\hline Total Funding & 31.5 & 30.6 & 28.2 & 28.2 & 28.2 & 28.5 & 28.5 & 28.5 \\
\hline \multicolumn{9}{|l|}{ Direct Personnel } \\
\hline Operating & 129 & 134 & 117 & 114 & 111 & 109 & 106 & 103 \\
\hline Capital Equipment & 2 & 1 & 2 & 2 & 1 & 1 & 1 & 1 \\
\hline Total Direct Personnel & 131 & 135 & 119 & 116 & 112 & 110 & 107 & 104 \\
\hline
\end{tabular}




\section{Resources by Major DOE Areas (contd)}

(Budget Authorization $\$$ in Millions) $^{(a)}$

\begin{tabular}{|c|c|c|c|c|c|c|c|c|}
\hline \multirow{3}{*}{ Assistant Secretary for Nuclear Energy } & \multirow[b]{2}{*}{1994} & \multirow[b]{2}{*}{1995} & \multirow[b]{2}{*}{1996} & \multirow[b]{2}{*}{1997} & \multicolumn{2}{|c|}{ Fiscal Year } & \multirow[b]{2}{*}{2000} & \multirow[b]{2}{*}{2001} \\
\hline & & & & & 1998 & 1999 & & \\
\hline & & & & & & & & \\
\hline Nuclear Energy R\&D (AF) & 0.2 & 71.2 & 41.0 & 50.0 & 50.0 & 50.0 & 50.0 & 51.0 \\
\hline Uranium Enrichment (CD) & 0.0 & 0.1 & 0.0 & 0.0 & 0.0 & 0.0 & 0.0 & 0.0 \\
\hline Policy \& Management (KK) & 0.4 & 2.3 & 0.5 & 0.5 & 0.5 & 0.5 & 0.5 & 0.5 \\
\hline Isotope Production (ST) & 0.0 & 0.8 & 0.0 & 0.0 & 0.0 & 0.0 & 0.0 & 0.0 \\
\hline Total Funding ${ }^{(b)}$ & 0.6 & 74.4 & 41.5 & 50.5 & 50.5 & $\mathbf{5 0 . 5}$ & 50.5 & 51.5 \\
\hline Direct Operating Personnel & 3 & 51 & 38 & 39 & 39 & 39 & 39 & 39 \\
\hline \multicolumn{9}{|l|}{ Assistant Secretary for Fossil Energy } \\
\hline Coal (AA) & 1.3 & 1.0 & 1.2 & 1.2 & 1.2 & 1.2 & 1.2 & 1.2 \\
\hline Petroleum (AC) & 0.1 & 0.5 & 0.6 & 1.4 & 2.2 & 2.2 & 2.2 & 2.2 \\
\hline Total Funding & 1.4 & 1.5 & 1.8 & 2.6 & 3.4 & 3.4 & 3.4 & 3.4 \\
\hline Direct Operating Personnel & 7 & 9 & 8 & 11 & 14 & 14 & 13 & 13 \\
\hline \multicolumn{9}{|l|}{ Office of Policy } \\
\hline Policy, Planning \& Analysis (PE) & 1.4 & 0.7 & 1.2 & 1.2 & 1.2 & 1.2 & 1.2 & 1.2 \\
\hline Total Funding & 1.4 & 0.7 & 1.2 & 1.2 & 1.2 & 1.2 & 1.2 & 1.2 \\
\hline Direct Operating Personnel & 6 & 3 & 5 & 5 & 5 & 5 & 5 & 5 \\
\hline \multicolumn{9}{|l|}{ Other DOE Organizations } \\
\hline Nat'l Energy Information System (TA) & 0.1 & 0.2 & 0.0 & 0.0 & 0.0 & 0.0 & 0.0 & 0.0 \\
\hline Bonneville Power Administration & 1.8 & 1.0 & 1.5 & 1.5 & 1.5 & 1.5 & 1.5 & 1.5 \\
\hline Total Funding & 1.9 & 1.2 & 1.5 & 1.5 & 1.5 & 1.5 & 1.5 & 1.5 \\
\hline Direct Personnel & 8 & 5 & 7 & 6 & 6 & 6 & 6 & 6 \\
\hline \multicolumn{9}{|l|}{ Other DOE Sites } \\
\hline Total Funding & 30.1 & 25.8 & 20.0 & 20.5 & 21.0 & 21.5 & 22.0 & 22.0 \\
\hline Direct Personnel & 128 & 117 & 87 & 87 & 86 & 86 & 85 & 83 \\
\hline \multicolumn{9}{|l|}{ Total DOE Programs } \\
\hline Operating & 404.4 & 446.4 & 344.3 & 383.8 & 413.5 & 433.8 & 479.5 & 471.3 \\
\hline Capital Equipment & 23.6 & 20.1 & 18.6 & 23.8 & 24.5 & 18.8 & 38.9 & 19.0 \\
\hline General Purpose Equipment-GPE & 1.5 & 1.2 & 0.9 & 0.9 & 0.9 & 0.9 & 0.9 & 0.9 \\
\hline General Plant Projects-GPP & 6.3 & 1.1 & 6.0 & 7.5 & 7.5 & 7.5 & 7.5 & 7.5 \\
\hline Landlord Line Items & 6.1 & 2.5 & 3.5 & 0.8 & 0.0 & 5.9 & 12.0 & 2.1 \\
\hline Program Line Items & 32.0 & 40.9 & 50.0 & 35.1 & 0.0 & 0.0 & 0.0 & 0.0 \\
\hline MEL-FS' ${ }^{(c)}$ Construction & 2.9 & 3.0 & 4.2 & 4.4 & 2.6 & 6.9 & 3.8 & 0.0 \\
\hline Total Funding & 476.8 & 515.2 & 427.5 & 456.3 & 449.0 & 473.8 & 542.6 & 500.8 \\
\hline
\end{tabular}




\section{Resources by Major DOE Areas (contd)}

(Budget Authorization \$ in Millions)(a) $^{(\text {) }}$

Fiscal Year

\begin{tabular}{llllllll}
1994 & 1995 & 1996 & 1997 & 1998 & 1999 & 2000 & 2001 \\
\hline
\end{tabular}

Total DOE Programs (Contd)

Direct Personnel

Operating

Capital

General Plant Projects (GPP)

Landlord Line Items

Program Line Items

MEL-FS ${ }^{(c)}$ Construction

Total Direct Personnel

Work for Others

Nuclear Regulatory Commission

Operating

Direct Personnel

Department of Defense

Operating

Direct Personnel

Environmental Protection Agency

Operating

Direct Personnel

NASA and Other Federal Agencies

Operating

Direct Personnel

Other - Nonfederal Agencies

Operating

Direct Personnel

Total Work for Others

Operating

Direct Personnel

$\begin{array}{rrrrrrrr}1724 & 1739 & 1329 & 1414 & 1462 & 1465 & 1531 & 1523 \\ 31 & 21 & 22 & 26 & 27 & 22 & 22 & 22 \\ 9 & 2 & 8 & 9 & 9 & 9 & 8 & 8 \\ 8 & 4 & 4 & 1 & 0 & 7 & 13 & 2 \\ 42 & 53 & 63 & 43 & 0 & 0 & 0 & 0 \\ 4 & 4 & 5 & 5 & 3 & 8 & 4 & 0 \\ 1818 & 1823 & 1431 & 1498 & 1501 & 1511 & 1578 & 1555\end{array}$

$\begin{array}{llllllll}14.8 & 10.9 & 11.0 & 11.0 & 11.0 & 11.0 & 11.0 & 11.0 \\ 64 & 46 & 45 & 43 & 42 & 41 & 40 & 40\end{array}$

$\begin{array}{llllllll}50.0 & 32.7 & 51.0 & 55.0 & 58.0 & 60.0 & 62.0 & 64.0\end{array}$

$\begin{array}{llllllll}215 & 179 & 207 & 217 & 222 & 223 & 224 & 231\end{array}$

$\begin{array}{cccccccc}3.3 & 2.7 & 2.0 & 2.0 & 2.0 & 2.0 & 2.0 & 2.0 \\ 14 & 11 & 8 & 8 & 8 & 7 & 7 & 7\end{array}$

$\begin{array}{llllllll}6.8 & 12.8 & 10.0 & 10.0 & 10.0 & 10.0 & 10.0 & 10.0 \\ 29 & 54 & 41 & 40 & 38 & 37 & 36 & 36\end{array}$

$\begin{array}{llllllll}0.4 & 0.3 & 1.0 & 1.0 & 1.0 & 1.0 & 1.0 & 1.0 \\ 2 & 1 & 4 & 4 & 4 & 4 & 4 & 4\end{array}$

$\begin{array}{llllllll}\mathbf{7 5 . 3} & \mathbf{5 9 . 4} & \mathbf{7 5 . 0} & \mathbf{7 9 . 0} & \mathbf{8 2 . 0} & \mathbf{8 4 . 0} & \mathbf{8 6 . 0} & \mathbf{8 8 . 0}\end{array}$ $\begin{array}{llllllll}324 & 291 & 305 & 312 & 314 & 312 & 311 & 318\end{array}$ 


\section{Resources by Major DOE Areas (contd)}

(Budget Authorization \$ in Millions)(a) $^{(a)}$

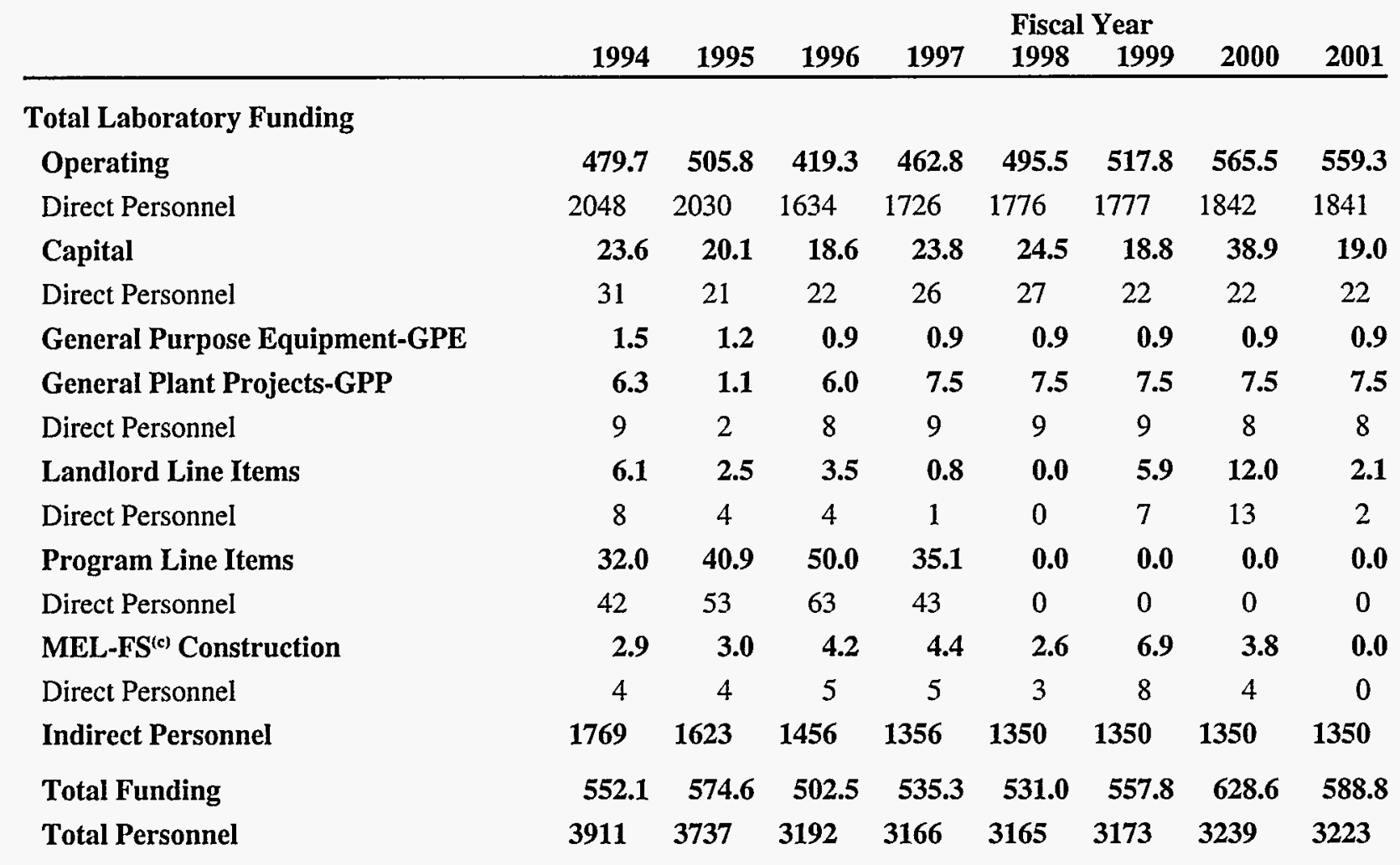

(a) Budget authorization escalated through FY 2001.

(b) Negative balances result from deobligation of prior year uncosted funds.

(c) Multiprogram Energy Laboratories-Facilities Support. 


\section{Subcontracting and Procurement}

The Laboratory is dependent upon external resources (universities and industry) for support in achieving timely and successful completion of assigned programs and projects. This is accomplished by staff in the Contracts organization who use the procurement acquisition process in acquiring needed equipment, materials, supplies, and services. The table below reflects actual subcontracted amounts for FY 1995 and projections for FY 1996 through FY 1998.

\section{Small and Disadvantaged Business Procurement}

The Laboratory is committed to support the socioeconomic objectives of
DOE and has established procedures and programs that support meeting those objectives. The Laboratory received the Secretary of Energy's FY 1994 award for achievement in meeting and exceeding the Laboratory's small business goals.

\section{Subcontracting and Procurement Table}

(Dollar Amounts in Millions)

\begin{tabular}{lrrrr} 
& \multicolumn{5}{c}{ Fiscal Year } \\
& $\mathbf{1 9 9 5}$ & $\mathbf{1 9 9 6}$ & \multicolumn{1}{c}{$\mathbf{1 9 9 7}$} & $\mathbf{1 9 9 8}$ \\
\hline Obligated & 205.5 & 198.7 & 188.3 & 192.6 \\
Subcontracting and Procurement from: & & & & \\
$\quad$ Universities & 5.9 & 5.7 & 5.4 & 5.5 \\
$\quad$ All Others & 190.9 & 184.5 & 174.8 & 178.8 \\
$\quad$ Other DOE & 8.7 & 8.5 & 8.1 & 8.3 \\
Total External Subcontracts and Procurements & $\mathbf{2 0 5 . 5}$ & $\mathbf{1 9 8 . 7}$ & $\mathbf{1 8 8 . 3}$ & $\mathbf{1 9 2 . 6}$
\end{tabular}

\section{Small and Disadvantaged Business Procurement Table}

(Dollar Amounts in Millions)

\begin{tabular}{lrrrr} 
& \multicolumn{4}{c}{ Fiscal Year } \\
\hline Procurement from Small Business & 132.5 & $63.1 \%$ & 101.9 & $51.7 \%$ \\
Procurement from Disadvantaged Businesses & 21.2 & $10.7 \%$ & 13.7 & $6.9 \%$
\end{tabular}




\section{Pacific Northwest National Laboratory}

\section{Managed and Operated by Battelle for the U.S. Department of Energy}

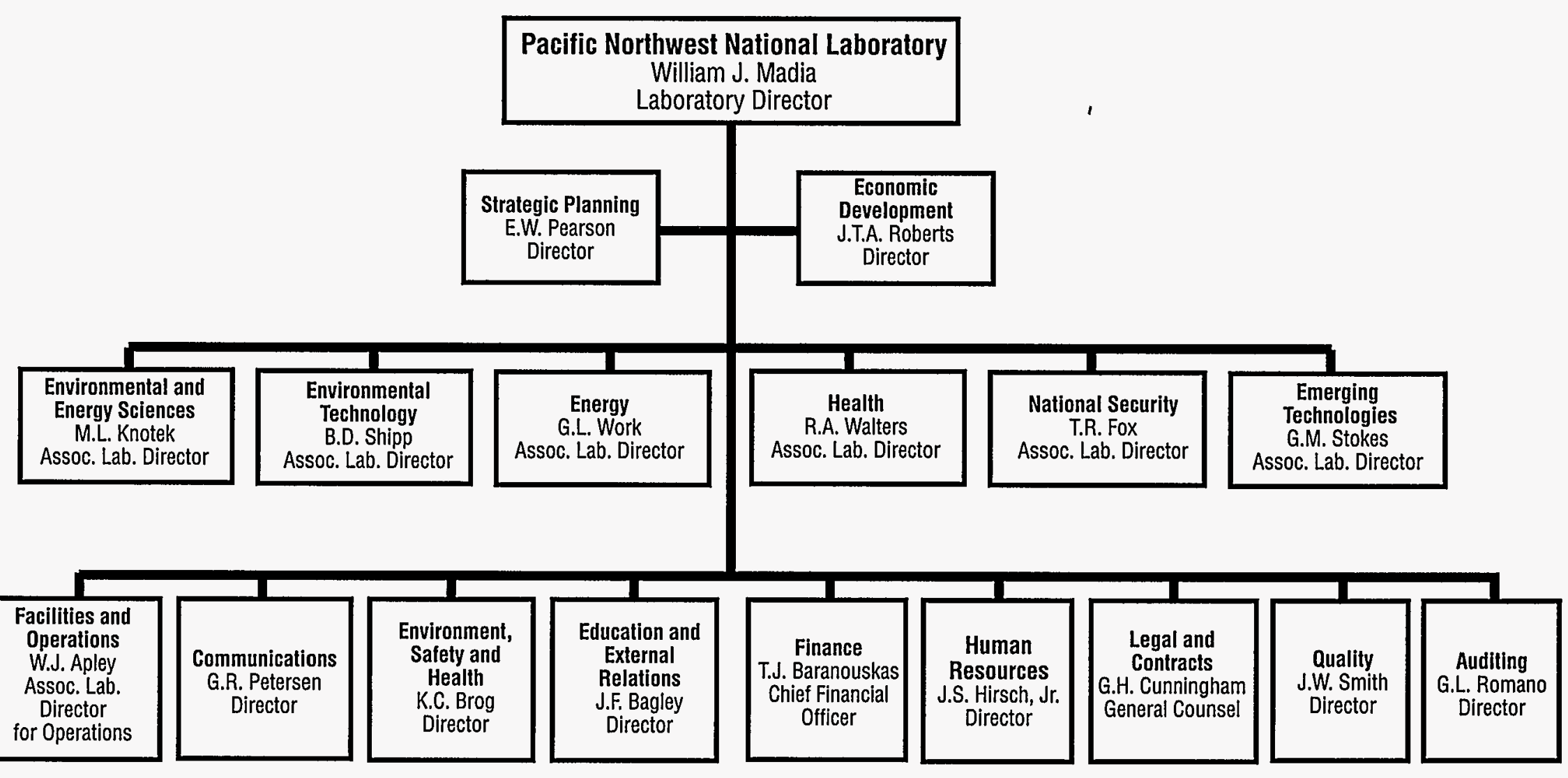




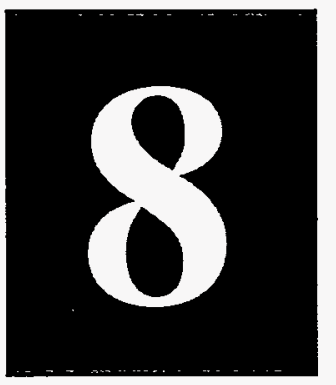

\section{Acronyms and Abbreviations}

AEDOT

AISES

ALARA

AMTEX

ANSI

APEL

ARM

ASCOT

ASME

ATPO

AVLIS

AWU-NW

BES

BESP

CART

CATS

CEDR

CERCLA

CHAMMP

CINDY

CP/MAS

CRADA

CSEPP

DAMA

DHHS

DNA

DOD

DOE

DO-IT

EEO/AA

EFRA

EM

EMSL

EPA

EPAct

EPIC

EPRI

ER

ER-LTT

ES\&H

ESEECAP

ESI-MS

ESNET

ESP
Advanced Energy Design and Operation Technologies

American Indian Science and Engineering Society

as low as reasonably achievable

American Textile Industry

American National Standards Institute

Advanced Process Engineering Laboratory

Atmospheric Radiation Measurement

Atmospheric Studies in Complex Terrain

American Society of Mechanical Engineers

Applied Technology Program Office

Advanced Vapor Laser Isotope Separation

Associated Western Universities Inc., Northwest Division

Office of Basic Energy Sciences

Building Energy Standards Program

Cloud and Radiation Testbed

Corrective Action Tracking System

Comprehensive Epidemiologic Data Resource

Comprehensive Environmental Response, Compensation, and Liability Act

Computer Hardware, Advanced Mathematics and Model Physics

Code for Internal Dosimetry

cross polarization/magic-angle spinning

Cooperative Research and Development Agreement

Chemical Stockpile Emergency Preparedness Program

Demand Activated Manufacturing Architecture

Department of Health and Human Services

deoxyribonucleic acid

U.S. Department of Defense

U.S. Department of Energy

Disabilities, Opportunities, Internetworking and Technology

Equal Employment Opportunity/Affirmative Action

Electronic Forms, Routing, and Authorization

Office of Environmental Management

Environmental Molecular Sciences Laboratory

U.S. Environmental Protection Agency

Energy Policy Act

Energy Pollution Prevention Information Clearing House

Electric Power Research Institute

Office of Energy Research

Office of Energy Research Laboratory Technology Transfer Program

Environment, Safety, and Health

Environmental Science and Engineering Education Coordination and Assistance Project

electrospray ionization mass spectrometry

Energy Sciences NETwork

Environmental Sustainability Project 


\begin{tabular}{|c|c|}
\hline FDDI & fiber digital data interface \\
\hline $\mathrm{FE}$ & Office of Fossil Energy \\
\hline FEDS & Federal Energy Decision System \\
\hline FEMA & Federal Emergency Management Agency \\
\hline FEMIS & Federal Emergency Management Information System \\
\hline FEMP & Federal Energy Management Program \\
\hline FFCA & Federal Facility Compliance Act \\
\hline FFTF & Fast Flux Test Facility \\
\hline FLC & Federal Laboratory Consortium \\
\hline FTE & full-time equivalent \\
\hline FTICR & Fourier transform ion cyclotron resonance \\
\hline FT-ICRMS & Fourier transform ion cyclotron resonance mass spectrometry \\
\hline FY & fiscal year \\
\hline GCAM & Global Change Assessment Model \\
\hline GChM & Global Chemistry Model \\
\hline GCMs & general circulation models \\
\hline GEC & Global Environmental Change \\
\hline GEM & Graduate Degrees for Minorities in Engineering and Science \\
\hline GPE & General Purpose Equipment \\
\hline GPF & General Purpose Facilities \\
\hline GPP & General Plant Projects \\
\hline $\mathrm{HBCU}$ & Historically Black Colleges and Universities \\
\hline HEIS & Hanford Environmental Information System \\
\hline HEPA & high-efficiency particulate air \\
\hline HESEC & Hanford Environmental Science and Engineering Consortium \\
\hline HTIP & Hanford Technology Integration Program \\
\hline HVAC & heating, ventilation, and air-conditioning \\
\hline IAEA & International Atomic Energy Agency \\
\hline IEEE & Institute of Electrical and Electronics Engineers \\
\hline IGEX & International Germanium Experiment \\
\hline IGBP & International Geosphere Biosphere Program \\
\hline INEL & Idaho National Engineering Laboratory \\
\hline IPCC & Intergovernmental Panel on Climate Change \\
\hline ISFIT & ion sensitive field-effect transistor \\
\hline ITER & International Thermonuclear Experimental Reactor \\
\hline ITTS & Integrated Technology Transfer System \\
\hline LAN & Local Area Network \\
\hline LANL & Los Alamos National Laboratory \\
\hline LBL & Lawrence Berkeley Laboratory \\
\hline LDRD & Laboratory Directed Research and Development \\
\hline LLNL & Lawrence Livermore National Laboratory \\
\hline MAP & Manufactured Housing Acquisition Program \\
\hline MEL-FS & Multiprogram Energy Laboratories-Facilities Support \\
\hline MEPAS & Multimedia Environmental Pollutant Assessment System \\
\hline MESA & Mathematics, Engineering and Science Achievement \\
\hline MOU & Memorandum of Understanding \\
\hline MSCF & Molecular Science Computing Facility \\
\hline NASA & National Aeronautics and Space Administration \\
\hline NE & Office of Nuclear Energy \\
\hline NEPA & National Environmental Policy Act \\
\hline NERSC & National Energy Research Supercomputing Center \\
\hline NFPA & National Fire Protection Association \\
\hline NIEHS & National Institute of Environmental Health Sciences \\
\hline
\end{tabular}




\begin{tabular}{|c|c|}
\hline NIH & National Institutes of Health \\
\hline NIS/IPP & New Independent States Industrial Partnering Program \\
\hline NMSS & Office of Nuclear Material Safety and Safeguards \\
\hline NOBChE & National Organization of Black Chemical Engineers \\
\hline NPAR & Nuclear Plant Aging Research \\
\hline NRC & Nuclear Regulatory Commission \\
\hline NREL & National Renewable Energy Laboratory \\
\hline NRR & Nuclear Reactor Regulation \\
\hline NSBE & National Society for Black Engineers \\
\hline OFCCP & Office of Federal Contract Compliance Programs \\
\hline OGCMs & ocean general circulation models \\
\hline OHER & Office of Health and Environmental Research \\
\hline ORNL & Oak Ridge National Laboratory \\
\hline OSHA & Occupational Safety and Health Administration \\
\hline OSPI & Office of the Superintendent of Public Instruction \\
\hline P2 & Pollution Prevention \\
\hline PASS & Pacific Northwest National Laboratory Affiliate Staff Scientist \\
\hline & personal computer \\
\hline PG\&E & Pacific Gas and Electric \\
\hline $\mathrm{PO}$ & Policy Office \\
\hline RAAS & Remedial Action Assessment System \\
\hline RadCon & Radiological Control \\
\hline RAISE & Research Internships for Students with Disabilities \\
\hline RCRA & Resource Conservation and Recovery Act \\
\hline RES & Office of Nuclear Regulatory Research \\
\hline RI/FS & remedial investigation/feasibility study \\
\hline RLWS & Radioactive Liquid Waste System \\
\hline RPIS2 & Real Property Inventory System \\
\hline RW & Office of Civilian Radioactive Waste Management \\
\hline SARs & Safety Analysis Reports \\
\hline SDEP & Staff Diversity Enhancement Program \\
\hline SERS & Science and Engineering Research Semester \\
\hline SHPE & Society of Hispanic Professional Engineers \\
\hline SISR & Surface/Interface Structure Reactions \\
\hline SMES & superconducting magnetic energy storage \\
\hline SNFP & Spent Nuclear Fuel Project \\
\hline SQL & Standard Query Language \\
\hline SSP & Subsurface Science Program \\
\hline STCG & Site Technology Coordination Group \\
\hline SWE & Society of Women Engineers \\
\hline SWTS & Solid Waste Technical Support \\
\hline TCP/IP & Transmission Control Protocol/Internet Protocol \\
\hline TDPO & Technology Development Program Office \\
\hline TEES & Thermochemical Environment Energy System \\
\hline TRAC & Teacher Research Associates \\
\hline $\mathrm{TReC}$ & Textile Resource Conservation Project \\
\hline TWINS & Tank Waste Information Network System \\
\hline TWRS & Tank Waste Remediation System \\
\hline USCAR & U.S. Council for Automotive Research \\
\hline USEC & U.S. Enrichment Corporation \\
\hline VPP & Voluntary Protection Program \\
\hline
\end{tabular}


WDOE Washington Department of Ecology

WIPP Waste Isolation Pilot Plant

WHC

WM

Westinghouse Hanford Company

WSU-TC

Waste Management and Technology Program

Washington State University at Tri-Cities

XAS $\quad \mathrm{x}$-ray absorption spectroscopy 
